\title{
Topological Groups: \\ Yesterday, Today, Tomorrow
}


Sidney A. Morris (Ed.)

\section{Topological Groups: Yesterday, Today, Tomorrow}


This book is a reprint of the Special Issue that appeared in the online, open access journal, Axioms (ISSN 2075-1680) from 2015-2016, available at:

http://www.mdpi.com/journal/axioms/special_issues/topological_groups_yesterd ay_today_tomorrow

Guest Editor

Sidney A. Morris

School of Science, IT, and Engineering

Federation University Australia

Australia

Editorial Office

MDPI AG

St. Alban-Anlage 66

Basel, Switzerland

Publisher

Shu-Kun Lin

Managing Editor

Qiang Liu

\section{Edition 2016}

MDPI • Basel • Beijing $\bullet$ Wuhan $\bullet$ Barcelona $\bullet$ Belgrade

ISBN 978-3-03842-268-6 (Hbk)

ISBN 978-3-03842-269-3 (electronic)

Articles in this volume are Open Access and distributed under the Creative Commons Attribution license (CC BY), which allows users to download, copy and build upon published articles even for commercial purposes, as long as the author and publisher are properly credited, which ensures maximum dissemination and a wider impact of our publications. The book taken as a whole is ( 2016 MDPI, Basel, Switzerland, distributed under the terms and conditions of the Creative Commons by Attribution (CC BY-NC-ND) license (http://creativecommons.org/licenses/by-nc-nd/4.0/). 


\section{Table of Contents}

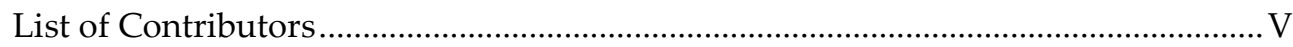

About the Guest Editor............................................................................................. VII

Sidney A. Morris

An Overview of Topological Groups: Yesterday, Today, Tomorrow

Reprinted from: Axioms 2016, 5(2), 11

http://www.mdpi.com/2075-1680/5/2/11 ...............................................................

Karl H. Hofmann and Sidney A. Morris

Pro-Lie Groups: A Survey with Open Problems

Reprinted from: Axioms 2015, 4(3), 294-312

http://www.mdpi.com/2075-1680/4/3/294

\section{W. W. Comfort and Dieter Remus}

Non-Abelian Pseudocompact Groups

Reprinted from: Axioms 2016, 5(1), 2

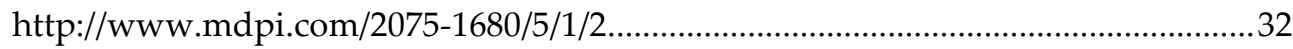

\section{Morris W. Hirsch}

Fixed Points of Local Actions of Lie Groups on Real and Complex 2-Manifolds

Reprinted from: Axioms 2015, 4(3), 313-320

http://www.mdpi.com/2075-1680/4/3/313. .56

\section{Ol'ga Sipacheva}

Free Boolean Topological Groups

Reprinted from: Axioms 2015, 4(4), 492-517

http://www.mdpi.com/2075-1680/4/4/492 


\section{Mikhail Tkachenko}

Lindelöf $\Sigma$-Spaces and R-Factorizable Paratopological Groups

Reprinted from: Axioms 2015, 4(3), 254-267

http://www.mdpi.com/2075-1680/4/3/254.

\section{Saak Gabriyelyan}

On T-Characterized Subgroups of Compact Abelian Groups

Reprinted from: Axioms 2015, 4(2), 194-212

http://www.mdpi.com/2075-1680/4/2/194.....

\section{Dikran Dikranjan, Anna Giordano Bruno and Daniele Impieri}

Characterized Subgroups of Topological Abelian Groups

Reprinted from: Axioms 2015, 4(4), 459-491

http://www.mdpi.com/2075-1680/4/4/459.

\section{Lydia Außenhofer, Dikran Dikranjan and Elena Martín-Peinador}

Locally Quasi-Convex Compatible Topologies on a Topological Group

Reprinted from: Axioms 2015, 4(4), 436-458

http://www.mdpi.com/2075-1680/4/4/436

\section{Viorel Nitica and Andrei Török}

Open and Dense Topological Transitivity of Extensions by Non-Compact Fiber of Hyperbolic Systems: A Review

Reprinted from: Axioms 2015, 4(1), 84-101

http://www.mdpi.com/2075-1680/4/1/84. 


\section{List of Contributors}

Lydia Außenhofer Faculty of Computer Science and Mathematics, Universität Passau, Innstr. 33, Passau D-94032, Germany.

W. W. Comfort Department of Mathematics, Wesleyan University, Middletown, CT 06457, USA.

Dikran Dikranjan Dipartimento di Matematica e Informatica, Università degli Studi di Udine, Via delle Scienze 206, Udine 33100, Italy.

Saak Gabriyelyan Department of Mathematics, Ben-Gurion University of the Negev, P.O. 653, Beer-Sheva 8410501, Israel.

Anna Giordano Bruno Dipartimento di Matematica e Informatica, Università degli Studi di Udine, Via delle Scienze 206, Udine 33100, Italy.

Morris W. Hirsch Department of Mathematics, University of California, Berkeley, CA 94720-3840, USA; Department of Mathematics, University of Wisconsin, Madison, WI 53706-1388, USA.

Karl H. Hofmann Department of Mathematics, Tulane University, New Orleans, LA 70118, USA; Fachbereich Mathematik, Technische Universität Darmstadt, Schlossgartenstrasse 7, Darmstadt 64289, Germany.

Daniele Impieri Dipartimento di Matematica e Informatica, Università degli Studi di Udine, Via delle Scienze 206, Udine 33100, Italy.

Elena Martín-Peinador Instituto de Matemática Interdisciplinar y Departamento de Geometría y Topología, Universidad Complutense de Madrid, Madrid 28040, Spain.

Sidney A. Morris Department of Mathematics and Statistics, School of Engineering and Mathematical Sciences, La Trobe University, Bundoora, Victoria 3086, Australia; Faculty of Science and Technology, Federation University Australia, Victoria 3353, Australia.

Viorel Nitica Institute of Mathematics of the Romanian Academy, P.O. Box 1-764, RO-70700 Bucharest, Romania; Department of Mathematics, West Chester University, West Chester, PA 19383, USA.

Dieter Remus Institut für Mathematik, Universität Paderborn, Warburger Str. 100, Paderborn D-33095, Germany.

Ol'ga Sipacheva Department of General Topology and Geometry, Lomonosov Moscow State University, Leninskie Gory 1, Moscow 119991, Russia. 
Mikhail Tkachenko Universidad Autónoma Metropolitana, Iztapalapa Campus, Av. San Rafael Atlixco 187, Col. Vicentina, C.P. 09340 Iztapalapa, Mexico City, Mexico.

Andrei Török Department of Mathematics, University of Houston, 651 PGH, Houston, TX 77204-3008, USA; Institute of Mathematics of the Romanian Academy, P.O. Box 1-764, RO-70700 Bucharest, Romania. 


\section{About the Guest Editor}

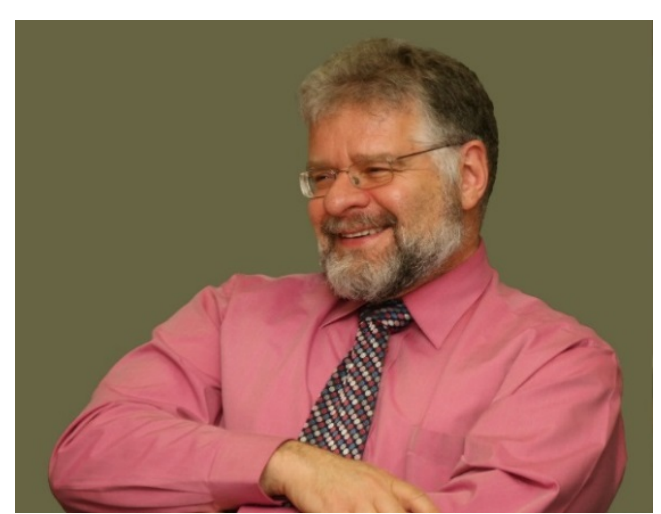

Sidney Morris, Emeritus Professor of Federation University, Australia and Adjunct Professor of La Trobe University, obtained his $\mathrm{BSc}$ (Hons) from the University of Queensland in 1969 and his PhD from Flinders University in 1970. He has been (full) Professor, Department Head, Dean, Vice-President, CAO and CEO. He was employed by nine Australian universities and universities in Israel, the UK, and USA. He was the Editor of the Bulletin of the Australian Mathematical Society and the Journal of Research and Practice in Information Technology, a founding Editor of the Journal of Group Theory, and the Australian Mathematical Lecture Series. He is an Editor of the Gazette of the Australian Mathematical Society and on the Editorial Board of Axioms. He received the Lester R. Ford Award from the Mathematical Association of America. He has published 160 journal papers mostly on topological group theory, and four books, plus an online book which has been translated into eight languages. 



\title{
Preface to "An Overview of Topological Groups: Yesterday, Today, Tomorrow"
}

\author{
Sidney A. Morris
}

Reprinted from Axioms. Cite as: Morris, S.A. An Overview of Topological Groups: Yesterday, Today, Tomorrow. Axioms 2016, 5, 11.

It was in 1969 that I began my graduate studies on topological group theory and I often dived into one of the following five books. My favourite book "Abstract Harmonic Analysis" [1] by Ed Hewitt and Ken Ross contains both a proof of the Pontryagin-van Kampen Duality Theorem for locally compact abelian groups and the structure theory of locally compact abelian groups. Walter Rudin's book "Fourier Analysis on Groups" [2] includes an elegant proof of the Pontryagin-van Kampen Duality Theorem. Much gentler than these is "Introduction to Topological Groups" [3] by Taqdir Husain which has an introduction to topological group theory, Haar measure, the Peter-Weyl Theorem and Duality Theory.

Of course the book "Topological Groups" [4] by Lev Semyonovich Pontryagin himself was a tour de force for its time. P. S. Aleksandrov, V.G. Boltyanskii, R.V. Gamkrelidze and E.F. Mishchenko described this book in glowing terms: "This book belongs to that rare category of mathematical works that can truly be called classical - books which retain their significance for decades and exert a formative influence on the scientific outlook of whole generations of mathematicians".

The final book I mention from my graduate studies days is "Topological Transformation Groups" [5] by Deane Montgomery and Leo Zippin which contains a solution of Hilbert's fifth problem as well as a structure theory for locally compact non-abelian groups. These five books gave me a good feeling for the most significant research on locally compact group theory in the first 60 years of the twentieth century. My own contribution to understanding the structure of locally compact abelian groups was a small book "Pontryagin Duality and the Structure of Locally Compact Abelian Groups" [6] which was translated into Russian and served to introduce a generation of young Soviet mathematicians to this topic.

Far from locally compact groups, A.A. Markov [7,8] introduced the study of free topological groups. This was followed up by M.I. Graev in 1948 [9] with a slightly more general concept. Free topological groups are an analogue of free groups in abstract group theory. Markov gave a very long construction of the free topological group on a Tychonoff space and also proved its uniqueness. Graev's proof is also long. Shorter proofs appeared after a few years. Today one derives the existence of Markov and Graev free topological groups from the Adjoint Functor Theorem. Free topological groups have been an active area of research to this day, especially by 
Alexander Vladimirovich Arhangel'skii of Moscow State University and his former doctoral students and they have produced a wealth of deep and interesting results.

Now let me turn to this volume. My aim for "Topological Groups: Yesterday, Today, Tomorrow" is for these articles to describe significant topics in topological group theory in the 20th century and the early 21st century as well as providing some guidance to the future directions topological group theory might take by including some interesting open questions.

"In 1900 David Hilbert presented a seminal address to the International Congress of Mathematicians in Paris. In this address, he initiated a program by formulating 23 problems, which influenced a vast amount of research of the 20th century. The fifth of these problems asked whether every locally-Euclidean topological group admits a Lie group structure. This motivated an enormous volume of work on locally-compact groups during the first half of the 20th century. It culminated in the work of Gleason, Iwasawa, Montgomery, Yamabe and Zippin, yielding a positive answer to Hilbert's fifth problem and exposing the structure of almost connected locally-compact groups [5]. (Recall that a topological group $G$ is called almost connected [10] if the quotient group $G / G_{0}$, modulo the connected component $G_{0}$ of the identity, is compact. The class of almost connected groups includes all compact groups and all connected locally-compact groups.). The advances in the second half of the 20th century shed much light on the structure and representation theory of locally compact groups" is how Karl Heinrich Hofmann and Sidney A. Morris began their article Pro-Lie Groups: A Survey with Open Problems in this volume.

While the class of locally compact abelian groups has the beautiful Pontryagin-van Kampen Duality from which the structure of locally compact abelian groups can be described (see [6]), the structure theory of compact groups has not been derived from any of the various Duality Theorems for compact groups. This led Hofmann and Morris to establish and use a Lie Theory for compact groups to provide a complete description of the structure of compact groups in [11]. They then used in [10] the same Lie Theory approach to establish the structure theory of (almost) connected locally compact groups. As the class of locally compact groups is not closed even under infinite products, they introduced the class of pro-Lie Groups which is a natural extension of the classes of finite-dimensional Lie groups, locally compact abelian groups, compact groups and connected locally compact groups and used the Lie Theory to describe completely the structure of almost connected pro-Lie groups. Their article Pro-Lie Groups: A Survey with Open Problems provides an up-to-date summary of pro-Lie groups and lists 12 interesting questions. Probably the most interesting of these is

Question 2. Let $G$ be a pro-Lie group with identity component $G_{0}$. Is $G / G_{0}$ complete (and therefore, prodiscrete)? 
Over the last 50 years there has been a steady development of the theory of pseudocompact topological groups. In their article Non-abelian Pseudocompact Groups in this volume Wis Comfort and Dieter Remus survey the historical development of the theory of pseudocompact topological groups. They report that "Many of the results we cite, especially the older results, require an abelian hypothesis; some questions, definitions and results make sense and are correct without that hypothesis, however, and we emphasize these. Thus, this paper has two goals: (1) to provide an overview of the (by now substantial) literature on pseudocompact groups; and (2) to offer several new results about non-abelian pseudocompact groups."

In particular Comfort and Remus examine "three recently-established theorems from the literature:

(A) (2006) Every non-metrizable compact abelian group $K$ has $2^{|K|}$-many proper dense pseudocompact subgroups.

(B) (2003) Every non-metrizable compact abelian group $K$ admits $2^{2^{|K|}}$-many strictly finer pseudocompact topological group refinements.

(C) (2007) Every non-metrizable pseudocompact abelian group has a proper dense pseudocompact subgroup and a strictly finer pseudocompact topological group refinement.

(Theorems (A), (B) and (C) become false if the non-metrizable hypothesis is omitted.)". The authors ask: What happens to (A), (B), (C) and to similar known facts about pseudocompact abelian groups if the abelian hypothesis is omitted? Are the resulting statements true, false, true under certain natural additional hypotheses, etc.? Several new results responding in part to these questions are given, and several specific additional questions are posed. One conjecture they mention is due to Comfort and van Mill.

Conjecture 5.4.1. Let $G$ be an abelian group which admits a pseudocompact group topology. Then the supremum of the pseudocompact group topologies on $G$ coincides with the largest totally bounded group topology on $G$ (that is, the topology induced on $G$ by $\operatorname{Hom}(G, \mathbb{T})$.

We mention two of the questions they ask:

Problem 5.7.2. Does every infinite compact group $K$ have $2^{|K|}$-many non-measurable subgroups (of cardinality $|K|$ )?

Problem 8.2.11. * Let $(K, \mathcal{T})$ be a profinite group of uncountable weight.

(a) Does $\mathcal{T}$ admit a proper pseudocompact refinement of maximal weight $2^{|K|}$ ?

(b) Are there $2^{2^{|K|}}$-many pseudocompact group topologies on $K$ which are finer than $\mathcal{T}$ ? 
The next paper we discuss here is Free Boolean Topological Groups by Ol'ga Sipacheva. She introduces her paper as follows: "In the very early 1940s, A. A. Markov $[7,8]$ introduced the free topological group $F(X)$ and the free Abelian topological group $A(X)$ on an arbitrary completely regular Hausdorff topological space $X$ as a topological-algebraic counterpart of the abstract free and free Abelian groups on a set; he also proved the existence and uniqueness of these groups. During the next decade, Graev [9,12], Nakayama [13], and Kakutani [14] simplified the proofs of the main statements of Markov's theory of free topological groups, generalized Markov's construction, and proved a number of important theorems on free topological groups. In particular, Graev generalized the notions of the free and the free Abelian topological group on a space $X$ by identifying the identity element of the free group with an (arbitrary) point of $X$ (the free topological group on $X$ in the sense of Markov coincides with Graev's group on X plus an isolated point), described the topology of free topological groups on compact spaces, and extended any continuous pseudometric on $X$ to a continuous invariant pseudometric on $F(X)$ (and on $A(X)$ ) which is maximal among all such extensions [9].

This study stimulated Mal'tsev, who believed that the most appropriate place of the theory of abstract free groups was in the framework of the general theory of algebraic systems, to introduce general free topological algebraic systems. In 1957, he published the large paper [15], where the basics of the theory of free topological universal algebras were presented.

Yet another decade later, Morris initiated the study of free topological groups in the most general aspect. Namely, he introduced the notion of a variety of topological groups (A definition of a variety of topological groups (determined by a so-called varietal free topological group) was also proposed in 1951 by Higman [16]; however, it is Morris' definition which has proved viable and developed into a rich theory.) and a full variety of topological groups and studied free objects of these varieties [17-19] (see also [20]). Varieties of topological groups and their free objects were also considered by Porst [21], Comfort and van Mill [22], Kopperman, Mislove, Morris, Nickolas, Pestov, and Svetlichny [23], and other authors. Special mention should be made of Dikranjan and Tkachenko's detailed study of varieties of Abelian topological groups with properties related to compactness [24].

The varieties of topological groups in which free objects have been studied best are, naturally, the varieties of general and Abelian topological groups; free and free Abelian precompact groups have also been considered (see, e.g., [25]). However, there is yet another natural variety-Boolean topological groups. Free objects in this variety and its subvarieties have been investigated much less extensively, although they arise fairly often in various studies (especially in the set-theoretic context). The author is aware of only two published papers considering free Boolean topological 
groups from a general point of view: [26], where the topology of the free Boolean topological group on a compact metric space was explicitly described, and [27], where the free Boolean topological groups on compact initial segments of ordinals were classified (see also [28]). The purpose of this paper is to draw attention to these very interesting groups and give a general impression of them. We collect some (known and new) results on free Boolean topological groups, which describe both properties which these groups share with free or free Abelian topological groups and properties specific of free Boolean groups.

We mention here Theorem 8: If $\operatorname{dim} X=0$, then $\operatorname{ind} B(X)=0$, which can be proved much more easily than the analogous result for free topological groups. By contrast, Proposition 9 says: The free Abelian topological group on any connected space has infinitely many connected components, however the free Boolean topological group on any connected space has two connected components. We record here a few of Sipacheva's questions:

Problem 3. Does there exist a space $X$ such that $B(X)$ is normal, but $X^{2}$ is not? Problem 4. Describe spaces $X$ for which $B(X)$ is Lindelöf. Does there exist a space $X$ such that $B(X)$ is Lindelöf, but $X$ is not?

Problem 5. Does there exist a space $X$ for which $B(X)$ is normal (Lindelöf, ccc), but $F(X)$ or $A(X)$ is not?

Problem 6. Is it true that $B(X)$ is Weil complete for any Dieudonné complete space $X$ ?

Problem 7. Is it true that the free (free Boolean) topological group of any stratifiable space is stratifiable?

The article On T-Characterized Subgroups of Compact Abelian Groups by Saak Gabriyelyan addresses $T$-sequences in compact abelian groups. A sequence $\left\{u_{n}\right\}$ in an Abelian group $G$ is called a $T$-sequence if there is a Hausdorff group topology on $G$ relative to which $\lim _{n} u_{n}=0$. A subgroup $H$ of an infinite compact Abelian group $X$ is said to be $T$-characterized if there is a $T$-sequence $\mathbf{u}=\left\{u_{n}\right\}$ in the dual group of $X$ such that $H=\left\{x \in X:\left(u_{n}, x\right) \rightarrow 1\right\}$. The author summarizes the results in this paper as follows: "We show that a closed subgroup $H$ of $X$ is $T$-characterized if and only if $H$ is a $G_{\delta}$-subgroup of $X$ and the annihilator of $H$ admits a Hausdorff minimally almost periodic group topology. All closed subgroups of an infinite compact Abelian group $X$ are $T$-characterized if and only if $X$ is metrizable and connected. We prove that every compact Abelian group $X$ of infinite exponent has a $T$-characterized subgroup which is not an $F_{\sigma}$-subgroup of $X$, that gives a negative answer to Problem 3.3 in [29]".

The next paper we introduce is Characterized Subgroups of Topological Abelian Groups by Dikran Dikranjan, Anna Giordano Bruno and Danele Impieri. Historically, characterized subgroups were studied excusively in the case of the circle group 
$\mathbb{T}$ in the context of Diophantine approximation, dynamical systems and ergodic theory, see for example [30]. A subgroup $H$ of an abelian topological group $X$ is said to be characterized by a sequence $\mathbf{v}=\left(v_{n}\right)$ of characters of $X$ if $H=\left\{x \in X: v_{n}(x) \rightarrow 0\right.$ in $\left.\mathbb{T}\right\}$. The authors say "we introduce the relevant class of auto-characterized groups (namely, the groups that are characterized subgroups of themselves by means of a sequence of non-null characters); in the case of locally compact abelian groups, these are proven to be exactly the non-compact ones. As a by-product of our results, we find a complete description of the characterized subgroups of discrete abelian groups". Amongst the questions presented in the paper, we mention:

Question 5. Are the closed $G_{\delta}$-subgroups of a precompact abelian always $\mathrm{N}$-characterized? (This is equivalent to asking if there exists a continuous injection from $X / F$ into $\mathbb{T}^{n}$ for every closed $G_{\delta}$-subgroup $F$ of a precompact abelian group $X$.)

In the paper Fixed Points of Local Actions of Lie Groups on Real and Complex 2-Manifolds, Morris W. Hirsch surveys "old and new results on fixed points of local actions by Lie groups $G$ on real and complex 2-manifolds. The theme is to find conditions guaranteeing that a compact set of fixed points of a 1-parameter subgroup contains a fixed point of G." The classical results of Poincaré (1885) [31], Hopf (1925) [32] and Lefschetz (1937) [33] yield.

Theorem. Every flow on a compact manifold of nonzero Euler characteristic has a fixed point.

The earliest papers I've found on fixed points for actions of other nondiscrete Lie group are those of P. A. Smith [34] (1942) and H. Wang [35], (1952). Then came Borel [36] with

Theorem. If $H$ is a solvable, irreducible algebraic group over an algebraically closed field $\mathbb{K}$, every algebraic action of $H$ on a complete algebraic variety over $\mathbb{K}$ has a fixed point."

In this paper Hirsch, in particular, puts into context the results of Sommese (1973) [37], Lima (1964) [38], Plante (1986) [39], Bonatti (1992 ) [40], Hirsch (2001) [41], Hirsch (2010) [42], Hirsch (2013) [43] and Hirsch (2014) [44].

Next we turn to the survey paper Open and Dense Topological Transitivity of Extensions by Non-compact Fiber of Hyperbolic Systems - a Review by Viorel Nitica and Andrei Török. They summarize their paper as follows: "Currently there is great renewed interest in proving topological transitivity of various classes of continuous dynamical systems. Even though this is one of the most basic dynamical properties that can be investigated, the tools used by various authors are quite diverse and are 
strongly related to the class of dynamical systems under consideration. The goal of this survey article is to present the state of art for the class of Hölder extensions of hyperbolic systems with non-compact connected Lie group fiber. The hyperbolic systems we consider are mostly discrete time. In particular, we address the stability and genericity of topological transitivity in large classes of such transformations. The paper lists several open problems, conjectures and tries to place this topic of research in the general context of hyperbolic and topological dynamics". The Main Conjecture is:

Conjecture 6. Assume that $X$ is a hyperbolic basic set for $f: X \rightarrow X$ and $\Gamma$ is a finite-dimensional connected Lie group. Among the Hölder cocycles $\beta: X \rightarrow X$ with subexponential growth that are not cohomologous to a cocycle with values in a maximal subsemigroup of $\Gamma$ with non-empty interior, there is a Hölder open and dense set for which the extension $f_{\beta}$ is transitive.

The conjecture is proved for various classes of Lie groups. The techniques used so far are quite diverse and seem to depend heavily on the particular properties of the group that appears in the fiber.

The next paper we discuss is Locally Quasi-Convex Compatible Topologies on a Topological group by Lydia Außenhofer, Dikran Dikranjan and Elena Martín-Peinador.

"Varopoulos posed the question of the description of the group topologies on an abelian group $G$ having a given character group $H$, and called them compatible topologies for the duality $(G ; H),[45]$. As the author explains, the question is motivated by Mackey's Theorem, which holds in the framework of locally convex spaces. He treated the question within the class of locally precompact abelian groups. Later on, this problem was set in a bigger generality in [46]; namely, within the class of locally quasi-convex groups. This is a class of abelian topological groups which properly contains the class of locally convex spaces, a fact which makes the attempt to generalize the Mackey-Arens Theorem more natural".

The authors summarize their results as follows: "For a locally quasi-convex topological abelian group $(G, \tau)$ we study the poset $\mathcal{C}(G, \tau)$ of all locally quasi-convex topologies on $G$ that are compatible with $\tau$ (i.e., have the same dual as $(G, \tau)$ ordered by inclusion. Obviously, this poset has always a bottom element, namely the weak topology $\sigma(G, \widehat{G})$. Whether it has also a top element is an open question. We study both quantitative aspects of this poset (its size) and its qualitative aspects, e.g., its chains and anti-chains. Since we are mostly interested in estimates 'from below', our strategy consists in finding appropriate subgroups $H$ of $G$ that are easier to handle and show that $\mathcal{C}(H)$ and $\mathcal{C}(G / H)$ ) are large and embed, as a poset, in $\mathcal{C}(G, \tau)$. Important special results are: (i) If $K$ is a compact subgroup of a locally quasi-convex group $G$, then $\mathcal{C}(G)$ and $\mathcal{C}(G / K)$ are quasi-isomorphic; (ii) If $D$ is a discrete abelian group of infinite rank, then $\mathcal{C}(D)$ is quasi-isomorphic to the poset 
$\mathfrak{F}_{D}$ of filters on $D$. Combining both results, we prove that for a LCA (locally compact abelian) group $G$ with an open subgroup of infinite co-rank (this class includes, among others, all non $\sigma$-compact LCA groups), the poset $\mathcal{C}(G)$ is as big as the underlying topological structure of $(G, \tau)$ (and set theory) allow. For a metrizable connected compact group X the group of null-sequences $G=c_{0}(X)$ with the topology of uniform convergence is studied. We prove that $C(G)$ is quasi-isomorphic to $\mathcal{P}(\mathbb{R})$." Three questions are recorded below:

Question 7.3. Let $G$ be a non-precompact second countable Mackey group. Is it true that $|\mathcal{C}(G)| \geq \mathfrak{c}$.

Problem 7.4. Find sufficient conditions for a metrizable precompact group $G$ to be Mackey (i.e., have $|\mathcal{C}(G)|=1$.)

Conjecture 7.6. [Mackey dichotomy] For a locally compact group $G$, one has either $|\mathcal{C}(G)|=1$ or $|\mathcal{C}(G)| \geq \mathfrak{c}$.

Last, but certainly not least, we mention Lindelöf $\Sigma$-Spaces and $\mathbb{R}$-Factorizable Paratopological Groups by Mikhail Tkachenko. He summarizes the results as follows: "We prove that if a paratopological group $G$ is a continuous image of an arbitrary product of regular Lindelöf $\Sigma$-spaces, then it is $\mathbb{R}$-factorizable and has countable cellularity. If in addition $G$ is regular, then it is totally $\omega$-narrow, and satisfies $\operatorname{cel}_{\omega}(G) \leq \omega$, and the Hewitt-Nachbin completion of $G$ is again an $\mathbb{R}$-factorizable paratopological group". A curious consequence of the above is Corollary 14: The Sorgenfrey line is not a continuous of any product of regular Lindelöf $\Sigma$-spaces. We conclude by mentioning three questions in this paper:

Problem 15. Let a (Hausdorff) paratopological group $G$ be a continuous image of a product of a family of Lindelöf $\Sigma$-spaces. Does $G$ have the Knaster property? Is it $\omega$-narrow?

Problem 17. Let a Hausdorff (regular) paratopological group $G$ be a continuous image of a dense subspace of a product of separable metrizable spaces. Is $G$ perfectly $\kappa$-normal or $\mathbb{R}$-factorizable?

Problem 18. Does every upper quasi-uniformly continuous quasi-pseudometric on an arbitrary product of Lindelöf $\Sigma$-spaces depend at most on countably many coordinates?

In conclusion, the collection of articles in this volume should give the reader an overview of topological group theory as it developed over the last 115 years, as well as the richness of current research. In this Editorial I have listed some of the open questions in these papers which interested me, but the papers themselves contain many more. My hope is that you, the reader, will solve some of these problems and contribute to the future development of topological group theory. 


\section{References}

1. Hewitt, E.; Ross, K.A. Abstract Harmonic Analysis; Springer-Verlag: Heidelberg, Germany, 1963.

2. Rudin, W. Fourier Analysis on Groups; Wiley: New York, NY, USA, 1962.

3. Husain, T. Introduction to Topological Groups; W.B. Saunders Co.: Philadelphia, PA, USA, 1966.

4. Pontryagin, L.S. Topological Groups; Princeton University Press: Princeton, NJ, USA, 1946.

5. Montgomery, D.; Zippin, L. Topological Transformation Groups; Interscience Publishers: New York, NY, USA, 1955.

6. Morris, S.A. Pontryagin Duality and the Structure of Locally Compact Abelian Groups; Cambridge University Press: Cambridge, UK, 1977.

7. Markov, A.A. On free topological groups. Dokl. Akad. Nauk SSSR 1941 31, 299-301.

8. Markov, A.A. On free topological groups. Izv. Akad. Nauk SSSR Ser. Mat. 1945, 9, 3-64.

9. Graev, M.I. Free topological groups. Izv. Akad. Nauk SSSR Ser. Mat. 1948, 12, 279-324.

10. Hofmann, K.H. The Lie Theory of Connected Pro-Lie Groups; European Mathematical Society: Zürich, Switzerland, 2007.

11. Hofmann, K.H.; Morris, S.A. The Structure of Compact Groups, 3rd ed.; Revised and Augmented; Verlag Walter de Gruyter Berlin: Berlin, Germany, 2013.

12. Graev, M.I. The theory of topological groups I. Uspekhi Mat. Nauk 1950, 5, 3-56.

13. Nakayama, T. Note on free topological groups. Proc. Imp. Acad. Tokyo 1953, 19, 471-475.

14. Kakutani, S. Free topological groups and infinite direct product of topological groups. Proc. Imp. Acad. Tokyo 1944, 20, 595-598.

15. Mal'tsev, A.I. Free topological algebras. Izv. Akad. Nauk SSSR Ser. Mat. 1957, 21, 171-198.

16. Higman, G. Unrestricted free products, and varieties of topological groups. J. Lond. Math. Soc. 1952, 27, 73-81.

17. Morris, S.A. Varieties of topological groups. Bull. Aust. Math. Soc. 1969, 1, 145-160.

18. Morris, S.A. Varieties of topological groups II. Bull. Aust. Math. Soc. 1970, 2, 1-13.

19. Morris, S.A. Varieties of topological groups III. Bull. Aust. Math. Soc. 1970, 2, 165-178.

20. Morris, S.A. Varieties of topological groups: A survey. Colloq. Math. 1982, 46, 147-165.

21. Porst, H.E. On the existence and structure of free topological groups. In Category Theory at Work; Herrlich, H., Porst, H.E., Eds.; Heldermann: Berlin, Germany, 1991; pp. 165-176.

22. Comfort, W.W.; van Mill, J. On the existence of free topological groups. Topol. Appl. 1988, 29, 245-265.

23. Kopperman, R.D.; Mislove, M.W.; Morris, S.A.; Nickolas, P.; Pestov, V.; Svetlichny, S. Limit laws for wide varieties of topological groups. Houst. J. Math. 1996, 22, 307-328.

24. Dikranjan, D.; Tkachenko, M. Varieties generated by countably compact Abelian groups. Proc. Am. Math. Soc. 2002, 130, 2487-2496.

25. Arhangel'skii, A.; Tkachenko, M. Topological Groups and Related Structures; Atlantis Press: Amsterdam, The Netherlands, 2008.

26. Genze, L.V. Free Boolean topological groups. Vestn. Tomsk. Gos. Univ. 2006, 290, 11-13.

27. Genze, L.V.; Gul'ko, S.P.; Khmyleva, T.E. Classification of the free Boolean topological groups on ordinals. Vestn. Tomsk. Gos. Univ. Mat. Mekh. 2008, 1, 23-31. 
28. Genze, L.V.; Gul'ko, S.P.; Khmyleva, T.E. Classification of continuous n-valued function spaces and free periodic topological groups for ordinals. Topol. Proc. 2011, 38, 1-15.

29. Dikranjan, D.; Gabriyelyan, S. On characterized subgroups of compact abelian groups. Topol. Appl. 2013, 160, 2427-2442.

30. Gabriyelyan, S.S. Characterizable groups: Some results and open questions. Topol. Appl. 2012, 159, 2378-2391.

31. Poincaré, H. Sur les courbes définies par une équation différentielle. J. Math. Pures Appl. 1885, 1, 167-244.

32. Hopf, H. Vektorfelder in Mannifgfaltigkeiten. Math. Annalen 1925, 95, 340-367.

33. Lefschetz, S. On the fixed point formula. Ann. Math. 1937, 38, 819-822.

34. Smith, P.A. Stationary points of transformation groups. Proc. Natl. Acad. Sci. USA 1942, 28, 293-297.

35. Hsien-Chung, W. A remark on transformation groups leaving fixed an end point. Proc. Am. Math. Soc. 1952, 3, 548-549.

36. Borel, A. Groupes linéaires algebriques. Ann. Math. 1956, 64, 20-80.

37. Sommese, A. Borel's fixed point theorem for Kaehler manifolds and an application. Proc. Am. Math. Soc. 1973, 41, 51-54.

38. Lima, E. Common singularities of commuting vector fields on 2-manifolds. Comment. Math. Helv. 1964 , 39, 97-110.

39. Plante, J. Fixed points of Lie group actions on surfaces. Ergod. Theory Dyn. Syst. 1986, 6, 149-161.

40. Bonatti, C. Champs de vecteurs analytiques commutants, en dimension 3 ou 4: Existence de zéros communs. Bol. Soc. Brasil. Mat. (N.S.) 1992, 22, 215-247.

41. Hirsch, M.; Weinstein, A. Fixed points of analytic actions of supersoluble Lie groups on compact surfaces. Ergod. Theory Dyn. Syst. 2001, 21, 1783-1787.

42. Hirsch, M. Actions of Lie groups and Lie algebras on manifolds. In A Celebration of the Mathematical Legacy of Raoul Bott; Proceedings \& Lecture Notes 50; Kotiuga, P.R., Ed.; Centre de Recherches Mathématiques, Université de Montréal: Montreal, QC, Canada, 2010.

43. Hirsch, M. Zero sets of Lie algebras of analytic vector fields on real and complex 2-manifolds. Ergod. Theory Dyn. Syst. 2013, arXiv:1310.0081.

44. Hirsch, M. Fixed points of local actions of nilpotent Lie groups on surfaces. Ergod. Theory Dyn. Syst. 2014, doi:10.1017/etds.2015.73.

45. Varopoulos, N.T. Studies in harmonic analysis. Proc. Camb. Phil. Soc. 1964, 60, 467-516.

46. Chasco, M.J.; Martìn-Peinador, E.; Tarieladze, V. On Mackey topology for groups. Stud. Math. 1999, 132, 257-284. 


\title{
Pro-Lie Groups: A Survey with Open Problems
}

\author{
Karl H. Hofmann and Sidney A. Morris
}

Abstract: A topological group is called a pro-Lie group if it is isomorphic to a closed subgroup of a product of finite-dimensional real Lie groups. This class of groups is closed under the formation of arbitrary products and closed subgroups and forms a complete category. It includes each finite-dimensional Lie group, each locally-compact group that has a compact quotient group modulo its identity component and, thus, in particular, each compact and each connected locally-compact group; it also includes all locally-compact Abelian groups. This paper provides an overview of the structure theory and the Lie theory of pro-Lie groups, including results more recent than those in the authors' reference book on pro-Lie groups. Significantly, it also includes a review of the recent insight that weakly-complete unital algebras provide a natural habitat for both pro-Lie algebras and pro-Lie groups, indeed for the exponential function that links the two. (A topological vector space is weakly complete if it is isomorphic to a power $\mathbb{R}^{X}$ of an arbitrary set of copies of $\mathbb{R}$. This class of real vector spaces is at the basis of the Lie theory of pro-Lie groups.) The article also lists 12 open questions connected to pro-Lie groups.

Reprinted from Axioms. Cite as: Hofmann, K.H.; Morris, S.A. Pro-Lie Groups: A Survey with Open Problems. Axioms 2016, 4, 294-312.

\section{Introduction}

In 1900, David Hilbert presented a seminal address to the International Congress of Mathematicians in Paris. In this address, he initiated a program by formulating 23 problems, which influenced a vast amount of research of the 20th century. The fifth of these problems asked whether every locally-Euclidean topological group admits a Lie group structure, see [1]. This motivated an enormous volume of work on locally-compact groups during the first half of the 20th century. It culminated in the work of Gleason, Iwasawa, Montgomery, Yamabe and Zippin, yielding a positive answer to Hilbert's fifth problem and exposing the structure of almost connected locally-compact groups. (Recall that a topological group $G$ is called almost connected if the quotient group $G / G_{0}$, modulo the connected component $G_{0}$ of the identity, is compact. The class of almost connected groups includes all compact groups and all connected locally-compact groups.) The advances in the second half of the 20th century shed much light on the structure and representation theory of locally compact groups. 
Notwithstanding this, it was recognized that the class of locally-compact groups is obviously deficient in that it is not even closed under the formation of arbitrary products. It was unclear which class of topological groups would most appropriately extend the class of finite-dimensional Lie groups and avoid this defect.

At the beginning of the 21st century, the authors of this survey introduced the class of pro-Lie groups [2-4]. This class contains all finite-dimensional Lie groups, all compact groups, all locally-compact Abelian groups and all almost connected locally-compact groups. It is characterized as the class of all closed subgroups of arbitrary products of finite-dimensional Lie groups. Obviously, it is closed under the formation of arbitrary products and even the passage to closed subgroups.

This notion of pro-Lie group differs from that used in the late 20th century, where a group $G$ is called a pro-Lie group if it is locally compact and contains arbitrarily small compact normal subgroups $N$, such that $G / N$ is a finite-dimensional Lie group.

In order to understand the structure of pro-Lie groups in our sense, we developed a highly infinite-dimensional Lie theory of considerable complexity (see [5] and subsequent publications [6-8]). This Lie theory assigns to each pro-Lie group $G$ a pro-Lie algebra $\mathfrak{g}$ and an exponential function exp: $\mathfrak{g} \rightarrow G$. This approach was exploited very successfully in [9] for compact groups and was found to be an eminently useful extension of the classical theory of real Lie groups of finite dimension.

The theory of an $n$-dimensional real Lie group is based on the fact that open subsets of $\mathbb{R}^{n}$ have a rich differentiable structure that is transported to the group, allowing a differentiable multiplication and inversion in the group. It has been an ongoing effort to replace $\mathbb{R}^{n}$ by more general, possibly infinite-dimensional, topological vector spaces supporting differentiable structures. The most advanced such theory is the theory of Lie groups on differentiable or smooth manifolds modeled on open subsets of locally-convex vector spaces and their real analysis as used by Helge Glöckner and Karl-Hermann Neeb (see [10-12]). One may justifiably ask how the theory of pro-Lie groups and the theory of infinite-dimensional differentiable Lie groups in their spirit are related. It was shown in [13] that a pro-Lie group is a smooth Lie group in their sense if and only if it is locally contractible.

The theory of pro-Lie groups has been described in detail in the 678-page book [5] in 2007 and in later papers. An endeavor to summarize that work would therefore be futile. Rather, in this survey, we highlight central results and explain some key open problems.

The purely theoretical foundation of, and motivation for, the theory of pro-Lie groups, however, must be complemented by an outlook to areas in which they emerge naturally and by necessity. Section 8 therefore deals with the appearance of pro-Lie groups in the so-called character theory of Hopf algebras, which received attention in recent publications [14]. 


\section{The Topology of Pro-Lie Groups}

It has become a standard result in the literature that every connected locally-compact Abelian group is homeomorphic to $\mathbb{R}^{n} \times K$ where $K$ is a compact connected Abelian group (see, e.g., [9], Theorem 7.57). The non-Abelian version of this (see [15], p. 188f) says that a connected locally-compact group is homeomorphic to $\mathbb{R}^{n} \times K$, where $K$ is a compact connected group. We shall see in this section that a connected pro-Lie group is homeomorphic to $\mathbb{R}^{\mathbf{m}} \times K$ where $\mathbf{m}$ is an arbitrary cardinal and $K$ is a compact connected group. This is convincing evidence that connected pro-Lie groups represent a natural extension of connected locally-compact groups. We also see from this observation that the pro-Lie group $\mathbb{R}^{I}$, for an arbitrary set $I$, is a critical example of a pro-Lie group. We shall return to this theme repeatedly in this text.

We shall give now a complete description of the topological structure of an almost connected pro-Lie group [16]. We present it here because it is perhaps easily understood without too much background information. A complete proof of the result is far from elementary or short.

A compact connected group $S$ is said to be semisimple if its algebraic commutator subgroup is the whole group. Let us preface the main result by the remark that we know very explicitly the structure of compact connected semisimple groups from [9], Theorem 9.19. It is also a fact that in such a group, every element is itself a commutator.

Likewise, the structure of a compact connected Abelian group $A$ is well understood. Indeed, a compact Abelian group $A$ is connected if and only if its Pontryagin dual is a torsion-free Abelian group (see [9], Corollary 8.5). This allows the determination of details of the structure of such a group, as is expounded in [9], Chapter 8 .

We denote by $\mathbb{Z}(n)$ the $n$-element group $\mathbb{Z} / n \mathbb{Z}$.

With this notation and information at hand, one can appreciate the power of the following result:

Theorem 1. ([7], Corollary 8.9, p. 381) An almost connected pro-Lie group G contains a compact connected semisimple subgroup $S$ and a compact connected Abelian subgroup A, such that for suitable sets I and J, the topological group $G$ is homeomorphic to the topological group $\mathbb{R}^{I} \times S \times A \times \Delta$, where:

$$
\Delta= \begin{cases}\mathbb{Z}(n), & \text { if } G \text { has finitely many components, } \\ \mathbb{Z}(2)^{J}, & \text { otherwise. }\end{cases}
$$

This result allows several immediate corollaries, which are of interest for the topology of pro-Lie groups. 
Corollary 1. The space underlying an almost connected pro-Lie group is a Baire space.

This follows from Theorem 1 and Oxtoby's results in [17].

Corollary 2. Every almost connected pro-Lie group is homotopy equivalent to a compact group.

Indeed, $\mathbb{R}^{I}$ is homotopy equivalent to a singleton. The algebraic topology of an almost connected pro-Lie group therefore is that of a compact group (cf. [9]).

Corollary 3. An almost connected pro-Lie group is locally compact if and only if in Theorem 1, the set I is finite.

At the root of Theorem 1 is the following main theorem generalizing the Theorem on p. 188ff in [15].

Theorem 2. ([7], Main Theorem 8.1, p. 379) Every almost connected pro-Lie group G has a maximal compact subgroup $M$, and any other compact subgroup is conjugate to a subgroup of M. Moreover,

(1) $G=G_{0} M$,

(2) $M_{0}=G_{0} \cap M$, and

(3) $M_{0}$ is a maximal compact subgroup of $G_{0}$.

We record that the results of Theorem 2 enter into a proof of Theorem 1 in an essential way. In the process of proving Theorem 1 , one also establishes the following theorem, which is more concise than Theorem 1 if one assumes the structure theory of compact groups as presented in [9].

Theorem 3. ([7], Theorem 8.4) Let $G$ be an almost connected pro-Lie group, and $M a$ maximal compact subgroup of $G$. Then, $G$ contains a subspace $E$ homeomorphic to $\mathbb{R}^{I}$, for a set $I$, such that $(m, e) \mapsto m e: M \times E \rightarrow G$ is a homeomorphism. Thus, $G$ is homeomorphic to $\mathbb{R}^{I} \times M$.

\section{Pro-Lie Groups as Projective Limits of Lie Groups}

We have defined pro-Lie groups as closed subgroups of products of finite-dimensional real Lie groups. In fact, they can be equivalently defined as special closed subgroups of such products, namely projective limits of finite-dimensional Lie groups.

At first pass, this is surprising, and its proof requires some effort. 
Definition 1. A family $\left\{f_{j k}: G_{j} \rightarrow G_{k} \mid j, k \in J\right\}$ of morphisms of topological groups is called a projective system if $J$ is a directed set satisfying the conditions:

(a) for all $j \in J, f_{j j}$ is the identity map of $G_{j j}$, and

(b) for $i, j, k \in J$ with $i \leq j \leq k$, we have $f_{i k}=f_{i j} \circ f_{j k}$.

Given a projective system of morphisms of topological groups, we define a closed subgroup $G$ of the product $\prod_{j \in J} G_{j}$ to be the set $\left\{\left(g_{i}\right)_{i \in J}: g_{j}=f_{j k}\left(g_{k}\right)\right.$ for all $j \leq k$ in $\left.J\right\}$. This group $G$ is called the projective limit (of the projective system), denoted by $\lim _{j \in J} G_{j}$.

When we say in the following that a subgroup $N$ of a topological group $G$ is a co-Lie subgroup, we mean that $N$ is a normal closed subgroup, such that the factor group $G / N$ is a Lie group.

Theorem 4. ([5,18], Theorem 3.39 on p. 161, [6,19]) For a topological group G, the following conditions are equivalent.

(1) G is complete, and every identity neighborhood contains a co-Lie subgroup.

(2) $G$ is a projective limit of Lie groups.

(3) $G$ is a pro-Lie group.

This theorem, by the way, explains the choice of the name "pro-Lie group." (See also [20].) There is a considerable literature on the projective limits of finite discrete groups, called profinite groups (see [21,22]). Furthermore, amongst the pro-Lie groups, there are the prodiscrete groups, namely projective limits of discrete groups or, equivalently, closed subgroups of products of discrete groups. There is not much literature on prodiscrete groups. We formulate the following open question

Question 1: Is there a satisfactory structure theory for non-discrete prodiscrete groups? More particularly, is there a satisfactory structure theory even for Abelian non-discrete prodiscrete groups?

We do know that a quotient group of a pro-Lie group modulo a closed subgroup is a pro-Lie group if the quotient group is complete (see [5], Theorem 4.1.(i), p.170). One may reasonably ask whether, for a pro-Lie group $G$ and a closed normal subgroup $N$, we have sufficient conditions for $G / N$ to be complete and therefore a pro-Lie group (cf. [23]).

Theorem 5. ([5], Theorem 4.28, p. 202) Let $G$ be a pro-Lie group and $N$ a closed normal subgroup of $G$. Each of the following condition suffices for $G / N$ to be a pro-Lie group:

(i) $N$ is almost connected, and $G / G_{0}$ is complete.

(ii) $N$ satisfies the first axiom of countability.

(iii) $N$ is locally compact. 
The answer to following question is unknown:

Question 2: Let $G$ be a pro-Lie group with identity component $G_{0}$. Is $G / G_{0}$ complete (and therefore, prodiscrete)?

Indeed, this is the case when $G$ is Abelian; see Theorem 6(iii) below.

\section{Weakly-Complete Vector Groups}

In this section, we discuss the previously mentioned connected pro-Lie groups $\mathbb{R}^{I}$, for a set $I$. For infinite sets $I$, these are the simplest connected pro-Lie groups outside the class of locally-compact groups. However they will appear many times in the Lie theory we shall present. In particular, they feature in the structure theory of Abelian pro-Lie groups.

All vector spaces considered here are understood to be vector spaces over $\mathbb{R}$. For a vector space $E$, let $\operatorname{Hom}(E, \mathbb{R})$ denote the set of all linear functionals on $E$ with the vector space structure and topology it inherits from $\mathbb{R}^{E}$, the vector space of all functions $E \rightarrow \mathbb{R}$ with the product topology.

Proposition 1. For a topological vector space $V$, the following conditions are equivalent:

(1) There is a vector space $E$, such that the topological vector spaces $\operatorname{Hom}(E, \mathbb{R})$ and $V$ are isomorphic as topological groups.

(2) There is some set I, such that $V$ is isomorphic to $\mathbb{R}^{I}$ with the product topology.

Definition 2. A topological vector space $V$ is called weakly complete if it satisfies the equivalent conditions of Proposition 1.

Every weakly-complete vector space is a nuclear locally-convex space (see [24], p. 100, Corollary to Theorem 7.4, p. 103). The vector space $E$ in Proposition 1 is obtained as the vector space of all continuous linear maps $V \rightarrow \mathbb{R}$. In fact, the category of all weakly-complete vector spaces and continuous linear maps between them is dual to the category of all vector spaces and linear maps between them in a fashion analogous to the Pontryagin duality between compact Abelian groups and discrete Abelian groups (see [9], Theorem 7.30, [5], Appendix 2: Weakly Complete Topological Vector Spaces, pp. 629-650).

Theorem 6. ([5], Theorem 5.20, p. 230) For any Abelian pro-Lie group G, there is a weakly-complete vector subgroup $V$ and a closed subgroup $H$, such that (in additive notation):

(i) $(v, h) \mapsto v+h: V \times H \rightarrow G$ is an isomorphism of topological groups.

(ii) $H_{0}$ is a compact connected Abelian group, and every compact subgroup of $G$ is contained in $\mathrm{H}$.

(iii) $H / H_{0} \cong G / G_{0}$, and, thus, $G / G_{0}$ is prodiscrete. 
(iv) If $G_{a}$ and $H_{a}$ are the arc components of the identity of $G$ and $H$, respectively, then $G_{a}=V \oplus H_{a}$.

We note that in the present circumstances, the positive answer to Question 2 expressed in Conclusion (iii) follows from the compactness of $H_{0}$ via Theorem 5 .

We have seen that products of pro-Lie groups are pro-Lie groups and that closed subgroups of pro-Lie groups are pro-Lie groups. As a consequence, projective limits of pro-Lie groups are pro-Lie groups.

In Section 7 of [25], Banaszczyk introduced nuclear Abelian groups. Since all Abelian Lie groups are nuclear and the class of nuclear groups is closed under projective limits, all Abelian pro-Lie groups are nuclear. (See also [26].)

In these circumstances, it is somewhat surprising that quotient groups of pro-Lie groups may fail to be pro-Lie groups. Indeed, as we shall see in the next Proposition 2, there is a quotient group of a very simple Abelian pro-Lie group, namely of $\mathbb{R}^{2^{\aleph_{0}}}$, which is an Abelian topological group that is not complete and, therefore, is not a pro-Lie group. However, this situation is not as concerning as it might first appear, because every quotient group of a pro-Lie group has a completion, and the completion is a pro-Lie group.

We consider the unit interval $\mathbb{I}=[0,1]$ as representative for the sets of continuum cardinality $2^{\aleph_{0}}$. Let $\delta_{n} \in \mathbb{Z}^{(\mathbb{N})}$ be defined by:

$$
\delta_{n}=\left(e_{m n}\right)_{m \in \mathbb{N}}, \quad e_{m n}= \begin{cases}1 & \text { if } m=n \\ 0 & \text { otherwise }\end{cases}
$$

Then, $B=\left\{\delta_{n}: n \in \mathbb{N}\right\}$ generates the free Abelian group $\mathbb{Z}^{(\mathbb{N})}$ algebraically.

Proposition 2. ([5], Proposition 5.2, p. 214) The free Abelian group $\mathbb{Z}^{(\mathbb{N})}$ of countably-infinite rank has a non-discrete topology making it a prodiscrete group $F$, so that the following conditions are satisfied:

(i) F is a countable non-discrete non-metrizable pro-Lie group, which therefore is not a Baire space.

(ii) F can be considered as a closed subgroup of $V=\mathbb{R}^{\mathbb{I}}$ with dense $\mathbb{R}$-linear span in $V$, so that $V / F$ is an incomplete group whose completion is a compact-connected and locally-connected, but not arcwise-connected group.

(iii) Every compact subset of $F$ is contained in a finite rank subgroup.

The structure theory results we discussed permit us to derive results on the duality of Abelian pro-Lie groups. Recall that this class contains the class of all locally-compact Abelian groups properly and is contained in the class of all Abelian nuclear groups. 
For any Abelian topological group $G$, we let $\widehat{G}=\operatorname{Hom}(G, \mathbb{T})$ denote its dual with the compact open topology, where $\mathbb{T}=\mathbb{R} / \mathbb{Z}$ (see e.g., [9], Chapter 7). There is a natural morphism of Abelian groups $\eta_{G}: G \rightarrow \widehat{\widehat{G}}$ given by $\eta_{G}(g)(\chi)=\chi(g)$, which may or may not be continuous; information regarding this issue is to be found for instance in [9], notably in Theorem 7.7. We shall call an Abelian topological group semireflexive if $\eta_{G}: G \rightarrow \widehat{\widehat{G}}$ is bijective and reflexive if $\eta_{G}$ is an isomorphism of topological groups; in the latter case, $G$ is also said to have duality (see [9], Definition 7.8).

There is an example of a non-discrete, but prodiscrete Abelian torsion group due to Banaszczyk (see [25], p. 159, Example 17.11), which is semireflexive, but not reflexive (see also [5], Chapter 14, p. 595, Example 14.15; attention: in Line 2 of the text of this example, read $N_{\alpha}=\mathbb{Z}(2)(\{v: v \geq \alpha\})$, not $\left.v<\alpha\right)$. Therefore, we know that the category of Abelian pro-Lie groups is not self-dual under Pontryagin duality.

Theorem 7. ([5] Theorem 5.36, p. 239) Every almost connected Abelian pro-Lie group is reflexive, and its character group is a direct sum of the additive topological group of a real vector space endowed with the finest locally-convex topology and a discrete Abelian group. Pontryagin duality establishes a contravariant functorial bijection between the categories of almost connected Abelian pro-Lie groups and the full subcategory of the category of topological Abelian groups containing all direct sums of vector groups with the finest locally-convex topology and discrete Abelian groups.

By this theorem, duality applies to almost connected Abelian Lie groups. In particular, we recall that weakly-complete topological vector spaces have a good Pontryagin duality. By Theorem 7 above, the issue of duality of Abelian pro-Lie groups is reduced to groups with a compact identity component. Amongst this class there are all prodiscrete groups. In particular, nothing is known about the duality of prodiscrete Abelian groups. As all Abelian pro-Lie groups are nuclear, whatever is known on the duality of nuclear Abelian groups applies to pro-Lie groups.

Question 3: Which Abelian pro-Lie groups are reflexive?

Question 4: Which Abelian pro-Lie groups are strongly reflexive in the sense that all of their subgroups and Hausdorff quotient groups are reflexive?

\section{The Open Mapping Theorem}

We have just dealt with the question of whether a quotient group of a pro-Lie group is a pro-Lie groups, and we have seen that the answer is negative sometimes. We now deal with the question when a surjective morphism of pro-Lie groups is a quotient morphism. Specifically, let $f: G \rightarrow H$ be a surjective morphism of pro-Lie groups. Does this imply that $f$ is an open mapping? This question is answered negatively in any first course on topological groups by the example 
of $G=\mathbb{R}_{d}$, the additive group of reals with the discrete topology and $H=\mathbb{R}$ with its natural topology. The quest for sufficient conditions that would secure the openness is answered by any of the so-called "open mapping theorems" in the classical literature in functional analysis and in the theory of topological groups. These impose countability conditions on $G$ and a Baire space hypothesis on $H$. The latter is provided by such properties as complete metrizability, local compactness or the pro-Lie group property. If $\sigma$-compactness is taken as a countability condition on $G$, then the Baire space property of $H$ will force local compactness upon $G / \operatorname{ker} f$. The induced bijection $G / \operatorname{ker} f \rightarrow H$ is an isomorphism if and only if $f$ is open. Therefore, settling the issue for bijective $f$ cannot be much of a restriction, assuming that the properties envisaged for $G$ are preserved by passing to quotients.

Whichever way the issue is looked at, the proof of an open mapping theorem for pro-Lie groups $[27,28]$ is quite different from all other open mapping theorems we know.

Once more, we encounter the class of almost connected pro-Lie groups as that class on which our methods, notably a Lie theory, which we have yet to discuss, yields an expected result.

Theorem 8. ([5], 9.60, p.409f) [6,19]) Let $G$ and $H$ be pro-Lie groups, and let $f: G \rightarrow H$ be a surjective continuous homomorphism. Then, $f$ is an open mapping if $G$ is almost connected. In particular, the natural bijective morphism $G / \operatorname{ker} f \rightarrow H$ is an isomorphism of topological groups.

The last conclusion yields another instance of a quotient group of a pro-Lie group, which is again a pro-Lie group, giving us a sufficient condition not included in Theorem 5 above, namely $G$ is almost connected, and $N$ is the kernel of a morphism onto a pro-Lie group.

A further corollary of our open mapping theorem is the following form of the second isomorphism theorem for pro-Lie groups:

Corollary 4. Assume that $N$ and $H$ are two closed almost connected subgroups of a topological group with $N$ being normal, and assume that $N H$ is a pro-Lie group. Then, $H /(N \cap H) \cong N H / N$. Moreover, the natural morphism $\mu: N \rtimes H \rightarrow N H, m u(n, h)=$ $n h$ is a quotient morphism (where $H$ is acting as an automorphism group on $N$ via inner automorphisms.

Noting that $\mathbb{Z}^{I}$ is a pro-Lie group, but is not Polish, unless $I$ is countable (see [5], pp. 235, 236, notably Lemma 5.30), we ask:

Question 5: Is a surjective morphism $f: \mathbb{Z}^{I} \rightarrow K$ onto a compact group open for every set $I$ ? 
The results in Theorem 8 and in Chapter 5 of [5] do not answer this question. If $I$ is countable, then $\mathbb{Z}^{I}$ is a Polish group, and an open mapping theorem applies in this case and gives an affirmative answer. The open mapping theorem for Pro-Lie groups does not apply, since $\mathbb{Z}^{I}$ is never almost connected for I nonempty.

Added in Proof: Saak Gabriyelyan and the second author have recently announced a positive answer to Question 5 .

\section{Lie Theory}

We started our discussion with a presentation of some remarkable structure theorems on almost connected pro-Lie groups. It is not surprising that the proofs of such results require some powerful tools. The crucial tool for a structure theory of pro-Lie groups is their Lie theory. It is a challenge to explain what we mean by "Lie theory." Minimally, one wants to attach to each pro-Lie group $G$ a Lie algebra $\mathfrak{L}(G)$ with characteristics making it suitable for an exploitation of its topological algebraic structure for the topological group structure of the pro-Lie group $G$. Guided by our knowledge of classical Lie group theory, we shall link the group $G$ with its Lie algebra $\mathfrak{L}(G)$ by an exponential function exp: $\mathfrak{L}(G) \rightarrow G$, which mediates between Lie algebra theoretical properties of $\mathfrak{L}(G)$ and group theoretical properties of $G$.

As a start, for each $X \in \mathfrak{L}(G)$, the function $t \mapsto \exp (t . X): \mathbb{R} \rightarrow G$ is a morphism of topological groups. Tradition calls a morphism $f: \mathbb{R} \rightarrow G$ of topological groups a one-parameter subgroup of $G$ (admittedly, a misnomer). In classical Lie theory, every one-parameter subgroup is obtained via the exponential function in this fashion. In other words, however one defines a Lie algebra and an exponential function, it must establish a bijection $\beta$ from the elements of the Lie algebra $\mathfrak{L}(G)$ to the set $\operatorname{Hom}(\mathbb{R}, G)$ of one-parameter subgroups of $G$, so that the following diagram commutes:

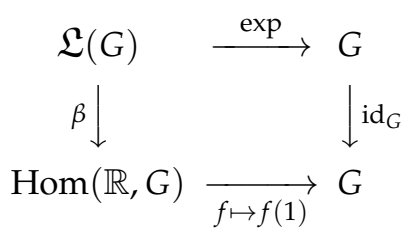

Therefore, if we are given a topological group $G$, as a first step, we may think of $\mathfrak{L}(G)$ as $\operatorname{Hom}(\mathbb{R}, G)$ with a scalar multiplication, such that $r \cdot X(s)=X(s r)$ for $X \in \mathfrak{L}(G)$ and $r, s \in \mathbb{R}$. If $G$ has an additional structure, such as that of a pro-Lie group, we will obtain the additional structure on $\mathfrak{L}(G)$. Therefore, if $G$ is a closed subgroup of a product $P=\prod_{i \in I} G_{i}$ of finite-dimensional Lie groups, then an element $X \in \mathfrak{L}(P)$ may be identified with an element $\left(X_{i}\right)_{i \in I}$ of $\prod_{i \in I} \mathfrak{L}\left(G_{i}\right)$, and an element $X$ of this kind is in $\mathfrak{L}(G)$ simply if $X(\mathbb{R}) \subseteq G$. As $G_{i}$ is a finite-dimensional Lie group, each $\mathfrak{L}\left(G_{i}\right)$ has the structure of a Lie algebra, $\mathfrak{L}(P)$ is both a weakly-complete topological vector space and a topological Lie algebra. Since $G$ is a closed subgroup 
of $P$, it is elementary that $\mathfrak{L}(G)$ is closed in $\mathfrak{L}(P)$, both topologically and in the sense of Lie algebras.

Definition 3. A pro-Lie algebra $\mathfrak{g}$ is a topological real Lie algebra isomorphic to a closed subalgebra of a product of finite-dimensional Lie algebras.

Clearly, every pro-Lie algebra has a weakly-complete topological vector space as the underlying topological vector space. In complete analogy to Theorem 4, we have the following characterization [29]:

Theorem 9. ([5], p. 138ff) For a topological Lie algebra $\mathfrak{g}$, the following conditions are equivalent:

(1) $\mathfrak{g}$ is complete, and every neighborhood of zero contains a closed ideal $\mathfrak{i}$, such that the Lie algebra $\mathfrak{g} / \mathfrak{i}$ is finite-dimensional.

(2) $\mathfrak{g}$ is a projective limit of finite-dimensional Lie algebras.

(3) $\mathfrak{g}$ is a pro-Lie algebra.

One notes that our procedure of identifying $\mathfrak{L}(G)=\operatorname{Hom}(\mathbb{R}, G)$ for a pro-Lie group $G$ with the structure of a pro-Lie algebra yields an exponential function $\exp : \mathfrak{L}(G) \rightarrow G$ by $\exp X=X(1)$ for $X: \mathbb{R} \rightarrow G$ in $\mathfrak{L}(G)$. The implementation of this setup is secured in [5], summarized in the following:

Theorem 10. To each pro-Lie group, there is uniquely and functorially assigned a pro-Lie algebra $\mathfrak{L}(G)$ together with an exponential function $\exp _{G}: \mathfrak{L}(G) \rightarrow G$, such that every one-parameter subgroup of $G$ is of the form $t \mapsto \exp t . X: \mathfrak{L}(G) \rightarrow G$ with a unique element $X \in \mathfrak{L}(G)$ and that the subgroup $\langle\exp \mathfrak{L}(G)\rangle$ generated by all one parameter subgroups is dense in the identity component $G_{0}$ of the identity in $G$.

To each pro-Lie algebra $\mathfrak{g}$ there is a uniquely-and functorially-assigned connected pro-Lie group $\Gamma(\mathfrak{g})$ with Lie algebra $\mathfrak{g}$, and for each pro-Lie group $G$ with Lie algebra $\mathfrak{L}(G)$ permitting an isomorphism $f: \mathfrak{g} \rightarrow \mathfrak{L}(G)$ of pro-Lie algebras, there is a unique isomorphism of pro-Lie groups $f_{\Gamma}: \Gamma(\mathfrak{g}) \rightarrow G$, such that the following diagram is commutative with exp denoting the exponential function of $\Gamma(\mathfrak{g})$ :

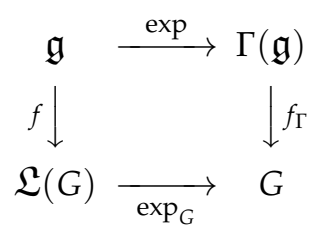

A pro-Lie group $G$ is prodiscrete if and only if it is totally disconnected if and only if $\mathfrak{L}(G)=\{0\}$. Further, $\Gamma(\mathfrak{g})$ is always simply connected. 
Sophus Lie's third theorem applies and is perfectly coded into the existence of the functor $\Gamma$. The exactness properties of the functor $\mathfrak{L}$ are well understood (see [5], Theorem 4.20, p. 188). Structural results, such as we discussed at the beginning of our survey, are all based on a thorough application of the Lie theory of pro-Lie groups. Since $\mathfrak{L}(G)=\mathfrak{L}\left(G_{0}\right)$ from the very definition of $\mathfrak{L}(G)$, in the strictest sense it applies to connected pro-Lie groups, we saw that the essential facts reach out to almost connected pro-Lie groups.

Of course, since every locally-compact group has an open subgroup that is an almost connected pro-Lie group, this Lie theory applies to all locally-compact groups.

In classical Lie theory, Lie algebras are more directly amenable to structural analysis than Lie groups, as they are purely algebraic. While pro-Lie algebras are both topological and algebraic, they are nevertheless more easily analyzed than pro-Lie groups, as well.

Let us look as some characteristic features of pro-Lie algebras.

Definition 4. A pro-Lie algebra $\mathfrak{g}$ is called:

(i) reductive if every closed ideal is algebraically and topologically a direct summand,

(ii) prosolvable if every finite-dimensional quotient algebra is solvable,

(iii) pronilpotent if every finite-dimensional quotient algebra is nilpotent.

The center of $\mathfrak{g}$ is denoted $\mathfrak{z}(\mathfrak{g})$. A pro-Lie algebra is called semisimple if it is reductive and satisfies $\mathfrak{z}(\mathfrak{g})=\{0\}$.

Theorem 11. ([5] Theorem 7.27, p. 283) A reductive pro-Lie algebra $\mathfrak{g}$ is a product of finite-dimensional ideals, each of which is either simple or else is isomorphic to $\mathbb{R}$.

The algebraic commutator algebra $[\mathfrak{g}, \mathfrak{g}]$ is closed and a product of simple ideals. Furthermore,

$$
\mathfrak{g}=\mathfrak{z}(\mathfrak{g}) \oplus[\mathfrak{g}, \mathfrak{g}]
$$

algebraically and topologically.

If a maximal prosolvable (respectively, pronilpotent) ideal of a pro-Lie algebra $\mathfrak{g}$ exists, it is called the (solvable) radical $\mathfrak{r}(\mathfrak{g})$ (respectively, the nilradical $\mathfrak{n}(\mathfrak{g})$ ). If there is a smallest closed ideal $\mathfrak{i}$ of $\mathfrak{g}$, such that $\mathfrak{g} / \mathfrak{i}$ is reductive, then we call it the co-reductive radical $\mathfrak{n}_{\text {cored }}(g)$ of $\mathfrak{g}$.

Theorem 12. ([5], Chapter 7) Every pro-Lie algebra has a radical, a nilradical and a co-reductive radical, and:

$$
\mathfrak{n}_{\text {cored }}(g) \subseteq \mathfrak{n}(\mathfrak{g}) \subseteq \mathfrak{r}(\mathfrak{g})
$$


Moreover,

$$
\mathfrak{n}_{\text {cored }}(\mathfrak{g})=\overline{[\mathfrak{g}, \mathfrak{g}]} \cap \mathfrak{r}(\mathfrak{g})=\overline{[\mathfrak{g}, \mathfrak{r}(\mathfrak{g})] .}
$$

There is a closed semisimple subalgebra $\mathfrak{s}$, such that $\mathfrak{g}=\mathfrak{r}(\mathfrak{g}) \oplus \mathfrak{s}$, where $(r, s) \mapsto$ $r+s: \mathfrak{r}(\mathfrak{g}) \times \mathfrak{s} \rightarrow \mathfrak{g}$ is an isomorphism of weakly-complete topological vector spaces (Levi-Mal'cev decomposition). Moreover,

$$
\overline{[\mathfrak{g}, \mathfrak{g}]}=\mathfrak{n}_{\text {cored }}(\mathfrak{g}) \oplus \mathfrak{s} .
$$

All of this fine structure can be translated to the group level with due circumspection and ensuing complications. One can get an idea of this translation from the process of Lie's third theorem. Among other things, Theorem 10 yields for each of the pro-Lie algebras $\mathfrak{g}$ a pro-Lie group $\Gamma(\mathfrak{g})$ with an exponential function $\exp : \mathfrak{g} \rightarrow \Gamma(\mathfrak{g})$.

If $\mathfrak{g}$ is pronilpotent, then exp is a homeomorphism. In fact, the Baker-CampbellHausdorff series is summable on the weakly-complete topological vector space $\mathfrak{g}$ yielding a binary operation $\star$, so that for $\Gamma(\mathfrak{g})$, we may take $(\mathfrak{g}, \star)$ and for exp the identity map. This applies, in particular, to the co-reductive radical $\mathfrak{n}_{\text {cored }}(\mathfrak{g})$, for which $\mathfrak{g} / \mathfrak{n}_{\text {cored }}(\mathfrak{g})$ is reductive.

For reductive $\mathfrak{g}$, however, the product structure of $\mathfrak{g}$ expressed in Theorem 10 carries over to a clean product structure:

$$
\Gamma(\mathfrak{g}) \cong \mathbb{R}^{I} \times \prod_{j \in J} S_{j}
$$

for a family of simply-connected simple real Lie groups $S_{j}$, producing, in fact, a simply-connected reductive group $\Gamma(\mathfrak{g})$.

These observations show again how far connected pro-Lie groups reach outside the domain of locally-compact connected groups while their structure remains close to that which is familiar from finite-dimensional Lie groups due to a fairly lucid topological-algebraic structure of pro-Lie algebras. We note that for every connected pro-Lie group $G$ with $\mathfrak{L}(G) \cong \mathfrak{g}$, one has a morphism $f: \Gamma(\mathfrak{g}) \rightarrow G$ with a prodiscrete kernel and a dense image, such that the following diagram is commutative:

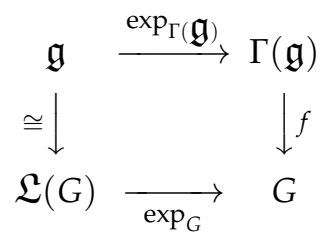

In [5], it is demonstrated that this tool allows a structural analysis of $G$.

For instance, the existence of the various radicals of a pro-Lie algebra has its correspondence in respective radicals in any connected pro-Lie group. For example, 
every connected pro-Lie group $G$ has a unique largest normal connected solvable subgroup $\mathbf{R}(G)$, called its (solvable) radical. If $G$ is any topological group whose identity component $G_{0}$ is a pro-Lie group, then one writes $\mathbf{R}(G)=\mathbf{R}\left(G_{0}\right)$ and also calls $\mathbf{R}(G)$ the radical of $G$.

The volume of additional details of the theory of pro-Lie algebras and connected pro-Lie groups presented in [5] is immense. It cannot be expected that a survey such as this can do complete justice to it.

\section{Later Developments}

In this section, we report on some developments in the theory of pro-Lie groups since the appearance of [5]. Of course, we have already included some important material, which appeared subsequent to [5], namely [7,13].

The article [7] contributed the insight that some essential structure theorems on connected pro-Lie groups could be formulated so as to include almost connected pro-Lie groups. This provided a common generalization of the structure theories of connected pro-Lie groups and compact groups. This generalization is both significant and satisfying. In this survey, this was illustrated by Theorem 1 and its corollaries and Theorems 2 and 3.

Hofmann-Morris [7] contains another interesting result, which we think has yet to be exploited in the literature.

Theorem 13. Let $G$ be an arbitrary topological group whose identity component $G_{0}$ is a pro-Lie group. Then, there is a closed subgroup $G_{1}$ whose identity component is the radical $\mathbf{R}(G)$, such that the following conditions hold:

(i) $G=G_{0} G_{1}$ and $G_{0} \cap G_{1}=\mathbf{R}(G)$.

(ii) The factor group $G / \mathbf{R}(G)$ is the semidirect product of the connected normal subgroup $G_{0} / \mathbf{R}(G)$ and the totally disconnected closed subgroup $G_{1} / \mathbf{R}(G)$.

(iii) In particular,

$$
\frac{G}{G_{0}} \cong \frac{G / \mathbf{R}(G)}{G_{0} / \mathbf{R}(G)}=\frac{G / \mathbf{R}\left(G_{0}\right)}{G_{0} / \mathbf{R}\left(G_{0}\right)} \cong \frac{G_{1}}{\left(G_{1}\right)_{0}}
$$

with a prosolvable pro-Lie group $\left(G_{1}\right)_{0}$.

The significance of Theorem 13 emerges even when it is specialized to the case that $G$ is a pro-Lie group. As was emphasized by formulating Question 2, we do not know whether the component factor group $G / G_{0}$ is complete and, therefore, is a prodiscrete group. Theorem 13 reduces the problem to the case that the identity component of $G$ is prosolvable. For instance, we obtain a positive answer to Question 2 if we know that the radical $\mathbf{R}(G)$ is locally compact or first countable (see Theorem 5 above). 
In the process of extending the structure theory of pro-Lie groups from connected ones to almost connected ones, G. Michael, A.A. has proven the following structure theorem guaranteeing a local splitting, provided the nilradical is not too big on the Lie algebra side.

Theorem 14. ([8,30]) Assume that $G$ is an almost connected pro-Lie group $G$ whose Lie algebra $\mathfrak{g}$ has a finite-dimensional co-reductive radical $\mathfrak{n}_{\text {cored }}(\mathfrak{g})$. Then, there are arbitrarily small closed normal subgroups $N$, such that there exists a simply-connected Lie group $L_{N}$ and a morphism $\alpha: L_{N} \rightarrow G$, such that the morphism:

$$
(n, x) \mapsto n \alpha(x): N \times L_{N} \rightarrow G
$$

is open and has a discrete kernel. In particular, $G$ and $N \times L_{N}$ are locally isomorphic.

Let us recall Iwasawa's local splitting theorem for locally-compact groups as it is reported in [9], Theorem 10.89.

Theorem 15. Let $G$ be a locally-compact group. Then, there exists an open almost connected subgroup $A$, such that for each identity neighborhood $U$, there is:

- a compact normal subgroup $N$ of $A$ contained in $U$,

- a simply-connected Lie group L, and

- an open and continuous surjective morphism $\phi: N \times L \rightarrow A$ with a discrete kernel, such that $\phi(n, 1)=n$ for all $n \in N$.

The way to compare the two preceding theorems is to look at the Lie algebra $\mathfrak{g}$ of $G$ in Theorem 15. We notice that $\mathfrak{g}=\mathfrak{L}(N) \oplus \mathfrak{l}, \mathfrak{l}=\mathfrak{L}(L)$. As the Lie algebra of a compact group $N$ of the first direct summand is of the form $\mathfrak{L}(N)=\mathfrak{c} \oplus \mathfrak{s}_{1}$ with a central ideal $\mathfrak{c}$ and a compact semisimple ideal $\mathfrak{s}_{1}$, the finite-dimensional Lie algebra $\mathfrak{l}$ has a Levi-Mal'cev decomposition $\mathfrak{r} \oplus \mathfrak{s}_{2}$ with its radical $\mathfrak{r}$ and a finite-dimensional semisimple subalgebra $\mathfrak{s}_{2}$, so that we have:

$$
\mathfrak{g}=\left(\mathfrak{c} \oplus \mathfrak{s}_{1}\right) \oplus\left(\mathfrak{r}+_{\text {sdir }} \mathfrak{s}_{2}\right)=(\mathfrak{r} \oplus \mathfrak{c})+_{\text {sdir }}\left(\mathfrak{s}_{1}+\mathfrak{s}_{2}\right)
$$

We observe that the radical $\mathfrak{r}(\mathfrak{g})$ is $\mathfrak{r} \oplus \mathfrak{c}$ and that the co-reductive radical $\mathfrak{n}_{\text {cored }}(\mathfrak{g})$ of $\mathfrak{g}$ are contained in the finite-dimensional subalgebra $\mathfrak{r}$ and are, therefore, finite-dimensional. The hypothesis that was imposed in Theorem 14, namely that the Lie algebra dimension of the co-reductive radical is finite, thus emerges as a necessary condition in the more classical Iwasawa local decomposition theorem of locally-compact groups. Additional comments on the local decomposition of pro-Lie groups may be found in [31]. 


\section{A Natural Occurrence of Pro-Lie Groups and Pro-Lie Algebras}

We emphasized that weakly-complete topological vector spaces play an essential role in the theory of pro-Lie groups and pro-Lie algebras. Now, we record that they are crucial in describing a mathematical environment where they occur naturally; this was pointed out recently in [14]. Each of the categories of real vector spaces and of their dual category of weakly-complete topological vector spaces (see Section 3, Definition 3.2ff) in fact has a tensor product (see [14], Appendix C, notably C4, and [32]), making each of them a commutative monoidal category (see, e.g., [9], Appendix 3, Definition A3.62 ff). Let us denote by $\mathcal{V}$ the category of (real) vector spaces $E, F \ldots$, and of linear maps, equipped with the usual tensor product $E \otimes F$, and let us call $\mathcal{W}$ the category of weakly-complete vector spaces $V, W, \ldots$, etc., with continuous linear maps, equipped with the (completed) tensor product $V \widetilde{\otimes} W$. Then, a monoid in $\mathcal{W}$ (see [9] Appendix 3, the discussion preceding Definition A3.64) is a weakly-complete topological (associative) algebra with identity, specifically, a morphism $m: A \widetilde{\otimes} A \rightarrow A$ plus a morphism $\mathbb{R} \rightarrow A$ representing the identity (see also [32]). Its dual $E \stackrel{\text { def }}{=} A^{\prime}=\operatorname{Hom}(A, \mathbb{R})$ then is an (associative unital) coalgebra $m^{\prime}: E \rightarrow E \otimes E$ with a coidentity (augmentation) $u: E \rightarrow \mathbb{R}$. The theory of coalgebras (see [33]) culminates in one theorem holding without any further hypotheses:

Theorem 16. (The fundamental theorem of coalgebras [33], 4.12, p. 742) Each associative unitary coalgebra is the directed union of its finite-dimensional unitary sub-coalgebras.

By duality this implies at once Rafael Dahmen's fundamental theorem of weakly-complete algebras.

Theorem 17. (Fundamental theorem of weakly-complete algebras [14]) For every weakly-complete associative unital algebra $A$, there is a projective system of surjective linear morphisms $f_{j k}: A_{k} \rightarrow A_{j}, j \leq k, j, k \in J$ of finite-dimensional associative unital algebras and a natural isomorphism $\phi_{A}: A \rightarrow \lim _{j \in J} A_{j}$ onto the projective limit of this system.

Conversely, by definition, every projective limit of finite-dimensional real unital associative algebras is a weakly-complete associative unital algebra.

Every associative algebra $A$ becomes a Lie algebra $A_{\text {Lie }}$ when it is equipped with the commutator bracket $(x, y) \mapsto[x, y] \stackrel{\text { def }}{=} x y-y x$. Each of the Lie algebras $\left(A_{j}\right)_{\text {Lie }}$ with $j \in J$ is finite-dimensional. From Theorem 17 and the Definition 3 plus Theorem 9, we thus have:

Corollary 5. For every weakly-complete associative unital algebra $A$, the Lie algebra $A_{\text {Lie }}$ is a pro-Lie algebra, and $\phi_{A}: A_{\mathrm{Lie}} \rightarrow \lim _{j \in J}\left(A_{j}\right)_{\mathrm{Lie}}$ is an isomorphism of pro-Lie algebras. 
Each of the morphisms $f_{j k}$ maps the group $A_{k}^{-1}$ of units, that is invertible elements, into the group of units $A_{j}^{-1}$, and so, we obtain a natural isomorphism:

$$
\phi_{A} \mid A^{-1}: A^{-1} \rightarrow \lim _{j \in J} A_{j}^{-1}
$$

Now, every $A_{j}^{-1}$ is a (linear) Lie group (see [5], Chapter 5, 5.1-5.32) with exponential function $\exp _{A_{j}}:\left(A_{j}\right)_{\text {Lie }} \rightarrow A_{j}^{-1}$. By Theorem 4 , as a consequence, we have Dahmen's corollary.

Corollary 6. ([14], Proposition 5.4) The group $A^{-1}$ of units of every weakly-complete associative unital algebra $A$ is a pro-Lie group with Lie algebra $A_{\text {Lie }}$ and exponential function:

$$
\exp _{A}: A_{\text {Lie }} \rightarrow A^{-1}, \quad \exp (x)=1+x+\frac{1}{2 !} x^{2}+\frac{1}{3 !} x^{3}+\cdots
$$

What we have exposed here is the basis of a theory that applies to group objects in the commutative monoidal category $\mathcal{W}$ as defined in [9], Definition A3.64(ii). These objects are commonly called Hopf algebras, and so, we shall fix the following definition.

Definition 5. A weakly-complete Hopf algebra is a group object in $\mathcal{W}$ according to Definition A3.64(ii) of [9].

In particular, the multiplicative structure of a weakly-complete Hopf algebra $A$ is a weakly-complete associative unital algebra. It also has a comultiplication $c: A \rightarrow$ $A \widetilde{\otimes} A$ linked with the multiplication $m: A \widetilde{\otimes} A \rightarrow A$ through several commutative diagrams, for which we refer to [9], A3.63(ii), and which express the fact that $c$ is indeed a morphism of algebras.

Definition 6. Let $A$ be a weakly-complete Hopf algebra with a comultiplication c. An element $x \in A$ is called group-like if it satisfies $c(x)=x \widetilde{\otimes} x$ and $u(x)=1$, and it is called primitive if it satisfies $c(x)=x \widetilde{\otimes} 1+1 \widetilde{\otimes} x$. The set of group-like (respectively, primitive) elements will be denoted by $G(A)$ (respectively, $P(A)$ ).

One shows the following fact:

Theorem 18. $G(A)$ is a closed subgroup of $A^{-1}$, the group of units of the underlying algebra, and $P(A)$ is a closed Lie subalgebra of $A_{\text {Lie. }}$.

The link to the previous remarks is provided by the following theorem: 
Theorem 19. Let $A$ be a weakly-complete Hopf algebra. Then, the set of group-like elements $G(A)$ is a pro-Lie group, and the set of primitive elements $P(A)$ is a pro-Lie algebra and is the Lie algebra of $G(A)$ with the exponential function:

$$
\exp _{A} \mid P(A): P(A) \rightarrow G(A)
$$

with the restriction of the exponential function of Corollary 5.

A proof is based on the fact that for the algebra morphism $c$, we have $\exp _{A} \circ c=c \circ\left(\exp _{A} \widetilde{\otimes} \exp _{A}\right)$. If $x$ is primitive, then $c(x)=(x \widetilde{\otimes} 1)+(1 \widetilde{\otimes} x)$, and thus, $c(\exp x)=\exp (c(x))=\exp ((x \widetilde{\otimes} 1)+(1 \widetilde{\otimes} x))=(\exp x \widetilde{\otimes} 1)(1 \widetilde{\otimes} \exp x)=$ $\exp x \widetilde{\otimes} \exp x$. Therefore, exp maps $P(A)$ into $G(A)$. To see the converse, let $t \mapsto \exp t \cdot x: \mathbb{R} \rightarrow G(A)$ be a one-parameter subgroup. Then, $\exp t \cdot x$ is group-like for all $t$, i.e.,

$$
\begin{aligned}
\exp t \cdot c(x) & =\exp c(t \cdot x)=c(\exp t \cdot x)=(\exp t \cdot x) \widetilde{\otimes}(\exp t \cdot x) \\
& =((\exp t \cdot x) \widetilde{\otimes} 1)(1 \widetilde{\otimes}(\exp t \cdot x)) \\
& =\exp (t \cdot x \widetilde{\otimes} 1) \exp (1 \widetilde{\otimes} t \cdot x)=\exp ((t \cdot x \widetilde{\otimes} 1)+(1 \widetilde{\otimes} t \cdot x)) \\
& =\exp t \cdot(x \widetilde{\otimes} 1+1 \widetilde{\otimes} x), \quad \text { for all } t \in \mathbb{R},
\end{aligned}
$$

and this implies:

$$
c(x)=x \widetilde{\otimes} 1+1 \widetilde{\otimes} x
$$

which means $x \in P(A)$.

If the weakly-complete Hopf algebra $A$ arises as the dual of an (abstract) Hopf algebra $H$ (i.e., a group object in $\mathcal{V}$ ), then the members of $G(A)$ are multiplicative linear functionals on $H$, the so-called characters of $H$. Thus, Theorem 19 may be interpreted as saying that the character group of a Hopf algebra is a pro-Lie group (see also Theorem 5.6 in [14]).

The simplest example is $A=\mathbb{R}[[x]]$, the algebra of formal power series in one variable. As a vector space, $A$ is isomorphic to $\mathbb{R}^{\left\{1, x, x^{2}, \ldots\right\}}$, and this is weakly complete. Then, $A \widetilde{\otimes} A$ is isomorphic to $\mathbb{R}[[y, z]]$, the formal power series algebra in two commuting variables $y$ and $z$. The algebra morphism $\mathbb{R}[[x]] \rightarrow \mathbb{R}[[y, z]]$ generated by $x \mapsto y+z$ gives a comultiplication $c: A \rightarrow A \widetilde{\otimes} A$ making $A$ into a weakly-complete Hopf algebra. The multiplicative subgroup $G(A)=\{\exp t \cdot x: t \in \mathbb{R}\}$ is a Lie group isomorphic to $(\mathbb{R},+)$, and $P(A) \cong \mathbb{R} \cdot x$ is (trivially) a Lie algebra mapped by exp onto $G(A)$.

Question 6: (i) Is there a more elaborate duality theory of real Hopf algebras and weakly-complete Hopf algebras in which these facts on pro-Lie group and pro-Lie algebra theory play a role? 
(ii) Does the existence of weakly-complete enveloping algebras of weaklycomplete Lie algebras secure for each pro-Lie algebra $L$, an associative weaklycomplete Hopf Algebra $U(L)$, such that $L$ is isomorphic to a closed Lie subalgebra of $P(U(L))$ ?

\section{Further Open Questions}

In this last section, we record a few additional questions on pro-Lie groups that do not fit naturally with the material previously discussed in this paper, but that are of some significance to pro-Lie group theory. In so doing, we rely on definitions and concepts defined and discussed in [5].

Question 7: Is an Abelian prodiscrete compactly-generated group without nondegenerate compact subgroups a discrete group?

For a a definition and discussion of compactly-generated groups, see [5], Definition 5.6, pp. 218ff. For a discussion of Abelian compactly generated pro-Lie groups, see [5], Theorem 5.32, p. 236.

Question 8: Is a compactly-generated Abelian prodiscrete compact-free group a finitely-generated-free Abelian group?

Note that by compact free we mean the group has no nontrivial compact subgroups.

See [5], Theorem 5.32, the Compact Generation Theorem for Abelian Pro-Lie Groups, p. 236.

In the proof of the structure of reductive pro-Lie algebras in Theorem 11 ([5], Theorem 7.27), one uses the lemma that in every finite-dimensional real semisimple Lie algebra every element is a sum of at most two Lie brackets. For brackets in semisimple Lie algebras, see [5], Appendix 3, p. 651ff.

Question 9: Is every element in an arbitrary real semisimple Lie algebra a bracket?

For the concept of transfinitely-solvable pro-Lie algebras and pro-Lie groups, see [5], Definition 7.32, pp. 285ff, respectively pp. 420ff. For the concept of transfinitely-nilpotent pro-Lie algebras and pro-Lie groups, see [5], pp. 296ff, respectively, pp. $443 \mathrm{ff}$.

Question 10: Is a transfinitely-nilpotent connected pro-Lie group pronilpotent?

In Question 10, such a group has to be prosolvable, since it is transfinitely solvable, and then, the Equivalence Theorem for Solvability of Connected Pro-Lie Groups 10.18 of [5] applies. The impediment to a proof is the failure of transfinite nilpotency to be preserved by passing to quotients. Free topological groups are free groups in the algebraic sense and, thus, are countably nilpotent; but, every topological group is a quotient of a free topological group and, thus, of a transfinitely-countably nilpotent topological group.

For the definition of an analytic subgroup, see [5], Definition 9.5 on p. 360.

Question 11: Let $\mathfrak{h}$ be a closed subalgebra of the Lie algebra $\mathfrak{g}$ of a connected pro-Lie group $G$. Let $A(\mathfrak{h})$ denote the analytic group generated by $\mathfrak{h}$. Is $\overline{A(\mathfrak{h})} / A(\mathfrak{h})$ Abelian? 
(See [5], Theorem 9.32.)

Question 12: Is there a satisfactory theory of Polish pro-Lie groups (respectively, separable pro-Lie groups, or compactly-generated pro-Lie groups), notably in the connected case?

For information in [5] on the Abelian case, see pp. 235ff.

Acknowledgments: We gratefully acknowledge helpful and constructive conversations we had with Saak S. Gabriyelyan (Be'er Sheva, Israel) and Rafael Dahmen (Darmstadt, Germany), who also provided valuable assistance in locating typographical errors in earlier versions of our manuscript. We also thank the referees for finding typographical errors. Finally, the second author thanks the Technische Universität Darmstadt for hospitality, during which time the first draft of this survey article was written.

Author Contributions: Karl H. Hofmann and Sidney A. Morris each contributed $50 \%$ to this publication.

Conflicts of Interest: The authors declare no conflict of interest.

\section{References}

1. Tao, T. Hilbert's Fifth Problem and Related Topics; Amer. Math. Soc.: Providence, RI, USA, 2014.

2. Hofmann, K.H.; Morris, S.A. On a category of topological groups suitable for a structure theory of locally compact groups. Topology Proc. 2001-2002, 26, 651-665.

3. Hofmann, K.H.; Morris, S.A. Projective limits of finite dimensional Lie groups. Proc. London Math. Soc. 2003, 87, 647-676.

4. Hofmann, K.H.; Morris, S.A. Lie theory and the structure of pro-Lie groups and Pro-Lie Algebras. Topology Proceedings 2004, 28, 541-567.

5. Hofmann, K.H.; Morris, S.A. The Lie Theory of Connected Pro-Lie Groups; European Mathematical Society: Zürich, Switzerland, 2007.

6. Hofmann, K.H.; Morris, S.A. On the Pro-Lie Group Theorem and the Closed Subgroup Theorem. J. Lie Theory 2008, 18, 383-390.

7. Hofmann, K.H.; Morris, S.A. The structure of almost connected pro-Lie groups. J. Lie Theory 2011, 21, 347-383.

8. Hofmann, K.H.; Morris, S.A. Local Splitting of Locally Compact Groups and Pro-Lie Groups. J. Group Theory 2011, 14, 931-935.

9. Hofmann, K.H.; Morris, S.A. The Structure of Compact Groups, 3rd ed., Revised and Augmented; Verlag Walter De Gruyter Berlin: Berlin, Germany, 2013.

10. Glöckner, H.; Neeb, K.-H. Infinite Dimensional Lie Groups, I, Basic Theory and Main Examples. 2015, in preparation.

11. Glöckner, H.; Neeb, K.-H. Infinite Dimensional Lie Groups, II, Geometry and Topology. 2015 , in preparation.

12. Neeb, K.-H. Towards a Lie theory of locally convex group. Japanese J. Math. 2006, 1, 291-468.

13. Hofmann, K.H.; Neeb, K.-H. Pro-Lie groups which are infinite dimensional Lie groups. Math. Proc. Cambridge Philos. Soc. 2008, 46, 351-378. 
14. Bogfjellmo, G.; Dahmen, R.; Schmeding, A. Character groups of Hopf algebras as infinite-dimensional Lie groups. arXiv:1501.05221v2 [math.GR], 2015.

15. Montgomery, D.; Zippin, L. Topological Transformation Groups; Interscience Publishers: New York, NY, USA, 1955.

16. Hofmann, K.H.; Morris, S.A. Contributions to the structure theory of connected pro-Lie groups. Topology Proc. 2008, 33, 225-237.

17. Oxtoby, J.C. Cartesian products of Baire spaces. Fundamenta Math. 1961, 49, 157-166.

18. Glöckner, H. Simplified Proofs for the Pro-Lie Group Theorem and the One-Parameter Subgroup Lifting Lemma. J. Lie Theory 2007, 17, 899-902.

19. Michael, G.A.A. On Inverse Limits of Finite-Dimensional Lie Groups. J. Lie Theory 2006, 16, 221-224.

20. Borel, A. Limites projectives de groupes de Lie. C.R. Acad. Sci. Paris 1950, 230, 1127-1128.

21. Ribes, L.; Zalesskii, P. Profinite Groups; Springer-Verlag Berlin: Heidelberg, Germany, 2000.

22. Wilson, J.S. Profinite Group; Oxford Science Publ.: Oxford, UK, 1998.

23. Roelcke, W.; Dierolf, S. Uniform Structures on Topological Groups and their Quotients; McGraw-Hill: New York, NY, USA, 1981.

24. Schaefer, H.; Wolff, M. Topological Vector Spaces; Springer: New York, NY, USA, 1999.

25. Banaszczyk, W. Additive Subgroups of Topological Vector Spaces; Springer-Verlag: Berlin, Germany, 1991.

26. Außenhöfer, L. The Lie Algebra of a Nuclear Group. J. Lie Theory 2003, 13, 263-270.

27. Hofmann, K.H.; Morris, S.A. An Open Mapping Theorem for pro-Lie Groups. J. Austral. Math. Soc. 2007, 83, 55-77.

28. Hofmann, K.H.; Morris, S.A. Open Mapping Theorems for Topological Groups. Topology Proc. 2007, 31, 533-551.

29. Hofmann, K.H.; Morris, S.A. Sophus Lie's Third Fundamental Theorem and the Adjoint Functor Theorem. J. Group Theory 2005, 8, 115-133.

30. Michael, G.A.A. The generalized Gluškov-Iwasawa local splitting theorem. J. Math. Sci. Univ. Tokyo 2010, 17, 123-133.

31. Hofmann, K.H.; Morris, S.A. Iwasaswa's local splitting theorem for Pro-Lie groups. Forum Mathematicum 2008, 20, 607-629.

32. Dahmen, R. Tensor Products of Weakly Complete Vector Spaces. Diplomarbeit am FB. Math. der Technischen Universität Darmstad 2007, 230, 66.

33. Michaelis, W. Coassociative Coalgebras; In Handbook of Algebra; Elsevier: Amsterdam, The Netherlands, 2003; pp. 587-788. 


\section{Non-Abelian Pseudocompact Groups}

\section{W. W. Comfort and Dieter Remus}

Abstract: Here are three recently-established theorems from the literature. (A) (2006) Every non-metrizable compact abelian group $K$ has $2^{|K|}$-many proper dense pseudocompact subgroups. (B) (2003) Every non-metrizable compact abelian group $K$ admits $2^{2^{|K|}}$-many strictly finer pseudocompact topological group refinements. (C) (2007) Every non-metrizable pseudocompact abelian group has a proper dense pseudocompact subgroup and a strictly finer pseudocompact topological group refinement. (Theorems (A), (B) and (C) become false if the non-metrizable hypothesis is omitted.) With a detailed view toward the relevant literature, the present authors ask: What happens to (A), (B), (C) and to similar known facts about pseudocompact abelian groups if the abelian hypothesis is omitted? Are the resulting statements true, false, true under certain natural additional hypotheses, etc.? Several new results responding in part to these questions are given, and several specific additional questions are posed.

Reprinted from Axioms. Cite as: Comfort, W.W.; Remus, D. Non-Abelian Pseudocompact Groups. Axioms 2016, 5, 2.

\section{Introduction}

Specific references to the literature concerning Theorems (A), (B) and (C) of the Abstract are given in 5.7(d), 8.2.2 and 4(l), respectively. Every metrizable pseudocompact group, abelian or not, is compact, hence admits neither a proper dense pseudocompact subgroup nor a proper pseudocompact group refinement (see 4(a)); thus, (A), (B) and (C) all become false when the non-metrizability hypothesis is omitted.

All hypothesized topological spaces and topological groups in this paper are assumed to be Tychonoff spaces.

\subsection{Brief Outline of the Paper}

As our Title and Abstract indicate, our goal in this survey is to describe the historical development of the theory of pseudocompact topological groups. Many of the results we cite, especially the older results, require an abelian hypothesis; some questions, definitions and results make sense and are correct without that hypothesis, however, and we emphasize these. Thus, this paper has two goals: (1) to provide an overview of the (by now substantial) literature on pseudocompact groups; and (2) to offer several new results about non-abelian pseudocompact groups. 
As an aid to the reader and to avoid uncertainty, algebraic statements and results known to hold also for non-abelian groups carry the symbol *.

We proceed as follows. Subsection 1.2 establishes the notation and terminology, and Subsection 1.3 reviews early works.

Section 2 describes several criteria, some algebraic and some cardinality-related, which are necessary or sufficient that a group admits a pseudocompact group topology. With a focus on compact groups, Section 3 describes the availability of proper dense subgroups. Section 4 recounts the principal incremental steps in the literature which led finally to a positive solution to these questions: Does every non-metrizable pseudocompact abelian group admit a proper dense subgroup and a strictly finer pseudocompact group topology? Section 5 considers briefly several miscellaneous issues and questions which concern pseudocompact groups.

Several workers have noted that those compact groups which admit a continuous epimorphism onto a product of the form $F^{\kappa}(|F|>1, \kappa>\omega)$ or of the form $\Pi_{i \in I} K_{i}\left(\left|K_{i}\right|>1,|I|>\omega\right)$ admit (sometimes large) families of dense subgroups with special properties. Section 6 describes several instances in the literature.

Sections 7 and 8 concern respectively free compact (abelian and non-abelian) groups over a Tychonoff space and new results concerning non-abelian pseudocompact groups.

Insofar as expository clarity permits, we use the symbol $K$ to denote a topological group known or assumed to be compact; and we use the symbol $G$ for other groups and topological groups.

\subsection{Notation and Terminology}

As to notation and terminology, we generally follow Engelking [1] and Hewitt and Ross [2]. Here we record some supplemental definitions, notation and conventions.

(a) Given a cardinal number $\alpha=\alpha_{0} \geq \omega$, the cardinal $\beth_{\omega}(\alpha)$ is defined as follows: $\alpha_{n+1}:=2^{\alpha_{n}}$ for $n<\omega$, and $\beth_{\omega}\left(\alpha_{0}\right):=\Sigma_{n} \alpha_{n}=\sup _{n} \alpha_{n}$.

(b) *For topological groups $G_{0}$ and $G_{1}$, we write $G_{0} \simeq G_{1}$ if some bijection from $G_{0}$ onto $G_{1}$ is simultaneously an algebraic isomorphism and a topological homeomorphism.

(c) *A topological group $G=(G, \mathcal{T})$ is totally bounded (alternatively, precompact) if for every non-empty $U \in \mathcal{T}$ there is finite $F \subseteq G$ such that $G=F U$. We denote by $\operatorname{TB}(G)$ the set of totally bounded group topologies on a group $G$.

(d) (Hewitt [3]) A space $X$ is pseudocompact if each continuous function $f$ : $X \rightarrow \mathbb{R}$ is bounded.

(e) A space is countably compact if each of its infinite subsets has an accumulation point (equivalently ([1]) if each countable open cover admits a finite subcover). 
(f) A space is $\omega$-bounded if each of its countably-infinite subsets has compact closure.

(g) A space $X$ is a Baire space if every intersection of countably many dense open subsets of $X$ is again dense in $X$.

(h) *A cardinal $\kappa$ is admissible if there is a pseudocompact group of cardinality $\kappa$. And $\kappa$ is $\lambda$-admissible if there is a pseudocompact group such that $|G|=\kappa$ and $w G=\lambda$.

(i) * Given a topological group $G$, we write

$\mathbb{P}(G):=\{H:|H|$ is a dense pseudocompact subgroup of $G\}$, and, following [4], for a compact group $K$ we write

$\mathfrak{m}(K):=\min \{|H|: H \in \mathbb{P}(K)\}$.

(j) *For a topological space $X=(X, \mathcal{T})$, we denote by $P X$, or by $(X, P \mathcal{T})$, the set $X$ with the smallest topology in which each $G_{\delta}$-subset of $(X, \mathcal{T})$ is open.

It is clear from the definitions of $P K$ and $\mathfrak{m}(K)$ that ${ }^{*} \mathfrak{m}(K)=d(P K)$; hence ${ }^{*} \operatorname{cf}(\mathfrak{m}(K))>\omega$, for each infinite compact group $K$.

(k) It is well known [2] (7.7) that a compact group $K$ is totally disconnected (equivalently: zero-dimensional) if and only if each neighborhood of $1_{K}$ contains a compact open normal subgroup. In this paper we follow many workers and call such compact groups $K$ profinite.

\subsection{Basic Early Works}

Here we offer a brief history of the principal concepts and objects we deal with in this paper.

(a) Hewitt [3] showed inter alia that a space $X$ is pseudocompact if and only if it is $G_{\delta}$-dense in its Stone-Čech compactification $\beta X$, hence in every (Tychonoff) space in which it is densely embedded. Later, Glicksberg [5] characterized pseudocompact spaces as those in which each locally finite family of open subsets is finite. For a detailed treatment and extrapolation of Hewitt's work, including the many other fruitful concepts introduced there, see [6].

(b) It is a fundamental theorem of Weil [7] that *the totally bounded groups are exactly the topological groups $G$ which embed as a dense topological subgroup of a compact group. Further, this compactification of $G$, called the Weil completion of $G$ and here denoted $\bar{G}$, is unique in the obvious sense.

(c) It is easy to see ([8] (1.1)) that *every pseudocompact group is totally bounded. Identifying those totally bounded groups which are pseudocompact, Comfort and Ross [8] $(1.2,4.1)$ showed that *for a totally bounded group $G$, these conditions are equivalent: (1) $G$ is pseudocompact; (2) $G$ is $G_{\delta}$-dense in $\bar{G}$; and (3) $\bar{G}=\beta G$. 


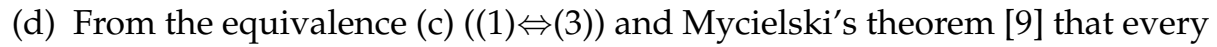
compact divisible group is connected, Wilcox [10] deduced a useful consequence: *every divisible pseudocompact group is connected (we note in passing, as remarked by Wilcox [10] (p. 579), that a connected pseudocompact abelian group need not be divisible).

(e) From (c) and the uniqueness aspect of Weil's theorem it follows that *a dense subgroup $H$ of a pseudocompact group $G$ is itself pseudocompact if and only if $H$ is $G_{\delta}$-dense in $G$ - in which case necessarily $\bar{H}=\bar{G}$; further, as in [8] (1.4), ${ }^{*}$ the product of any set of pseudocompact groups is again pseudocompact. Those two statements have been vastly generalized by subsequent workers. We give some examples. In (1) and (2), $G=\Pi_{i \in I} G_{i}$ with each $G_{i}$ an arbitrary (not necessarily abelian or pseudocompact) topological group, $F_{i} \subseteq G_{i}$ and $F:=\prod_{i \in I} F_{i}$. (1) [11] ${ }^{*}$ If $F_{i}$ is functionally bounded in $G_{i}$ in the sense that each continuous $f: G_{i} \rightarrow \mathbb{R}$ is bounded on $F_{i}$, then $F$ is functionally bounded in $G$; (2) [12] ${ }^{*}$ If $F_{i} \subseteq G_{i}$ is pseudocompact and either each $F_{i}$ is a $G_{\delta}$-set in $G_{i}$ or each $F_{i}$ is a retract of $G_{i}$, then $F$ is pseudocompact; (3) [13] ${ }^{*}$ If $K$ is compact and $X$ is $G_{\delta}$-dense in $K$, then $X$ is pseudocompact and $K=\beta X$; (4) [14] ${ }^{*}$ If $G$ is pseudocompact and $X$ is dense in $G$, then $X$ is $C$-embedded in $\operatorname{cl}_{P G} X$.

(f) The equivalences of (c) were established in [8] using earlier theorems of Kakutani and Kodaira [15], Halmos [16] (§64) and Ross and Stromberg [17]. A more direct approach, avoiding reference to those works, was given subsequently by de Vries [18]. See also Hušek [19] and Tkachenko [11,20] for alternative approaches.

(g) * Many of the results cited above have been extended and generalized into the context of locally pseudocompact groups; see, for example, [21,22] and the references given there.

\section{Pseudocompactifiability Criteria: Elementary Constraints}

(a) Every pseudocompact space is a Baire space [1] (3.10.F(e)), so in particular * every pseudocompact group is a Baire space (alternatively one may argue as in [4] (2.4(b)): a $G_{\delta}$-dense subspace of a Baire space is itself a Baire space, so a pseudocompact group $G$, being $G_{\delta}$-dense in the compact space $\bar{G}$, is necessarily a Baire space).

(b) Using (a), several workers (e.g., [4,23], [24] (2.5), [25]) made elementary cardinality observations like these, valid for infinite pseudocompact groups $G$. (1) ${ }^{*}|G| \geq \mathfrak{c} ;(2){ }^{*} d(P G) \geq \mathfrak{c} ;(3){ }^{*} \mathrm{cf}(d(P G))>\omega ;(4) *$ if $|G|$ is a strong limit cardinal, then $\operatorname{cf}(|G|)>\omega$; (5) if $G$ is abelian, then either $r_{0}(G) \geq \mathfrak{c}$ or $G$ is torsion; (6) if $G$ is a torsion abelian group, then $G$ is of bounded order.

Concerning (b): Van Douwen [23], arguing in a more general context, proved $|X| \geq \mathfrak{c}$ and other inequalities of cardinality type for every infinite pseudocompact space $X$ with no isolated point. 
(c) We remark in passing that the relation $\omega=\operatorname{cf}(|G|)$ does occur for some pseudocompact abelian groups in some models of ZFC . For example, if $\mathfrak{c}=\aleph_{1}<$ $\aleph_{\omega}<2^{\mathfrak{c}}$, then, as noted below in 3(e), the group $K=\mathbb{T}^{\mathfrak{c}}$ contains a proper dense countably compact subgroup $H$ with $|H|=\mathfrak{c}$, and then any group $G$ such that $H \subseteq G \subseteq K$, say with $|G|=\aleph_{\omega}$, is necessarily pseudocompact by $1.3(\mathrm{c})((2) \Rightarrow(1))$ (with $K=\bar{H}=\bar{G}$ ).

(d) The remarks in (b) are useful, but they are largely negative in flavor. Here are some simple examples. (1) *There is no countably infinite pseudocompact group; (2) *A compact group $K$ such that $w K=\beth_{\omega}(\alpha)$, satisfies $d K=\beth_{\omega}(\alpha)$; (3) ${ }^{*}$ If [CH] fails, no infinite pseudocompact group satisfies $|G|=\aleph_{1}$.

\section{Dense Subgroups: Scattered Results}

Some topological groups do, and some do not, have proper dense subgroups. Here we cite some representative results from the literature.

(a) ${ }^{*}$ The relations $d K \leq w K<2^{w K}=|K|$, valid for every infinite compact group $K([26](28.58(\mathrm{c})))$, make it clear that each such $K$ admits a (proper) dense subset $D$ with $|D|<|K|$, which then in turn generates a proper dense subgroup of the same cardinality. For emphasis: *every infinite compact group $K$ admits a proper dense subgroup $G$ with $|G|<|K|$. Similarly it follows easily, as in [4] (2.2(b)), that * for $K$ a compact group with $w(K)=\alpha \geq \omega$, one has $\mathfrak{m}(K) \leq(\log (\alpha))^{\omega}$.

(b) [4] (2.7(a)) * Infinite compact groups $K, K^{\prime}$ with $w(K)=w\left(K^{\prime}\right)$ satisfy $\mathfrak{m}(K)=\mathfrak{m}\left(K^{\prime}\right)$. Hence ${ }^{*} \mathfrak{m}(K)$ is determined fully by $w(K)$ and is not affected by algebraic properties of the group $K$. Following [4], we define $\mathfrak{m}(\alpha):=\mathfrak{m}(K)$ for (arbitrary) compact $K$ with $w(K)=\alpha$. Note: for $\alpha \geq \omega$, the cardinal $\mathfrak{m}\left(\{0,1\}^{\alpha}\right)$, which is $\mathfrak{m}(\alpha)$, is denoted $\Delta(\alpha, \omega)$ in [27].

(c) ([27]) If the Singular Cardinals Hypothesis is assumed (that is: $\kappa^{\lambda} \leq 2^{\lambda} \cdot \kappa^{+}$ for all infinite $\kappa, \lambda)$, then $\mathfrak{m}(\alpha)=(\log (\alpha))^{\omega}$.

(d) Every infinite pseudocompact group*, and every infinite connected abelian group, has a proper dense subgroup [28] $(4.1,4.2)$.

(e) For compact groups $K$ with $w(K)$ of the form $w(K)=2^{\alpha}$, it was shown by Itzkowitz [29] (the abelian case) and by Wilcox [30] in general that ${ }^{*} K$ contains a (necessarily proper) dense pseudocompact subgroup $H$ such that $|H| \leq \alpha^{\omega} \leq 2^{\alpha}<$ $2^{2^{\alpha}}=|K|$. It was noted later [31] that ${ }^{*} H$ may be chosen countably compact.

(f) Negating the tempting conjecture that parallel results might hold for locally compact groups, Rajagopalan and Soundrarajan [32] show that for each infinite cardinal $\kappa$ there is on the group $\mathbb{T}^{\kappa}$ a locally compact group topology which admits no proper dense subgroup. In the same vein, there are many infinite totally bounded abelian groups which admit no proper dense subgroup [28]. 
(g) In fact, given an abelian group $G$, the topology induced on $G$ by $\operatorname{Hom}(G, \mathbb{T})$ is a totally bounded group topology [33] (1.5) in which every subgroup is closed [31] (2.1).

\section{Extremal Phenomena}

We adopt terminology introduced tentatively and partially in [34] (5.1) and finally fully formalized in [35] (4.1): *a pseudocompact group $(G, \mathcal{T})$ is $r$-extremal (resp., s-extremal) if no pseudocompact group topology on $G$ strictly contains $\mathcal{T}$ (resp., $(G, \mathcal{T})$ admits no proper dense pseudocompact subgroup). Note: the letters $r$ and $s$ here are intended to invoke the words refinement and subgroup, respectively.

(a) Since a pseudocompact normal space is countably compact ([1] (3.10.21), [6] (3.D.2)) and a countably compact metric space is compact [1] (4.1.17), we have, as noted frequently in the literature ([36] (4.5(a)), [37] (3.1), [34] $(2.4,3.6))$ : *every pseudocompact group $G$ with $w(G) \leq \omega$ is both $r$ - and s-extremal. This explains the occurrence of the hypothesis " $w(G)>\omega$ " (equivalently: " $G$ is non-metrizable") in many of the theorems cited below.

It was conjectured in [34] (5.1ff.) that no non-metrizable pseudocompact abelian group is $r$ - or s-extremal (see also Question 2.B.1 in [38]). In the earliest days of investigation, the non-abelian case seemed totally inaccessible; but some fragmentary non-abelian results have emerged serendipitously by now (see below). Concerning the abelian question, the reader interested not in preliminary or incremental stages but only in the dénouement may safely ignore (b)-(k) below and skip directly to (l). For a more leisurely treatment of the historical development of this theorem, and for the statement of several related unsolved problems, see [39].

(b) A non-metrizable compact abelian group is not $r$-extremal [37] (3.4).

(c) A non-metrizable compact totally disconnected abelian group is neither $r$ nor s-extremal [36] $(4.3,4.4)$.

(d) A non-metrizable compact abelian group is neither $r$-nor $s$-extremal; indeed, the witnessing dense subgroup may be chosen $\omega$-bounded [34] (3.4).

(e) ${ }^{*}$ A non-metrizable compact connected group is not $r$-extremal [40] (6.7).

(f) A non-metrizable zero-dimensional pseudocompact abelian group is neither $r$ - nor s-extremal [34] (7.3).

(g) A pseudocompact abelian group $G$ such that $|G|>\mathfrak{c}$ or $\omega_{1} \leq w(G) \leq \mathfrak{c}$ is not s-extremal [41] (1.3).

(h) A pseudocompact abelian group $G$ such that $r_{0}(G)>\mathfrak{c}$ or $\omega_{1} \leq w(G) \leq \mathfrak{c}$ is not $r$-extremal [42] (5.10).

(i) A pseudocompact connected non-divisible abelian group is neither s-extremal [25] (7.1) nor $r$-extremal [43] (6.1), [42] (4.5(b)). 
(j) A pseudocompact abelian group $G$ with a closed $G_{\delta}$-subgroup $H$ (1) is $r$-extremal if $H$ is $r$-extremal, and (2) is s-extremal if $H$ is s-extremal ([42] (2.1)).

(k) If $H$ is a closed pseudocompact subgroup of a pseudocompact abelian group $G$, then (1) $G$ is not $r$-extremal if $G / H$ is not $r$-extremal, and (2) $G$ is not $s$-extremal if $G / H$ is not s-extremal [43] (4.5), [42] (5.3).

(l) (See (C) of the Abstract.) Fully familiar with the sources cited in (b)-(k), and drawing on some of the arguments cited there, Comfort and van Mill showed [44,45] that no non-metrizable abelian pseudocompact group is $r$-extremal or $s$-extremal.

As suggested above and as our title indicates, we are interested in the present paper primarily in comparable and parallel results concerning non-abelian pseudocompact groups. See in this connection especially Sections 6 and 8 .

\subsection{Extremality Questions}

As indicated in 4(1), the following two questions have been answered affirmatively $[44,45]$ in the context of abelian groups. However, they remain unsettled in the general (possibly non-abelian) case.

Problem 4.1.1. (a) * Is every non-metrizable pseudocompact group not $r$-extremal?

(b) ${ }^{*}$ Is every non-metrizable pseudocompact group not s-extremal?

(c) *Are those properties ( $r$ - extremal, s-extremal) equivalent?

\section{Related Concepts}

It was natural that workers thinking about the issues raised in Section 4 might be drawn simultaneously to different but related questions. Here, with no pretense to completeness, we mention some of these.

\subsection{Refinements of Maximal Weight}

When a pseudocompact group $G$ admits a proper pseudocompact refinement, can that be chosen of maximal weight (that is, of weight $2^{|G|}$ )? Comfort and Remus [40] (5.5) responded positively for many (non-metrizable) compact abelian groups $K$, including for example those which are connected, or torsion, or which satisfy $\operatorname{cf}(w(K))>w$. Later Comfort and Galindo [46] gave a positive answer for all non-metrizable compact abelian groups $G$ [46] (5.1), also for non-metrizable pseudocompact abelian groups $G$ which are torsion-free with $w G \leq|G|=|G|^{\omega}$ [46] (5.3) or (assuming [GCH]) which are torsion-free [46] (5.4(b)). Indeed ([46] (5.2)), in the compact abelian case $K$ with $w(K)=\alpha>\omega$, there are $2^{2^{2^{\alpha}}}$-many pseudocompact group refinements of weight $2^{\alpha}$. 


\subsection{The Poset of Pseudocompact Refinements}

Given a pseudocompact group $(G, \mathcal{T})$ with $w(G, \mathcal{T})=\alpha$, let $\operatorname{PS}(G, \mathcal{T})$ (respectively, $\operatorname{CPs}(G, \mathcal{T})$ ) be the partially-ordered set of group topologies $\mathcal{U}$ on $G$ such that $\mathcal{U} \supseteq \mathcal{T}$ and $\mathcal{U}$ is pseudocompact (respectively, $\mathcal{U}$ is pseudocompact and connected). For each cardinal number $\gamma$ set

$P_{s_{\gamma}}=\{\mathcal{U} \in P s(G, \mathcal{T}): w(G, \mathcal{U})=\gamma\}$ and

$C P_{s_{\gamma}}=\left\{\mathcal{U} \in C P_{s}(G, \mathcal{T}): w(G, \mathcal{U})=\gamma\right\}$

From [47] (3.11) it follows that each $\mathcal{U} \in C P s_{\gamma}(G, \mathcal{T})$ satisfies $\alpha \leq$ $w(G, \mathcal{U}) \leq 2^{|G|}$.

We have shown [40] (6.6):

Theorem 5.2.1. ${ }^{*}$ Let $K=(K, \mathcal{T})$ be a compact, connected group such that $w(K)=\alpha>\omega$, and let $A$ be the connected component of the center of $K$. Then:

(a) if $\omega<\beta<\alpha$, then $K$ admits a pseudocompact group topology $\mathcal{U}$ such that $\mathcal{U} \supseteq \mathcal{T}$, $\mathcal{U} \neq \mathcal{T}$, and $w(K, \mathcal{U})=\alpha+2^{2^{\beta}} ;$ and

(b) if $w(A)=\alpha$ or $\operatorname{cf}(\alpha)>\omega$, then $K$ admits a pseudocompact group topology $\mathcal{U}$ with $\mathcal{U} \supseteq \mathcal{T}, \mathcal{U} \neq \mathcal{T}$, and $w(K, \mathcal{U})=2^{2^{\alpha}}$.

As usual the (a) width, the (b) height and the (c) depth of a partially ordered set $P$ are defined to be the supremum of the cardinality of those subsets of $P$ which are respectively (a) an anti-chain, (b) well ordered, and (c) anti-well ordered. If there is an anti-chain $A \subseteq P$ such that $|A|=$ width $(P)$, then we say that width $(P)$ is assumed, and similarly for height $(P)$ and $\operatorname{depth}(P)$.

Comfort and Remus [47] (6.7) proved the following

Theorem 5.2.2. ${ }^{*}$ Let $(K, \mathcal{T})$ be a compact, connected group, such that $w(K, \mathcal{T})=\alpha$ with $\operatorname{cf}(\alpha)>\omega$, and let $\alpha \leq \gamma \leq 2^{|K|}$. Define $\bar{\gamma}=\min \left\{\gamma^{+}, 2^{|K|}\right\}$. Then:

(a) $\left|P_{S}(K, \mathcal{T})\right|=\left|C P_{S}(K, \mathcal{T})\right|=2^{2^{|K|}}$;

(b) $\left|\operatorname{Ps}_{\gamma}(K, \mathcal{T})\right|=\left|C P s_{\gamma}(K, \mathcal{T})\right|=2^{\gamma \cdot|K|}$;

(c) $\operatorname{width}\left(P_{S_{\gamma}}(K, \mathcal{T})\right)=\operatorname{width}\left(C P_{S_{\gamma}}(K, \mathcal{T})\right)=2^{\gamma \cdot|K|}$, and these widths are assumed;

(d) height $\left(\operatorname{Ps}_{\gamma}(K, \mathcal{T})\right)=\operatorname{height}\left(C P_{S_{\gamma}}(K, \mathcal{T})\right)=\bar{\gamma}$, and these heights are assumed; and

(e) $\operatorname{depth}\left(\operatorname{Ps}_{\gamma}(K, \mathcal{T})\right)=\operatorname{depth}\left(C P s_{\gamma}(K, \mathcal{T})\right)=\gamma$, and these depths are assumed

In the proof of Theorem 5.2.2 the main tools are Theorem 5.2.1 and the following theorem ([47] (6.4)).

Theorem 5.2.3. ${ }^{*}$ Let $\left(K, \mathcal{T}_{1}\right)$ be a totally bounded topological group such that $w\left(K, \mathcal{T}_{1}\right)=$ $\alpha_{1}>\omega$ and the Weil completion is connected. Then every totally bounded group topology $\mathcal{T}_{0}$ on $K$ such that $\mathcal{T}_{0} \subseteq \mathcal{T}_{1}$ and $w\left(K, \mathcal{T}_{0}\right)=\alpha_{0}<\alpha_{1}$ satisfies $\left|\left[\mathcal{T}_{0}, \mathcal{T}_{1}\right]\right|=2^{\alpha_{1}}$. 
In the absence of the connectivity hypothesis, we proved this result [48] (2.6(a)).

Theorem 5.2.4. * Let $G$ be a group, and let $\mathcal{T}_{i} \in \operatorname{TB}(G)(i=0,1)$ with $w\left(G, \mathcal{T}_{i}\right)=\alpha_{i} \geq \omega$. If $\alpha_{0}<\alpha_{1}$ and $\mathcal{T}_{0} \subseteq \mathcal{T}_{1}$, then $\left|\left[\mathcal{T}_{0}, \mathcal{T}_{1}\right]\right| \geq \alpha_{1}$.

Corollary 5.2.5. ${ }^{*}$ Let $(G, \mathcal{T})$ be a pseudocompact group with $w(G, \mathcal{T})=\alpha>|G|$. Then there are at least $\alpha$-many pseudocompact group topologies on $G$ which are coarser than $\mathcal{T}$.

Proof. Using the technique of the proof of [49] (2.9), we obtain $\mathcal{U} \in \operatorname{TB}(G)$ with $w(G, \mathcal{U}) \leq|G|<\alpha=w(G, \mathcal{T})$ such that $\mathcal{U} \subseteq \mathcal{T}$. Then $|[\mathcal{U}, \mathcal{T}]| \geq \alpha$ by Theorem 5.2.4, and $(G, \mathcal{V})$ is pseudocompact for each $\mathcal{V} \in[\mathcal{U}, \mathcal{T}]$ (since $(G, \mathcal{T}$ ) is pseudocompact).

In [47] (6.9), one finds

Problem 5.2.6. ${ }^{*}$ Let $G$ be a group, and let $\mathcal{T}_{i} \in \operatorname{TB}(G)(i=0,1)$ with $w\left(G, \mathcal{T}_{i}\right)=\alpha_{i} \geq \omega$. If $\alpha_{0}<\alpha_{1}$ and $\mathcal{T}_{0} \subseteq \mathcal{T}_{1}$, must $\left|\left[\mathcal{T}_{0}, \mathcal{T}_{1}\right]\right|=2^{\alpha_{1}}$ ?

We add the following

Problem 5.2.7. ${ }^{*}$ Let $(K, \mathcal{T})$ be a non-metrizable compact, connected group.

(a) Does $\mathcal{T}$ admit a proper (connected) pseudocompact refinement of maximal weight $2^{|K|}$ ?

(b) Are there $2^{2^{|K|}}$-many (connected) pseudocompact group topologies on $\mathrm{K}$ which are finer than $\mathcal{T}$ ?

We note in passing in connection with Problem 5.2.7, as remarked in [47] (pp. 277-278) and in contrast with [47] (6.11), that a pseudocompact refinement of a connected (abelian) pseudocompact group need not itself be connected. We note also that when the non-metrizability hypothesis is omitted in Problem 5.2.7, the resulting Questions (a) and (b) have negative answers. See in this connection 4(a) above.

\subsection{Totally Dense Subgroups}

As usual, a subgroup $D$ of a topological group $G$ is totally dense in $G$ if $D \cap H$ is dense in $H$ for every closed normal subgroup $H$ of $G$. Several workers have turned attention to the question of the existence of totally dense pseudocompact subgroups of a given (usually compact) group. Since this topic is a bit removed from our central focus here, for details in this direction we simply refer the reader to the relevant papers known to us: [50,51], [36] (5.3), [4] (5.8), [52-55]. We note explicitly that, building upon and extending results from her thesis [56], Giordano Bruno and Dikranjan [57] characterized those compact abelian groups with a proper totally dense pseudocompact subgroup as those with no closed torsion $G_{\delta}$-subgroup. 


\subsection{Concerning the Group Topologies $\operatorname{supTB}(G)$ and $\sup P S(G, \mathcal{T})$}

It is easily seen that *the supremum of any nonempty set of totally bounded group topologies on a fixed group $G$ is another such topology. In particular, then * each group $G$ which admits a totally bounded group topology admits the largest such topology. As noted [33] $(1.6,1.7)$ above, for abelian groups $G$ this is the topology induced on $G$ by $\operatorname{Hom}(G, \mathbb{T})$. It is shown in [31] (2.2) that when $G$ is infinite abelian, that topology on $G$ is never pseudocompact; that is obvious now, in view of the result cited above in $4(1)$.

Concerning that supremum, we record here a conjecture of Comfort and van Mill [58].

Conjecture 5.4.1. Let $G$ be an abelian group which admits a pseudocompact group topology. Then the supremum of the pseudocompact group topologies on $G$ coincides with the largest totally bounded group topology on $G$ (that is, the topology induced on $G$ by $\operatorname{Hom}(G, \mathbb{T})$ ).

Conjecture 5.4.1 was established in [58] for abelian groups $G$ which satisfy any of these (overlapping) conditions: (1) $G$ is torsion; (2) $|G| \leq 2^{\mathfrak{c}}$; (3) $r_{0}(G)=|G|=|G|^{\omega}$; (4) $|G|$ is a strong limit cardinal with $r_{0}(G)=|G|$; (5) some pseudocompact group topology $\mathcal{T}$ on $G$ satisfies $w(G, \mathcal{T}) \leq \mathfrak{c} ;(6) G$ admits a compact group topology. However, the conjecture remains unsettled in full generality.

While neither the present authors nor the authors of [58] attempted to find the optimal non-abelian version of the theorems and conjecture just given, we note that the most naive non-abelian analogue, namely that the supremum of all pseudocompact group topologies on a (possibly non-abelian) group $G$ which admits such a topology coincides with the largest totally bounded group topology, fails dramatically, even in the metrizable case. The following result is taken from [47].

Theorem 5.4.2. ${ }^{*}$ Let $K$ be a compact, connected Lie group with trivial center, and let $\mathcal{T}$ be the usual product topology on $K^{\omega}$. Then $\mathcal{T}$ is the only pseudocompact group topology on $K^{\omega}[47](7.4(a))$, but $\mathcal{T}$ admits $2^{2^{\mathfrak{c}}}$-many totally bounded finer group topologies [47] $(7.4(b))$.

\subsection{Additional Extremality Theorems}

The techniques used in the papers cited in Section 4 were adapted and extended by Giordano Bruno [56,59] to achieve parallel extremality results for pseudocompact abelian groups $G$ which are even $\alpha$-pseudocompact in the sense that $G$ meets every non-empty intersection of $\alpha$-many open subsets of $\bar{G}=\beta G$.

Prior to the appearance of $[44,45]$, researchers in Udine, Italy, considered conditions weaker than metrizability which suffice to guarantee that a pseudocompact abelian group $G$ is both $r$ - and $s$-extremal [56,57,60,61]. Here is a sample result. 
Theorem 5.5.1. If some closed $G_{\delta}$-subgroup $N$ of $G$ admits a dense pseudocompact subgroup $H$ such that $r_{0}(N / H) \geq \mathfrak{c}$, then $G$ itself has such a subgroup (hence is neither $r$ nor s-extremal).

\subsection{Closed Subgroups of Pseudocompact Groups}

Since every subgroup of a totally bounded group is totally bounded and every closed subspace of a countably compact space is countably compact, it is reasonable (though perhaps naive) to inquire whether every closed subgroup of a pseudocompact group is necessarily pseudocompact. To the authors' knowledge, this question was first addressed in [31] (2.4), where a straightforward abelian counterexample is offered. Later this fact was noted (see [62] (2.1), [63] (2.9)):

Theorem 5.6.1. *Every totally bounded group $H$ embeds as a closed subgroup of a pseudocompact group $\mathrm{G}$. If $H$ is abelian, $G$ may be chosen as abelian.

The construction of [62] (2.1) shows, though the authors did not record the fact explicitly, that, ${ }^{*}$ when $H$ as in Theorem 5.6.1 is non-metrizable (that is, when $w(H)>\omega)$, one may choose $G$ so that $w(G)=w(H)$.

In the abelian case, the correct locally bounded analogue of Theorem 5.6.1 has been recorded by Ursul [64]:

Theorem 5.6.2. Every locally bounded abelian group is a closed subgroup of a locally pseudocompact group.

More recently, Leiderman, Morris and Tkachenko [65] have focused on closed embeddings into pseudocompact groups of small density character. For example, they have shown this.

Theorem 5.6.3. *Every totally bounded group $H$ such that $w(H) \leq \mathfrak{c}$ embeds as a closed subgroup of a separable pseudocompact group.

Since there are many totally bounded non-separable pseudocompact groups of weight $\mathfrak{c}$, for example the $\omega$-bounded group $H:=\left\{x \in \mathbb{T}^{\mathfrak{c}}:\left|\left\{\eta<\mathfrak{c}: x_{\eta} \neq 1_{\mathbb{T}}\right\}\right| \leq\right.$ $\omega\}$, it follows from Theorem 5.6.3, as is remarked in [65], that a closed subgroup of a separable pseudocompact abelian group can be non-separable.

\subsection{Miscellaneous Investigations}

(a) *Dikranjan and Shakhmatov [66] (3.7) extended an important result of $\mathrm{Zel}^{\prime}$ manov [67] (the compact case) to prove: every pseudocompact torsion group is locally finite.

(b) *The same authors investigated the following problem: Which infinite groups admit a pseudocompact group topology? We restrict attention here to non-abelian groups. A variety $\mathcal{V}$ of groups is said to be precompact if each $\mathcal{V}$-free 
group admits a precompact (totally bounded) group topology. An example is the variety of all groups. Here, we quote verbatim from [66] (1.3) * "a free group $F$ in a variety $\mathcal{V}$ admits a non-discrete pseudocompact group topology if and only if $\mathcal{V}$ is precompact and $|F|$ is admissible". For further results see [66] (Chapter 5).

(c) Dikranjan [68] proved (among other interesting theorems concerning pseudocompact abelian groups) this statement: ${ }^{*}$ Let $F$ be a free group in a variety, and let $\alpha \geq \omega$. If $|F|$ is $\alpha$-admissible, then the poset of all pseudocompact group topologies of weight $\alpha$ on $F$ contains a copy of the power set of $\alpha$.

(d) (See (A) of the Abstract.) *Comfort, Raczkowski and Trigos-Arrieta noted [69] (3.1) that in a compact group, every proper, $G_{\delta}$-dense subgroup (that is, every proper dense pseudocompact subgroup) is non-measurable (in the sense of Haar). They showed that every infinite abelian group $K$ of uncountable weight has $2^{|K|}$-many dense pseudocompact subgroups of cardinality $|K|[69]$ (3.2); hence such $K$ admits $2^{|K|}$-many dense non-measurable subgroups of cardinality $|K|$ [69] (3.4). In the same vein, Itzkowitz [70] (2.1) showed that every non-metrizable product-like group, defined as in Section 6.1, satisfies $|\mathbb{P}(K)|=2^{|K|}$ (the witnessing elements of $\mathbb{P}(K)$ being necessarily non-measurable). For further related results see [70].

Problem 5.7.1. Let $K$ be a non-metrizable compact group. Does $|\mathbb{P}(K)|=2^{|K|}$ hold?

By [71] (3.4) a strongly complete group is a profinite group in which every finite index subgroup is open. An infinite group is almost perfect if $\left|G / G^{\prime}\right|$ is finite for the algebraic commutator subgroup $G^{\prime}$ of $G$. Hernández, Hofmann and Morris [71] (3.5) proved: an infinite group in which every subgroup is measurable is a strongly complete almost perfect group. More recently Brian and Mislove [72] showed that it is consistent with ZFC that every infinite compact group has a non-measurable subgroup.

Problem 5.7.2. Does every infinite compact group $K$ have $\left.2^{|K|}\right|_{-m a n y}$ non-measurable subgroups (of cardinality $|K|$ )?

\section{Epimorphisms onto Products}

Comfort and Robertson [37] (3.2(b)) showed that each non-metrizable compact abelian group $K$ maps by a continuous epimorphism onto a group of the form $M^{\left(\omega^{+}\right)}$ with $M$ a compact subgroup of $\mathbb{T}$. From this they determined [37] (3.4) that such $K$ is not $r$-extremal.

\subsection{Product-Like Groups}

According to a definition of Itzkowitz and Shakhmatov ([70,73-75]) *a compact group $K$ with $\kappa=w(K)$ is product-like if there is a continuous epimorphism $h: K \rightarrow$ $\Pi_{\tilde{\zeta}<\kappa} M_{\xi}$ with each $M_{\xi}$ a non-trivial (compact) metrizable group. A similar class 
was introduced by Varopoulos [76] (§3): a compact group $K$ is called a $\prod$-group if $K \simeq \Pi_{i \in I} M_{i}$, where all $M_{i}$ are (compact) metrizable groups. Not every $\prod$-group $K$ is product-like: take for example for $K$ an algebraically simple compact group. In [76] it is proved that if $K$ is a connected, compact group with center $Z$, then $K / Z$ is a $\prod$-group. Comfort and Robertson [4] (4.2) showed that (for non-trivial $K$ ) the group $K / Z(K)$ is a product of compact, connected, non-abelian Lie groups which are algebraically simple.

It is known that every non-metrizable compact group $K$ which is either abelian or connected is product-like (see for example [40] (5.4) or [74] (1.11) for the abelian case, [40] (proof of 6.5) or [73] for the connected case). This allowed the authors of $[70,73-75]$ to conclude that for every such group $K$ the set $\Omega(K)$ of dense $\omega$-bounded subgroups of $K$ satisfies $|\Omega(K)| \geq|K|$. They asked whether that inequality may be improved to $|\Omega(K)|=2^{|K|}$; the question was answered affirmatively in [77] for those $K$ which in addition satisfy $w(K)=(w(K))^{\omega}$.

In this connection this question, raised in [77], appears still to be unsettled:

Problem 6.1.1. ${ }^{*}$ Does $|\Omega(K)|=2^{|K|}$ hold for every non-metrizable compact group $K$ ? What if $K$ is product-like? What if $\operatorname{cf}(w K)>\omega$ ?

*Itzkowitz [70] (p. 23) cites from [74,75] the statement that all non-metrizable compact groups which are connected or abelian are product-like. In the proof for connected groups ([75] (5)) the authors used [4] ((4.2), (4.3)). The paper [40] was cited in [74] (2.5), but not in [75]. The authors of [70,74,75] inadvertently failed to note that already in [40] (proof of (6.5)) the following more detailed result had been obtained in a more direct way (see also [40] (5.4) for a relevant result in the abelian context):

Lemma 6.1.2. * Let $K$ be an infinite compact, connected group, and let $A$ be the connected component of the center of $K$. Then:

(a) if $w(A)=w(K)$, then there is a continuous epimorphism $h: K \rightarrow \mathbb{T}^{w(K)}$; and

(b) if $w(A)<w(K)$, then there is a continuous epimorphism $h: K \rightarrow \Pi_{i \in I}\left(H_{i} / C_{i}\right)$ with $|I|=w(K)$.

In the statement of Lemma 6.1.2(b), each $H_{i}$ is a compact, simply connected, simple Lie group with finite center $C_{i}$. By a result of van der Waerden [78] all groups $H_{i} / C_{i}$ are algebraically simple.

Lemma 6.1.2 is fundamental for the following theorem, which is a main tool in the proof of Theorem 5.2.1.

Theorem 6.1.3 ([40] (6.5)). * Let $K$ be a compact, connected group such that $w(K)=\alpha>\omega$, and let $A$ be the connected component of the center of $K$. Then:

(a) if $\omega<\beta<\alpha$, then there are a compact group $F$ with $|F|>1$ and a continuous epimorphism from $\mathrm{K}$ onto $\mathrm{F}^{\beta}$; and 
(b) if $w(A)=\alpha$ or $\operatorname{cf}(\alpha)>\omega$, then there are a compact group $F$ with $|F|>1$ and $a$ continuous epimorphism from $K$ onto $F^{\alpha}$.

The proof of Theorem 5.2.1 depends crucially on the following result.

Theorem 6.1.4 ([40] (5.2)). ${ }^{*}$ Let $\kappa>\omega$, let $K=(K, \mathcal{T})$ be a compact group, and let $h: K \rightarrow F^{\mathcal{K}}$ be a continuous epimorphism with $F$ a compact group, $|F|>1$. Then $K$ admits a pseudocompact group topology $\mathcal{U}$ such that $\mathcal{U} \supseteq \mathcal{T}, \mathcal{U} \neq \mathcal{T}$, and $w(K, \mathcal{U})=w(K, \mathcal{T})+2^{2^{\kappa}}$.

Remarks 6.1.5. (a) By no means is every compact group product-like. It is shown in [34] (4.10(d)) that * for every cardinal $\kappa \geq \omega$ there is a compact (non-abelian) group $K$ with $w K=\kappa$ such that no homomorphism $h: K \rightarrow H_{0} \times H_{1}$ with $\left|H_{i}\right|>1$ is surjective.

(b) It is shown in [46] (6.2) that when $\alpha:=\beth_{\omega}\left(\alpha_{0}\right)\left(\alpha_{0} \geq \omega\right)$, then with $K:=$ $\Pi_{n<\omega}\left(\mathbb{Z}\left(p_{n}\right)\right)^{\alpha_{n}}$ we have: every continuous surjective epimorphism $h: K \rightarrow F^{\mathcal{K}}$ with $|F|>1$ satisfies $\kappa<\alpha=w(K)$ (indeed $2^{2^{\kappa}}<2^{2^{\alpha}}$ ). Seeking a non-abelian result with a similar flavor, we have formulated (but not proved) several reasonable conjectures. Of these, Conjecture 6.1 .6 below seems particularly attractive and accessible. Here we say as usual that a topological group $K$ is topologically simple if the only closed normal subgroups of $K$ are $\left\{1_{K}\right\}$ and $K$ itself, and we recall this characterization of compact topologically simple groups, due to $\mathrm{Yu}$ [79] (1.8).

Every compact topologically simple group is either a finite simple group or a compact, algebraically simple, connected Lie group.

In particular, then, each such group $K$ is metrizable, i.e., satisfies $w K \leq \omega$.

We recall also, for example from [80] (7.3.11), that for $K$ as hypothesized below, every closed normal subgroup $N$ of $K$ (in particular the subgroup $N=\operatorname{ker}(h)$ ), has the form $N=\Pi_{n \in I} K_{n}^{\beta_{n}} \times\left\{1_{\omega \backslash I}\right\}$ for suitable $I \subseteq \omega$ and for suitable cardinals $\beta_{n} \leq \alpha_{n}$.

Conjecture 6.1.6. ${ }^{*}$ Let $\left(\alpha_{n}\right)_{n<\omega}$ be a strictly increasing sequence of infinite cardinals with each $\operatorname{cf}\left(\alpha_{n}\right)>\omega$, and set $\alpha:=\sup _{n} \alpha_{n}=\Sigma_{n} \alpha_{n}$. Let $\left\{K_{n}: n<\omega\right\}$ be a sequence of pairwise non-isomorphic, non-abelian, topologically simple compact groups, and set $K:=$ $\Pi_{n} K_{n}^{\alpha_{n}}$. Then:

(a) every continuous epimorphism $h: K \rightarrow F^{\mathcal{K}}$ with $|F|>1$ satisfies $\kappa<\alpha=w K$; and

(b) if $\alpha_{n+1}=2^{\alpha_{n}}$ for all $n$, so that $\alpha=\beth_{\omega}\left(\alpha_{0}\right)$, then $\kappa^{\beta}<w K$ for all $\beta<\alpha$.

Of course (b) follows from (a), since for such $\kappa$ and $\beta$ there is $n<\omega$ such that $\kappa<\alpha_{n}$ and $\beta<\alpha_{n}$, and then $\kappa^{\beta} \leq \alpha_{n}^{\alpha_{n}}=2^{\alpha_{n}}=\alpha_{n+1}<\alpha$. 


\section{Concerning Free Compact Topological Groups}

\subsection{Characterizations of FX and FAX}

Here we follow generally the conventions of Hofmann and Morris [81] (Chapter 11). See also [2] (8.8) for a less extensive, more constructive approach to free topological groups.

(a) For every space $X$ there is a compact group $F X$, the free compact group on $X$, such that

(1) $X \subseteq\langle X\rangle \subseteq F X$ with $X$ closed in $\langle X\rangle$ and $\langle X\rangle$ dense in $F X$;

(2) algebraically, $\langle X\rangle$ is the free group on the set $X$; and

(3) for every continuous $f: X \rightarrow K$ with $K$ a compact group there is a (unique) continuous homomorphism $\bar{f}: F X \rightarrow K$ such that $\bar{f} \mid X=f$.

The free compact abelian group FAX has analogous properties, with $\bar{f} \supseteq f$ : $X \rightarrow K$ with $K$ a compact abelian group.

(b) The role of $1_{F X}$ is played in $F X$ by the empty word. In contrast, some workers prefer to work with pointed spaces $(X, p)$, then with the identification $p \rightarrow 1 \cdot p=1_{F X} \in\langle X\rangle \subseteq F X$.

The theorem cited in (a) is rooted in the work of Markov [82,83] and Graev [84,85] concerning free topological groups. Alternate latter-day constructions abound, some achieved independently of [82-85] and some based on those works, some with algebraic emphasis [86,87], [2] (8.8,8.9), [24] (2.3-2.5), some topological [88-90], some functorial or categorical [91]. See [92] (\$4) for a comprehensive introduction to the groups $F X$ and $F A X$, and see [62] for generalizations to "free $\mathbb{P}$-spaces" for some other classes $\mathbb{P}$.

The reader will note that in our present convention, the "free compact group $F X^{\prime \prime}$ is a compact group which is not algebraically the free group on $X$. In the sources about free topological groups cited above, that is reversed: "the free topological group over a space $X^{\prime \prime}$ is itself not compact, it is algebraically the free group on $X$. Note that FX is the Bohr compactification (see [93] (Chapter 5.4)) of the free topological group over the space $X$.

\subsection{Basic Properties of FX and FAX}

(a) We list four basic facts about the free groups FX and FAX.

(i) ([92] (4.2.2)) *For each space $X$ the free compact group $F X$ is naturally isomorphic to the free compact group $F \beta X$, where $\beta X$ is the Stone-Čech compactification of $X$.

(ii) ${ }^{*} F X$ is connected if and only if $X$ is connected; similarly for FAX. 
(iii) ([81] (1.4), [92] (4.2.4)) *With $X$ given and with $F X$ in hand, the group $F A X$ may be "realized" in concrete form by the rule $F A X=F X / \overline{\left.(F X)^{\prime}\right)}$, with $(F X)^{\prime}$ denoting the commutator subgroup of $F X$;

(iv) ([92] (4.2.1(i), [81] (11.6)) *For $X$ compact and infinite one has $w(F X)=$ $w(F A X)=(w X)^{\omega}$; hence $\operatorname{cf}(w(F X))=\operatorname{cf}(w(F A X))>\omega$.

It follows from (ii), (iii) and (iv) that the free compact groups $F X$ and $F A X$ for $X$ compact and connected satisfy the conditions of Theorem 5.2.1(b) of Section 5. By applying Theorem 8.2.3 of Section 8 below we have this result.

Theorem 7.2.1. ${ }^{*}$ Let $F X=(F X, \mathcal{T})$ be the free compact group over the compact space $X$ with $|X|>1$ and $w(F X)=w(F A X)=\alpha>\omega$. Then with $\kappa:=2^{2^{2^{\alpha}}}$ there are $\kappa$-many pseudocompact group topologies $\mathcal{U}_{\eta}(\eta<\kappa)$ on FX such that $\mathcal{U}_{\eta} \supseteq \mathcal{T}, \mathcal{U}_{\eta} \neq \mathcal{T}$, and $w\left(F X, \mathcal{U}_{\eta}\right)=2^{|F X|}$.

\section{New Results, Non-Abelian Emphasis}

\subsection{Three Preliminary Lemmas}

It is shown in [46] (5.1) that every non-metrizable compact abelian group $K$ admits a pseudocompact group refinement of maximal weight (that is, of weight $2^{|K|}$ ). With a view toward generalizing that statement and its corollaries from [46] into the non-abelian context, we begin this section (making no claim for novelty in either case) with two simple lemmas.

Lemma 8.1.1. ${ }^{*}$ Let $H$ be a closed normal subgroup of an infinite totally bounded group $G$. Then $w G=w H+w(G / H)$.

Proof. The result is well known (see for example [40] (6.1)) when $G$, hence also $H$, is compact. Denoting as in $1.3($ b) by $\bar{G}$ the Weil completion of (an arbitrary) totally bounded group $G$, we have in the present case $\overline{G / H}=\bar{G} / \bar{H}$ (see also in this connection [93] (5.4.3) and [94] (2.6)), and hence

$$
w G=w \bar{G}=w \bar{H}+w(\bar{G} / \bar{H})=w H+w(G / H) .
$$

Lemma 8.1.2. [47] (3.11) * Let $\mathcal{S}$ and $\mathcal{U}$ be totally bounded group topologies on a group $G$ such that $\mathcal{S} \supseteq \mathcal{U}$. Then $w(G, \mathcal{S}) \geq w(G, \mathcal{U})$.

Proof. The continuous map id : $(G, \mathcal{S}) \rightarrow(G, \mathcal{U})$ extends continuously to id : $\overline{(G, \mathcal{S})} \rightarrow \overline{(G, \mathcal{U})}$, and a continuous surjection between compact spaces cannot raise weight [1] (3.1.22), so we have

$$
w(G, \mathcal{S})=w \overline{(G, \mathcal{S})} \geq w \overline{(G, \mathcal{U})}=w(G, \mathcal{U}) .
$$

Now for an arbitrary totally bounded group $(G, \mathcal{T})$ we denote by $\mathcal{M}(\mathcal{T})$ the set of totally bounded group topologies on $G$ which contain $\mathcal{T}$. Further, given a 
closed normal subgroup $H$ of $G$, we denote by $\phi$ the usual quotient map from $G$ onto $G / H$ and by $\mathcal{T}_{q}$ the quotient topology on $G / H$. We note that for a group topology $\mathcal{S}$ on $G / H$, the initial topology $\phi^{-1}(\mathcal{S})$ induced on $G$ by $\phi$ and $\mathcal{S}$ is a group topology which is not in general a Hausdorff topology.

Lemma 8.1.3. ${ }^{*}$ Let $K=(K, \mathcal{T})$ be a compact group with a closed normal subgroup $H$. Then:

(a) $\mathcal{S} \in \mathcal{M}\left(\mathcal{T}_{q}\right) \Rightarrow \mathcal{T} \vee \phi^{-1}(\mathcal{S}) \in \mathcal{M}(\mathcal{T})$

(b) the map $\mathcal{M}\left(\mathcal{T}_{q}\right) \rightarrow \mathcal{M}(\mathcal{T})$ given in (a) is injective; and

(c) if $\mathcal{S} \in \mathcal{M}\left(\mathcal{T}_{q}\right)$ is pseudocompact, then $\mathcal{T} \vee \phi^{-1}(\mathcal{S})$ is pseudocompact.

Proof. With only notational changes, the proof follows the argument of our work with Szambien [95] (3.5).

\subsection{Refinements of Large Weight (the Non-abelian Case)}

The following theorem is a generalization of [40] (6.2).

Theorem 8.2.1. ${ }^{*}$ Let $K=(K, \mathcal{T})$ be a compact group such that $w(K)=\alpha>\omega$. Let $K^{\prime}$ be the closure in $(K, \mathcal{T})$ of the commutator subgroup of $K$. If $w\left(K / K^{\prime}, \mathcal{T}_{q}\right)=\beta>\omega$, then there is a pseudocompact group topology $\mathcal{U}$ on $K$ such that $\mathcal{U} \supseteq \mathcal{T}, \mathcal{U} \neq \mathcal{T}$, and $w(K, \mathcal{U})=\alpha+2^{2^{\beta}}$.

Proof. From [46] (5.1) applied to the compact group $\left(K / K^{\prime}, \mathcal{T}_{q}\right)$ we have: there is a pseudocompact group topology $\mathcal{S} \in \mathcal{M}\left(\mathcal{T}_{q}\right)$ such that $\mathcal{S} \neq \mathcal{T}_{q}$ and $w\left(K / K^{\prime}, \mathcal{S}\right)=2^{2^{\beta}}$. The topology $\mathcal{U}:=\mathcal{T} \vee \phi^{-1}(\mathcal{S})$ on $K$ is pseudocompact by Lemma 8.1.3(c).

Lemma 8.1.1 implies $w(K, \mathcal{U})=w\left(K^{\prime}, \mathcal{U}_{0}\right)+w\left(K / K^{\prime}, \mathcal{S}\right)$, because $\mathcal{S}$ is the quotient topology of $\mathcal{U}$; here $\mathcal{U}_{0}$ denotes the topology induced by $\mathcal{U}$ on $K^{\prime}$. This topology coincides with the topology induced by $\mathcal{T}$ on $K^{\prime}$, so $w(K, \mathcal{U}) \leq \alpha+2^{2^{\beta}}$.

For the reverse equality we note that $w(K, \mathcal{U}) \geq w\left(K / K^{\prime}, \mathcal{S}\right)=2^{2^{\beta}}$. Lemma 8.1.2 gives $w(K, \mathcal{U}) \geq \alpha$. Hence $w(K, \mathcal{U}) \geq \alpha+2^{2^{\beta}}$.

In preparation for Theorem 8.2.3 we recall this result from [46] (5.2) (see (B) of the Abstract).

Lemma 8.2.2. For every compact abelian group $K=(K, \mathcal{T})$ with $w K=\alpha>\omega$ there are $2^{2^{|K|}}$-many pseudocompact group topologies $\mathcal{U}$ on $K$ such that $\mathcal{U} \supseteq \mathcal{T}, \mathcal{U} \neq \mathcal{T}$, and $w(K, \mathcal{U})=2^{|K|}$.

Theorem 8.2.3. ${ }^{*}$ Let $K=(K, \mathcal{T})$ be a compact group such that $w(K, \mathcal{T})=w\left(K / K^{\prime}, \mathcal{T}_{q}\right)=$ $\alpha>\omega$. Then with $\kappa:=2^{2^{2^{\alpha}}}$ there are $\kappa$-many pseudocompact group topologies $\mathcal{U}_{\eta}(\eta<\kappa)$ on $K$ such that $\mathcal{U}_{\eta} \supseteq \mathcal{T}, \mathcal{U}_{\eta} \neq \mathcal{T}$, and $w\left(K, \mathcal{U}_{\eta}\right)=2^{|K|}$.

Proof. In view of Lemma 8.1.1 and Lemma 8.1.3, it is enough to know that there are $\kappa$-many pseudocompact group topologies $\mathcal{S}_{\eta}(\eta<\kappa)$ on the abelian group $K / K^{\prime}$ such that $\mathcal{S}_{\eta} \supseteq \mathcal{T}_{q}, \mathcal{S}_{\eta} \neq \mathcal{T}_{q}$, and $w\left(K / K^{\prime}, \mathcal{S}_{\eta}\right)=2^{\left|K / K^{\prime}\right|}=2^{|K|}$. This is given by Lemma 8.2.2 (with $K$ there replaced by $K / K^{\prime}$ here). 
Remark 8.2.4. We adopt this definition from [81] (9.92). Given a group $G$, the set $F$ of all elements whose conjugacy class is finite is called the FC-center of $G$. If $F=G$, then $G$ is an FC-group. Hofmann and Morris [81] (9.99) proved this theorem:

* Let $K$ be a compact group. Then the following three statements are equivalent:

(i) $K$ is an FC-group;

(ii) $K / Z(K)$ is finite; and

(iii) the commutator subgroup of $K$ is finite.

From the implication (i) $\Rightarrow$ (iii) it is clear that every FC-group $K=(K, \mathcal{T})$ with $w(K)=\alpha>\omega$ satisfies the condition $w(K)=w\left(K / K^{\prime}\right)=\alpha$, so from Theorem 8.2.3 we have this consequence for such $K$ : with $\kappa:=2^{2^{2^{\alpha}}}$ there are $\kappa$-many pseudocompact group topologies $\mathcal{U}_{\eta}(\eta<\kappa)$ on $K$ such that $\mathcal{U}_{\eta} \supseteq \mathcal{T}, \mathcal{U}_{\eta} \neq \mathcal{T}$, and $w\left(K, \mathcal{U}_{\eta}\right)=2^{|K|}$.

Theorem 8.2.5. ${ }^{*}$ Let $K=(K, \mathcal{T})$ be a compact group, such that $w(K)=\alpha>\omega$, and let $Z_{0}(K)$ be the connected component of the center $Z(K)$ of $K$. If $w\left(Z_{0}(K)\right)=\beta>\omega$, then there is a pseudocompact group topology $\mathcal{U}$ on $K$ such that $\mathcal{U} \supseteq \mathcal{T}, \mathcal{U} \neq \mathcal{T}$, and $w(K, \mathcal{U}) \geq \alpha+2^{2^{\beta}}$.

Proof. Let $K^{\prime}$ be the closure of the commutator subgroup of $K$. By [81] (9.23(iii)) the connected component $C_{0}$ of $K / K^{\prime}$ is topologically isomorphic to $Z_{0}(K) / H$, where $H$ is the intersection of $Z_{0}(K)$ and $K^{\prime}$. The group $H$ is totally disconnected, since $Z(K) \cap K^{\prime}$ is totally disconnected by [81] (9.23(i)). Thus [96] (3.2) implies $w\left(Z_{0}(K)\right)=w\left(C_{0}\right)$. Hence $\beta \leq w\left(K / K^{\prime}\right)$. Now apply Theorem 8.2.1 to complete the proof.

Let $\mathcal{R}$ denote a set of representatives for the isomorphism classes of the class of all compact simple groups. It was noted by Hofmann and Morris [97] (p. 412) that $\mathcal{R}$ is an infinite countable set. In [97] (2.2), a compact group is called strictly reductive if it is isomorphic to a Cartesian product of compact algebraically simple groups. For a compact group $K$ and $S \in \mathcal{R}$, the smallest closed subgroup $K_{S}$ of $K$ containing all closed normal subgroups isomorphic to $S$ is called in [97] the S-socle of $K$.

Theorem 8.2.6 ([97] (2.3)). * Let $K$ be a strictly reductive compact group, and let $\left(K_{S}\right)_{S \in \mathcal{R}}$ denote the sequence of $S$-socles of $K$. Then there is a sequence of cardinals $(J(K, S))_{S \in \mathcal{R}}$ such that $K \simeq \Pi_{S \in \mathcal{R}} K_{S}$, with $K_{S} \simeq S^{J(K, S)}$ for each $S \in \mathcal{R}$.

Theorem 8.2.7. ${ }^{*}$ Let $(K, \mathcal{T})$ be a strictly reductive compact group with $w(K, \mathcal{T})=\alpha>\omega$. Then:

(a) if $\omega<\beta<\alpha$, then $K$ admits a pseudocompact group topology $\mathcal{U}$ such that $\mathcal{U} \supseteq \mathcal{T}$, $\mathcal{U} \neq \mathcal{T}$, and $w(K, \mathcal{U})=\alpha+2^{2^{\beta}}$; and

(b) if $\operatorname{cf}(\alpha)>\omega$, then $K$ admits a pseudocompact group topology $\mathcal{U}$ with $\mathcal{U} \supseteq \mathcal{T}$, $\mathcal{U} \neq \mathcal{T}$, and $w(K, \mathcal{U})=2^{2^{\alpha}}$.

Proof. By Theorem 8.2.6 there is a sequence of cardinals $(J(K, S))_{S \in \mathcal{R}}$ such that $K \simeq \Pi_{S \in \mathcal{R}} K_{S}, K_{S} \simeq S^{J(K, S)}$. Hofmann and Morris [97](2.7) showed $w(K, \mathcal{T})=$ $\max \{\omega, \sup \{J(K, S): S \in \mathcal{R}\}\}$. Then Theorem 6.1.4 completes the proof. 
Corollary 8.2.8. ${ }^{*}$ Let $(K, \mathcal{T})$ be a strictly reductive compact group with $w(K, \mathcal{T})=\alpha>\omega$. Then $\mathrm{K}$ admits a pseudocompact group topology $\mathcal{U}$ with $\mathcal{U} \supseteq \mathcal{T}, \mathcal{U} \neq \mathcal{T}$.

Proof. If $\alpha>\omega^{+}$, use Theorem 8.2.7(a). If $\alpha=\omega^{+}$, use Theorem 8.2.7(b).

Corollary 8.2.9. ${ }^{*}$ Let $(K, \mathcal{T})$ be a strictly reductive compact group with $w(K, \mathcal{T})=\alpha$. If $\operatorname{cf}(\alpha)>\omega$, then $K$ admits a pseudocompact group topology $\mathcal{U}$ with $\mathcal{U} \supseteq \mathcal{T}, \mathcal{U} \neq \mathcal{T}$, and $|[\mathcal{T}, \mathcal{U}]| \geq 2^{|K|}$.

Proof. Use Theorem 5.2.4 and Theorem 8.2.7(b).

Remus considered in [98] uncountable powers of a non-abelian, compact, topologically simple group.

Theorem 8.2.10 ([98] (3.40)). * Let F be a non-abelian, compact, topologically simple group, and let $\alpha>\omega$. Let $K=F^{\alpha}$ endowed with the product topology $\mathcal{T}$. Then:

(a) there is a pseudocompact group topology $\mathcal{U}$ on $K$ with $w(K, \mathcal{U})=2^{|K|}$ and $\mathcal{T} \subset \mathcal{U}$ such that there is an order-isomorphism $f$ of the power set $\mathcal{P}\left(2^{|K|}\right)$, ordered by the inclusion, onto a subset of $[\mathcal{T}, \mathcal{U}]$. The compact Weil completion of $(K, \mathcal{U})$ is topologically isomorphic to $\mathrm{F}^{2^{2^{\alpha}}}$; and

(b) for each cardinal $\gamma$ such that $\alpha \leq \gamma \leq 2^{|K|}$ and for every $M \in \mathcal{P}\left(2^{|K|}\right)$ with $|M|=\gamma$, one has $w(K, f(M))=\gamma$.

From the proof of [98] (3.36) this statement follows: let $(K, \mathcal{T})$ be a profinite group with $w(K, \mathcal{T})=\alpha$. If $\operatorname{cf}(\alpha)>\omega$, there is a subnormal series $H_{1} \subset H_{2} \ldots \subset$ $H_{k}=K$ of open subgroups of $K$ such that there is a pseudocompact group topology $\mathcal{U}$ on $H_{1}$, finer than the topology induced by $\mathcal{T}$ on $H_{1}$, such that $w\left(H_{1}, \mathcal{U}\right)=2^{|G|}$. By [98] ((3.36)(b)) there is a linear totally bounded group topology $\mathcal{V}$ of weight $2^{|G|}$ on $K$ which is finer than $\mathcal{T}$ (this is constructed using the topology $\mathcal{U}$ and the subnormal series). It remains open if $\mathcal{V}$ can be chosen pseudocompact. It is natural to pose the following

Problem 8.2.11. ${ }^{*}$ Let $(K, \mathcal{T})$ be a profinite group of uncountable weight.

(a) Does $\mathcal{T}$ admit a proper pseudocompact refinement of maximal weight $2^{|K|}$ ?

(b) Are there $2^{2^{|K|}}$-many pseudocompact group topologies on $K$ which are finer than $\mathcal{T}$ ?

Acknowledgments: With many thanks, the authors acknowledge insightful, knowledgeable reports received from two referees. Collectively they have helped us enhance both the scope of the paper and its style of presentation, and with great clarity, they helped us identify some factual errors which were present in the earliest version.

Author Contributions: W. W. Comfort and Dieter Remus contributed equally to this work.

Conflicts of Interest: The authors declare no conflict of interest. 


\section{References}

1. Engelking, R. General Topology; Heldermann Verlag: Berlin, Germany, 1989.

2. Hewitt, E.; Ross, K.A. Abstract Harmonic Analysis I; Springer-Verlag: Heidelberg, Germany, 1963.

3. Hewitt, E. Rings of real-valued continuous functions I. Trans. Am. Math. Soc. 1948, 64, 45-99.

4. Comfort, W.W.; Robertson, L.C. Cardinality constraints for pseudocompact and for totally dense subgroups of compact Abelian groups. Pac. J. Math. 1985, 119, 265-285.

5. Glicksberg, I. The representation of functionals by integrals. Duke Math. J. 1952, 19, 253-261.

6. Gillman, L.; Jerison, M. Rings of Continuous Functions; D. Van Nostrand Co.: New York, NY, USA, 1960.

7. Weil, A. Sur les Espaces à Structure Uniforme et sur la Topologie Générale; Hermann \& Cie: Paris, France, 1938.

8. Comfort, W.W.; Ross, K.A. Pseudocompactness and uniform continuity in topological groups. Pac. J. Math. 1966, 16, 483-496.

9. Mycielski, J. Some properties of connected compact groups. Colloq. Math. 1958, 5, 162-166.

10. Wilcox, H.J. Dense subgroups of compact groups. Proc. Am. Math. Soc. 1971, 28, 578-580.

11. Tkachenko, M.G. Compactness type properties in topological groups. Czechoslov. Math. J. 1988, 38, 324-341.

12. Uspenskii, V.V. Topological groups and Dugundji compacta. Math. USSA Sb. 1990, 67, 555-580.

13. Hernández, S.; Sanchis, M. $G_{\delta}$-open functionally bounded subsets in topological groups. Topol. Appl. 1993, 53, 289-299.

14. Arhangel'skiı̌, A.V. On a theorem of W. W. Comfort and K. A. Ross. Comment. Math. Univ. Carol. 1999, 40, 133-151.

15. Kakutani, S.; Kodaira, K. Über das Haarsche Maß in der lokal bikompakten Gruppe. Proc. Imp. Acad. Tokyo 1944, 20, 444-450.

16. Halmos, P.R. Measure Theory; Springer-Verlag: New York, NY, USA, 1974.

17. Ross, K.A.; Stromberg, K.R. Baire sets and Baire measures. Ark. Matemat. 1967, 6, 151-160.

18. De Vries, J. Pseudocompactess and the Stone-Čech compactification for topological groups. Nieuw Arch. Wisk. 1975, 23, 35-48.

19. Hušek, M. Productivity of properties of topological groups. Topol. Appl. 1994, 44, 189-196.

20. Tkachenko, M.G. Boundedness and pseudocompactness in topological groups. Math. Notes 1987, 41, 299-231.

21. Comfort, W.W.; Trigos-Arrieta, F.J. Locally pseudocompact topological groups. Topol. Appl. 1995, 62, 263-280.

22. Dikranjan, D.; Lukács, G. On zero-dimensionality and the connected component of locally pseudocompact groups. Proc. Am. Math. Soc. 2011, 139, 2995-3008.

23. Van Douwen, E.K. The weight of a pseudocompact (homogeneous) space whose cardinality has countable cofinality. Proc. Am. Math. Soc. 1980, 80, 678-682. 
24. Comfort, W.W. Topological Groups. In Handbook of Set-theoretic Topology; Kunen, K., Vaughan, J.E., Eds.; North-Holland: Amsterdam, the Netherlands, 1984; pp. 1143-1263.

25. Comfort, W.W.; van Mill, J. Concerning connected, pseudocompact Abelian groups. Topol. Appl. 1989, 33, 21-45.

26. Hewitt, E.; Ross, K.A. Abstract Harmonic Analysis II; Springer-Verlag: Heidelberg, Germany, 1970.

27. Cater, F.S.; Erdős, P.; Galvin, F. On the density of $\lambda$-box products. Gen. Topol. Appl. 1978, 9, 307-312.

28. Comfort, W.W.; van Mill, J. Some topological groups with, and some without, proper dense subgroups. Topol. Appl. 1991, 41, 3-15.

29. Itzkowitz, G.L. Extensions of Haar measure for compact connected Abelian groups. Bull. Am. Math. Soc. 1965, 71, 152-156.

30. Wilcox, H.J. Pseudocompact groups. Pac. J. Math. 1966, 19, 365-379.

31. Comfort, W.W.; Saks, V. Countably compact groups and finest totally bounded topologies. Pac. J. Math. 1973, 49, 33-44.

32. Rajagopalan, M.; Subrahmanian, H. Dense subgroups of locally compact groups. Colloq. Math. 1976, 35, 289-292.

33. Comfort, W.W.; Ross, K.A. Topologies induced by groups of characters. Fundam. Math. 1964, 55, 283-291.

34. Comfort, W.W.; Robertson, L.C. Extremal phenomena in certain classes of totally bounded groups. Diss. Math. 1988, 272, 1-48.

35. Comfort, W.W. Tampering with pseudocompact groups. Topol. Proc. 2004, 28, 401-424.

36. Comfort, W.W.; Soundararajan, T. Pseudocompact group topologies and totally dense subgroups. Pac. J. Math. 1982, 100, 61-84.

37. Comfort, W.W.; Robertson, L.C. Proper pseudocompact extensions of compact Abelian group topologies. Proc. Am. Math. Soc. 1982, 86, 173-178.

38. Comfort, W.W. Problems on topological groups and other homogeneous spaces. In Open Problems in Topology; van Mill, J., Reed, G.M., Eds.; North-Holland Publishing Company: Amsterdam, the Netherlands, 1990; pp. 313-347.

39. Comfort, W.W. Pseudocompact groups: Progress and problems. Topol. Appl. 2008, 155, 172-179.

40. Comfort, W.W.; Remus, D. Pseudocompact refinements of compact group topologies. Math. Z. 1994, 215, 337-346.

41. Comfort, W.W.; Gladdines, H.; van Mill, J. Proper pseudocompact subgroups of pseudocompact Abelian groups. Ann. N. Y. Acad. Sci. 1994, 728, 237-247.

42. Comfort, W.W.; Galindo, J. Extremal pseudocompact topological groups. J. Pure Appl. Algebra 2005, 197, 59-81.

43. Galindo, J. Dense pseudocompact subgroups and finer pseudocompact group topologies. Sci. Math. Jpn. 2002, 55, 627-640.

44. Comfort, W.W.; van Mill, J. Extremal pseudocompact abelian groups are compact metrizable. Proc. Am. Math. Soc. 2007, 135, 4039-4044. 
45. Comfort, W.W.; van Mill, J. Extremal pseudocompact abelian groups: A unifed treatment. Comment. Math. Univ. Carol. 2013, 54, 197-227.

46. Comfort, W.W.; Galindo, J. Pseudocompact topological group refinements of maximal weight. Proc. Am. Math. Soc. 2003, 131, 1311-1320.

47. Comfort, W.W.; Remus, D. Long chains of topological group topologies-A continuation. Topol. Appl. 1997, 75, 51-79.

48. Comfort, W.W.; Remus, D. Intervals of totally bounded group topologies. Ann. N. Y. Acad. Sci. 1996, 806, 121-129.

49. Remus, D. Minimal and precompact group topologies on free groups. J. Pure Appl. Algebra 1990, 70, 147-157.

50. Dikranjan, D.N.; Shakhmatov, D.B. Compact-like totally dense subgroups of compact groups. Proc. Am. Math. Soc. 1992, 144, 1119-1129.

51. Grant, D.L. Topological Groups Which Satisfy an Open Mapping Theorem. Pac. J. Math. 1997, 68, 411-423.

52. Comfort, W.W.; Dikranjan, D. On the poset of totally dense subgroups of compact groups. Topol. Proc. 1999, 24, 103-127.

53. Comfort, W.W.; Dikranjan, D. Essential density and total density in topological groups. J. Group Theory 2002, 5, 325-350.

54. Comfort, W.W.; Dikranjan, D. The density nucleus of a topological group. Topol. Proc. 2014, 44, 325-356.

55. Comfort, W.W.; Grant, D.L. Cardinal invariants, pseudocompactness and minimality: Some recent advances in the topological theory of topological groups. Topol. Proc. 1981, 6, 227-265.

56. Giordano Bruno, A. Extremal Pseudocompact Groups. Ph.D. Thesis, Università di Udine, Udine, Italy, 2004.

57. Dikranjan, D.; Giordano Bruno, A. Pseudocompact totally dense subgroups. Proc. Am. Math. Soc. 2008, 136, 1093-1103.

58. Comfort, W.W.; van Mill, J. On the supremum of the pseudocompact group topologies. Topol. Appl. 2008, 155, 213-224.

59. Giordano Bruno, A. Extremal $\alpha$-pseudocompact abelian groups. Forum Math. 2009, 21, 639-659.

60. Dikranjan, D.; Giordano Bruno, A.; Milan, C. Weakly metrizable pseudocompact groups. Appl. Gen. Topol. 2006, 7, 1-39.

61. Giordano Bruno, A. Dense minimal subgroups of compact abelian groups. Topol. Appl. 2008, 155, 1919-1928.

62. Comfort, W.W.; van Mill, J. On the existence of free topological groups. Topol. Appl. 1988, 29, 245-269.

63. Tkachenko, M.G. Pseudocompact topological groups and their properties. Sib. Math. J. 1989, 30, 120-128.

64. Ursul, M.I. Imbeddings of locally precompact groups in locally pseudocompact ones. Izv. Akad. Nauk Mold. SSR 1989, 3, 54-56. 
65. Leiderman, A.; Morris, S.A.; Tkachenko, M.G. Density character of subgroups of topological groups. Trans. Am. Math. Soc. 2015, doi:10.1090/tran/6832.

66. Dikranjan, D.N.; Shakhmatov, D.B. Algebraic structure of pseudocompact groups. Mem. Am. Math. Soc. 1998, 633, 1-83.

67. Zel'manov, E.I. On periodic compact groups. Israel J. Math. 1992, 77, 83-95.

68. Dikranjan, D. Chains of pseudocompact group topologies. J. Pure Appl. Algebra 1998, 124, 65-100.

69. Comfort, W.W.; Raczkowski, S.U.; Trigos-Arrieta, F.J. Making group topologies with, and without, convergent sequences. Appl. Gen. Topol. 2006, 7, 109-124.

70. Itzkowitz, G.L. Cardinal numbers asociated with dense pseudocompact, countably compact, and $\omega$-bounded subgroups. Topol. Appl. 1998, 84, 21-32.

71. Hernández, S.; Hofmann, K.H.; Morris, S.A. Nonmeasurable subgroups of compact groups. J. Group Theory 2016, 19, 179-189.

72. Brian, W.R.; Mislove, M.W. Every compact group can have a non-measurable subgroup. 2015, arXiv:1503.01385.

73. Itzkowitz, G.; Shakhmatov, D. Large families of dense pseudocompact subgroups of compact groups. Fundam. Math. 1995, 147, 197-212.

74. Itzkowitz, G.L.; Shakhmatov, D. Dense countably compact subgroups of compact groups. Math. Jpn. 1997, 45, 497-501.

75. Itzkowitz, G.L.; Shakhmatov, D. Haar non-measurable partitions of compact groups. Tsukuba J. Math. 1997, 21, 251-262.

76. Varopoulos, N.T. A theorem on the continuity of homomorphisms of locally compact groups. Math. Proc. Camb. Philos. Soc. 1964, 60, 449-463.

77. Comfort, W.W.; van Mill, J. How many $\omega$-bounded subgroups? Topol. Appl. 1997, 77, 105-113.

78. Van der Waerden, B.L. Stetigkeitssätze für halbeinfache Liesche Gruppen. Math. Z. 1933, 36, 780-786.

79. Yu, Y.K. Topologically complete semisimple groups. Proc. Lond. Math. Soc. 1976, 33, 515-534.

80. Dikranjan, D.N.; Prodanov, I.R.; Stoyanov, L.N. Topological Groups (Characters, Dualities and Minimal Group Topologies); Marcel Dekker, Inc.: New York, NY, USA, 1990.

81. Hofmann, K.H.; Morris, S.A. The Structure of Compact Groups; A Primer for Students, A Handbook for Experts, 3rd ed.; Walter De Gruyter Inc.: Berlin, Germany, 2013.

82. Markov, A.A. On free topological groups. Dokl. Akad. Nauk SSSR 1941, 31, 299-301.

83. Markov, A.A. On Free Topological Groups, Topology and Topological Algebra, Translations Series 1; American Mathematical Society: Providence, RI, USA, 1962; volume 8, pp. 195-272.

84. Graev, M.I. Free Topological Groups. Izv. Akad. Nauk SSSR Ser. Mat. 1948, 12, 279-323.

85. Graev, M.L. On free products of topological groups. Izv. Akad. Nauk SSSR Ser. Mat. 1950, 14, 343-354.

86. Smith-Thomas, B.V. Free topological groups. Topol. Appl. 1974, 4, 51-72.

87. Smith-Thomas, B.V. Categories of topological groups. Quaest. Math. 1977, 2, 355-377. 
88. Kakutani, S. Free topological groups and infinite direct product topological groups. Proc. Imp. Acad. Tokyo 1944, 20, 595-598.

89. Nakayama, T. Note on free topological groups. Proc. Imp. Acad. Tokyo 1943, 19, 471-475.

90. Samuel, P. On universal mappings and free topological groups. Bull. Am. Math. Soc. 1948, 54, 591-598.

91. Morris, S.A. Free Abelian topological groups. In Proceedings of the 1983 University of Toledo Ohio Conference on Categorical Topology, Toledo, OH, USA, 1-5 August 1983.

92. Comfort, W.W.; Hofmann, K.-H.; Remus, D. Topological groups and semigroups. In Recent Progress in General Topology; Hušek, M., van Mill, J., Eds.; Elsevier Science Publishers: Amsterdam, the Netherlands, 1992; pp. 57-144.

93. Heyer, H. Dualität lokalkompakter Gruppen; Springer-Verlag: Heidelberg, Germany, 1970.

94. Poguntke, D. Zwei Klassen lokalkompakter maximal fastperiodischer Gruppen. Monatsh. Math. 1976, 81, 15-40.

95. Comfort, W.W.; Remus, D.; Szambien, H. Extending ring topologies. J. Algebra 2000, 232, 21-47.

96. Hofmann, K.H.; Morris, S.A. Weight and c. J. Pure Appl. Algebra 1990, 68, 181-194.

97. Hofmann, K.H.; Morris, S.A. A structure theorem on compact groups. Math. Proc. Camb. Philos. Soc. 2001, 130, 409-426.

98. Remus, D. Anzahlbestimmungen von gewissen präkompakten bzw. nicht-präkompakten hausdorffschen Gruppentopologien; Habilitationsschrift Universität Hannover: Hannover, Germany, 1995. 


\title{
Fixed Points of Local Actions of Lie Groups on Real and Complex 2-Manifolds
}

\author{
Morris W. Hirsch
}

Abstract: I discuss old and new results on fixed points of local actions by Lie groups $G$ on real and complex 2-manifolds, and zero sets of Lie algebras of vector fields. Results of E. Lima, J. Plante and C. Bonatti are reviewed.

Reprinted from Axioms. Cite as: Hirsch, M.W. Fixed Points of Local Actions of Lie Groups on Real and Complex 2-Manifolds. Axioms 2016, 4, 313-320.

\section{Introduction}

Classical results of Poincaré [1] (1885), Hopf [2] (1925) and Lefschetz [3] (1937) yield the archetypal fixed point theorem for Lie group actions:

Theorem 1. Every flow on a compact manifold of non-zero Euler characteristic has a fixed point.

Here the Lie group is the group $\mathbb{R}$ of real numbers.

The earliest papers I have found on fixed points for actions of other non-discrete Lie group are those of P. A. Smith [4] (1942) and H. Wang [5] (1952). Then came Armand Borel's landmark paper of 1956:

Theorem 2 (Borel [6]). If $H$ is a solvable, irreducible affine algebraic group over an algebraically closed field $\mathbb{K}$, every algebraic action of $H$ on a complete algebraic variety over $\mathbb{K}$ has a fixed point.

Over the field of complex numbers, completeness is equivalent to compactness in the classical topology, and complete nonsingular varieties are compact Kähler manifolds.

In 1973, A. Sommese [7] extended Borel's theorem to solvable holomorphic actions on compact Kähler manifolds with first Betti number 0. In contrast to the results below, these have no explicit restrictions on dimensions or Euler characteristics.

\section{Actions and Local Actions}

If $f: A \rightarrow B$ denotes a map, its domain is $\mathcal{D} f:=A$ and its range is $\mathcal{R} f:=f(A)$. Let $g, f$ denote maps. Regardless of their domains and ranges, the composition $g \circ f$ is defined as the map $x \mapsto g(f(x))$ whose domain, perhaps empty, is $f^{-1}(\mathcal{D} g)$. 
The associative law holds for these compositions: The maps $(h \circ g) \circ f$ and $h \circ(g \circ f)$ have the same domain

$$
D:=\{x \in \mathcal{D} f: f(x) \in \mathcal{D} g, \quad g(f(x)) \in \mathcal{D} f\},
$$

and

$$
x \in D \Longrightarrow(h \circ g)(f(x))=h((g \circ f)(x)) .
$$

Henceforth $M$ denotes a manifold with boundary $\partial M$, and $G$ denotes a connected Lie group with Lie algebra $\mathfrak{g}$.

A local homeomorphism $f$ on $M$ is a homeomorphism between open subsets of $M$. The set of these homeomorphisms is denoted by $\mathrm{LH}(M)$.

A local action of $G$ on $M$ is a triple $(\alpha, G, M)$, where $\alpha: G \rightarrow \operatorname{LH}(M)$ is a function having the following properties:

- The set $\Omega(\alpha):=\{(g, p) \in G \times M: p \in \mathcal{D} \alpha(g)\}$ is an open neighborhood of $\left\{e_{G}\right\} \times \mathrm{M}$.

- The evaluation map

$$
\mathrm{ev}_{\mathrm{ff}}: \Omega(\alpha) \rightarrow M, \quad(g, p) \mapsto \alpha(g) \cdot p
$$

is continuous.

- $\alpha\left(e_{G}\right)$ is the identity map of $M$.

- The maps $\alpha(f g) \circ \alpha(h)$ and $\alpha(f) \circ \alpha(g h)$ agree on the intersection of their domains.

- $\alpha\left(g^{-1}\right)=\alpha(g)^{-1}$.

Notation of $\alpha$ may be omitted.

When $\Omega(\alpha)=G \times M$ the local action is a global action. If $G$ is simply connected and $M$ is compact, every local action extends to a unique global action.

When $\alpha$ has been specified, we define the fixed-point sets

$$
\begin{aligned}
& \operatorname{Fix}(g):=\{x \in \mathcal{D} g: g(x)=x\}, \\
& \operatorname{Fix}(G):=\bigcap_{g \in G} \operatorname{Fix}(g)
\end{aligned}
$$

The local action is effective if $\operatorname{Fix}(g) \neq M$ for all $g \neq e_{G}$.

A local flow is a local action $(\Psi, \mathbb{R}, M)$. In this case we set $\Psi_{t}:=\Psi(t)$ and identify $\Psi$ with the indexed family of $\left\{\Psi_{t}\right\}_{t \in \mathbb{R}}$ of local maps in $M$. If $(\alpha, G, M)$ is a local action, to every $X \in \mathfrak{g}$ there corresponds a local flow $\left(\alpha^{*} \Psi, \mathbb{R}, M\right)$ defined in the following. Consider $X$ as a 1-parameter subgroup of $G$, i.e., a homomorphism $X: \mathbb{R} \rightarrow G$, and set $\alpha^{*} \Psi=\{\alpha(X(t))\}_{t \in \mathbb{R}}$. The local flow induced by a $C^{1}$ vector field $X$ on $M$ tangent to $\partial M$ is denoted by $\Phi^{X}:=\left\{\Phi_{t}^{X}\right\}_{t \in \mathbb{R}}$. 
A block for a local flow $\Psi$ (a $\Psi$-block) is a compact $K \subset \operatorname{Fix}(\Psi)$ having a precompact open neighborhood $U \subset M$, termed isolating, such that $\operatorname{Fix}(\Psi) \cap \bar{U}=K$. When this holds, the index $\mathrm{i}(\Psi, U)$ of $\Psi$ in $U$ is defined as the fixed point index of $\Psi_{t} \mid U: U \rightarrow M$ for sufficiently small $t>0$, as defined by Dold [8] (see also Brown [9] and Granas and Dugundji [10]). This integer depends only on $K$, and we set $i_{K}(\Psi):=i(\Psi, U)$. When $i_{K}(\Psi) \neq 0$ then $K$ is essential. If $K$ is a block for the local flow $\Phi^{X}$ of a vector field $X$, an equivalent definition of $i_{K}\left(\Phi^{X}\right)$ as the Poincaré-Hopf index of $X$ at $K$ is given in Section 4 .

\section{Fixed Points of Local Actions on Surfaces}

In the rest of this section $M$ denotes a real closed surface (compact with empty boundary) and $G$ is a connected Lie group acting continuously on $M$.

An important role is played by the group $S T_{\circ}(n, \mathbb{R})$, the solvable group of real, upper triangulable $n \times n$ matrices with positive diagonal entries. In his pioneering 1964 paper, E. Lima [11] constructed fixed-point free actions of $S T_{\circ}(2, \mathbb{R})$ on the compact 2-cell and the 2-sphere, but he also showed that every abelian Lie group action on a compact surface $M$ of nonzero Euler characteristic $\chi(M)$ has a fixed point. These results were extended in 1986 by Plante:

Theorem 3 (Plante [12]). Let M be a compact surface whose boundary may be nonempty.

(i) $S T_{\circ}(2, \mathbb{R})$ has a fixed-point free action on $M$.

(ii) If $\chi(M) \neq 0$, every action on $M$ by a connected nilpotent Lie group has a fixed point.

Many facts about existence of fixed points for continuous actions on closed surfaces can be derived from the results of $\mathrm{M}$. Belliart summarized in the following theorem. If $H \subset G L(n, \mathbb{F})$ denotes a group of matrices, $P H$ denotes the quotient of $H$ by its center.

Theorem 4 (Belliart [13]). There is a fixed-point free action of $G$ on $M$ iff one of the following conditions (a), (b), (c) holds:

(a) $\chi(M)>0$ and $G$ is solvable but not nilpotent.

(b) $\chi(M)<0$ and $G$ has $S T_{\circ}(2, \mathbb{R})$ as a quotient.

(c) $\chi(M) \geq 0$, $G$ is semisimple, and either:

(i) G has $\operatorname{PSL}(2, \mathbb{R})$ as a quotient, or

(ii) $\chi(M)>0, \partial M=\varnothing$, and $G$ has as a quotient one of the groups $\operatorname{PSL}(3, \mathbb{R}), \operatorname{PSL}(2, \mathbb{C})$ or $\operatorname{PSO}(3)$.

A Lie algebra is supersolvable if it is faithfully represented as upper triangular real matrices. A Lie group is supersolvable if its Lie algebra is. 


\section{Theorem 5.}

(i) $S T_{\circ}(3, \mathbb{R})$ has an effective analytic action on $M$.

(ii) If $G$ has an effective, fixed-point free analytic action on $M$, then $\chi(M) \geq 0$, with equality when $G$ is a supersolvable and $\partial M=\varnothing$.

Part (i) and the first conclusion in (ii) are due to Turiel [14]. The second conclusion in (ii) is due to Hirsch and Weinstein [15].

The following result gives upper and lower bounds on the number of fixed points of analytic actions of $S T_{\circ}(3, \mathbb{R})$ :

Proposition 1 (Hirsch [16], Cor. 17, Thm 22).

(i) Let $M$ have genus $g$. For every $k \in \mathbb{N}$ there is an effective analytic action $\beta$ of $S T_{\circ}(3, \mathbb{R})$ on $M$ such that:

$$
\# \operatorname{Fix}(\beta)= \begin{cases}2(g+k+1) & \text { if } M \text { is orientable, } \\ g+k & \text { if } M \text { is nonorientable and } g \geq 1\end{cases}
$$

(ii) If $G$ is not supersolvable and has an effective analytic action on $M$,

$$
0 \leq \# \operatorname{Fix}(G) \leq \chi(M) \leq 2
$$

Question. Can the right hand side of Equation (1) can be lowered?

\section{Indices of Vector Fields}

Let $\mathcal{V}(M)$ denote the vector space of vector fields (continuous sections of the tangent bundle) on a smooth manifold $M$, endowed with the compact open topology.

The zero set of $X \in \mathcal{V}(M)$ is

$$
\mathrm{Z}(X):=\left\{p \in M: X_{p}=0\right\}
$$

A block for $X$ (an $X$-block) is a compact, relatively open set $K \subset \mathrm{Z}(X)$. Every sufficiently small open neighborhood $U \subset M$ of $K$ is isolating for $X$, meaning its closure $\bar{U}$ is compact and $Z(X) \cap \bar{U}=K$. This implies that $U$ is isolating for every vector field $Y$ sufficiently close to $X$.

Let $K$ be an $X$-block. When $K$ is finite, the Poincaré-Hopf index of $X$ at $K$, and in $U$, is the integer $i_{K}^{P H}(X)=\mathrm{i}^{P H}(X, U)$ defined as follows. For each $p \in K$ choose an open set $W \subset U$ meeting $K$ only at $p$, such that $W$ is the domain of a $C^{1}$ chart

$$
\phi: W \approx W^{\prime} \subset \mathbb{R}^{n}, \quad \phi(p)=p^{\prime} .
$$


The transform of $X$ by $\phi$ is

$$
X^{\prime}:=T \phi \circ X \circ \phi^{-1} \in \mathcal{V}\left(W^{\prime}\right)
$$

There is a unique map of pairs

$$
\left.F_{p}:\left(W^{\prime}, 0\right) \rightarrow \mathbb{R}^{n}, 0\right)
$$

that expresses $X^{\prime}$ by the formula

$$
X_{x}^{\prime}=\left(x, F_{p}(x)\right) \in\{x\} \times \mathbb{R}^{n}, \quad\left(x \in W^{\prime}\right) .
$$

Noting that $F^{-1}(0)=p$, we define $\mathrm{i}_{p}^{P H}(X) \in \mathbb{Z}$ as the degree of the map defined for any sufficiently small $\epsilon>0$ as

$$
\mathbf{S}^{n-1} \rightarrow \mathbf{S}^{n-1}, \quad u \mapsto \frac{F_{p}(\epsilon u)}{\left\|F_{p}(\epsilon u)\right\|}
$$

where $\|\cdot\|$ is the norm defined by any Riemannian metric on $M$. This degree is independent of $\epsilon$ and the chart $\phi$, by standard properties of the degree function. Therefore the integer

$$
\mathrm{i}_{K}^{P H}(X)=i^{P H}(X, U):= \begin{cases}\sum_{p \in K} i_{p}^{P H}(X) & \text { if } K \neq \varnothing, \\ 0 & \text { if } K=.\end{cases}
$$

is well defined and depends only on $X$ and $K$.

The index of an arbitrary $X$-block $K$ is the integer $i_{K}(X):=\mathrm{i}(X, U)$ defined as the Poincaré-Hopf index of any sufficiently close approximation to $X$ having only finitely many zeros in $U$ [17].

This number is independent of $U$ and is stable under perturbations of $X$. The $X$-block $K$ is essential when $i_{K}(X) \neq 0$. This implies $Z(X) \cap K \neq \varnothing$ because every isolating neighborhood of $K$ meets $Z(X)$.

Theorem 6 (Poincaré-Hopf). If $M$ is compact, $\mathrm{i}(X, M)=\chi(M)$ for all continuous vector fields $X$ on $M$.

For calculations of the index in more general settings see Morse [18], Pugh [19], Gottlieb [20], Jubin [21].

Theorem 7 (Bonatti [22]). Assume $M$ is a real manifold of dimension $\leq 4$ with empty boundary, and $X, Y$ are analytic vector fields on $M$ such that $[X, Y]=0$. Then $Z(Y)$ meets every essential X-block [23]. 
This implies certain local actions of 2-dimensional abelian Lie groups have fixed points. The results below are analogs for local actions of nonabelian Lie groups.

Theorem 8 (Hirsch [24]). Let $M$ be a real surface, perhaps non-compact or having non-empty boundary. Let $G$ be a connected nilpotent Lie group and $(\alpha, G, M)$ an effective local action. Assume given a continuous local action of $G$ on $M$, and let $K$ be an essential block for the local flow induced by a 1-parameter subgroup. Then $\operatorname{Fix}(G) \cap K \neq \varnothing$.

This implies Plante's result, Theorem 3(ii).

Corollary 1. Let $G, M$ and $X$ be as in Theorem 8 .

(i) If $\Gamma \subset M$ is a compact attractor for $\Phi^{X}$ and $\chi(\Gamma) \neq 0$, then $\operatorname{Fix}(G) \cap \Gamma \neq \varnothing$.

(ii) If $\Phi^{X}$ has $n$ essential blocks, then $\operatorname{Fix}(G)$ has $n$ components.

The counter-example in Theorem 3(i) show that fixed point results for broader classes of Lie groups, including supersolvable groups, need stronger hypotheses.

Henceforth $M$ denotes either a real or complex 2-manifold, the corresponding ground field being $\mathbb{F}=\mathbb{R}$ or $\mathbb{C}$. Let $\mathcal{V}^{\omega}(M)$ denote the Lie algebra of vector fields on $M$ that are analytic over $\mathbb{F}$. If $Y \in \mathcal{V}^{\omega}(M), T \Phi^{Y}$ denotes the induced local flow on the tangent vector bundle of $M$.

Assume $X, Y \in \mathcal{V}^{\omega}(M)$. We say that $Y$ tracks $X$ if there exists a continuous map

$$
f: M \rightarrow \mathbb{F}, \quad f^{-1}(0)=Z(X), \quad[Y, X]=f X
$$

Equivalently: if $p \in M$ and $t \in \mathbb{R}$ there exists $g(t, p) \in \mathbb{F}$ such that:

$$
\Phi_{t}^{Y}(p)=q(t) \Longrightarrow T \Phi_{t}^{Y}\left(X_{p}\right)=g(t, p) X_{q(t)}
$$

For real $M$ this means $\Phi_{t}^{Y}$ sends orbits of $X \mid \mathcal{D} \Phi_{t}^{Y}$ to orbits of $X \mid \mathcal{R} \Phi_{t}^{Y}$.

Let $\mathcal{G} \subset \mathcal{V}^{\omega}(M)$ denote a Lie algebra of vector fields. We say that $\mathcal{G}$ tracks $X$ provided each $Y \in \mathcal{G}$ tracks $X$.

Example 1. If $X$ spans an ideal in $\mathcal{G}$ then $\mathcal{G}$ tracks $X$, and the converse holds if $\mathcal{G}$ is finite dimensional.

Example 2. The set $\left\{Y \in \mathcal{V}^{\omega}(M): Y\right.$ tracks $\left.X\right\}$ is a Lie algebra that tracks $X$.

The following result will be proved in a forthcoming paper [25]; a preliminary version is in [26].

Theorem 9. Assume $X \in \mathcal{V}^{\omega}(M), K$ is an essential $X$-bloc, and $\mathcal{G} \subset \mathcal{V}^{\omega}(M)$ tracks $X$. Let one of the following conditions hold: 
(a) $M$ is complex,

(b) $M$ is real and $\mathcal{G}$ is supersolvable.

Then $\mathrm{Z}(\mathcal{G}) \cap K \neq \varnothing$.

Example 3. Here is a simple example in which the hypotheses hold. For $M$ take complex projective 3-space. Let $G$ be the solvable complex Lie group of unimodular $4 \times 4$ upper triangular complex matrices. The natural action of $G$ on $\mathbb{C} 4$ induces an effective holomorphic action of $G$ on $M$, mapping the Lie algebra of $G$ isomorphically onto a Lie algebra $\mathcal{G} \subset \mathcal{V}^{\omega}(M)$. Let $X \in \mathcal{G}$ have the block $\left[\begin{array}{ll}0 & 1 \\ 0 & 0\end{array}\right]$ in its upper right hand corner and all other elements equal to zero. $X$ spans an ideal, the triple commutator subalgebra $\mathcal{G}^{\prime \prime \prime}$. The $X$-block $K:=Z(X)$, a copy of $\mathbb{C} \mathbf{P}^{1}$, is essential because $\chi(M)=3$; and $Z(\mathcal{G})$ is a singleton in $Z(X)$.

Conflicts of Interest: The author declares no conflict of interest.

\section{References}

1. Poincaré, H. Sur les courbes définies par une équation différentielle. J. Math. Pures Appl. 1885, 1, 167-244.

2. Hopf, H. Vektorfelder in Mannifgfaltigkeiten. Math. Ann. 1925, 95, 340-367.

3. Lefschetz, S. On the fixed point formula. Ann. Math. 1937, 38, 819-822.

4. Smith, P.A. Stationary points of transformation groups. Proc. Natl. Acad. Sci. USA 1942, 28, 293-297.

5. Wang, H.-C. A remark on transformation groups leaving fixed an end point. Proc. Am. Math. Soc. 1952, 3, 548-549.

6. Borel, A. Groupes linéaires algébriques. Ann. Math. 1956, 64, 20-80.

7. Sommese, A. Borel's fixed point theorem for Kaehler manifolds and an application. Proc. Am. Math. Soc. 1973, 41, 51-54.

8. Dold, A. Lectures on Algebraic Topology. In Die Grundlehren der Matematischen Wissenschaften Bd. 52, 2nd ed.; Springer-Verlag: New York, NY, USA, 1972.

9. Brown, R. The Lefschetz fixed point theorem. Scott, Foresman \& Co.: Glenview, IL, USA, 1971.

10. Granas, A.; Dugundji, J. Fixed Point Theory; Springer-Verlag: New York, NY, USA, 2003.

11. Lima, E. Common singularities of commuting vector fields on 2-manifolds. Comment. Math. Helv. 1964, 39, 97-110.

12. Plante, J. Fixed points of Lie group actions on surfaces. Ergod. Theory Dyn. Syst. 1986, 6, 149-161.

13. Belliart, M. Actions sans points fixes sur les surfaces compactes. Math. Z. 1997, 225, 453-465.

14. Turiel, F.-J. Analytic actions on compact surfaces and fixed points. Manuscr. Math. 2003, 110, 195-201.

15. Hirsch, M.; Weinstein, A. Fixed points of analytic actions of supersoluble Lie groups on compact surfaces. Ergod. Theory Dyn. Syst. 2001, 21, 1783-1787 
16. Hirsch, M. Actions of Lie groups and Lie algebras on manifolds. In A Celebration of the Mathematical Legacy of Raoul Bott; Amer. Math. Soc.: Providence, RI, USA, 2010.

17. Equivalently: $\mathrm{i}(X, U)$ is the intersection number of $X \mid U$ with the zero section of the tangent bundle (Bonatti [22]).

18. Morse, M. Singular Points of Vector Fields Under General Boundary Conditions. Am. J. Math. 1929, 52, 165-178.

19. Pugh, C. A generalized Poincaré index formula. Topology 1968, 7, 217-226.

20. Gottlieb, D. A de Moivre like formula for fixed point theory. In Fixed Point Theory and its Applications (Berkeley, CA, 1986); Amer. Math. Soc.: Providence, RI, USA, 1988.

21. Jubin, B. A generalized Poincaré-Hopf index theorem. arxiv:0903.0697, 2009.

22. Bonatti, C. Champs de vecteurs analytiques commutants, en dimension 3 ou 4: Existence de zéros communs. Bol. Soc. Brasil. Mat. 1992, 22, 215-247.

23. This paper was inspired by a remarkable result of $C$. Bonatti, which does not require compactness of $M$. "The demonstration of this result involves a beautiful and quite difficult local study of the set of zeros of $X$, as an analytic $Y$-invariant set." -Molino, $\tilde{P}$. Review of Bonatti [22], Math Reviews 93h:57044; Amer. Math. Soc.: Providence, RI, USA, 1993.

24. Hirsch, M. Fixed points of local actions of nilpotent Lie groups on surfaces. arXiv:org/1405.2331, 2013.

25. Hirsch, M. Zero sets of Lie algebras of analytic vector fields on real and complex 2-manifolds. 2015, submitted.

26. Hirsch, M. Zero sets of Lie algebras of analytic vector fields on real and complex 2-manifolds. arXiv.1310.0081v2, 2015. 


\section{Free Boolean Topological Groups}

\section{Ol'ga Sipacheva}

Abstract: Known and new results on free Boolean topological groups are collected. An account of the properties that these groups share with free or free Abelian topological groups and properties specific to free Boolean groups is given. Special emphasis is placed on the application of set-theoretic methods to the study of Boolean topological groups.

Reprinted from Axioms. Cite as: Sipacheva, O. Free Boolean Topological Groups. Axioms 2016, 4, 492-517.

\section{Introduction}

In the very early 1940s, Markov [1,2] introduced the free topological group $F(X)$ and the free Abelian topological group $A(X)$ on an arbitrary completely regular Hausdorff topological space $X$ as a topological-algebraic counterpart of the abstract free and free Abelian groups on a set, respectively; he also proved the existence and uniqueness of these groups. During the next decade, Graev [3,4], Nakayama [5] and Kakutani [6] simplified the proofs of the main statements of Markov's theory of free topological groups, generalized Markov's construction and proved a number of important theorems on free topological groups. In particular, Graev generalized the notions of the free and the free Abelian topological group on a space $X$ by identifying the identity element of the free group with an (arbitrary) point of $X$ (the free topological group on $X$ in the sense of Markov coincides with Graev's group on $X$ plus an isolated point), described the topology of free topological groups on compact spaces and extended any continuous pseudometric on $X$ to a continuous invariant pseudometric on $F(X)$ (and on $A(X)$ ) which is maximal among all such extensions [3].

This study stimulated Mal'tsev, who believed that the most appropriate place of the theory of abstract free groups was in the framework of the general theory of algebraic systems, to introduce general free topological algebraic systems. In 1957, he published the large paper [7], where the basics of the theory of free topological universal algebras were presented.

Yet another decade later, Morris initiated the study of free topological groups in the most general aspect. Namely, he introduced the notion of a variety of topological groups and a full variety of topological groups and studied the free objects of these varieties [8-10] (see also [11]). (A definition of a variety of topological groups (determined by a so-called varietal free topological group) was also proposed in 1951 by Higman [12]; however, it is Morris' definition that has proven viable and 
developed into a rich theory.) Varieties of topological groups and their free objects were also considered by Porst [13], Comfort and van Mill [14], Kopperman, Mislove, Morris, Nickolas, Pestov and Svetlichny [15], and other authors. Special mention should be made of Dikranjan and Tkachenko's detailed study of varieties of Abelian topological groups with properties related to compactness [16].

The varieties of topological groups in which free objects have been studied best are, naturally, the varieties of general and Abelian topological groups; free and free Abelian precompact groups have also been considered (see, e.g., [17]). However, there is yet another natural variety: Boolean topological groups. Free objects in this variety and its subvarieties have been investigated much less extensively, although they arise fairly often in various studies (especially in the set-theoretic context). The author is aware of only three published papers considering free Boolean topological groups from a general point of view: [18], where free Boolean topological groups on compact spaces were studied fairly thoroughly; [19], where the topology of the free Boolean topological group on a compact metric space was explicitly described; and [20], where the free Boolean topological groups on compact initial segments of ordinals were classified (see also [21]). The purpose of this paper is to draw attention to these very interesting groups and to give a general impression of them. We collect some (known and new) results on free Boolean topological groups, which describe both properties that these groups share with free or free Abelian topological groups and properties specific to free Boolean groups.

\section{Preliminaries and a General Description of Free Boolean Topological Groups}

All topological spaces and groups considered in this paper are assumed to be completely regular and Hausdorff.

The notation $\omega$ is used for the set of all nonnegative integers and $\mathbb{N}$ for the set of all positive integers. By $\mathbb{Z}_{2}$, we denote the group of order two. The cardinality of a set $A$ is denoted by $|A|$ and the closure of a set $A$ in an ambient topological space by $\bar{A}$. We denote the disjoint union of spaces $X$ and $Y$ by $X \oplus Y$.

By a zero-dimensional space, we mean a space $X$ with ind $X=0$ and by a strongly zero-dimensional space a space $X$ with $\operatorname{dim} X=0$.

A Boolean group is a group in which all elements are of order two. Clearly, all Boolean groups are Abelian. Algebraically, all Boolean groups are free, because any Boolean group is a linear space over the field $\mathbb{F}_{2}=\{0,1\}$ and must have a basis (a maximal linearly independent set) by Zorn's lemma. This basis freely generates the given Boolean group. Moreover, any Boolean group (linear space) with basis $X$ is isomorphic to the direct sum $\bigoplus^{|X|} \mathbb{Z}_{2}$ of $|X|$ copies of $\mathbb{Z}_{2}$, i.e., the set of finitely supported maps $g: X \rightarrow \mathbb{Z}_{2}$ with pointwise addition (in the field $\mathbb{F}_{2}$ ). Of course, such an isomorphic representation depends on the choice of the basis. 
A variety of topological groups is a class of topological groups closed with respect to taking topological subgroups, topological quotient groups and Cartesian products of groups with the product topology. Thus, the abstract groups $\widetilde{G}$ underlying the topological groups $G$ in a variety $V$ of topological groups (that is, all groups $G \in V$ without topology) form a usual variety $\widetilde{V}$ of groups. A variety $V$ of topological groups is full if any topological group $G$ for which $\widetilde{G} \in \widetilde{V}$ belongs to $V$. The notions of a variety and a full variety of topological groups were introduced by Morris in [8,9], who also proved the existence of the free group of any full variety on any completely regular Hausdorff space $X$.

Free objects of varieties of topological groups are characterized by the corresponding universality properties (we give a somewhat specific meaning to the word "universality," but we use this word only in this meaning here). Thus, the free topological group $F(X)$ on a space $X$ admits the following description: $X$ is topologically embedded in $F(X)$ and, for any continuous map $f$ of $X$ to a topological group $G$, there exists a continuous homomorphism $\hat{f}: F(X) \rightarrow G$ for which $f=\hat{f} \uparrow X$. As an abstract group, $F(X)$ is the free group on the set $X$. The topology of $F(X)$ can be defined as the strongest group topology inducing the initial topology on $X$. On the other hand, the free topological group $F(X)$ is the abstract free group generated by the set $X$ (which means that any map of the set $X$ to any abstract group can be extended to a homomorphism of $F(X)$ ) endowed with the weakest topology with respect to which all homomorphic extensions of continuous maps from $X$ to topological groups are continuous. The free Abelian topological group $A(X)$ on $X$, the free Boolean topological group $B(X)$ on $X$ and free (free Abelian, free Boolean) precompact groups are defined similarly; instead of continuous maps to any topological groups, continuous maps to topological Abelian groups, topological Boolean groups and precompact (Abelian precompact, Boolean precompact) groups should be considered.

There is yet another family of interesting varieties of topological groups. Following Malykhin (see also [17]), we say that a topological group is linear if it has a base of neighborhoods of the identity element which consists of open subgroups. The classes of all linear groups, all Abelian linear groups and all Boolean linear groups are varieties of topological groups. These varieties are not full, but for any zero-dimensional space $X$, there exist free groups of all of these three varieties on $X$. Indeed, Morris proved that a free group of a variety of topological groups on a given space exists if this space can be embedded as a subspace in a group from this variety ([8], Theorem 2.6). Thus, it suffices to embed any zero-dimensional $X$ in a Boolean linear topological group (which belongs to all of the three varieties under consideration). We do this below, but first we introduce more notation.

Whenever $X$ algebraically generates a group $G$, we can set the length of the identity element to zero, define the length of any non-identity $g \in G$ with respect 
to $X$ as the least (positive) integer $n$ such that $g=x_{1}^{\varepsilon_{1}} x_{2}^{\varepsilon_{2}} \ldots x_{n}^{\varepsilon_{n}}$ for some $x_{i} \in X$ and $\varepsilon_{i}= \pm 1, i=1,2, \ldots, n$, and denote the set of elements of length at most $k$ by $G_{k}$ for $k \in \omega$; then, $G=\bigcup G_{k}$. Thus, we use $F_{k}(X), A_{k}(X)$ and $B_{k}(X)$ to denote the sets of words of length at most $k$ in $F(X), A(X)$ and $B(X)$, respectively.

Now, we can describe the promised embedding.

Lemma 1. (i) For any space $X$ with ind $X=0$, there exists a Hausdorff linear topological group $F^{\prime}(X)$ such that $F^{\prime}(X)$ is an algebraically free group on $X, X$ is a closed subspace of $F^{\prime}(X)$, and all sets $F_{n}(X)$ of words of length at most $n$ are closed in $F^{\prime}(X)$.

(ii) For any space $X$ with ind $X=0$, there exists a Hausdorff Abelian linear topological group $A^{\prime}(X)$ such that $A^{\prime}(X)$ is an algebraically free Abelian group on $X, X$ is a closed subspace of $A^{\prime}(X)$, and all sets $A_{n}(X)$ of words of length at most $n$ are closed in $A^{\prime}(X)$.

(iii) For any space $X$ with ind $X=0$, there exists a Hausdorff Boolean linear topological group $B^{\prime}(X)$ such that $B^{\prime}(X)$ is an algebraically free Boolean group on $X, X$ is a closed subspace of $B^{\prime}(X)$, and all sets $B_{n}(X)$ of words of length at most $n$ are closed in $B^{\prime}(X)$.

Proof. Assertion (i) was proven in [22], Theorem 10.5. Let us prove (ii). Given a disjoint open cover $\gamma$ of $X$, we set:

$$
H(\gamma)=\left\{\sum_{i=1}^{n}\left(x_{i}-y_{i}\right): n \in \mathbb{N} \text { and for each } i \leq n, \text { there exists an } U_{i} \in \gamma \text { for which } x_{i}, y_{i} \in U_{i}\right\}
$$

this is a subgroup of the free Abelian group on $X$. We can assume that all words in $H(\gamma)$ are reduced (if $x_{i}$ is canceled with $y_{j}$, then $U_{i}=U_{j}$, because $U_{i} \cap U_{j} \ni x_{i}=y_{j}$ and $\gamma$ is disjoint, and we can replace $x_{i}-y_{i}+x_{j}-y_{j}$ by $x_{j}-y_{i}$ ). All such subgroups generate a group topology on the free Abelian group on $X$; we denote the free Abelian group with this topology by $A^{\prime}(X)$ (we might as well take only finite covers).

The space $X$ is indeed embedded in $A^{\prime}(X)$ : given any clopen neighborhood $U$ of any point $x \in X$, we have $x+H(\{U, X \backslash U\}) \cap X=U$.

Let us show that $A_{n}(X)$ is closed in $A^{\prime}(X)$ for any $n \in \omega$. Take any reduced word $g=\varepsilon_{1} x_{1}+\varepsilon_{2} x_{2}+\cdots+\varepsilon_{k} x_{k}$ with $k>n$, where $\varepsilon_{i}= \pm 1$ and $x_{i} \in X$ for $i \leq k$. Let $U_{i}$ be clopen neighborhoods of $x_{i}$ such that $U_{i}$ and $U_{j}$ are disjoint if $x_{j} \neq x_{i}$ and coincide if $x_{j}=x_{j}$. We set:

$$
\gamma=\left\{U_{1}, \ldots, U_{k}, X \backslash \bigcup_{i \leq k} U_{i}\right\}
$$

Take any reduced word $h=\sum_{i=1}^{m}\left(y_{i}-z_{i}\right)$ in $H(\gamma)$ and consider $g+h$. If, for some $i \leq m$, both $y_{i}$ and $-z_{i}$ are canceled in $g+h$ with some $x_{j}$ and $x_{l}$, then, first, $x_{j}=x_{l}$ 
(because any different letters in $g$ are separated by the cover $\gamma$, while $y_{i}$ and $z_{i}$ must belong to the same element of this cover), and secondly, $\varepsilon_{j}=-\varepsilon_{l}$ (because $y_{i}$ and $z_{i}$ occur in $h$ with opposite signs). Hence, $\varepsilon_{j} x_{j}=-\varepsilon_{l} x_{l}$, which contradicts $g$ being reduced. Thus, among any two letters $y_{i}$ and $-z_{i}$ in $h$, only one can be canceled in $g+h$, so that $g+h$ cannot be shorter than $g$. In other words, $g+H(\gamma) \cap A_{n}^{\prime}(X)=\varnothing$.

The proof that $X$ is closed in $A^{\prime}(X)$ is similar: given any $g \notin X$, we construct precisely the same $\gamma$ as above (if $g \notin-X$ ) or set $\gamma=\{X\}$ (if $g \in-X$ ) and show that $g+H(\gamma)$ must contain at least one negative letter.

The Hausdorffness of $A^{\prime}(X)$ is equivalent to the closedness of $A_{0}(X)$.

The proof of (iii) is similar.

This lemma and Morris' theorem cited above ([8], Theorem 2.6) immediately imply the following theorem.

Theorem 2. For any space $X$ with ind $X=0$, the free, free Abelian and free Boolean linear topological groups $F^{\operatorname{lin}}(X), A^{\operatorname{lin}}(X)$ and $B^{\operatorname{lin}}(X)$ are defined. They are Hausdorff and contain $X$ as a closed subspace, and all sets $F_{n}(X), A_{n}(X)$ and $B_{n}(X)$ are closed in the respective groups.

By definition, the free linear groups of a zero-dimensional space $X$ have the strongest linear group topologies inducing the topology of $X$, that is, any continuous map from $X$ to a linear topological group (Abelian linear topological group, Boolean linear topological group) extends to a continuous homomorphism from $F^{\operatorname{lin}}(X)$ $\left(A^{\operatorname{lin}}(X), B^{\operatorname{lin}}(X)\right)$ to this group.

Let $X$ be a space, and let $X_{n}, n \in \omega$, be its subspaces such that $X=\cup X_{n}$. Suppose that any $Y \subset X$ is open in $X$ if and only if each $Y \cap X_{n}$ is open in $X_{n}$ (replacing "open" by "closed," we obtain an equivalent condition). Then, $X$ is said to have the inductive limit topology (with respect to the decomposition $X=\cup X_{n}$ ). When talking about inductive limit topologies on $F(X), A(X)$ and $B(X)$, we always mean the decompositions $F(X)=\bigcup F_{k}(X), A(X)=\bigcup A_{k}(X)$ and $B(X)=\bigcup B_{k}(X)$ and assume the sets $F_{k}(X), A_{k}(X)$ and $B_{k}(X)$ to be endowed with the topology induced by the respective free topological groups.

For any space $X$, the free Abelian topological group $A(X)$ is the quotient topological group of $F(X)$ by the commutator subgroup, and the free Boolean topological group $B(X)$ is the quotient of $A(X)$ by the subgroup of squares $A(2 X)$ (which is generated by all words of the form $2 x, x \in X$ ) (the universality of free objects in varieties of topological groups implies that the corresponding homomorphisms are continuous and open). Thus, $B(X)$ is the image of $A(X)$ (and of $F(X)$ ) under a continuous open homomorphism.

The topology of free groups can be described explicitly. The first descriptions were given for free topological groups on compact spaces and free Abelian topological 
groups by Graev [3,4]; Tkachenko [23,24] and Pestov [25] gave explicit descriptions of the topology of general free topological groups. There are also descriptions due to the author (see, e.g., [26,27]). Mal'tsev proposed a universal approach to describing the topology of free topological algebras, which is not quite constructive, but looks very promising [7]. All descriptions of the topology of free and free Abelian topological groups of which the author is aware are given in [22]. The descriptions of the free topological group topology are very complex (except in a few special cases); the topologies of free Abelian and Boolean topological groups look much simpler. Thanks to the fact that $B(X)=A(X) / A(2 X)$, the descriptions of the free Abelian topological group topology given in [22] immediately imply the following descriptions of the free topology of $B(X)$.

I For each $n \in \mathbb{N}$, we fix an arbitrary entourage $W_{n} \in \mathcal{U}$ of the diagonal of $X \times X$ in the universal uniformity of $X$ and set:

$$
\begin{gathered}
\widetilde{W}=\left\{W_{n}\right\}_{n \in \mathbb{N}}, \\
U\left(W_{n}\right)=\left\{x+y:(x, y) \in W_{n}\right\}, \\
U(\widetilde{W})=\bigcup_{n \in \mathbb{N}}\left(U\left(W_{1}\right)+U\left(W_{2}\right)+\cdots+U\left(W_{n}\right)\right) .
\end{gathered}
$$

The sets $U(\widetilde{W})$, where $\widetilde{W}$ ranges over all sequences of uniform entourages of the diagonal, form a neighborhood base at zero for the topology of the free Boolean topological group $B(X)$.

II For each $n \in \mathbb{N}$, we fix an arbitrary normal (or merely open) cover $\gamma_{n}$ of the space $X$ and set:

$$
\begin{gathered}
\Gamma=\left\{\gamma_{n}\right\}_{n \in \mathbb{N}} \\
U\left(\gamma_{n}\right)=\left\{x+y:(x, y) \in U \in \gamma_{n}\right\} \\
U(\Gamma)=\bigcup_{n \in \mathbb{N}}\left(U\left(\gamma_{1}\right)+U\left(\gamma_{2}\right)+\cdots+U\left(\gamma_{n}\right)\right) .
\end{gathered}
$$

The sets $U(\Gamma)$, where $\Gamma$ ranges over all sequences of normal (or arbitrary open) covers, form a neighborhood base at zero for the topology of $B(X)$.

III For an arbitrary continuous pseudometric $d$ on $X$, we set:

$$
U(d)=\left\{x_{1}+y_{1}+x_{2}+y_{2}+\cdots+x_{n}+y_{n}: n \in \mathbb{N}, x_{i}, y_{i} \in X, \sum_{i=1}^{n} d\left(x_{i}, y_{i}\right)<1\right\} .
$$

The sets $U(d)$, where $d$ ranges over all continuous pseudometrics on $X$, form a neighborhood base at zero for the topology of $B(X)$.

It follows directly from the second description that the base of neighborhoods of zero in $B^{\operatorname{lin}}(X)$ (for zero-dimensional $X$ ) is formed by the subgroups: 


$$
\langle U(\gamma)\rangle=\left\{\sum_{i=1}^{n}\left(x_{i}+y_{i}\right): n \in \mathbb{N},\left(x_{i}, y_{i}\right) \in U_{i} \in \gamma \text { for } i \leq n\right\}
$$

generated by the sets $U(\gamma)$ with $\gamma$ ranging over all normal covers of $X$. By definition, any normal cover of a strongly zero-dimensional space has a disjoint open refinement. Therefore, for $X$ with $\operatorname{dim} X=0$, the covers $\gamma$ can be assumed to be disjoint, and for disjoint $\gamma$, we have:

$$
\langle U(\gamma)\rangle=\left\{\sum_{i=1}^{n}\left(x_{i}+y_{i}\right): n \in \mathbb{N},\left(x_{i}, y_{i}\right) \in U_{i} \in \gamma \text { for } i \leq n, \text { the word } \sum_{i=1}^{n}\left(x_{i}+y_{i}\right) \text { is reduced }\right\}
$$

(see the proof of Lemma 1). A similar description is valid for the Abelian groups $A^{\operatorname{lin}}(X)$ (the pluses must be replaced by minuses). This leads to the following statement.

Proposition 3. For any strongly zero-dimensional space $X$ and any $n \in \omega$, the topology induced on $A_{n}(X)$ (on $\left.B_{n}(X)\right)$ by $A^{\text {lin }}(X)\left(\right.$ by $B^{\operatorname{lin}}(X)$ ) coincides with that induced by $A(X)$ (by $B(X)$ ).

Proof. We can assume without loss of generality that $n$ is even. Given any neighborhood $U$ of zero in $A(X)$ (in $B(X)$ ), it suffices to take a sequence $\Gamma=\left\{\gamma_{k}\right\}_{k \in \mathbb{N}}$ of disjoint covers such that $\frac{n}{2} \cdot U(\Gamma) \subset U$ and note that $\left\langle U\left(\gamma_{1}\right)\right\rangle \cap A_{n}(X) \subset$ $\frac{n}{2} \cdot U\left(\gamma_{1}\right) \subset U$.

Graev's procedure for extending any continuous pseudometric $d$ on $X$ to a maximal invariant pseudometric $\hat{d}$ on $F(X)$ is easy to adapt to the Boolean case. Following Graev, we first consider free topological groups in the sense of Graev, in which the identity element is identified with a point of the generating space and the universality property is slightly different: only continuous maps of the generating space to topological groups $G$ that take the distinguished point to the identity elements of $G$ must extend to continuous homomorphisms [3]. Graev showed that the free topological and Abelian topological groups $F_{G}(X)$ and $A_{G}(X)$ in the sense of Graev are unique (up to topological isomorphism) and do not depend on the choice of the distinguished point; moreover, the free topological group in the sense of Markov is nothing but the Graev free topological group on the same space to which an isolated point is added (and identified with the identity element).

The extension of a continuous pseudometric $d$ on $X$ to a maximal invariant continuous pseudometric $\hat{d}$ on the Graev free Boolean topological group $B_{G}(X)$ is defined by setting:

$$
\hat{d}(g, h)=\inf \left\{\sum_{i=1}^{n} d\left(x_{i}, y_{i}\right): n \in \mathbb{N}, x_{i}, y_{i} \in X, g=\sum_{i=1}^{n} x_{i}, h=\sum_{i=1}^{n} y_{i}\right\}
$$


for any $g, h \in B_{G}(X)$. The infimum is taken over all representations of $g$ and $h$ as (reducible) words of equal lengths. The corresponding Graev seminorm $\|\cdot\|_{d}$ (defined by $\|g\|_{d}=\hat{d}(g, 0)$ for $g \in B_{G}(X)$, where 0 is the zero element of $B_{G}(X)$ ) is given by:

$$
\|g\|_{d}=\inf \left\{\sum_{i=1}^{n} d\left(x_{i}, y_{i}\right): g=\sum_{i=1}^{n}\left(x_{i}+y_{i}\right), x_{i}, y_{i} \in X\right\} .
$$

The infimum is attained at a word representing $g$ which may contain one zero (if the length of $g$ is odd) and is otherwise reduced. Indeed, if the sum representing $g$ contains terms of the form $x+z$ and $z+y$, then these terms can be replaced by one term $x+y$; the sum $\sum_{i=1}^{n} d\left(x_{i}, y_{i}\right)$ does not increase under such a change thanks to the triangle inequality.

For the usual (Markov's) free Boolean topological group $B(X)$, which is the same as $B_{G}(X \oplus\{0\})$ (where 0 is an isolated point identified with zero), the Graev metric depends on the distances from the points of $X$ to the isolated point (they can be set to 1 for all $x \in X)$. The corresponding seminorm $\|\cdot\|_{d}$ on the subgroup $B_{\text {even }}(X)$ of $B(X)$ consisting of words of even length does not change. The subgroup $B_{\text {even }}(X)$ is open and closed in $B(X)$, because this is the kernel of the continuous homomorphism $\hat{f}: B(X) \rightarrow\{0,1\}$ extending the constant continuous map $f: X \rightarrow\{0,1\}$ taking all $x \in X$ to 1 . Thus, in fact, it does not matter how to extend $\|\cdot\|_{d}$ to $B(X) \backslash B_{\text {even }}(X)$; for convenience, we set:

$$
\|g\|_{d}=\left\{\begin{array}{cc}
\min \left\{\sum_{i=1}^{n} d\left(x_{i}, y_{i}\right): g=\sum_{i=1}^{n}\left(x_{i}+y_{i}\right), x_{i}, y_{i} \in X,\right. & \text { if } g \in B_{\text {even }}(X), \\
\text { the word } \left.\sum_{i=1}^{n}\left(x_{i}+y_{i}\right) \text { is reduced }\right\} & \\
1 & \text { if } g \in B(X) \backslash B_{\text {even }}(X) .
\end{array}\right.
$$

All open balls (as well as all open balls of any fixed radius not exceeding one) in all seminorms $\|\cdot\|_{d}$ for $d$ ranging over all continuous pseudometrics on $X$ form a base of open neighborhoods of zero in $B(X)$.

Topological spaces $X$ and $Y$ are said to be $M$-equivalent ( $A$-equivalent) if their free (free Abelian) topological groups are topologically isomorphic. We shall say that $X$ and $Y$ are $B$-equivalent if $B(X)$ and $B(Y)$ are topologically isomorphic.

Given $X \supset Y$, we use $B(Y \mid X)$ to denote the topological subgroup of $B(X)$ generated by $Y$.

A special role in the theory of topological groups and in set-theoretic topology is played by Boolean topological groups generated by almost discrete spaces, that is, spaces having only one non-isolated point. With each free filter $\mathcal{F}$ on any set $X$, we associate the almost discrete space $X_{\mathcal{F}}=X \cup\{*\}$ ( $*$ is a point not belonging to $X$ ); all points of $X$ are isolated, and the neighborhoods of $*$ are $\{*\} \cup A, A \in \mathcal{F}$. For a space with infinitely many isolated points, there is no difference between the canonical 
definition of the groups $F(X), A(X)$ and $B(X)$ and Graev's generalizations $F_{G}(X)$, $A_{G}(X)$ and $B_{G}(X)$. Indeed, Graev showed that $F_{G}(X)$ and $A_{G}(X)$ are unique (up to topological isomorphism) and do not depend on the choice of the distinguished point. Graev's argument, which uses only the universality property, carries over word for word to free Boolean topological groups. Thus, when dealing with spaces $X_{\mathcal{F}}$ associated with filters, we can identify $B\left(X_{\mathcal{F}}\right)$ with $B_{G}\left(X_{\mathcal{F}}\right)$ and assume that the only non-isolated point of $X_{\mathcal{F}}$ is the zero of $B\left(X_{\mathcal{F}}\right)$; the descriptions of the neighborhoods of zero and the Graev seminorm are altered accordingly. To understand how they change, take the new (but in fact, the same) space $\widetilde{X}_{\mathcal{F}}=X_{\mathcal{F}} \cup\{0\}$, where 0 is one more isolated point, represent $B\left(X_{\mathcal{F}}\right)$ as the Graev free Boolean topological group $B_{G}\left(\widetilde{X}_{\mathcal{F}}\right)$ with distinguished point (zero of $B_{G}\left(\widetilde{X}_{\mathcal{F}}\right)$ ) 0 , and consider the topological isomorphism $g \mapsto g+0$ between this group and the similar group with distinguished point (zero) *.

For example, since any open cover of $X_{\mathcal{F}}$ can be assumed to consist of a neighborhood of $*$ and singletons, Description II reads as follows in this case: For each $n \in \mathbb{N}$, we fix an arbitrary neighborhood $V_{n}$ of $*$, that is, $A_{n} \cup\{*\}$, where $A_{n} \in \mathcal{F}$, and set:

$$
\begin{gathered}
W=\left\{V_{n}\right\}_{n \in \mathbb{N}}, \\
U\left(V_{n}\right)=\left\{x+*: x \in V_{n}\right\}=\left\{x: x \in A_{n}\right\} \quad(* \text { is zero }), \\
U(W)=\bigcup_{n \in \mathbb{N}}\left(U\left(V_{1}\right)+U\left(V_{2}\right)+\cdots+U\left(V_{n}\right)\right)=\bigcup_{n \in \mathbb{N}}\left\{x_{1}+\cdots+x_{n}: x_{i} \in A_{i} \text { for } i \leq n\right\} .
\end{gathered}
$$

The sets $U(W)$, where the $W$ range over all sequences of neighborhoods of $*$, form a neighborhood base at zero for the topology of $B\left(X_{\mathcal{F}}\right)$. Strictly speaking, to obtain a full analogy with Description II of the Markov free group topology, we should set:

$$
U(W)=\bigcup_{n \in \mathbb{N}}\left(2 U\left(V_{1}\right)+2 U\left(V_{2}\right)+\cdots+2 U\left(V_{n}\right)\right)=\bigcup_{n \in \mathbb{N}}\left\{x_{1}+y_{1} \cdots+x_{n}+y_{n}: x_{i}, y_{i} \in V_{i} \text { for } i \leq n\right\},
$$

but this would not affect the topology: the former $U(W)$ equals the latter for a sequence of smaller neighborhoods, say $V_{n}^{\prime}=\bigcap_{i \leq 2 n} V_{i}$ (remember that some of the $x_{i}$ and $y_{i}$ in the expression for $U(W)$ may equal $*$, that is, vanish).

Similarly, the base neighborhoods of zero in Description III take the form:

$$
U(d)=\left\{x_{1}+x_{2}+\cdots+x_{n}: n \in \mathbb{N}, x_{1}, \ldots, x_{n} \in X, \sum_{i=1}^{n} d\left(x_{i}, *\right)<1\right\}
$$


where $d$ ranges over continuous pseudometrics on $X_{\mathcal{F}}$ (again, we should set $U(d)=$ $\left\{x_{1}+y_{1}+x_{2}+y_{2}+\cdots+x_{n}+y_{n}: n \in \mathbb{N}, x_{i}, y_{i} \in X \cup\{*\}, \sum_{i=1}^{n} d\left(x_{i}, y_{i}\right)<1\right\}$, but this would not make any difference).

It is also easy to see that the isomorphism between $B_{G}\left(\widetilde{X}_{\mathcal{F}}\right)$ (with distinguished point $*$ ) and $B\left(X_{\mathcal{F}}\right)$ does not essentially affect the sets of words of length at most $n$; in particular, they remain closed, and $B_{G}\left(\widetilde{X}_{\mathcal{F}}\right)$ is the inductive limit of these sets with the induced topology if and only if $B\left(X_{\mathcal{F}}\right)$ has the inductive limit topology. In what follows, by $B\left(X_{\mathcal{F}}\right)$, we shall usually mean the Graev free Boolean topological group with zero $*$.

Thus, $B\left(X_{\mathcal{F}}\right)$ is naturally identified with the group $[X]^{<\omega}$ of all finite subsets of $X$ under the operation $\triangle$ of symmetric difference $(A \triangle B=(A \backslash B) \cup(B \backslash A))$. The point $*$, which is the zero element of $B\left(X_{\mathcal{F}}\right)$, is identified with the empty set $\varnothing$, which belongs to $[X]^{<\omega}$ as the zero element. In the context of free Boolean groups on almost discrete spaces, we also identify each $x \in X$ with the one-point set $\{x\} \in[X]^{<\omega}$.

Sets of the form $[X]^{<\omega}$ often arise in set-theoretic topology and in forcing. The role of $X$ is often played by $\omega$, and the filter $\mathcal{F}$ is usually an ultrafilter with certain properties.

We assume all filters $\mathcal{F}$ on $\omega$ to be free, i.e., to contain the Fréchet filter (of all cofinite sets).

A filter $\mathcal{F}$ on $\omega$ is said to be a $P$-filter if, for any family of $A_{i} \in \mathcal{F}, i \in \omega$, the filter $\mathcal{F}$ contains a pseudo-intersection of this family, i.e., a set $A \subset \omega$ such that $\left|A \backslash A_{i}\right|<\omega$ for all $i \in \omega$. For ultrafilters, this property is equivalent to being a $P$-point, or weakly selective, ultrafilter. A filter $\mathcal{F}$ on $\omega$ is said to be a Ramsey filter if, for any family of $A_{i} \in \mathcal{F}, i \in \omega$, the filter $\mathcal{F}$ contains a diagonal of this family, i.e., a set $D \subset \omega$ such that, whenever $i, j \in D$ and $i<j$, we have $j \in A_{i}$. Ultrafilters with this property are known as Ramsey, or selective, ultrafilters.

We use the standard notation $[\omega]^{<\omega}$ for the set of all finite subsets of $\omega$ and $\omega^{<\omega}$ for the set of all finite sequences of elements of $\omega$. Given $s, t \in[\omega]<\omega, s \sqsubset t$ means that $s$ is an initial segment of $t$, i.e., $s \subset t$ and all elements of $t \backslash s$ are greater than all elements of $s$. For $s \in[\omega]^{<\omega} \backslash\{\varnothing\}$, by max $s$, we mean the greatest element of $s$ in the ordering of $\omega$. We also set $\max \varnothing=-1$.

\section{A Comparison of Free, Free Abelian and Free Boolean Topological Groups}

\subsection{Similarity}

There are a number of known properties of free and free Abelian topological groups that automatically carry over to free Boolean topological groups simply because they are preserved by taking topological quotient groups or, more generally, by continuous maps. Thus, if $F(X)$ (and $A(X)$ ) is separable, Lindelöf, ccc, and so on, 
then so is $B(X)$. It is also quite obvious that $X$ is discrete if and only if so are $F(X)$, $A(X)$ and $B(X)$.

Let $X$ be a space, and let $Y$ be its subspace. The topological subgroup $B(Y \mid X)$ of $B(X)$ generated by $Y$ is not always the free Boolean topological group on $Y$ (the induced topology of $B(Y \mid X)$ may be weaker). Looking at Description I of the free group topology on $B(X)$, we see that $X$ and $Y$ equipped with the universal uniformities $\mathcal{U}_{X}$ and $\mathcal{U}_{Y}$ are uniform subspaces of $B(X)$ and $B(Y)$ with their group uniformities $\mathcal{W}_{B(X)}$ and $\mathcal{W}_{B(Y)}$ (generated by entourages of the form $W(U)=\{(g, h): h \in g+U\}$, where $U$ ranges over all neighborhoods of zero in the corresponding group), which completely determine the topologies of $B(X)$ and $B(Y)$. Thus, if the topology of $B(Y \mid X)$ coincides with that of $B(Y)$, then, like in the case of free and free Abelian topological groups [28,29], $\left(Y, \mathcal{U}_{Y}\right)$ must be a uniform subspace of $\left(X, \mathcal{U}_{X}\right)$, which means that any bounded continuous pseudometric on $Y$ can be extended to a continuous pseudometric on $X$ (in this case, $Y$ is said to be $P$-embedded in $X$ [30]). The converse has been proven to be true for free Abelian (presented in [28] with an incomplete proof and completely proven in [29]) and even free [27] (see also [22], where a minor misprint in the condition $3^{\circ}$ on p. 186 of [27] is corrected) topological groups. Since $B(X)$ and $B(Y)$ are the topological quotients of $A(X)$ and $A(Y)$ by the subgroups of squares $A(2 X)$ and $A(2 Y)$ and $A(2 Y)=A(2 X) \cap A(Y)$, we immediately obtain the following theorem.

Theorem 4. Let $X$ be a space, and let $Y$ be its subspace. The topological subgroup of the free Boolean groups $B(X)$ generated by $Y$ is the free topological group $B(Y)$ if and only if each bounded continuous pseudometric on $Y$ can be extended to a continuous pseudometric on $X$.

Any space $X$ is closed in its free Boolean topological group $B(X)$ (see, e.g., ([9], Theorems 2.1 and 2.2)), as well as in $F(X)$ and $A(X)$ [1,2]. Moreover, all $F_{n}(X), A_{n}(X)$ and $B_{n}(X)$ (the sets of words of length at most $n$ ) are closed in their respective groups, as well. The most elegant proof of this fact was first proposed by Arkhangel'skii in the unavailable book [31] (for $F(X)$, but the argument works for $A(X)$ and $B(X)$ without any changes): Note that all $F_{n}(\beta X) \subset F(\beta X)$ are compact, since these are the continuous images of $\left(X \oplus\{e\} \oplus X^{-1}\right)^{n}$ under the natural multiplication maps $i_{n}:\left(x_{1}^{\varepsilon_{1}}, \ldots, x_{n}^{\varepsilon_{n}}\right) \mapsto x_{1}^{\varepsilon_{1}} \ldots x_{n}^{\varepsilon_{n}}$ (here, $e$ denotes the identity element of $F(X), \varepsilon_{i}= \pm 1$, and the word $x_{1}^{\varepsilon_{1}} \ldots x_{n}^{\varepsilon_{n}}$ may be reducible, i.e., have length shorter than $\left.n\right)$. Therefore, the $F_{n}(\beta X)$ are closed in $F(\beta X)$, and hence, the sets $F_{n}(X)=F_{n}(\beta X) \cap F(X \mid \beta X)$ are closed in $F(X \mid \beta X)$. It follows that these sets are also closed in $F(X)$, which is the same group as $F(X \mid \beta X)$, but has stronger topology.

The topological structure of a free group becomes much clearer when this group has the inductive limit topology (or, equivalently, when the inductive limit topology is a group topology). The problem of describing all spaces for which $F(X)$ (or 
$A(X))$ possesses this property has proven extremely difficult (and is still unsolved). Apparently, the problem was first stated explicitly by Pestov and Tkachenko in 1985 [32], but it was tackled as early as in 1948 by Graev [3], who proved that the free topological group of a compact space has the inductive limit topology. Then, Mack, Morris and Ordman [33] proved the same for $k_{\omega}$-spaces. The strongest (to the author's knowledge) result in this direction was obtained by Tkachenko [34], who proved that if $X$ is a $P$-space or a $C_{\omega}$-space (the latter means $X$ is the inductive limit of an increasing sequence $\left\{X_{n}\right\}$ of its closed subsets such that all finite powers of each $X_{n}$ are countably compact and strictly collection-wise normal), then $F(X)$ has the inductive limit topology. All of these sufficient conditions are also valid for $A(X)$ and $B(X)$ by virtue of the following simple observation.

Proposition 5. Suppose that $X=\cup_{n \in \mathbb{N}} X_{n}, Y=\cup_{n \in \mathbb{N}} Y_{n}, X$ is the inductive limit of its subspaces $X_{n}, n \in \mathbb{N}$, and $f: X \rightarrow Y$ is a quotient map such that $f\left(X_{n}\right)=Y_{n}$ for each $n \in \mathbb{N}$. Then, $Y$ is the inductive limit of its subspaces $Y_{n}$.

Proof. Let $U \subset Y$ be such that all $U_{n}=U \cap Y_{n}$ are open in $Y_{n}$. Consider $V=f^{-1}(U)$ and $V_{n}=f^{-1}\left(U_{n}\right) \cap X_{n}$ for $n \in \omega$. Each $V_{n}$ is open in $X_{n}$, because the restriction of $f$ to $X_{n}$ is continuous and $f\left(X_{n}\right)=Y_{n}$. On the other hand,

$$
V_{n}=f^{-1}\left(U \cap Y_{n}\right) \cap X_{n}=\left(f^{-1}(U) \cap f^{-1}\left(Y_{n}\right)\right) \cap X_{n}=V \cap X_{n} ;
$$

therefore, $V$ is open in $X$. Since the map $f$ is quotient, it follows that $U=f(V)$ is an open set.

For $X$ of the form $\omega_{\mathcal{F}}$ (where $\mathcal{F}$ is a filter on $\omega$ ), not only the sufficient conditions mentioned above, but also a necessary and sufficient condition for $F(X)$ and $A(X)$ to have the inductive limit topology is known. This condition is also valid for $B(X)$.

Theorem 6. Given a filter $\mathcal{F}$ on $\omega, B\left(\omega_{\mathcal{F}}\right)$ has the inductive limit topology if and only if $\mathcal{F}$ is a P-filter.

Proof. This theorem is true for free and free Abelian topological groups [35]. Therefore, by Proposition 5, $B\left(\omega_{\mathcal{F}}\right)$ has the inductive limit topology for any $P$-filter. It remains to prove that if $B\left(\omega_{\mathcal{F}}\right)$ is the inductive limit of the $B_{n}\left(\omega_{\mathcal{F}}\right)$, then $\mathcal{F}$ is a $P$-filter.

Thus, suppose that $B\left(\omega_{\mathcal{F}}\right)$ is the inductive limit of the $B_{n}\left(\omega_{\mathcal{F}}\right)$, but $\mathcal{F}$ is not a $P$-filter, that is, there exists a decreasing sequence of $A_{n} \in \mathcal{F}, n \in \omega$, such that, for any $A \in \mathcal{F}$, there is an $i$ for which the intersection $A \cap A_{i}$ is infinite. As usual, we assume that the zero element of $B\left(\omega_{\mathcal{F}}\right)$ is the non-isolated point $*$ of $\omega_{\mathcal{F}}$. 
Without loss of generality, we can assume that $A_{0}=\omega$ and all sets $A_{n} \backslash A_{n+1}$ are infinite. We enumerate these sets as:

$$
A_{n} \backslash A_{n+1}=\left\{x_{n i}: i \in \omega\right\}
$$

and put:

$$
D_{n}=\left\{x_{n m}+x_{i_{1} j_{1}}+x_{i_{2} j_{2}}+\cdots+x_{i_{n} j_{n}}: n<i_{1}<i_{2}<\cdots<i_{n}<j_{1}<j_{2}<\cdots<j_{n}<m\right\}
$$

for all $n \in \omega$. Let us show that each $D_{n}$ is a closed discrete subset of $B\left(\omega_{\mathcal{F}}\right)$. Fix $n$ and consider $X=\{*\} \cup\left\{x_{n i}: i \in \omega\right\}$ and the retraction $r: \omega_{\mathcal{F}} \rightarrow X$ that takes $\omega_{\mathcal{F}} \backslash X$ to $\{*\}$. Clearly, $X$ is discrete, and the map $r$ is continuous. Let $\hat{r}: B\left(\omega_{\mathcal{F}}\right) \rightarrow B(X)$ be the homomorphic extension of $r$; then, $\hat{r}$ continuously maps $B\left(\omega_{\mathcal{F}}\right)$ onto the discrete group $B(X)$. For any $g \in B(X)$, the set $\hat{r}^{-1}(g) \cap D_{n}$ is finite: if $\hat{r}^{-1}(g) \cap D_{n}$ is nonempty, then we have $g=\hat{r}\left(x_{n m_{0}}+x_{i_{0_{1}} j_{0_{1}}}+x_{i_{0_{2}} j_{0_{2}}}+\cdots+x_{i_{0_{n}} j_{0_{n}}}\right)$ for some $m_{0}, i_{0_{k}}, j_{0_{k}} \in \omega$ such that $n<i_{0_{1}}<i_{0_{2}}<\cdots<i_{0_{n}}<j_{0_{1}}<j_{0_{2}}<\cdots<j_{0_{n}}<m_{0}$, whence $g=x_{n m_{0}}$ and:

$$
\begin{aligned}
& \hat{r}^{-1}(g) \cap D_{n}=\left\{x_{n m_{0}}+x_{i_{1} j_{1}}+x_{i_{2} j_{2}}+\cdots+x_{i_{n} j_{n}}:\right. \\
& \left.n<i_{1}<i_{2}<\cdots<i_{m}<j_{1}<j_{2}<\cdots<j_{n}<m_{0}\right\} .
\end{aligned}
$$

Since the sets $\hat{r}^{-1}(g), g \in B(X)$, form an open cover of $B\left(\omega_{\mathcal{F}}\right)$, it follows that $D_{n}$ is a closed discrete subspace of $B\left(\omega_{\mathcal{F}}\right)$.

The length of each word in $D_{n}$ equals $n+1$. Therefore, $D=\bigcup_{n} D_{n}$ is closed in the inductive limit topology. It remains to show that $*$ (the zero of $B\left(\omega_{\mathcal{F}}\right)$ ) belongs to the closure of $D$ in the free group topology, i.e., that $U(d) \cap D \neq \varnothing$ for any continuous pseudometric $d$ on $\omega_{\mathcal{F}}$ (see Description III of the topology of $B\left(\omega_{\mathcal{F}}\right)$ ).

Take an arbitrary (continuous) pseudometric $d$ on $\omega_{\mathcal{F}}$. In $\omega_{\mathcal{F}}$, the ball $B_{d}\left(*, \frac{1}{2}\right)$ of radius $\frac{1}{2}$ centered at $*$ with respect to $d$ is a neighborhood of $*$; that is, the punctured ball (with $*$ removed) belongs to $\mathcal{F}$. By assumption, there is an $n \in \omega$ for which the set $\left.M=\left\{i \in \omega: d\left(*, x_{n i}\right)<\frac{1}{2}\right)\right\}$ is infinite. Since $B_{d}\left(*, \frac{1}{2 n}\right) \cap A_{n+1}$ is a punctured neighborhood of $*$ and hence belongs to $\mathcal{F}$, it follows by assumption that the sets $J_{i}=\left\{j \in \omega: d\left(*, x_{i j}\right)<\frac{1}{2 n}\right\}$ are infinite for infinitely many $i$. Choose $i_{1}<i_{2}<\cdots<i_{n}$ greater than $n$ so that all $J_{i_{k}}$ are infinite, then choose $j_{k} \in J_{i_{k}}$, $k \leq n$, so that $i_{n}<j_{1}<\cdots<j_{n}$, and take $m \in M$ such that $m>j_{n}$. We have $g=x_{n m}+x_{i_{1} j_{1}}+x_{i_{2} j_{2}}+\cdots+x_{i_{n} j_{n}} \in D_{n}$. We also have $g \in U(d)$, because:

$$
d\left(*, x_{n m}\right)+\sum_{k=1}^{n} d\left(*, x_{i_{k} j_{k}}\right)<\frac{1}{2}+n \frac{1}{2 n}=1 .
$$


Therefore, $g \in D_{n} \cap U(d)$.

In [36] Tkachuk proved that the free Abelian topological group of a disjoint union of two spaces $X$ and $Y$ is topologically isomorphic to the direct sum $A(X) \oplus A(Y)=A(X) \times A(Y)$. His argument carries over to varieties of Abelian topological groups closed under direct sums (or, in topological terminology, $\sigma$-products with respect to the zero elements of factors) with the box topology. We denote such sums by $\sigma \square$.

Proposition 7. For any family $\left\{X_{\alpha}: \alpha \in A\right\}$ of spaces,

$$
A\left(\bigoplus_{\alpha \in A} X_{\alpha}\right) \cong \sigma \square_{\alpha \in A} A\left(X_{\alpha}\right) \quad \text { and } \quad B\left(\bigoplus_{\alpha \in A} X_{\alpha}\right) \cong \sigma \square_{\alpha \in A} B\left(X_{\alpha}\right) .
$$

If all $X_{\alpha}$ are zero-dimensional, then:

$$
A^{\operatorname{lin}}\left(\bigoplus_{\alpha \in A} X_{\alpha}\right) \cong \sigma \square_{\alpha \in A} A^{\operatorname{lin}}\left(X_{\alpha}\right) \quad \text { and } \quad B^{\operatorname{lin}}\left(\bigoplus_{\alpha \in A} X_{\alpha}\right) \cong \sigma \square_{\alpha \in A} B^{\operatorname{lin}}\left(X_{\alpha}\right) .
$$

Proof. Let $T$ stand for $A, B, A^{\lim }$ or $B^{\lim }$, and let $0_{\alpha}$ denote the zero element of $T\left(X_{\alpha}\right)$. For each $\alpha \in A$, we set $X_{\alpha}^{\prime}=\sigma \square_{\beta \in A} Y_{\beta}$, where $Y_{\alpha}=X_{\alpha}$ and $Y_{\beta}=\left\{0_{\beta}\right\}$ for $\beta \neq \alpha$. Every $X_{\alpha}^{\prime}$ is embedded in the group $T_{\alpha}^{\prime}\left(X_{\alpha}\right)$ defined accordingly as a product of $T\left(X_{\alpha}\right)$ and zeros. Clearly, the union $\bigcup_{\alpha \in A} X_{\alpha}^{\prime}$ algebraically generates $\sigma \square_{\alpha \in A} T\left(X_{\alpha}\right)$ and is homeomorphic to $\bigoplus_{\alpha \in A} X_{\alpha}$. It remains to show that the homomorphic extension of any continuous map of this union to any topological group from the corresponding variety is continuous. Let $f: \bigcup_{\alpha \in A} X_{\alpha}^{\prime} \rightarrow G$ be such a map. For each $\alpha \in A$, the homomorphic extension $\hat{f}_{\alpha}: T_{\alpha}^{\prime}\left(X_{\alpha}\right) \rightarrow G$ of the restriction of $f$ to $X_{\alpha}^{\prime}$ is continuous. We define $\hat{f}: \sigma \square_{\alpha \in A} T\left(X_{\alpha}\right) \rightarrow G$ by setting $\hat{f}\left(\left(g_{\alpha}\right)_{\alpha \in A}\right)=\sum_{\alpha \in A} \hat{f}_{\alpha}\left(g_{\alpha}\right)$ for each $\left(g_{\alpha}\right)_{\alpha \in A} \in \sigma \square_{\alpha \in A} T\left(X_{\alpha}\right)$; the sum is defined, because any element of $\sigma \square_{\alpha \in A} T\left(X_{\alpha}\right)$ has only finitely many nonzero components. Let us show that $\hat{f}$ is continuous. It suffices to check continuity at the zero element of $\sigma \square_{\alpha \in A} T\left(X_{\alpha}\right)$. Take any neighborhood $U$ of zero in $G$. Its preimages $V_{\alpha}$ under the component maps $\hat{f}_{\alpha}$ are open neighborhoods of zero in $T_{\alpha}^{\prime}\left(X_{\alpha}\right)$. The product $\sigma \square_{\alpha} V_{\alpha}$ is the preimage of $U$ under $\hat{f}$, and it is open in the box topology.

The free Boolean topological group of a non-discrete space is never metrizable (as well as the free and free Abelian topological groups). Indeed, if $B(X)$ is metrizable and $X$ is non-discrete, then $X$ contains a convergent sequence $S$ with limit point $*$, and $B(S)=B(S \mid X)$ (see Theorem 4); thus, it suffices to show that $B(S)$ is non-metrizable. Suppose that it is metrizable. Then, the topology of $B(S)$ is generated by a continuous 
norm $\|\cdot\|$. For all pairs of positive integers $n$ and $m \leq n$, choose different $s_{n_{m}} \in S$ so that $\left\|s_{n_{m}}+*\right\|<\frac{1}{n^{2}}$. Clearly, the set:

$$
D=\left\{\left(s_{n_{1}}+*\right)+\left(s_{n_{2}}+*\right)+\ldots\left(s_{n_{n}}+*\right): n \geq 0\right\}
$$

has finite intersection with each $B_{k}(S)$; hence, it must be discrete, because $B(S)$ has the inductive limit topology. On the other hand, $D$ is a sequence convergent to zero, since:

$$
\left\|\left(s_{n_{1}}+*\right)+\left(s_{n_{2}}+*\right)+\ldots\left(s_{n_{n}}+*\right)\right\| \leq \sum_{i=1}^{n}\left(s_{n_{i}}+*\right)<n \cdot \frac{1}{n^{2}}=\frac{1}{n} .
$$

The list of properties shared by free, free Abelian and free Boolean topological groups that can be proven without much effort is very long. Many of these properties are proven for Boolean groups by analogy, but sometimes, their proofs are drastically simplified. We conclude our brief excursion by one such examples. The proof of the following theorem for free topological groups given in [27] is extremely complicated (it is based on a more general construction). The proof given in [22] is much shorter, but still very cumbersome. In the Boolean case, the proof becomes almost trivial.

Theorem 8. If $\operatorname{dim} X=0$, then ind $B(X)=0$.

Proof. Any continuous pseudometric $d$ on $X$ is majorized by a non-Archimedean pseudometric $\rho$ (a pseudometric $\rho$ is said to be non-Archimedean if $\rho(x, z) \leq$ $\max \{\rho(x, y), \rho(y, z)\}$ for any $x, y, z \in X)$ taking only values of the form $\frac{1}{2^{n}}$. To see this, it suffices to consider the elements $V_{0}, V_{1}, \ldots$ of the universal uniformity on $X$ which are determined by decreasing disjoint open refinements $\gamma_{0}, \gamma_{1}, \ldots$ of the covers of $X$ by balls of radii $\frac{1}{2^{1}}, \frac{1}{2^{2}}, \ldots$ with respect to $d$ and apply the construction in the proof of Theorem 8.1.10 of [37] (see also [38]). Since the covers $\gamma_{n}$ determining the entourages $V_{n}$ are disjoint and each $\gamma_{i+1}$ is a refinement of $\gamma_{i}$, it follows that the function $f$ in this construction has the property $f(x, z) \leq \max \{f(x, y), f(y, z)\}$, and therefore, the pseudometric $\rho$ constructed there from $f$ is non-Archimedean and takes the values $\frac{1}{2^{n}}$. Clearly, it majorizes $d$.

Each value $\|g\|_{\rho}, g \in B(X)$, of the Graev extension $\|\cdot\|_{\rho}$ of $\rho$ is either one or a finite sum of values of $d$ (recall that the minimum in the expression for $\|g\|_{\rho}$ is attained at an irreducible representation of $g$ ). Hence, $\|\cdot\|_{\rho}$ takes only rational values, and the balls with irrational radii centered at zero in this norm are open and closed. They form a base of neighborhoods of zero, and their translates form a base of the entire topology on $B(X)$. 


\subsection{Difference}

Pestov gave an example of a space $X$ for which $F(X)$ is not homeomorphic to $A(X)$ [39]. Spaces for which $A(X)$ is not homeomorphic to $B(X)$ exist, too.

Proposition 9. The free Abelian topological group of any connected space has infinitely many connected components. The free Boolean topological group of any connected space has two connected components.

Proof. Consider a connected space $X$. The connected component of zero in $A(X)$ is the subgroup $A^{\mathrm{c}}(X)$ consisting of all words $\sum_{i=1}^{n} x_{i}^{\varepsilon_{i}}$ with $\sum_{i=1}^{n} \varepsilon_{i}=0$ (see ([17], Lemma 7.10.2)). Clearly, all words in this subgroup are of even length, and the canonical homomorphism $A(X) \rightarrow B(X)$ takes $A^{\mathrm{c}}(X)$ to the subgroup $B^{\mathrm{c}}(X)$ of $B(X)$ consisting of all words of even length. Since the canonical homomorphism is continuous and open, the subgroup $B^{\mathrm{C}}(X)$ is connected and open (and hence, closed), and it has index two in $B(X)$. Thus, $B(X)$ has two connected components, while $A(X)$ has infinitely many connected components, because $A(X) / A^{\mathrm{c}}(X) \cong \mathbb{Z}$ (see ([17], Lemma 7.10.2)).

There is a fundamental difference in the very topological-algebraic nature of free, free Abelian and free Boolean groups. Thus, nontrivial free and free Abelian groups admit no compact group topologies (see [40]); this follows from the well-known algebraic description of infinite compact Abelian groups ([41], Theorem 25.25). On the other hand, for any infinite cardinal $\kappa$, the direct sum $\bigoplus_{2^{\kappa}} \mathbb{Z}_{2}$ of $2^{\kappa}$ copies of $\mathbb{Z}_{2}$ (that is, the free Boolean group of rank $2^{\kappa}$ ) is algebraically isomorphic to the Cartesian product $\left(\mathbb{Z}_{2}\right)^{\kappa}([42]$, Lemma 4.5) and, therefore, admits compact group topologies (e.g., the product topology).

The free and free Abelian groups are never finite, while the free Boolean group of any finite set is finite.

The free and free Abelian topological groups of any completely regular Hausdorff topological space $X$ contain all finite powers $X^{n}$ of $X$ as closed subspaces. Thus, each $X^{n}$ is homeomorphic to the closed subset $\left\{x_{1} \ldots x_{n}: x_{i} \in X\right.$ for $\left.i=1 \leq n\right\}$ of $F(X)[43]$ and to the closed subset $\left\{x_{1}+2 x_{2}+\cdots+n x_{n}: x_{i} \in X\right.$ for $\left.i=1 \leq n\right\}$ of $A(X)$ [44]. (Arkhangel'skii announced the result for $F(X)$ in [43] and proved it in [31] by considering the Stone-Čech compactification of $X$ and its free topological group; details can be found in Theorem 7.1.13 of [17]. Unfortunately, the book [31], which is a rotaprint edition of a lecture course, is (and always was) virtually unavailable, even in Russia. Thus, the result was rediscovered by Joiner [45] and the idea of proof by Morris [9] (see also [46]). In fact, both Arkhangel'skii and Joiner proved a stronger statement; namely, they gave the same complete description of the topological 
structure of all $F_{n}(X)$, although obtained by different methods (Arkhangel'skii proof is much shorter).)

However, the situation with free Boolean topological groups is much more complicated. For example, consider extremally disconnected free topological groups.

Extremally disconnected groups are discussed in the next section. Here, we only mention that non-discrete $F(X)$ and $A(X)$ are never extremally disconnected, while $B(X)$ may be non-discrete and extremally disconnected under certain set-theoretic assumptions (e.g., under $\mathrm{CH}$ ), even for countable $X$ of the form $\omega_{\mathcal{F}}$, and that any hereditarily normal, in particular, countable, extremally disconnected space, is hereditarily extremally disconnected (this is shown in the next section). It follows that if $X$ is a non-discrete countable space for which $B(X)$ is extremally disconnected, then $B(X)$ does not contain $X^{2}$ as a subspace. Indeed, otherwise, $X^{2}$ is extremally disconnected (and non-discrete), and the existence of such spaces is prohibited by the following simple observation; it must be known, although the author failed to find a reference.

Proposition 10. If $X \times X$ is extremally disconnected, then $X$ is discrete.

This immediately follows from Frolík's general theorem that the fixed-point set of any surjective self-homeomorphism of an extremally disconnected space is clopen [47]: it suffices to consider the self-homeomorphism of $X \times X$ defined by $(x, y) \mapsto(y, x)$. (Frolík proved this theorem for compact extremally disconnected spaces and not necessarily surjective self-homeomorphisms; in the surjective case, the theorem is extended to non-compact spaces by considering their Stone-Čech compactifications, which are always extremally disconnected for extremally disconnected spaces (this and other fundamental properties of extremally disconnected spaces can be found in the book [48]).)

Thus, there exist (under $\mathrm{CH}$ ) filters $\mathcal{F}$ on $\omega$ for which $\left(\omega_{\mathcal{F}}\right)^{2}$ is not contained in $B\left(\omega_{\mathcal{F}}\right)$ as a subspace. However, in the simplest case where $\mathcal{F}$ is the Fréchet filter (i.e., $\omega_{\mathcal{F}}$ is a convergent sequence), $B\left(\omega_{\mathcal{F}}\right)$ not merely contains $\left(\omega_{\mathcal{F}}\right)^{n}$, but is topologically isomorphic to $B\left(\omega_{\mathcal{F}}\right)^{n}$ for all $n$ by virtue of Proposition 7 and the fact that a convergent sequence is $B$-equivalent to the disjoint union of two convergent sequences, which can be demonstrated as follows.

Any $M$-equivalent spaces are $A$-equivalent, and any $A$-equivalent spaces are $B$-equivalent, because $A(X)(B(X))$ is the quotient of $F(X)(A(X))$ by an algebraically determined subgroup not depending on $X$. Therefore, all known sufficient conditions for $M$ - and $A$-equivalence (see, e.g., [3,4,49-51]) remain valid for $B$-equivalence. In particular, if $X_{0}$ is a space, $K$ is a retract of $X_{0}, X$ is the space obtained by adding an isolated point to $X_{0}$ and $Y=X_{0} / K \oplus K$, then $X$ and $Y$ are $M$-equivalent ([50], Theorem 2.4). This immediately implies the required $B$-equivalence of a convergent 
sequence $S$ and the disjoint union $S \oplus S$ of two convergent sequences: it suffices to take $S \oplus S$ for $X_{0}$ and $X$ and the two-point set of the two limit points in $S \oplus S$ for $K$.

However, there exist $B$-equivalent spaces, which are neither $F$-nor $A$-equivalent. Genze, Gul'ko and Khmyleva obtained necessary and sufficient conditions for infinite initial segments of ordinals to be $F-, A$ - and $B$-equivalent [20] (see also [21]). It turned out that the criteria for $F$ - and $A$-equivalence are the same, and the criterion for $B$-equivalence differs from them; see [20] for details.

Finally, the following theorem shows that there is also a fundamental difference between free groups of the varieties of Abelian and Boolean linear topological groups.

Theorem 11. The free Boolean linear topological group of any strongly zero-dimensional pseudocompact space is precompact.

Proof. Let $X$ be a strongly zero-dimensional pseudocompact space. As mentioned in the preceding section, a base of neighborhoods of zero in $B^{\lim }(X)$ is formed by subgroups of the form:

$$
\langle U(\gamma)\rangle=\left\{\sum_{i=1}^{n}\left(x_{i}+y_{i}\right): n \in \omega,\left(x_{i}, y_{i}\right) \in U_{i} \in \gamma \text { for } i \leq n\right\},
$$

where $\gamma$ in a disjoint open cover of $X$; note that all such covers are finite. Clearly,

$$
\langle U(\gamma)\rangle=\left\{\sum_{i=1}^{2 n} x_{i}: n \in \omega,\left|\left\{i \leq 2 n: x_{i} \in U\right\}\right| \text { is even for each } U \in \gamma\right\} .
$$

Every such subgroup has finite index. Therefore, $B(X)$ is precompact.

This theorem is not true for Abelian groups; moreover, free Abelian linear groups

are never precompact. Indeed, the group $A^{\mathrm{c}}(X)=\left\{\sum_{i=1}^{n} x_{i}^{\varepsilon_{i}}: n \in \mathbb{N}, \sum_{i=1}^{n} \varepsilon_{i}=1\right\}$ considered above is always open, being the preimage of the isolated point zero under the homomorphism $A(X) \rightarrow \mathbb{Z}_{2}=\{0,1\}$, which extends the constant map $X \rightarrow\{0,1\}$ taking everything to one. As already mentioned, $A^{\mathrm{c}}(X)$ has infinite index in $A(X)$.

\section{Extremally Disconnected Groups}

There is an old problem of Arkhangel'skii on the existence in ZFC of a non-discrete Hausdorff extremally disconnected topological group; it was posed in 1967 [52] and has been extensively studied since then. The problem is still open even for countable groups, although several consistent examples have been constructed [53-58]. An impression of the state-of-the-art in this area can be gained from Zelenyuk's book [59] and the author's papers [60] and [61]. The 
most recent result (presented in [61]) asserts that, under additional set-theoretic assumptions, a countable extremally disconnected group cannot contain a sequence of open subgroups whose intersection has an empty interior; in other words, if there exists in ZFC a non-discrete countable extremally disconnected group, then there must exist such a group without open subgroups (note in this connection that any extremally disconnected space is strongly zero-dimensional, and any zero-dimensional free Boolean topological group contains a family of open subgroups with trivial intersection (see Theorem 2)). Here, we present a new observation closely related to free Boolean topological groups.

A space $X$ is said to be extremally disconnected if the closure of each open set in this space is open or, equivalently, if any two disjoint open sets have disjoint closures. In particular, the space $X_{\mathcal{F}}$ associated with a filter $\mathcal{F}$ is extremally disconnected if and only if $\mathcal{F}$ is an ultrafilter. The most fundamental properties of extremally disconnected spaces can be found in the book [48]. Much useful information (especially in the topological-algebraic context) is contained in [62]. The central place in the theory of extremally disconnected topological groups is occupied by Boolean topological groups because of the following theorem of Malykhin.

Theorem 12 (Malykhin [54]). Any extremally disconnected group contains an open (and therefore closed) Boolean subgroup.

This theorem follows from Frolík's fixed-point theorem mentioned at the end of the preceding section. In [54], Malykhin reproved Frolík's theorem for the particular self-homeomorphism $g \mapsto g^{-1}$; its fixed-point set $U$ is an open neighborhood of the identity element, and the subgroup generated by an open neighborhood $V$ of the identity for which $V^{2} \subset U$ is as required.

Thus, in the theory of extremally disconnected groups, only Boolean groups matter. As is known, the existence of a non-discrete extremally disconnected free Boolean topological group implies the existence of either measurable cardinals or Ramsey ultrafilters [60] (this is proven by reduction to the free Boolean topological group on a countable space with one non-isolated point); it is also known that the simultaneous nonexistence of measurable cardinals and Ramsey ultrafilters is consistent with ZFC (see [63]). The following two theorems have a stronger consequence.

Theorem 13. Any hereditarily normal extremally disconnected space is hereditarily extremally disconnected.

Proof. Let $X$ be a hereditarily normal extremally disconnected space. We must prove that any $Y \subset X$ is extremally disconnected. We can assume that $Y$ is closed in $X$, because, obviously, any dense subspace of an extremally disconnected space is 
extremally disconnected. We must show that the closures in $Y$ of any disjoint sets $U$ and $V$ which are open in $Y$ are disjoint. Note that such sets $U$ and $V$ are separated (in $Y$ and, therefore, in $X$ ), that is, $\bar{U} \cap V=U \cap \bar{V}=\varnothing$. Since $X$ is hereditarily normal, there exist disjoint open (in $X$ ) sets $U^{\prime} \supset U$ and $V^{\prime} \supset V$ ([37], Theorem 2.1.7). Their closures in $X$ cannot intersect, because $X$ is extremally disconnected; thus, the closures in $Y$ of the smaller sets $U$ and $V$ do not intersect either.

Theorem 14. If $G$ is a hereditarily normal extremally disconnected Boolean group, then any closed linearly independent subset of $G$ contains at most one non-isolated point.

Proof. Let $A \subset G$ be a closed linearly-independent subset of $G$. Suppose that $a \in A$ and $b \in A$ are distinct limit points of $A$. Take their disjoint closed neighborhoods $U \ni a$ and $V \ni b$. Since $A$ is linearly independent and closed, it follows that $a+(V \cap A) \cap b+(U \cap A)=\{a+b\}$, and the sets $a+(V \cap A)$ and $b+(U \cap A)$ are closed. Therefore, the sets $a+((V \backslash\{b\}) \cap A)$ and $b+((U \backslash\{a\}) \cap A)$ are closed in the normal subspace $(a+(V \cap A) \cup b+(U \cap A)) \backslash\{a+b\}$ of $G$ and, hence, can be separated by disjoint open neighborhoods in this subspace. These neighborhoods remain open in $a+(V \cap A) \cup b+(U \cap A)$; obviously, $a+b$ belongs to the closure of each of them, which contradicts the hereditary extremal disconnectedness of $G$.

Corollary 15. If $X$ is a non-discrete countable space for which $B(X)$ is extremally disconnected, then $\mathrm{X}$ is almost discrete.

We shall see in the next section that, in fact, the space $X$ in Corollary 15 must be associated with a Ramsey ultrafilter.

\section{Free Boolean Groups on Filters on $\omega$}

We have already seen in the preceding sections that free Boolean groups on almost discrete countable spaces (associated with filters on $\omega$ ) exhibit interesting behavior. Moreover, they are encountered more often than it may seem at first glance.

Consider any Boolean group $B(X)$ with countable basis $X$. As mentioned in Section 2, this group is (algebraically) isomorphic to the direct sum (or, in topological terminology, $\sigma$-product) $\oplus^{\aleph_{0}} \mathbb{Z}_{2}$ of countably many copies of $\mathbb{Z}_{2}$. There is a familiar natural topology on this $\sigma$-product, namely the usual product topology; let us denote it by $\tau_{\text {prod. }}$. This topology induces the topology of a convergent sequence on $X \oplus\{0\}$ (where 0 denotes the zero element of $B(X))$ and is metrizable; therefore, it never coincides with the topology $\tau_{\text {free }}$ of the free Boolean topological group on $X$. Moreover, $\tau_{\text {prod }}$ is contained in $\tau_{\text {free }}$ only when $X$ is discrete or has the form $\omega_{\mathcal{F}}$ for some filter (recall that we assume all filters to be free, i.e., contain the filter of cofinite sets, and identify the non-isolated points of the associated spaces with the zeros of their free Boolean 
groups). On the other hand, any countable space is zero-dimensional; therefore, any countable free Boolean topological group contains a sequence of subgroups with trivial intersection (see Theorem 2). In [61], the following lemma was proven.

Lemma 16 ([61]). Let $G$ be a countable non-discrete Boolean topological group that contains a family of open subgroups with trivial intersection. Then, there exists a basis of $G$ such that the isomorphism $G \rightarrow \oplus^{\aleph_{0}} \mathbb{Z}_{2}$ taking this basis to the canonical basis of $\oplus^{\aleph_{0}} \mathbb{Z}_{2}$ is continuous with respect to the product topology on $\oplus^{\aleph_{0}} \mathbb{Z}_{2}=\sigma\left(\mathbb{Z}_{2}\right)^{\aleph_{0}}$.

This immediately implies the following assertion.

Theorem 17. Any countable Boolean topological group containing a family of open subgroups with trivial intersection (in particular, any free Boolean topological or linear topological group on a countable space) has either a discrete closed basis or a closed basis homeomorphic to the space $\omega_{\mathcal{F}}$ associated with a filter $\mathcal{F}$ on $\omega$.

Spaces of the form $\omega_{\mathcal{F}}$ are one of the rare examples where the free Boolean topological group is naturally embedded in the free and free Abelian topological groups as a closed subspace. The embedding of $B\left(\omega_{\mathcal{F}}\right)$ into $A\left(\omega_{\mathcal{F}}\right)$ is defined simply by $x_{1}+x_{2}+\cdots+x_{n} \mapsto x_{1}+x_{2}+\cdots+x_{n}$ (for the Graev free groups, which are the same as Markov ones for such spaces), and the embedding into $F\left(\omega_{\mathcal{F}}\right)$ is $x_{1}+x_{2}+$ $\cdots+x_{n} \mapsto x_{1} x_{2} \ldots x_{n}$, provided that $x_{1}<x_{2}<\cdots<x_{n}$. These embeddings take $B\left(\omega_{\mathcal{F}}\right)$ to:

$$
A=\left\{x_{1}+x_{2}+\cdots+x_{n}=\left(x_{1}-*\right)+\left(x_{2}-*\right)+\cdots+\left(x_{n}-*\right): n \in \mathbb{N}, x_{i} \in \omega\right\} \subset A\left(\omega_{\mathcal{F}}\right)
$$

and:

$F=\left\{x_{1} x_{2} \ldots x_{n}=x_{1} *^{-1} x_{2} *^{-1} \ldots x_{n} *^{-1}: n \in \mathbb{N}, x_{i} \in \omega, x_{1}<x_{2}<\cdots<x_{n}\right\} \subset F\left(\omega_{\mathcal{F}}\right)$.

The topologies induced on $A$ and $F$ by $A\left(\omega_{\mathcal{F}}\right)$ and $F\left(\omega_{\mathcal{F}}\right)$ are easy to describe; the restrictions of base neighborhoods of the zero (identity) element to these sets are determined by sequences of open covers of $\omega_{\mathcal{F}}$ (i.e., of neighborhoods of the non-isolated point $*$ ) in the same manner as in Description II (see [22]). A straightforward verification shows that $A, B$ and $B\left(\omega_{\mathcal{F}}\right)$ are homeomorphic. The rigorous proof of this fact is rather tedious, and we omit it.

As mentioned in the Introduction, for any filter $\mathcal{F}$, the free Boolean group on $\omega_{\mathcal{F}}$ is simply $[\omega]^{<\omega}$. Any topology on $[\omega]^{<\omega}$ (as well as on any other set) is a partially ordered (by inclusion) family of subsets. Partial orderings of subsets of $[\omega]^{<\omega}$ have been extensively studied in forcing, and countable Boolean topological groups turn out to be closely related to them. In this section, we shall try to give an intuitive 
explanation of this relationship. The basic definitions and facts related to forcing can be found in Jech's book [64].

By a notion of forcing, we mean a partially ordered set (briefly, poset) $(\mathbb{P}, \leq)$. Elements of a notion of forcing are called conditions; given two conditions $p, q \in \mathbb{P}$, we say that $p$ is stronger than $q$ if $p \leq q$. A partially ordered set $(\mathbb{P}, \leq)$ is separative if, whenever $p \not \leq q$, there exists an $r \leq p$ which is incompatible with $q$. Thus, any topology is a generally non-separative notion of forcing, and the family of all regular open sets in a topology is a separative notion of forcing. Any separative forcing notion $(P, \leq)$ is isomorphic to a dense subset of a complete Boolean algebra. Indeed, consider the set $\mathbb{P} \downarrow p=\{q: q \leq p\}$ for each $p \in \mathbb{P}$. The family $\{X \subset \mathbb{P}$ : $(\mathbb{P} \downarrow p) \subset X$ for every $p \in X\}$ generates a topology on $\mathbb{P}$. The complete Boolean algebra mentioned above is the algebra $\mathrm{RO}(\mathbb{P})$ of regular open sets in this topology.

Two notions of forcing $\mathbb{P}$ and $\mathbb{Q}$ are said to be forcing equivalent if the algebras $\mathrm{RO}(\mathbb{P})$ and $\mathrm{RO}(\mathbb{Q})$ are isomorphic or, equivalently, if $\mathbb{P}$ can be densely embedded in $\mathbb{Q}$ and vice versa (which means that $\mathbb{P}$ and $\mathbb{Q}$ give the same generic extensions).

Roughly speaking, given a countable transitive model $\mathbf{M}$ of set theory, the method of forcing extends $\mathbf{M}$ by adding a so-called generic subset (called also a generic filter) $G$ of $\mathbb{P}$ not belonging to $\mathbf{M}$; the extended model, called a generic extension of $\mathbf{M}$, contains $\bigcup G$, which has certain desired properties ensured by the choice of $\mathbb{P}$ and $G$.

In the context of free Boolean groups on filters, most interesting are two well-known notions of forcing, Mathias forcing and Laver forcing relativized to (usual) filters on $\omega$.

In Mathias forcing relative to a filter $\mathcal{F}$, the forcing poset, denoted $\mathbb{M}(\mathcal{F})$, is formed by pairs $(s, A)$ consisting of a finite set $s \subset \omega$ and an (infinite) set $A \in \mathcal{F}$ such that every element of $s$ is less than every element of $A$ in the ordering of $\omega$. A condition $(t, B)$ is stronger than $(s, A)((t, B) \leq(s, A))$ if $s \sqsubset t, B \subset A$ and $t \backslash s \subset A$.

The poset in Laver forcing consists of subsets of the set $\omega^{<\omega}$ of ordered finite sequences in $\omega$. However, it is more convenient for our purposes to consider its modification consisting of subsets of $[\omega]<\omega$. Thus, we restrict the Laver forcing poset to the set $\omega^{\uparrow<\omega}$ of strictly increasing finite sequences in $\omega$ (this restricted poset is forcing equivalent to the original one) and note that the latter is naturally identified with $[\omega]^{<\omega}$. Below, we give the definition of the corresponding modification of Laver forcing.

The definition of Laver forcing uses the notion of a Laver tree. A Laver tree is a set $p$ of finite subsets of $\omega$ such that:

(i) $p$ is a tree (i.e., if $t \in p$, then $p$ contains any initial segment of $t$ ),

(ii) $p$ has a stem, i.e., a maximal node $s(p) \in p$, such that $s(p) \sqsubset t$ or $t \sqsubset s(p)$ for all $t \in p$ and 
(iii) if $t \in p$ and $s(p) \sqsubset t$, then the set $\operatorname{succ}(t)=\{n \in \omega: n>\max t, t \cup\{n\} \in p\}$ is infinite.

In Laver forcing relative to $\mathcal{F}$, the poset, denoted $\mathbb{L}(\mathcal{F})$, is the set of Laver trees $p$ such that $\operatorname{succ}(t) \in \mathcal{F}$ for any $t \in p$ with $s(p) \sqsubset t$, ordered by inclusion.

The Mathias and Laver forcings $\mathbb{M}(\mathcal{F})$ and $\mathbb{L}(\mathcal{F})$ have the special feature that they diagonalize the filter $\mathcal{F}$ (i.e., add its pseudo-intersection). They determine two natural topologies on $[\omega]^{<\omega}$ : the Mathias topology $\tau_{M}$ generated by the base:

$$
\left\{[s, A]: s \in[\omega]^{<\omega}, A \in \mathcal{F}\right\}, \quad \text { where } \quad[s, A]=\left\{t \in[\omega]^{<\omega}: s \sqsubset t, t \backslash s \subset A\right\} \text {, }
$$

and the Laver topology $\tau_{L}$ generated by all sets $U \subset[\omega]^{<\omega}$ such that:

$$
t \in U \Longrightarrow\{n>\max t: t \cup\{n\} \in U\} \in \mathcal{F} \text {. }
$$

It is easy to see that the Mathias topology is nothing but the topology of the free Boolean linear topological group on $\omega_{\mathcal{F}}$ (recall that linear groups are those with topology generated by subgroups): a base of neighborhoods of zero is formed by the sets $[\varnothing, A]$ with $A \in \mathcal{F}$, that is, by all subgroups generated by elements of $\mathcal{F}$.

The neighborhoods of zero in the Laver topology are not so easy to describe explicitly; their recursive definition immediately follows from that given above for general open sets (the only condition that must be added is $\varnothing \in U$ ). Thus, $U$ is an open neighborhood of zero if, first, $\varnothing \in U$; by definition, $U$ must also contain all $n \in A(\varnothing)$ for some $A(\varnothing) \in \mathcal{F}$ (moreover, $U$ may contain no other elements of size one); for each of these $n$, there must exist an $A(n) \in \mathcal{F}$ such that $A(n) \cap\{0,1, \ldots, n\}=\varnothing$ and $U$ contains all $\{n, m\}$ with $m \in A(n)$ (moreover, $U$ may contain no other element of size two); for any such $\{n, m\}(m>n)$, there must exist an $A(\{n, m\}) \in \mathcal{F}$ such that $A(\{n, m\}) \cap\{0,1, \ldots, m\}=\varnothing$ and $U$ contains all $\{n, m, l\}$ with $l \in A(\{n, m\})$, and so on. Thus, each neighborhood of zero is determined by a family $\left\{A(s): s \in[\omega]^{<\omega}\right\}$ of elements of $\mathcal{F}$. Clearly, the topology $\tau_{L}$ is invariant with respect to translation by elements of $[\omega]^{<\omega}$; upon a little reflection, it becomes clear that $\tau_{L}$ is the maximal invariant topology on $[\omega]^{<\omega}$ in which the filter $\mathcal{F}$ converges to zero. (An invariant topology is a topology with respect to which the group operation is separately continuous; groups with an invariant topology are said to be semi-topological. The convergence of $\mathcal{F}$ to zero means that $\tau_{L}$ induces the initially given topology on $\omega_{\mathcal{F}}$.) Since the free group topology is invariant as well, it is weaker than $\tau_{L}$.

The Mathias topology is, so to speak, the uniform version of the Laver topology: a neighborhood of zero in the Laver topology determined by a family $\{A(s) \in \mathcal{F}: s \in$ $\left.[\omega]^{<\omega}\right\}$ is open in the Mathias topology if and only if there exists a single $A \in \mathcal{F}$ such that $A(s)=A \backslash\{0,1, \ldots, \max s\}$ for each $s$. (In [65], the corresponding relationship 
between Mathias and Laver forcings was discussed from a purely set-theoretic point of view.) Hence, $\tau_{M} \subset \tau_{L}$.

The topology of the free Boolean topological group on $\omega_{\mathcal{F}}$ occupies an intermediate position between the Mathias and the Laver topology: it is not so uniform as the former, but more uniform than the latter. A neighborhood of zero is determined not by a single element of the filter (like in the Mathias topology), but by a family of elements of $\mathcal{F}$ assigned to $s \in[\omega]^{<\omega}$ (like in the Laver topology), but these elements depend only on the lengths of $s$.

The following theorem shows that the Laver topology is a group topology only for special filters. This theorem was proven in 2007 by Egbert Thümmel, who kindly communicated it, together with a complete proof, to the author. The symbols $\tau_{\text {free }}$ and $\tau_{\text {indlim }}$ in its statement denote the topology of the free topological group $B\left(\omega_{\mathcal{F}}\right)$ and the inductive limit topology of $B\left(\omega_{\mathcal{F}}\right)$, respectively.

Theorem 18 (Thümmel, 2007 [66]). For any filter on $\omega$, the following conditions are equivalent:

(i) $\mathcal{F}$ is Ramsey;

(ii) $\tau_{M}=\tau_{\text {free }}=\tau_{\text {indlim }}=\tau_{L}$;

(iii) $\tau_{L}$ is a group topology;

(iv) for any sequence of $A_{i} \in \mathcal{F}, i \in \omega$, the set $U=\{\varnothing\} \cup \bigcup_{i \in \omega}\left[i, A_{i}\right]$ is open in $\tau_{\text {free. }}$.

This theorem is particularly interesting because its original (Thümmel's) proof uses an argument that is simple and still quite typical of the method of forcing. The proof given below only slightly differs from Thümmel's and uses this argument, as well.

Proof. First, note that $\tau_{M} \subset \tau_{\text {free }} \subset \tau_{\text {indlim }} \subset \tau_{L}$. Indeed, the first two inclusions are obvious, and the third one follows from Proposition 3 (or from the inclusion $\tau_{\text {free }} \subset \tau_{L}$ noted above) and the observation that $\tau_{L}$ is the inductive limit of its restrictions to $B_{n}\left(\omega_{\mathcal{F}}\right)$.

Thus, to prove the implication (i) $\Rightarrow$ (ii), it suffices to show that $\tau_{M}=\tau_{L}$ for any Ramsey filter. Let $U$ be a neighborhood of $\varnothing$ in $\tau_{L}$. For each $i \in \omega$, we set:

$$
A_{i}=\bigcap\{\{n>\max s: s \cup\{n\} \in U\}: s \in U, \max s \leq i\} .
$$

Since the number of $s \in[\omega]^{<\omega}$ with $\max s \leq i$ is finite, it follows that $A_{i} \in \mathcal{F}$. Take a diagonal $D \in \mathcal{F}$ for the family $\left\{A_{i}: i \in \omega\right\}$. We can assume that $D \subset A_{0}$. Clearly, $[\varnothing, D] \subset U$, whence $U \in \tau_{M}$.

The implication (ii) $\Rightarrow$ (iii) is trivial.

Let us prove (iii) $\Rightarrow$ (iv). Note that it follows from (iii) that $\tau_{\text {free }}=\tau_{L}$, because $\tau_{\text {free }} \subset \tau_{L}$ and $\tau_{\text {free }}$ is the strongest group topology inducing the initially given 
topology on $\omega_{\mathcal{F}}$. It remains to note that any set of the form $\{\varnothing\} \cup \cup_{i \in \omega}\left[i, A_{i}\right]$, where $A_{i} \in \mathcal{F}$, is open in $\tau_{L}$.

We proceed to the last implication (iv) $\Rightarrow$ (i). Take any family $\left\{A_{i}: i \in \omega\right\} \subset \mathcal{F}$ and consider the set $U$ defined as in (iv). Since this is an open neighborhood of zero in the group topology $\tau_{\text {free, }}$ there exists an open neighborhood $V$ of zero (in $\tau_{\text {free }}$ ) such that $V+V \subset U$. The set $D=\{i \in \omega: i \in V\}$ belongs to $\mathcal{F}$ (because $\tau_{\text {free }}$ induces the initially given topology on $\left.\omega_{\mathcal{F}}\right)$ and is a diagonal of $\left\{A_{i}: i \in \omega\right\}$.

This theorem is worth comparing to Judah and Shelah's proof that if $\mathcal{F}$ is a Ramsey ultrafilter, then $\mathbb{M}(\mathcal{F})$ is forcing equivalent to $\mathbb{L}(\mathcal{F})$ ([67], Theorem 1.20 (i)).

Thümmel also obtained the following remarkable result as a simple corollary of Theorem 18.

Theorem 19 (Thümmel, 2007 [66]). Given a filter $\mathcal{F}$ on $\omega$, the group $B\left(\omega_{\mathcal{F}}\right)$ is extremally disconnected if and only if $\mathcal{F}$ is a Ramsey ultrafilter.

Proof. The proof of the if part is essentially contained in Sirota's construction of a (consistent) example of an extremally disconnected group [56]. The proof of the only if part is based on the equivalence (iv) $\Leftrightarrow$ (i) of Theorem 18: for any family $\left\{A_{i}: i \in \omega\right\} \subset \mathcal{F}$, the set $\bigcup_{i \in \omega}\left[i, A_{i}\right]$ is open even in the Mathias topology, and its closure in $\tau_{\text {free, }}$ which must be open by virtue of extremal disconnectedness, is $\{\varnothing\} \cup \bigcup_{i \in \omega}\left[i, A_{i}\right]$. The assertion (iv) $\Leftrightarrow$ (i) implies that $\mathcal{F}$ is a Ramsey filter. It remains to apply Theorem 13 and recall that $\omega_{\mathcal{F}}$ is extremally disconnected if and only if $\mathcal{F}$ is an ultrafilter.

Thümmel has never published these results, and Theorem 19 was rediscovered by Zelenyuk, who included it, among other impressive results, in his book [59] (see Theorem 5.1 in [59]).

Combining Theorem 19 with Corollary 15, we obtain yet another corollary.

Corollary 20. The free Boolean group on a non-discrete countable space $X$ is extremally disconnected if and only if $X$ is an almost discrete space associated with a Ramsey ultrafilter.

Free Boolean topological and free Boolean linear (that is, Mathias) topological groups on spaces associated with filters, as well as Boolean groups with other topologies determined by filters, are the main tool in the study of topological groups with extreme topological properties (see [59] and the references therein). However, free Boolean (linear) topological groups on filters arise also in more "conservative" domains. We conclude with mentioning an instance of this kind.

The most elegant (in the author's opinion) example of a countable non-metrizable Fréchet-Urysohn group was constructed by Nyikos in [68] under the 
relatively mild assumption $\mathfrak{p}=\mathfrak{b}$ (Hrušák and Ramos-García have recently proven that such an example cannot be constructed in ZFC [69]).

It is clear from general considerations that test spaces most convenient for studying convergence properties that can be defined pointwise (such as the Fréchet-Urysohn property and the related $\alpha_{i}$-properties) are countable almost discrete spaces (that is, spaces of the form $\omega_{\mathcal{F}}$ ), and the most convenient test groups for studying such properties in topological groups are those generated by such spaces, simplest among which are free Boolean linear topological groups. Thus, it is quite natural that Nyikos' example is $B^{\operatorname{lin}}\left(\omega_{\mathcal{F}}\right)$ for a very cleverly constructed filter $\mathcal{F}$. In fact, he constructed it on $\omega \times \omega$ (which does not make any difference, of course) as the set of neighborhoods of the only non-isolated point in a $\Psi$-like space defined by using graphs of functions $\omega \rightarrow \omega$ from a special family. In the same paper, Nyikos proved many interesting convergence properties of groups $B^{\operatorname{lin}}\left(\omega_{\mathcal{F}}\right)$ for arbitrary filters $\mathcal{F}$ on $\omega$. We do not give any more details here: the interested reader will gain much more benefit and pleasure from reading Nyikos' original paper.

\section{A Few Open Problems}

Free Boolean topological groups have not yet been extensively studied, and related unsolved problems are numerous. Some of the problems most interesting to the author are suggested below.

Problem 1. Describe those spaces $X$ whose finite powers are embedded in the free Boolean topological groups $B(X)$. Is it true that if $\mathcal{F}$ is a free ultrafilter on $\omega$, then $\omega_{\mathcal{F}} \times \omega_{\mathcal{F}}$ cannot be embedded in $B\left(\omega_{\mathcal{F}}\right)$ ?

The following problem is open not only for free Boolean topological groups, but also for free and free Abelian ones.

Problem 2. Describe those spaces $X$ for which $B(X)(F(X), A(X))$ is normal.

Of course, if $F(X)$ or $A(X)$ is normal, then so are all finite powers of $X$, because they are embedded in $F(X)$ and $A(X)$ as closed subspaces. However, in the Boolean case, even this has not been proven.

Problem 3. Does there exist a space $X$ such that $B(X)$ is normal, but $X^{2}$ is not?

Similarly, for Boolean groups, the following problem becomes nontrivial.

Problem 4. Describe spaces $X$ for which $B(X)$ is Lindelöf. Does there exist a space $X$ such that $B(X)$ is Lindelöf, but $X^{2}$ is not? 
Problem 5. Does there exist a space $X$ for which $B(X)$ is normal (Lindelöf, ccc), but $F(X)$ or $A(X)$ is not?

Problem 6. Is it true that $B(X)$ is Weil complete for any Dieudonné complete space $X$ ?

A positive answer to this question in the case where $X$ is a product of metrizable spaces would imply a positive answer in the general case. Indeed, any Dieudonné complete space $X$ can be embedded in a product $P$ of metric spaces as a closed subspace in such a way that every bounded continuous pseudometric on $X$ can be extended to $P$. Therefore, by Theorem $4, B(X)$ is a subgroup of $B(P)$; it is easy to see that $B(X)$ is closed in $B(P)$, and hence, $B(X)$ is Weil complete if so is $B(P)$. For free and free Abelian topological groups, Problem 6 has been completely solved: Tkachenko proved that if $X$ is Dieudonné complete, then $A(X)$ is Weil complete [28]; Uspenskii proved the Weil completeness of $F(X)$ in the case where $X$ is a product of metrizable spaces [29]; and the author extended Uspenskii's result to arbitrary Dieudonné complete spaces [27].

The following problem has been solved only for free Abelian topological groups [70].

Problem 7. Is it true that the free (Boolean) topological group of any stratifiable space is stratifiable?

The following two problems have been extensively studied and proven very difficult for free and free Abelian topological groups. Results related to the inductive limit topology were mentioned in Section 3.1, and results related to the natural multiplication maps being quotient can be found, e.g., in [25,34,71-73].

Problem 8. Describe spaces $X$ for which $B(X)$ has the inductive limit topology.

Problem 9. Describe spaces $X$ for which all (or some) of the natural addition maps $i_{n}: X \cup X^{-1} \rightarrow B(X)$ defined by $\left(x_{1}^{\varepsilon_{1}}, x_{2}^{\varepsilon_{2}}, \ldots, x_{n}^{\varepsilon_{n}}\right) \mapsto x_{1}+x_{2}+\cdots+x_{n}$ for $n \in \mathbb{N}$, $x_{i} \in X$ and $\varepsilon_{i}= \pm 1(i \leq n)$ are quotient.

We conclude this short list of problems with a problem closely related to extremally disconnected groups.

Problem 10. Does there exist a (countable) non-discrete Boolean topological group in which all linearly independent sets are closed and discrete? 
Acknowledgments: The author is very grateful to Evgenii Reznichenko for useful discussions and to the referees for their detailed and highly valuable comments.

This work was financially supported by the Russian Foundation for Basic Research (Project No. 15-01-05369).

Conflicts of Interest: The author declares no conflict of interest.

\section{References}

1. Markov, A.A. On free topological groups. Dokl. Akad. Nauk SSSR 1941, 31, 299-301.

2. Markov, A.A. On free topological groups. Am. Math. Soc. Transl. 1950, 30, 11-88.

3. Graev, M.I. Free topological groups. Am. Math. Soc. Transl. 1962, 8, 305-364.

4. Graev, M.I. The theory of topological groups I. Uspekhi Mat. Nauk 1950, 5, 3-56.

5. Nakayama, T. Note on free topological groups. Proc. Imp. Acad. Tokyo 1953, 19, 471-475.

6. Kakutani, S. Free topological groups and infinite direct product of topological groups. Proc. Imp. Acad. Tokyo 1944, 20, 595-598.

7. Mal'tsev, A.I. Free topological algebras. Am. Math. Soc. Transl. Ser. II 1961, 17, 173-200.

8. Morris, S.A. Varieties of topological groups. Bull. Aust. Math. Soc. 1969, 1, 145-160.

9. Morris, S.A. Varieties of topological groups II. Bull. Aust. Math. Soc. 1970, 2, 1-13.

10. Morris, S.A. Varieties of topological groups III. Bull. Aust. Math. Soc. 1970, 2, 165-178.

11. Morris, S.A. Varieties of topological groups: A survey. Colloq. Math. 1982, 46, 147-165.

12. Higman, G. Unrestricted free products, and varieties of topological groups. J. London Math. Soc. 1952, 27, 73-81.

13. Porst, H.E. On the existence and structure of free topological groups. In Category Theory at Work; Herrlich, H., Porst, H.E., Eds.; Heldermann: Berlin, Germany, 1991; pp. 165-176.

14. Comfort, W.W.; van Mill, J. On the existence of free topological groups. Topol. Appl. 1988, 29, 245-265.

15. Kopperman, R.D.; Mislove, M.W.; Morris, S.A.; Nickolas, P.; Pestov, V.; Svetlichny, S. Limit laws for wide varieties of topological groups. Houston J. Math. 1996, 22, 307-328.

16. Dikranjan, D.; Tkachenko, M. Varieties generated by countably compact Abelian groups. Proc. Am. Math. Soc. 2002, 130, 2487-2496.

17. Arhangel'skii, A.; Tkachenko, M. Topological Groups and Related Structures; Atlantis/World Sci.: Amsterdam, The Netherlands, 2008.

18. Hart K.P.; van Mill, J. A separable normal topological group which is not Lindelöf. Topol. Appl. 1985, 20, 279-287.

19. Genze, L.V. Free Boolean topological groups. Vestn. Tomsk. Gos. Univ. 2006, 290, 11-13.

20. Genze, L.V.; Gul'ko, S.P.; Khmyleva, T.E. Classification of the free Boolean topological groups on ordinals. Vestn. Tomsk. Gos. Univ. Mat. Mekh. 2008, 1, 23-31.

21. Genze, L.V.; Gul'ko, S.P.; Khmyleva, T.E. Classification of continuous n-valued function spaces and free periodic topological groups for ordinals. Topol. Proc. 2011, 38, 1-15.

22. Sipacheva, O.V. The topology of free topological groups. J. Math. Sci. 2005, 131, 5765-5838.

23. Tkačenko, M.G. On the topology of Markov free groups. In $V$ Symposium on General Topology; Institute of Mathematics of the Czechoslovak Academy of Sciences: Prague, Czechoslovakia, 1982. 
24. Tkačenko, M.G. On topologies of free groups. Czechoslovak Math. J. 1984, 34, 541-551.

25. Pestov, V.G. Neighborhoods of identity in free topological groups. Vestn. Mosk. Univ. Ser. 1. Mat. Mekh. 1985, 3, 8-10.

26. Sipacheva, O.V. A description of the free group topology without application of universal uniform structures. In General Topology. Mappings of Topological Spaces; Izd. Mosk. Univ.: Moscow, Russia, 1986; pp. 122-130. (In Russian)

27. Sipacheva, O.V. Free topological groups of spaces and their subspaces. Topol. Appl. 2000, 101, 181-212.

28. Tkachenko, M.G. On completeness of free Abelian topological groups. Soviet Math. Dokl. 1983, 27, 341-345.

29. Uspenskii, V.V. Free topological groups of metrizable spaces. Math. USSR-Izv. 1991, 37, 657-680.

30. Przymusiński, T. Collectionwise normality and extensions of continuous functions. Fundam. Math. 1978, 98, 75-81.

31. Arkhangel'skii, A.V. Topological Spaces and Continuous Mappings. Remarks on Topological Groups; Moskov. Univ.: Moscow, Russia, 1969. (In Russian)

32. Arnautov, V.I.; Arkhangel'skii, A.V.; Kirku, P.I.; Mikhalev, A.V.; Mukhin, Yu.N.; Protasov, I.V.; Choban, M.M. Unsolved Problems in Topological Algebra; Preprint; Shtiintsa: Kishinev, USSR, 1985. (In Russian)

33. Mack, J.; Morris, S.A.; Ordman, E.T. Free topological groups and the projective dimension of locally compact Abelian groups. Proc. Am. Math. Soc. 1973, 40, 303-308.

34. Tkachenko, M.G. Strict collection-wise normality and countable compactness in free topological groups. Sibirsk. Mat. Zh. 1987, 28, 167-177.

35. Sipacheva, O.V. On free topological groups with the inductive limit topologies. Ann. N.Y. Acad. Sci. 1996, 788, 188-196.

36. Tkachuk, V.V. Duality with respect to the functor $C_{p}$ and cardinal invariants of the type of the Souslin number. Math. Notes 1985, 37, 247-252.

37. Engelking, R. General topology; Heldermann: Berlin, Germany, 1989.

38. De Groot, J. Non-Archimedean metrics in topology. Proc. Am. Math. Soc. 1956, 7, 948-953.

39. Pestov, V.G. Compactly generated topological groups. Math. Notes 1986, 40, 880-882.

40. Tkachenko, M.G. Countably compact and pseudocompact topologies on free Abelian groups. Sov. Math. 1990, 34, 79-86.

41. Hewitt, E.; Ross, K.A. Abstract Harmonic Analysis; Springer: Berlin, Germany, 1963; Volume 1.

42. Hewitt, E.; Ross, K.A. Extensions of Haar measure and of harmonic analysis for locally compact Abelian groups. Math. Ann. 1965, 160, 171-194.

43. Arkhangel'skii, A.V. Mappings related to topological groups. Soviet Math. Dokl. 1968, 9, 1011-1015.

44. Thomas, B.V.S. Free topological groups. Gen. Topol. Appl. 1974, 4, 51-72.

45. Joiner, C. Free topological groups and dimension. Trans. Am. Math. Soc. 1976, 220, 401-418. 
46. Hardy, J.P.L.; Morris, S.A.; Thompson, H.B. Applications of the Stone-Čech compactification to free topological groups. Proc. Am. Math. Soc. 1976, 55, 160-164.

47. Frolík, Z. Fixed points of maps of extremally disconnected spaces and complete Boolean algebras. Bull. Acad. Polon. Sci. Ser. Math. Astron. Phys. 1968, 16, 269-275.

48. Gillman, L.; Jerison, M. Rings of Continuous Functions; Van Nostrand: Princeton, NJ, USA, 1960.

49. Arkhangel'skii, A.V. Relations among the invariants of topological groups and their subspaces. Russ. Math. Surveys 1980, 35, 1-23.

50. Okunev, O.G. A method for constructing examples of $M$-equivalent spaces. Topol. Appl. 1990, 36, 157-171.

51. Tkachuk, V.V. On a method of constructing examples of M-equivalent spaces. Russ. Math. Surveys 1983, 38, 135-136.

52. Arkhangel'skii, A.V. Every extremally disconnected bicompactum of weight $\mathfrak{c}$ is inhomogeneous. Soviet Math. Dokl. 1967, 8, 897-900.

53. Louveau, A. Sur un article de S. Sirota. Bull. Sci. Math. (France) 1972, 96, 3-7.

54. Malyhin, V.I. Extremally disconnected and similar groups. Soviet Math. Dokl. 1975, 16, 21-25.

55. Malykhin, V.I. On extremally disconnected topological groups. Russian Math. Surveys 1979, 34, 67-76.

56. Sirota, S. The product of topological groups and extremal disconnectedness. Math. USSR-Sb. 1969, 8, 169-180.

57. Zelenyuk, E.G. Topological groups with finite semigroups of ultrafilters. Mat. Studii 1996, $6,41-52$.

58. Zelenyuk, E.G. Extremal ultrafilters and topologies on groups. Mat. Studii 2000, 14, 121-140.

59. Zelenyuk, Y.G. Ultrafilters and Topologies on Groups; De Gruyter: Berlin, Germany, 2011.

60. Sipacheva, O.V. The nonexistence of extremally disconnected free topological groups. Topol. Appl. 2013, 160, 1227-1231.

61. Sipacheva, O.V. Nonexistence of countable extremally disconnected groups with many open subgroups. Topol. Appl. 2015, 179, 193-199.

62. Arhangel'skii, A. A study of extremally disconnected topological spaces. Bull. Math. Sci. 2011, 1, 3-12.

63. Enayat, A. Analogues of the MacDowell-Specker theorem for set theory. In Models, Algebras, and Proofs; Caicedo, X., Montenegro, C.H., Eds.; CRC Press: Boca Raton, FL, USA, 1996; pp. 25-50.

64. Jech, T. Set Theory; Springer: Berlin, Germany, 2003.

65. Brendle, J. Strolling through paradise. Fund. Math. 1995, 148, 1-25.

66. Thümmel, E. Private communication.

67. Judah, H.; Shelah, S. $\Delta_{2}^{1}$-sets of reals. Ann. Pure Appl. Logic 1989, 42, 207-223.

68. Nyikos, P.J. Subsets of ${ }^{\omega} \omega$ and the Fréchet-Urysohn and $\alpha_{i}$-properties. Topol. Appl. 1992, $48,91-116$.

69. Hrušák, M.; Ramos-García, U.A. Malykhin's problem. Adv. Math. 2014, 262, 193-212. 
70. Sipacheva, O.V. A class of free locally convex spaces. Sb. Math. 2003, 194, 333-360.

71. Arkhangel'skii, A.V. Algebraic objects generated by topological structure. J. Sov. Math. 1989, 45, 956-990.

72. Fay, T.; Ordman, E.; Thomas, B.V.S. The free topological group over rationals. Gen.T̃opol.Ãppl. 1979, 10, 33-47.

73. Reznichenko, E.A.; Sipacheva, O.V. Quotient mappings to words of bounded length in free topological groups. In General Topology. Mappings, Products, and Dimension of Spaces; Izd. Mosk. Univ.: Moscow, Russia, 1995; pp. 98-119. (In Russian) 


\title{
Lindelöf $\Sigma$-Spaces and $\mathbb{R}$-Factorizable Paratopological Groups
}

\author{
Mikhail Tkachenko
}

Abstract: We prove that if a paratopological group $G$ is a continuous image of an arbitrary product of regular Lindelöf $\Sigma$-spaces, then it is $\mathbb{R}$-factorizable and has countable cellularity. If in addition, $G$ is regular, then it is totally $\omega$-narrow and satisfies $\operatorname{cel}_{\omega}(G) \leq \omega$, and the Hewitt-Nachbin completion of $G$ is again an $\mathbb{R}$-factorizable paratopological group.

Reprinted from Axioms. Cite as: Tkachenko, M. Lindelöf $\Sigma$-Spaces and $\mathbb{R}$-Factorizable Paratopological Groups. Axioms 2016, 4, 254-267.

\section{Introduction}

Our main objective is the study of paratopological groups that can be represented as continuous images of products of Lindelöf $\Sigma$-spaces. While the properties of (para)topological groups that are Lindelöf $\Sigma$-spaces (referred to as Lindelöf $\Sigma$-groups) are well-understood [1-4], our knowledge about the former class of groups is very modest. The lack of the continuity of the inverse in paratopological groups makes our job more difficult when compared to the case of topological groups. In fact, most of our technique is essentially asymmetric.

Topological groups representable as continuous images of products of Lindelöf $\Sigma$-spaces were studied in [5], where it was shown that every uncountable regular cardinal was a weak precaliber for any group $G$ in this class and that $G$ satisfied $\operatorname{cel}_{\omega}(G) \leq \omega$. According to [2] (Corollary 3.5), a slightly weaker result is valid for Tychonoff paratopological groups representable as continuous images of products of Lindelöf $\Sigma$-spaces: these groups $G$ satisfy the inequality $\operatorname{cel}_{\omega}(G) \leq \omega$. However, the justification of this fact given in [2] contains a gap. In a few words, the problem with the argument in [2] is the existence of a weak $\sigma$-lattice of open continuous mappings of a given completely regular paratopological group $G$ onto Hausdorff spaces with a $G_{\delta}$-diagonal (see Definition 6). As far as we know, all other results in [2] are proven correctly. It is a simple exercise to show that every Hausdorff topological group has the required lattice of open mappings, while the case of paratopological groups is much more elusive.

It follows from our lemmas 9 and 11 that every weakly Lindelöf regular paratopological group has a weak $\sigma$-lattice of continuous open mappings onto Hausdorff spaces with a $G_{\delta}$-diagonal. Since every space representable as a 
continuous image of a product of Lindelöf $\Sigma$-spaces is weakly Lindelöf, these facts fill in the gap in the proof of [2] (Corollary 3.5) (see our Theorem 13).

It turns out that the paratopological groups $G$, which are continuous images of products of Lindelöf $\Sigma$-spaces, have several properties that make them look like Lindelöf $\Sigma$-groups. For example, we prove in Theorem 12 that such a group $G$ is $\mathbb{R}$-factorizable and has countable cellularity. If in addition the group $G$ is regular, then it is totally $\omega$-narrow and satisfies $\operatorname{cel}_{\omega}(G) \leq \omega$, and the Hewitt-Nachbin completion of $G$ is again an $\mathbb{R}$-factorizable paratopological group containing $G$ as a dense subgroup (see Theorem 13). This fact is one of the first results on the preservation of the paratopological group structure under taking the Hewitt-Nachbin completion: almost all known results of this kind refer to topological groups, and their proofs depend essentially on the continuity of the inverse.

Finally, in Section 4, we formulate several open problems regarding paratopological groups representable as continuous images of products of Lindelöf $\Sigma$-spaces. We are mainly interested in finding out whether the conclusions " $G$ is totally $\omega$-narrow and satisfies $\mathrm{cel}_{\omega}(G) \leq \omega^{\prime \prime}$ in Theorem 13 can be extended to Hausdorff paratopological groups $G$.

The article is organized as follows. In Section 2, we introduce a class $\mathcal{L} \Sigma$ of Hausdorff spaces that contains the Lindelöf $\Sigma$-spaces and shares many properties with the latter one. The advantage of working with spaces from the class $\mathcal{L} \Sigma$ resides in the fact that this class is stable with respect to taking Hausdorff continuous images. We collect several results about the permanence properties of the class $\mathcal{L} \Sigma$ and present more facts that will be used in Section 3.

Section 3 contains our main results about paratopological groups representable as continuous images of products of Lindelöf $\Sigma$-spaces. A few selected problems related to the material of Section 3 are given with comments in Section 4.

\section{Preliminaries}

A space $X$ is weakly Lindelöf if every open cover of $X$ contains a countable subfamily whose union is dense in $X$. Every space with a dense Lindelöf subspace or having countable cellularity is weakly Lindelöf.

According to [6], a Hausdorff space $X$ is called a Lindelöf $\Sigma$-space if there exist a countable family $\mathcal{F}$ of closed sets in $X$ and a cover $\mathcal{C}$ of $X$ by compact sets, such that for every $C \in \mathcal{C}$ and every open neighborhood $U$ of $C$ in $X$, one can find $F \in \mathcal{F}$, such that $C \subseteq F \subseteq U$. In fact, K. Nagami defined in [6] the wider class of $\Sigma$-spaces, so the Lindelöf $\Sigma$-spaces are simply the $\Sigma$-spaces with the Lindelöf property. The reader can find a detailed discussion of distinct ways to define Lindelöf $\Sigma$-spaces in [7] (Theorem 1).

It is known that the class of Lindelöf $\Sigma$-spaces is countably productive and that an $F_{\sigma}$-subset of a Lindelöf $\Sigma$-space is again a Lindelöf $\Sigma$-space [6]. This class of spaces 
becomes especially stable when one restricts himself to considering Tychonoff spaces only. It turns out that every continuous image, say $Y$ of a Lindelöf $\Sigma$-space $X$, is again a Lindelöf $\Sigma$-space, provided that $X$ and $Y$ are Tychonoff [1], (Proposition 5.3.5). In fact, the same conclusion remains valid if $X$ is Hausdorff and $Y$ is regular [4] (Lemma 4.5). However, we do not know whether the latter fact can be extended to the case when both $X$ and $Y$ are Hausdorff. This is why we define here a (possibly) wider class $\mathcal{L} \Sigma$ of Hausdorff spaces that is countably productive and is closed under taking continuous images.

Definition 1. A Hausdorff space $X$ is in the class $\mathcal{L} \Sigma$ if there exist a countable family $\mathcal{F}$ of (not necessarily closed) subsets of $X$ and a cover $\mathcal{C}$ of $X$ by compact subsets, such that for every $C \in \mathcal{C}$ and every open neighborhood $U$ of $C$ in $X$, one can find $F \in \mathcal{F}$, such that $C \subseteq F \subseteq U$.

It follows from Definition 1 that every Lindelöf $\Sigma$-space is in the class $\mathcal{L} \Sigma$. It is also easy to verify that every space $X \in \mathcal{L} \Sigma$ is Lindelöf. Therefore, a regular space in $\mathcal{L} \Sigma$ is normal (hence, Tychonoff), so regular spaces in $\mathcal{L} \Sigma$ are Lindelöf $\Sigma$-spaces according to [7] (Theorem 1).

Proposition 2. The class $\mathcal{L} \Sigma$ is countably productive and closed under taking continuous images. Further, if $Y$ is an $F_{\sigma}$-subset of a space $X \in \mathcal{L} \Sigma$, then $Y \in \mathcal{L} \Sigma$.

Proof. Let $\left\{X_{k}: k \in \omega\right\} \subseteq \mathcal{L} \Sigma$ be a family of spaces. For every $k \in \omega$, let $\mathcal{F}_{k}$ and $\mathcal{C}_{k}$ be families of subsets of $X_{k}$ witnessing that $X_{k} \in \mathcal{L} \Sigma$. We can assume that $X_{k} \in \mathcal{F}_{k}$ for each $k \in \omega$. To show that $X=\prod_{k \in \omega} X_{k}$ is in $\mathcal{L} \Sigma$, we define families $\mathcal{F}$ and $\mathcal{C}$ of subsets of $X$ as follows.

Let $\mathcal{F}$ be the family of sets of the form $\prod_{k \in \omega} F_{k}$, where $F_{k} \in \mathcal{F}_{k}$ for each $k \in \omega$ and $F_{k} \neq X_{k}$ for at most finitely many indices $k \in \omega$. Clearly the family $\mathcal{F}$ is countable. Similarly, let $\mathcal{C}$ be the family of sets of the form $\prod_{k \in \omega} C_{k}$, where $C_{k} \in \mathcal{C}_{k}$ for each $k \in \omega$. Then, the family $\mathcal{C}$ consists of compact subsets of $X$. Take an element $C \in \mathcal{C}$ and an open neighborhood $U$ of $C$ in $X$. Then, $C=\prod_{k \in \omega} C_{k}$, where $C_{k} \in \mathcal{C}_{k}$ for each $k \in \omega$. By Wallace's Lemma, there exists a finite set $A \subseteq \omega$ and open sets $O_{k} \subseteq X_{k}$ with $k \in A$, such that $C \subseteq \prod_{k \in \omega} V_{k} \subseteq U$, where $V_{k}=O_{k}$ if $k \in A$ and $V_{k}=X_{k}$ if $k \in \omega \backslash A$. For every $k \in A$, there exists $F_{k} \in \mathcal{F}_{k}$, such that $C_{k} \subseteq F_{k} \subseteq O_{k}$. Let $F=\prod_{k \in \omega} E_{k}$, where $E_{k}=F_{k}$ if $k \in A$ and $E_{k}=X_{k}$ if $k \in \omega \backslash A$. Then, $F \in \mathcal{F}$ and $C \subseteq F \subseteq \prod_{k \in \omega} V_{k} \subseteq U$. Therefore, the families $\mathcal{F}$ and $\mathcal{C}$ witness that $X \in \mathcal{L} \Sigma$. This proves that the class $\mathcal{L} \Sigma$ is countably productive.

Let $f: X \rightarrow Y$ be a continuous onto mapping of Hausdorff spaces, where $X \in \mathcal{L} \Sigma$. Take families $\mathcal{F}$ and $\mathcal{C}$ of subsets of $X$ witnessing that $X \in \mathcal{L} \Sigma$. It is easy to verify that the families $\mathcal{F}_{Y}=\{f(F): F \in \mathcal{F}\}$ and $\mathcal{C}_{Y}=\{f(C): C \in \mathcal{C}\}$ of subsets of $Y$ witness that $Y \in \mathcal{L} \Sigma$. 
Finally, let $Y=\bigcup_{k \in \omega} B_{k}$, where each $B_{k}$ is a closed subset of a space $X \in \mathcal{L} \Sigma$. Denote by $\mathcal{F}$ and $\mathcal{C}$ families of subsets of $X$ witnessing that $X \in \mathcal{L} \Sigma$, where $\mathcal{F}$ is countable and each $C \in \mathcal{C}$ is compact. Let us verify that the families:

$$
\mathcal{F}_{Y}=\left\{F \cap B_{k}: F \in \mathcal{F}, k \in \omega\right\}
$$

and:

$$
\mathcal{C}_{Y}=\left\{C \cap B_{k}: C \in \mathcal{C}, k \in \omega\right\}
$$

witness that $Y \in \mathcal{L} \Sigma$. It is clear that $\left|\mathcal{F}_{Y}\right| \leq \omega$ and that each element of $\mathcal{C}_{Y}$ is a compact subset of $Y$. Let $K=C \cap B_{k}$ be an element of $\mathcal{C}_{Y}$, where $C \in \mathcal{C}$ and $k \in \omega$. Let also $V$ be an open neighborhood of $K$ in $Y$. Then, there exists an open set $O$ in $X$, such that $O \cap Y=V$. Since the compact set $C^{\prime}=C \backslash O$ is disjoint from $K$ and the space $X$ is Hausdorff, we can find disjoint open in $X$ neighborhoods $W_{1}$ and $W_{2}$ of $K$ and $C^{\prime}$, respectively. The set $W^{*}=W_{2} \backslash B_{k}$ is open in $X$ and contains $C^{\prime}$. Hence, the set $U=O \cup W^{*}$ is an open neighborhood of $C$ in $X$, so we can find an element $F \in \mathcal{F}$, such that $C \subseteq F \subseteq U$. Then, $F \cap B_{k}$ is an element of $\mathcal{F}_{Y}$ that satisfies:

$$
K \subseteq F \cap B_{k} \subseteq U \cap B_{k}=O \cap B_{k} \subseteq O \cap Y=V
$$

This completes the proof of the fact that $Y \in \mathcal{L} \Sigma$.

Another important property of the spaces in $\mathcal{L} \Sigma$ is presented in the following result, which is close to [1] (Proposition 5.3.15). However, our proof of Proposition 3 is quite different from the one given in [1], since we work in the class of Hausdorff spaces, which is much wider than the class of Tychonoff spaces considered in [1] (Section 5.3).

Proposition 3. If a space $X \in \mathcal{L} \Sigma$ admits a continuous one-to-one mapping onto a Hausdorff space $Y$ with a countable network, then $X$ itself has a countable network.

Proof. Let $f: X \rightarrow Y$ be a continuous bijection. It is well known that every Hausdorff space with a countable network admits a continuous one-to-one mapping onto a second countable Hausdorff space. Let $i: Y \rightarrow Z$ be a continuous bijection of $Y$ onto a second countable Hausdorff space $Z$. Then, $g=i \circ f$ is a continuous bijection of $X$ onto $Z$. Denote by $\mathcal{B}$ a countable base for $Z$. We can assume that $\mathcal{B}$ is closed under finite intersections and finite unions.

Let families $\mathcal{F}$ and $\mathcal{C}$ of subsets of $X$ witness that $X \in \mathcal{L} \Sigma$, where $|\mathcal{F}| \leq \omega$ and each $C \in \mathcal{C}$ is compact. We claim that the countable family:

$$
\mathcal{N}=\left\{F \cap g^{-1}(W): F \in \mathcal{F}, W \in \mathcal{B}\right\}
$$


is a network for $X$. Indeed, take a point $x \in X$ and an open neighborhood $U$ of $x$ in $X$. There exists $C \in \mathcal{C}$, such that $x \in C$. Then, $K=C \backslash U$ is a compact subset of $X$ and $x \notin K$. Hence, the compact subset $g(K)$ of $Z$ does not contain the point $g(x)$, and we can find disjoint elements $W, W^{\prime} \in \mathcal{B}$, such that $g(x) \in W$ and $g(K) \subseteq W^{\prime}$. Then, $O=U \cup g^{-1}\left(W^{\prime}\right)$ is an open neighborhood of $C$ in $X$, so there exists an element $F \in \mathcal{F}$, such that $C \subseteq F \subseteq O$. It is clear that $F \cap g^{-1}(W)$ is an element of $\mathcal{N}$, and we have that:

$$
x \in F \cap g^{-1}(W) \subseteq O \cap g^{-1}(W)=U \cap g^{-1}(W) \subseteq U
$$

We have thus proven that $\mathcal{N}$ is a countable network for $X$.

Replacing the family $\mathcal{N}$ in the proof of Proposition 3 with the family:

$$
\mathcal{N}^{\prime}=\left\{F \cap \overline{g^{-1}(W)}: F \in \mathcal{F}, W \in \mathcal{B}\right\}
$$

we obtain the following version of the proposition:

Proposition 4. If a Lindelöf $\Sigma$-space $X$ admits a continuous one-to-one mapping onto a Hausdorff space with a countable network, then X has a countable network of closed sets.

The following lemma was proven in [2] for regular Lindelöf $\Sigma$-spaces. Therefore, we extend the corresponding result from [2] to the wider class of Hausdorff $\mathcal{L} \Sigma$-spaces.

Lemma 5. If a space $X \in \mathcal{L} \Sigma$ has a $G_{\delta}$-diagonal, then it has a countable network.

Proof. Suppose that $X \in \mathcal{L} \Sigma$. Then, Proposition 2 implies that $X^{2} \in \mathcal{L} \Sigma$, so the space $X^{2}$ is Lindelöf. Let $\left\{U_{n}: n \in \omega\right\}$ be a family of open neighborhoods of the diagonal $\Delta_{X}$ in $X^{2}$ such that $\Delta_{X}=\bigcap_{n \in \omega} U_{n}$. It is clear that $F_{n}=X^{2} \backslash U_{n}$ is a closed Lindelöf subspace of $X^{2}$. Given $n \in \omega$ and a point $(x, y) \in F_{n}$, we can find disjoint open neighborhoods $V_{n}(x, y)$ and $W_{n}(x, y)$ of the points $x$ and $y$, respectively, in $X$. The open cover $\left\{V_{n}(x, y) \times W_{n}(x, y):(x, y) \in F_{n}\right\}$ of the Lindelöf space $F_{n}$ contains a countable subcover, say $\left\{V_{n}(x, y) \times W_{n}(x, y):(x, y) \in C_{n}\right\}$, where $C_{n}$ is a countable subset of $F_{n}$. Let:

$$
\gamma=\left\{V_{n}(x, y): n \in \omega,(x, y) \in C_{n}\right\} \cup\left\{W_{n}(x, y): n \in \omega,(x, y) \in C_{n}\right\}
$$

Then, $\gamma$ is a countable family of open sets in $X$. We claim that for every pair $a, b$ of distinct points in $X$, there exist disjoint elements $V, W \in \gamma$, such that $a \in V$ and $b \in W$. Indeed, since $(a, b) \in X^{2} \backslash \Delta_{X}$, there exists $n \in \omega$, such that $(a, b) \notin U_{n}$, i.e., $(a, b) \in F_{n}$. Hence, there exists an element $(x, y) \in C_{n}$, such that $(a, b) \in$ $V_{n}(x, y) \times W_{n}(x, y)$. This means that $V=V_{n}(x, y) \in \gamma$ and $W=W_{n}(x, y) \in \gamma$ are 
disjoint open neighborhoods of the points $a$ and $b$, respectively. This proves our claim.

Let $\mathcal{B}$ be the family of finite intersections of elements of $\gamma$. It is clear that $\mathcal{B}$ is a base for a Hausdorff topology $\tau$ on $X$. Then, the space $Y=(X, \tau)$ has a countable base, and the identity mapping of $X$ onto $Y$ is a continuous bijection. Applying Proposition 3, we conclude that $X$ has a countable network.

Given continuous mappings $g: X \rightarrow Y$ and $h: X \rightarrow Z$, we will write $g \prec h$ if there exists a continuous mapping $p: Y \rightarrow Z$ satisfying $h=p \circ g$.

We will also need the notion of a weak $\sigma$-lattice of mappings mentioned in the Introduction (see also [2], Definition 3.1).

Definition 6. Let $Y$ be a space and $\mathcal{L}$ a family of continuous mappings of $Y$ elsewhere. Then, $\mathcal{L}$ is said to be a weak $\sigma$-lattice for $Y$ if the following conditions hold:

(1) $\mathcal{L}$ generates the original topology of $Y$;

(2) every finite subfamily of $\mathcal{L}$ has a lower bound in $(\mathcal{L}, \prec)$;

(3) for every decreasing sequence $p_{0} \succ p_{1} \succ p_{2} \succ \cdots$ in $\mathcal{L}$, there exists $p \in \mathcal{L}$ and $a$ continuous one-to-one mapping $\phi: p(Y) \rightarrow q(Y)$, such that $q=\phi \circ p$, where $q$ is the diagonal product of the family $\left\{p_{n}: n \in \omega\right\}$.

A typical example of a weak $\sigma$-lattice for a topological group $H$ is the family of all quotient mappings $\pi_{N}: H \rightarrow H / N$ onto left coset spaces, where $N$ is an arbitrary closed subgroup of type $G_{\delta}$ in $H$.

Let us recall that a $G_{\delta, \Sigma}$-set in a space $X$ is the union of an arbitrary family of $G_{\delta}$-sets in $X$. Further, a space $Y$ is said to be $\omega$-cellular or, in symbols, cel $\omega(Y) \leq \omega$ if every family $\gamma$ of $G_{\delta}$-sets in $Y$ contains a countable subfamily $\lambda$, such that $\bigcup \lambda$ is dense in $\bigcup \gamma$. It is clear that every $\omega$-cellular space has countable cellularity. In fact, the class of $\omega$-cellular spaces is considerably narrower than the class of spaces of countable cellularity. For example, a space $Y$ of countable pseudo-character satisfies $\operatorname{cel}_{\omega}(Y) \leq \omega$ if and only if it is hereditarily separable.

Our next result is a special case of [2] (Theorem 3.4), which is sufficient for our purposes. We supply it with a short proof based on another fact from [2].

Theorem 7. Let $X=\prod_{i \in I} X_{i}$ be a product of regular Lindelöf $\Sigma$-spaces and a Tychonoff space $Y$ be a continuous image of $X$. If $Y$ has a weak $\sigma$-lattice of open mappings onto Hausdorff spaces with a $G_{\delta}$-diagonal, then $\operatorname{cel}_{\omega}(Y) \leq \omega$, and the closure of every $G_{\delta, \Sigma}$-subset of $Y$ is $a G_{\delta}$-set.

Proof. First, we choose a point $a \in X$. For every countable set $J \subseteq I$, denote by $p_{J}$ the projection of $X$ onto the sub-product $X_{J}=\prod_{i \in J} X_{i}$. Then, $X_{J}$ is a Lindelöf $\Sigma$-space, 
and we identify it with a corresponding closed subspace of $X$ multiplying $X_{J}$ by the singleton $\left\{p_{J}(a)\right\}$. Then, the family:

$$
\left\{p_{J}: J \subseteq I,|J| \leq \omega\right\}
$$

constitutes a strong $\sigma$-lattice of open retractions of $X$ onto Lindelöf $\Sigma$-subspaces (see [2], Definition 3.1).

Let $f: X \rightarrow Y$ be a continuous onto mapping. Denote by $\mathcal{L}$ a weak $\sigma$-lattice of open mappings of $Y$ onto Hausdorff spaces with a $G_{\delta}$-diagonal. For every $\varphi \in \mathcal{L}$, the composition $g=\varphi \circ f$ is a continuous mapping of $X$ onto the Hausdorff space $\varphi(Y)$ with a $G_{\delta}$-diagonal. By [8] (Theorem 1 ), $g$ depends at most on countably many coordinates, so we can find a countable set $J \subseteq I$ and a mapping $h_{J}: X_{J} \rightarrow \varphi(Y)$, such that $g=h_{J} \circ p_{J}$. Since $p_{J}$ is an open continuous mapping, $h_{J}$ is continuous. Hence, $\varphi(Y)$ is in the class $\mathcal{L} \Sigma$ as a continuous image of the Lindelöf $\Sigma$-space $X_{J}$. By Lemma 5, $\varphi(Y)$ has a countable network for each $\varphi \in \mathcal{L}$. It follows that $X, f, Y$ satisfy the conditions of Theorem 3.3 in [2]; hence, $\operatorname{cel}_{\omega}(Y) \leq \omega$, and the closure of every $G_{\delta, \Sigma}$-subset of $Y$ is a $G_{\delta}$-set in $Y$.

We recall that a paratopological group $G$ is called $\mathbb{R}$-factorizable if for every continuous real-valued function $f$ on $G$, one can find a continuous homomorphism $p: G \rightarrow H$ onto a second countable paratopological group $H$ and a continuous real-valued function $h$ on $H$ satisfying $f=h \circ p$. The original definition of $\mathbb{R}$-factorizable paratopological groups in [9] involves separation restrictions on the groups $G$ and $H$, thus giving rise to the concepts of $\mathbb{R}_{i}$-factorizability for $i=1,2,3$. However, it is shown in [4] that all of these concepts coincide and are equivalent to the one given above.

The following fact is a special case of [10] (Theorem 2.2) formulated in a form convenient for applications in Section 3. More precisely, it will be used in the proof of Theorem 12 to deduce the $\mathbb{R}$-factorizability of paratopological groups representable as continuous images of products of Lindelöf $\Sigma$-spaces.

Proposition 8. Let $f: H \rightarrow M$ be a continuous mapping of a Hausdorff weakly Lindelöf paratopological group $H$ to a metrizable space $M$. Then, one can find a closed subgroup $N$ of type $G_{\delta}$ in $H$ and a continuous mapping $h$ of the left coset space $H / N$ to $M$, such that $H / N$ is Hausdorff and the equality $f=h \circ p$ holds, where $p: G \rightarrow G / N$ is the quotient mapping.

\section{Continuous Images of Products of Lindelöf $\Sigma$-Spaces}

In this section we present the proofs of our main results announced in the Introduction. We start with three auxiliary results, Lemmas 9 to 11. 
Let us recall that a space $X$ is Urysohn if for every pair $x, y$ of distinct points in $X$, there exist open neighborhoods $U_{x}$ and $U_{y}$ of $x$ and $y$, respectively, such that $\overline{U_{x}} \cap \overline{U_{y}}=\varnothing$.

Lemma 9. Let $G$ be a weakly Lindelöf regular paratopological group, $\lambda_{0}$ a countable family of open neighborhoods of the identity element $e$ in $G$ and $U_{0} \in \lambda_{0}$. Then, there exists a closed subgroup $N$ of $G$ satisfying the following conditions, where $\pi_{l}: G \rightarrow G / N$ and $\pi_{r}: G \rightarrow G \backslash N$ are quotient mappings of $G$ onto the left and right coset spaces $G / N$ and $G \backslash N$, respectively:

(a) $N \subseteq \cap \lambda_{0}$;

$\left(\mathrm{b}_{l}\right)$ the space $G / N$ is Urysohn and has a $G_{\delta}$-diagonal;

$\left(\mathrm{b}_{r}\right)$ the space $G \backslash N$ is Urysohn and has a $G_{\delta}$-diagonal;

(c) there exist open neighborhoods $O_{l}$ and $O_{r}$ of the elements $\pi_{l}(e)$ and $\pi_{r}(e)$ in $G / N$ and $G \backslash N$, respectively, such that $\pi_{l}^{-1}\left(O_{l}\right) \subseteq U_{0}$ and $\pi_{r}^{-1}\left(O_{r}\right) \subseteq U_{0}$.

Proof. Denote by $\mathcal{N}(e)$ the family of open neighborhoods of $e$ in $G$. Since $G$ is weakly Lindelöf, it follows from [11] (Theorem 10) that the index of regularity of $G$ is countable. Hence the Hausdorff number of $G$ is also countable [12] (Proposition 3.5), i.e., for every $U \in \mathcal{N}(e)$, there exists a countable family $\lambda \subset \mathcal{N}(e)$, such that $\bigcap_{V \in \lambda} V V^{-1} \subset U$.

We introduce a new group multiplication in $G$ by letting $x * y=y \cdot x$, for all $x, y \in G$. Let $G^{*}$ be the paratopological group $(G, *, \tau)$, where $\tau$ is the topology of $G$. In other words, $G$ and $G^{*}$ differ only in multiplication. Hence, $G^{*}$ is also weakly Lindelöf and has a countable Hausdorff number. Therefore, for every $U \in \mathcal{N}(e)$, there exists a countable family $\lambda \subset \mathcal{N}(e)$, such that $\bigcap_{V \in \lambda} V * V^{-1} \subset U$ or, equivalently, $\bigcap_{V \in \lambda} V^{-1} V \subset U$.

Let $\gamma_{0}=\lambda_{0}$. Making use of the inequalities $H s(G) \leq \omega$ and $H s\left(G^{*}\right) \leq \omega$, one can define a sequence $\left\{\gamma_{n}: n \in \omega\right\}$ of countable subfamilies of $\mathcal{N}(e)$ satisfying the following conditions for each $n \in \omega$ :

(i) For every $V \in \gamma_{n}$, there exists $W \in \gamma_{n+1}$, such that $W^{2} \subset V$;

(ii $\left.{ }_{r}\right) \bigcap_{W \in \gamma_{n+1}} W W^{-1} \subset V$, for each $V \in \gamma_{n}$;

(ii $\left.{ }_{l}\right) \bigcap_{W \in \gamma_{n+1}} W^{-1} W \subset V$, for each $V \in \gamma_{n}$.

Then, $\gamma=\bigcup_{n \in \omega} \gamma_{n}$ is a countable subfamily of $\mathcal{N}(e)$. Let us show that $N=\bigcap \gamma$ is as required.

Since $\lambda_{0}=\gamma_{0} \subseteq \gamma$, it follows that $N \subseteq \bigcap \lambda_{0}$. This implies the validity of (a) of the lemma. Condition ( ii $_{r}$ ) implies that $N N^{-1} \subseteq V$ for every $V \in \gamma_{n}$ and every $n \in \omega$, so $N N^{-1} \subseteq N$. Since $N$ contains the identity $e$ of $G$, we see that $N$ is a subgroup of $G$. Let $\pi_{l}: G \rightarrow G / N$ and $\pi_{r}: G \rightarrow G \backslash N$ be the quotient mappings. By (i), there exists $V \in \gamma_{1} \subset \gamma$, such that $V^{2} \subset U_{0}$. Then, $O_{l}=\pi_{l}(V)$ is an open neighborhood 
of $\pi_{l}(e)$ in $G / N$ and $\pi_{l}^{-1}\left(O_{l}\right)=V N \subset V^{2} \subset U_{0}$. Similarly, $O_{r}=\pi_{r}(V)$ is an open neighborhood of $\pi_{r}(e)$ in $G \backslash N$ and $\pi_{r}^{-1}\left(O_{r}\right)=N V \subset V^{2} \subset U_{0}$. Hence, (c) of the lemma is valid, as well.

Our next step is to show that condition $\left(b_{l}\right)$ of the lemma is also fulfilled, i.e., the coset space $G / N$ is Urysohn and, hence, Hausdorff. In particular, the subgroup $N=\pi_{l}^{-1} \pi_{l}(e)$ is closed in $G$. A similar verification of item $\left(b_{r}\right)$ is left to the reader, since it only requires the use of $\left(\mathrm{ii}_{r}\right)$ in place of $\left(\mathrm{ii}_{l}\right)$.

Take an arbitrary element $x \in G$, such that $x \notin N$. Since the space $G / N$ is homogeneous, it suffices to show that the points $\pi_{l}(e)$ and $\pi_{l}(x)$ have disjoint closed neighborhoods in $G / N$. As $x \notin N$, there exists an element $U \in \gamma_{n}$, for some $n \in \omega$, such that $x \notin U$. By (ii $\left.{ }_{l}\right)$, there exists $V \in \gamma_{n+1}$, such that $x \notin V^{-1} V$. Applying (i) twice, we can find $W \in \gamma_{n+3}$, such that $W^{4} \subseteq V$. Then, $W^{-2} W^{4} \subseteq W^{-4} W^{4} \not \supset x$, whence it follows that:

$$
W^{-1} W^{2} \cap W x W^{-2}=\varnothing
$$

Since the mapping $\pi_{l}$ of $G$ onto $G / N$ is open and $N \subseteq W$ (and, therefore, $N=N^{-1} \subseteq W^{-1}$ ), we have the following inclusions:

$$
\pi_{l}^{-1}\left(\overline{\pi_{l}(W)}\right)=\overline{\pi_{l}^{-1}\left(\pi_{l}(W)\right)}=\overline{W N} \subseteq W^{-1} W N \subseteq W^{-1} W^{2}
$$

and:

$$
\pi_{l}^{-1}\left(\overline{\pi_{l}(W x)}\right)=\overline{\pi_{l}^{-1}\left(\pi_{l}(W x)\right)}=\overline{W x N} \subseteq W x N W^{-1} \subseteq W x W^{-2}
$$

Combining Equations (1) to (3), we see that the closed subsets $\overline{\pi_{l}(W)}$ and $\overline{\pi_{l}(W x)}$ of $G / N$ are disjoint. Since $\pi_{l}(W)$ and $\pi_{l}(W x)$ are open neighborhoods of $\pi_{l}(e)$ and $\pi_{l}(x)$, respectively, in $G / N$, the latter space is Urysohn.

Finally we verify that $G / N$ has a $G_{\delta}$-diagonal. For every $U \in \mathcal{N}(e)$, let:

$$
O_{U}=\bigcup\left\{\pi_{l}(x U) \times \pi_{l}(x U): x \in G\right\}
$$

Then, the countable family $\mathcal{F}=\left\{O_{U}: U \in \gamma\right\}$ of open entourages of the diagonal $\Delta$ in $G / N \times G / N$ satisfies $\Delta=\cap \mathcal{F}$. Indeed, take arbitrary elements $a, b \in G$, such that $\pi_{l}(a) \neq \pi_{l}(b)$. Then, $a^{-1} b \notin N$, so we can find an element $U \in \gamma_{n}$, for some $n \in \omega$, such that $a^{-1} b \notin U$. By (ii $\left.{ }_{l}\right)$, there exists $V \in \gamma_{n+1}$, such that $a^{-1} b \notin V^{-1} V$. Now, we apply (i) to take $W \in \gamma_{n+2}$ with $W^{2} \subseteq V$. We claim that $\left(\pi_{l}(a), \pi_{l}(b)\right) \notin O_{W}$. Indeed, otherwise, there exists $x \in G$, such that $\pi_{l}(a) \in \pi_{l}(x W)$ and $\pi_{l}(b) \in \pi_{l}(x W)$. The latter implies that $a \in x W N$ and $b \in x W N$, whence:

$$
a^{-1} b \in N^{-1} W^{-1} x^{-1} x W N \subset W^{-2} W^{2} \subset V^{-1} V
$$


which is a contradiction. Since the family $\mathcal{F}$ is countable, we conclude that the coset space $G / N$ has a $G_{\delta}$-diagonal. A similar argument shows that the right coset space $G \backslash N$ has a $G_{\delta}$-diagonal. This completes the proof.

The next result is almost evident, so we omit its proof.

Lemma 10. The class of spaces with a $G_{\delta}$-diagonal is countably productive.

Lemma 11. Let $G$ be a weakly Lindelöf regular paratopological group and $\mathcal{A}$ the family of closed subgroups $N$ of $G$ that satisfy conditions $\left(b_{l}\right)$ and $\left(b_{r}\right)$ of Lemma 9. Then, $\mathcal{A}$ is closed under countable intersections.

Proof. Let $\left\{N_{k}: k \in \omega\right\} \subseteq \mathcal{A}$ be a sequence of subgroups of $G$. For every $k \in \omega$, denote by $\pi_{k}$ the quotient mapping of $G$ onto the left coset space $G / N_{k}$. Let also $\varphi$ be the diagonal product of the family $\left\{\pi_{k}: k \in \omega\right\}$. Then, $\varphi$ is a continuous mapping of $G$ to the product space $Z=\prod_{k \in \omega} G / N_{k}$. Each of the factors $G / N_{k}$ has a $G_{\delta}$-diagonal, and so does $Z$, by Lemma 10 . Hence the subspace $\varphi(G)$ of $Z$ also has a $G_{\delta}$-diagonal. Similarly, the space $Z$ and its subspace $\varphi(G)$ are Urysohn since the factors $G / N_{k}$ are Urysohn.

Put $N=\bigcap_{k \in \omega} N_{k}$, and let $\pi: G \rightarrow G / N$ be the quotient mapping. For every $k \in \omega$, there exists a mapping $p_{k}: G / N \rightarrow G / N_{k}$, such that $\pi_{k}=p_{k} \circ \pi$. The mapping $p_{k}$ is continuous and open since so are $\pi$ and $\pi_{k}$. The diagonal product of the family $\left\{p_{k}: k \in \omega\right\}$, say $p$, is a continuous mapping of $G / N$ to $Z=\prod_{k \in \omega} G / N_{k}$. It is clear that $p$ satisfies the equality $\varphi=p \circ \pi$. It is also easy to see that the fibers of the mappings $\varphi$ and $\pi$ coincide, i.e., $p$ is a continuous bijection of $G / N$ onto $\varphi(G)$. Indeed, take arbitrary points $x, y \in G$ with $\varphi(x)=\varphi(y)$. We have to show that $\pi(x)=\pi(y)$. It follows from the definition of $\varphi$ that $\pi_{k}(x)=\pi_{k}(y)$, for each $k \in \omega$. Hence, $x^{-1} y \in \bigcap_{k \in \omega} N_{k}=N$ and $\pi(x)=\pi(y)$. Therefore, the equality $\varphi=p \circ \pi$ implies that $p: G / N \rightarrow \varphi(G)$ is a continuous bijection.

Finally, since the space $\varphi(G)$ is Urysohn and has a $G_{\delta}$-diagonal and $p$ is continuous and one-to-one, we infer that the space $G / N$ is also Urysohn and has a $G_{\delta}$-diagonal. A similar argument shows that the right coset space $G \backslash N$ has the same property. This proves that $N \in \mathcal{A}$.

In the following theorem, we do not impose any separation restriction on the paratopological group $G$.

Theorem 12. Let $X=\prod_{i \in I} X_{i}$ be a product of regular Lindelöf $\Sigma$-spaces and $f: X \rightarrow G$ a continuous mapping of $X$ onto a paratopological group $G$. Then, the group $G$ is $\mathbb{R}$-factorizable and has countable cellularity. 
Proof. Consider a continuous real-valued function $g$ defined on $G$. We can assume the group $G$ is a regular space. Indeed, let $\varphi_{r}: G \rightarrow \operatorname{Reg}(G)$ be the canonical continuous homomorphism, where $\operatorname{Reg}(G)$ is the regularization of $G$ (see $[13,14])$. Then, $\operatorname{Reg}(G)$ is a regular paratopological group, and by the definition of $\operatorname{Reg}(G)$, there exists a continuous real-valued function $g_{r}$ on $\operatorname{Reg}(G)$, such that $g=g_{r} \circ \varphi_{r}$. Hence, $G$ is $\mathbb{R}$-factorizable if so is the group $\operatorname{Reg}(G)$. It also follows from [15] (Proposition 2.2) that the groups $G$ and $\operatorname{Reg}(G)$ have the same cellularity. Notice that $\varphi_{r} \circ f$ is a continuous mapping of $X$ onto $\operatorname{Reg}(G)$. Thus, we can assume that $G$ itself is regular.

By a recent theorem of Banakh and Ravsky in [16], every regular paratopological group is completely regular. Each factor $X_{i}$, being a regular Lindelöf space, is normal and, hence, Tychonoff. Therefore, the product space $X$ is Tychonoff, as well. Our next step is to show that $G$ has a weak $\sigma$-lattice of open mappings onto Hausdorff spaces with a $G_{\delta}$-diagonal.

Take an arbitrary point $x^{*}$ in $X$ and denote by $\sigma\left(x^{*}\right)$ the subspace of $X$ consisting of the points $x \in X$ that differ from $x^{*}$ at most on finitely many coordinates. Clearly $\sigma\left(x^{*}\right)$ is dense in $X$. Since the class of Lindelöf $\Sigma$-spaces is finitely productive (this follows, e.g., from Proposition 2) [1] (Corollary 1.6.45) implies that the subspace $\sigma\left(x^{*}\right)$ of $X$ is Lindelöf. Hence, $f\left(\sigma\left(x^{*}\right)\right)$ is a dense Lindelöf subspace of $G$, so the space $G$ is weakly Lindelöf. Applying Lemma 9, we see that the topology of the group $G$ is initial with respect to the family $\mathcal{L}$ of quotient mappings of $G$ onto Urysohn left coset spaces with a $G_{\delta}$-diagonal, and the same is valid for the family $\mathcal{R}$ of quotient mappings of $G$ onto Urysohn right coset spaces with a $G_{\delta}$-diagonal. Making use of Lemma 11, one can easily prove that both $\mathcal{L}$ and $\mathcal{R}$ are weak $\sigma$-lattices of continuous open mappings for $G$. A routine verification of this fact is omitted.

Since $G$ is a continuous image of the product space $X$, Theorem 7 implies that $\operatorname{cel}_{\omega}(G) \leq \omega$. As $c(G) \leq \operatorname{cel}_{\omega}(G)$, we conclude that $G$ has countable cellularity. It remains to show that the group $G$ is $\mathbb{R}$-factorizable. This requires several steps.

Following the notation in Lemma 11, we denote by $\mathcal{A}$ the family of all closed subgroups $N$ of $G$, such that the coset spaces $G / N$ and $G \backslash N$ are Urysohn and have a $G_{\delta}$-diagonal.

Claim 1. The coset spaces $G / N$ and $G \backslash N$ have a countable network, for each $N \in \mathcal{A}$.

Let $\pi_{N, l}: G \rightarrow G / N$ be the quotient mapping, where $N \in \mathcal{A}$. Then, $f_{N}=\pi_{N, l} \circ f$ is a continuous mapping of $X$ onto the left coset space $G / N$. Notice that $X_{J}=\prod_{i \in J} X_{i}$ is a Lindelöf $\Sigma$-space for every countable set $J \subset I$; hence, [8] (Theorem 1) implies that $f_{N}$ depends on at most countably many coordinates, i.e., one can find a countable set $J \subset I$ and a continuous mapping $h: X_{J} \rightarrow G / N$, such that $f_{N}=h \circ p_{J}$, where $p_{J}: X \rightarrow X_{J}$ is the projection. It is clear that $h$ is a surjective mapping. Applying Proposition 2, we conclude that $G / N \in \mathcal{L} \Sigma$. Hence, by Lemma 5, 
the space $G / N$ has a countable network. The same argument applied to the quotient mapping $\pi_{N, r}: G \rightarrow G \backslash N$ enables us to deduce that the right coset space $G \backslash N$ also has a countable network. This proves Claim 1.

Claim 2. For every $N \in \mathcal{A}$, there exists $M \in \mathcal{A}$, such that $\pi_{M, r} \prec \pi_{N, l}$, and similarly, for every $L \in \mathcal{A}$, there exists $K \in \mathcal{A}$, such that $\pi_{K, l} \prec \pi_{L, r}$.

By the symmetry argument, it suffices to verify the first part of the claim. Let $N$ be a closed subgroup of $G$, such that the left coset space $G / N$ is Urysohn and has a $G_{\delta}$-diagonal. By Claim 1 , the space $G / N$ has a countable network. Denote by $Z$ the semi-regularization of the space $G / N$ (see [14], p. 204), and let $i_{N}: G / N \rightarrow Z$ be the identity mapping. Since $G / N$ is Hausdorff, it follows from [17] (Proposition 1) that the space $Z$ is regular. It is clear that the mapping $i_{N}$ is continuous, so $Z$ has a countable network as a continuous image of the space $G / N$. In particular, $Z$ is Lindelöf and normal. Since $Z$ has a countable network, we can find a continuous bijection $i_{Z}: Z \rightarrow Z_{0}$ onto a separable metrizable space $Z_{0}$. Then, $p=i_{Z} \circ i_{N} \circ \pi_{N, l}$ is a continuous mapping of $G$ onto $Z_{0}$. By Proposition 8 , there exists a closed subgroup $M$ of type $G_{\delta}$ in $G$ and a continuous mapping $q: G \backslash M \rightarrow Z_{0}$, such that $p=q \circ \pi_{M, r}$, where $\pi_{M, r}$ is the quotient mapping of $G$ onto $G \backslash M$. According to Lemma 9 we can assume without loss of generality that $M \in \mathcal{A}$. Let $q_{0}=i_{N}^{-1} \circ i_{Z}^{-1} \circ q$. The mapping $q_{0}$ of $G \backslash M$ to $G / N$ is well defined, since $i_{N}$ and $i_{Z}$ are bijections. Thus, the following diagram commutes.

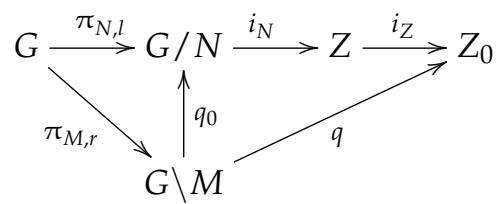

Since $\pi_{N, l}$ and $\pi_{M, r}$ are continuous open mappings, so is $q_{0}$. This implies that $\pi_{M, r} \prec \pi_{N, l}$. Claim 2 is proven.

Claim 3. For every $N \in \mathcal{A}$, there exists $K \in \mathcal{A}$, such that $K \subseteq N$ and $K$ is invariant in $G$.

Indeed, take an arbitrary element $N \in \mathcal{A}$, and let $N_{0}=N$. By Claim 2, there exists $M_{0} \in \mathcal{A}$, such that $\pi_{M_{0}, r} \prec \pi_{N_{0}, l}$. Hence, $M_{0} x \subseteq x N_{0}$ or, equivalently, $M_{0} \subseteq$ $x N_{0} x^{-1}$, for each $x \in G$. Applying Claim 2 once again, we find $N_{1} \in \mathcal{A}$, such that $N_{1} \subseteq x^{-1} M_{0} x$ for each $x \in G$. Continuing this way, we define sequences $\left\{N_{k}: k \in\right.$ $\omega\} \subseteq \mathcal{A}$ and $\left\{M_{k}: k \in \omega\right\} \subseteq \mathcal{A}$, such that $M_{k} \subseteq x N_{k} x^{-1}$ and $N_{k+1} \subseteq x M_{k} x^{-1}$ for each $x \in G$. Then, the subgroup $K=\bigcap_{k \in \omega} M_{k}=\bigcap_{k \in \omega} N_{k}$ of $G$ is as required. Indeed, it follows from Lemma 11 that $K \in \mathcal{A}$, so both coset spaces $G / K$ and $G \backslash K$ are Urysohn and have a $G_{\delta}$-diagonal. It also follows from our definition of $K$ that:

$$
x^{-1} K x \subseteq x^{-1} M_{k} x \subseteq N_{k}
$$


for all $x \in G$ and $k \in \omega$, so $x^{-1} K x \subseteq \bigcap_{k \in \omega} N_{k}=K$. This inclusion is in fact the equality, so $K$ is a closed invariant subgroup of $G$. Since $K \subseteq N_{0}=N$, this completes the proof of Claim 3.

We are now in the position to complete our argument. Let us recall that $g$ is an arbitrary continuous real-valued function on $G$. Since $G$ is Hausdorff and weakly Lindelöf, we apply Proposition 8 to find a closed subgroup $N$ of type $G_{\delta}$ in $G$, such that $g$ is constant on each left coset of $N$ in $G$. Therefore, there exists a real-valued function $h$ on $G / N$, such that $g=h \circ \pi_{N, l}$, where $\pi_{N, l}: G \rightarrow G / N$ is the quotient mapping. Since $\pi_{N, l}$ is continuous and open, the function $h$ is also continuous. By Lemma 9, there exists $N_{1} \in \mathcal{A}$ with $N_{1} \subseteq N$. Then, Claim 3 implies the existence of an invariant subgroup $K$ of $G$, such that $K \in \mathcal{A}$ and $K \subseteq N_{1}$. The inclusions $K \subseteq N_{1} \subseteq N$ mean that there exists a mapping $\pi_{N}^{K}: G / K \rightarrow G_{N}$, such that $\pi_{N, l}=\pi_{N}^{K} \circ \pi_{K}$, where $\pi_{K}: G \rightarrow G / K$ is the quotient homomorphism.

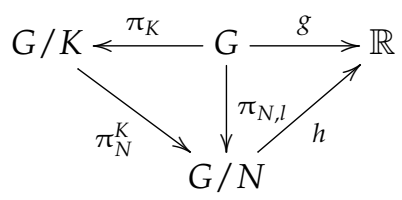

Since the mappings $\pi_{N, l}$ and $\pi_{K}$ are continuous and open, so is $\pi_{N}^{K}$. Hence, $h_{K}=h \circ \pi_{N}^{K}$ is a continuous real-valued function on $G / K$. Notice that $G / K$ is a paratopological group, by the invariance of $K$ in $G$, and $G / K$ is Hausdorff by our choice of $K \in \mathcal{A}$. The group $G / K$ has a countable network by Claim 1; hence, we can apply [9] (Corollary 3.11) according to which $G / K$ is $\mathbb{R}$-factorizable. Therefore, we can find a continuous homomorphism $\varphi: G / K \rightarrow P$ onto a second countable paratopological group $P$ and a continuous real-valued function $h_{P}$ on $P$, such that $h_{K}=h_{P} \circ \varphi$. Therefore, the following diagram commutes.

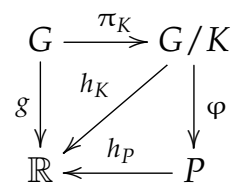

It remains to note that the continuous homomorphism $\psi=\varphi \circ \pi_{K}$ and the function $h_{P}$ satisfy the equality $g=h_{P} \circ \psi$, which implies the $\mathbb{R}$-factorizability of the group $G$.

A topological group $G$ is said to be $\omega$-narrow (see [1], Section 3.4) if it can be covered by countably many translations of any neighborhood of the identity. A paratopological group is totally $\omega$-narrow if it is a continuous homomorphic 
image of an $\omega$-narrow topological group or, equivalently, if the topological group $G^{*}$ associated with $G$ is $\omega$-narrow [12] (Subsection 1.1).

If the paratopological group $G$ in Theorem 12 is regular, we are able to complement the conclusion of the theorem as follows:

Theorem 13. Let $X=\prod_{i \in I} X_{i}$ be a product space, where each $X_{i}$ is a regular Lindelöf $\Sigma$-space and $f: X \rightarrow G$ a continuous mapping of $X$ onto a regular paratopological group $G$. Then, the group $G$ is totally $\omega$-narrow and satisfies cel $\omega(G) \leq \omega$, and the Hewitt-Nachbin completion $v G$ of the group $G$ is again a paratopological group containing $G$ as a dense subgroup. Furthermore, the group $v G$ is $\mathbb{R}$-factorizable.

Proof. Every regular paratopological group is Tychonoff according to [16]. Hence, applying Theorem 12, we conclude that $G$ is a Tychonoff $\mathbb{R}$-factorizable paratopological group. By [17] (Proposition 3.10), $G$ is totally $\omega$-narrow.

The inequality $\operatorname{cel}_{\omega}(G) \leq \omega$ was established in the proof of Theorem 12 under the assumption of the regularity of $G$.

Finally, according to [18] (Theorem 2.3), the Hewitt-Nachbin completion of a Tychonoff $\mathbb{R}$-factorizable paratopological group is again an $\mathbb{R}$-factorizable paratopological group containing the original group as a dense subgroup.

Since the Sorgenfrey line $\mathbb{S}$ is a regular paratopological group that fails to be totally $\omega$-narrow, Theorem 13 implies the following curious fact:

Corollary 14. The Sorgenfrey line $\mathbb{S}$ is not a continuous image of any product of regular Lindelöf $\Sigma$-spaces.

The above corollary also follows from Theorem 12 , since the group $\mathbb{S}$ is not $\mathbb{R}$-factorizable according to [1] (Example 8.1.8). We also note that the conclusion of Corollary 14 is valid for every uncountable subgroup of $\mathbb{S}$.

Remark 1. We present here a direct proof of the fact that the regular group $G$ in Theorem 13 is totally $\omega$-narrow. We hope that it can help to treat the more general case when $G$ is Hausdorff.

Let $\tau$ be the topology of $G$. Denote by $\tau^{-1}$ the family $\left\{U^{-1}: U \in \tau\right\}$. Then, $G^{\prime}=\left(G, \tau^{-1}\right)$ is a paratopological group conjugated to $G$, and the inversion in $G$ is a homeomorphism of $G$ onto $G^{\prime}$. Hence, $G^{\prime}$ is also a continuous image of $X$, so the groups $G$ and $G^{\prime}$ have the same properties. Let $\Delta=\{(x, x): x \in G\}$ be the diagonal in the paratopological group $G \times G^{\prime}$. According to [9] (Lemma 2.2), $\Delta$ is a closed subgroup of $G \times G^{\prime}$ topologically isomorphic to the topological group $G^{*}$ associated with $G$. Therefore, it suffices to show that the group $\Delta$ is $\omega$-narrow. Let $O$ be a neighborhood of the identity $e^{*}$ in $\Delta$. There exists an open neighborhood $U$ of the identity $e$ in $G$, such that $\Delta \cap\left(U \times U^{-1}\right) \subseteq O$. By Lemma 9 and Claims 1 
and 3 in the proof of Theorem 12, we can find a closed invariant subgroup $N$ of $G$, such that the quotient group $G / N$ has a countable network and $\pi^{-1}(V) \subseteq U$ for some open neighborhood $V$ of the identity in $G / N$, where $\pi: G \rightarrow G / N$ is the quotient homomorphism. It is clear that $G^{\prime} / N$ is a paratopological group conjugated to $G / N$ and that $G^{\prime} / N$ has a countable network. Let $\pi^{\prime}: G^{\prime} \rightarrow G^{\prime} / N$ be the quotient homomorphism. Then, $\varphi=\pi \times \pi^{\prime}$ is a continuous homomorphism of $G \times G^{\prime}$ onto the paratopological group $G / N \times G^{\prime} / N$ with a countable network. Clearly, the subgroup $\Delta_{N}=\left\{\left(\pi(x), \pi^{\prime}(x)\right): x \in G\right\}$ of $G / N \times G^{\prime} / N$ also has a countable network and, hence, is Lindelöf. In particular, the group $\Delta_{N}$ is $\omega$-narrow. Therefore, we can find a countable subset $D$ of $\Delta_{N}$, such that $D W=\Delta_{N}=W D$, where $W=\Delta_{N} \cap\left(V \times V^{-1}\right)$ (we identify the groups $G / N$ and $G^{\prime} / N$ algebraically). Let $C$ be a countable subset of $\Delta$, such that $\varphi(C)=D$. It easily follows from our choice of the sets $V$ and $W$ that $\Delta \cap \varphi^{-1}(W) \subseteq \Delta \cap\left(U \times U^{-1}\right) \subseteq O$, so we have the equality $C O=\Delta=O C$. This proves that the topological group $\Delta \cong G^{*}$ is $\omega$-narrow.

\section{Open Problems}

A space $Y$ is said to have the Knaster property if every uncountable family $\gamma$ of open sets in $Y$ contains an uncountable subfamily $\lambda$, such that every two elements of $\lambda$ have a non-empty intersection [1] (Section 5.4). It is clear that every space with the Knaster property has countable cellularity; the converse is valid under $M A$ plus the negation of $\mathrm{CH}$ and fails under $\mathrm{CH}$.

Problem 15. Let a (Hausdorff) paratopological group $G$ be a continuous image of a product of a family of Lindelöf $\Sigma$-spaces. Does $G$ have the Knaster property? Is it $\omega$-narrow?

It is worth mentioning that if $G$ itself is a Lindelöf $\Sigma$-space, then it has the Knaster property and is totally $\omega$-narrow, since the topological group $G^{*}$ associated with $G$ is again a Lindelöf $\Sigma$-space (see, e.g., [9], Corollary 2.3, and [1], Theorem 5.4.7).

Problem 16. Let $G$ be as in Problem 15.

(a) Does the topological group $G^{*}$ associated with $G$ satisfies $c\left(G^{*}\right) \leq \omega$ ?

(b) Is the group $G^{*} \mathbb{R}$-factorizable?

(c) Is the group $G^{*} \omega$-narrow?

What if, in addition, the group $G$ in (a), (b) or (c) is Hausdorff or regular?

Let us note that Theorem 13 answers (c) of Problem 16 in the affirmative for a regular paratopological group $G$. Since every $\mathbb{R}$-factorizable topological group is $\omega$-narrow, the affirmative answer to (b) of Problem 16 would imply the same answer to (c) of the problem. 
Let us recall that a space $Y$ is said to be perfectly $\kappa$-normal if the closure of every open set in $Y$ is a $G_{\delta}$-set. Every metrizable space is evidently perfectly $\kappa$-normal; it is much less evident that arbitrary products of metrizable spaces are also perfectly $\kappa$-normal [19] (Theorem 2).

Problem 17. Let a Hausdorff (regular) paratopological group $G$ be a continuous image of a dense subspace of a product of separable metrizable spaces. Is $G$ perfectly $\kappa$-normal or $\mathbb{R}$-factorizable?

Every paratopological group $G$ admits the natural left quasi-uniformity $\mathcal{L}_{G}$ whose base consists of the sets:

$$
U_{V}^{l}=\left\{(x, y) \in G^{2}: x^{-1} y \in V\right\}
$$

where $V$ runs through all open neighborhoods of the identity in G. Since every quasi-uniformity is generated by a family of upper quasi-uniformly continuous quasi-pseudometrics, the following problem arises in an attempt to show that the group $G$ in Theorem 12 is $\omega$-narrow independently of whether it is regular or not.

Problem 18. Does every upper quasi-uniformly continuous quasi-pseudometric on an arbitrary product of Lindelöf $\sum$-spaces depend at most on countably many coordinates?

Conflicts of Interest: The author declares no conflict of interest.

\section{References}

1. Arhangel'skii, A.V.; Tkachenko, M. G. Topological Groups and Related Structures; van Mill, J., Ed.; Atlantis Press: Paris, France, 2008; Volume I.

2. Tkachenko, M. M-mappings and the cellularity of spaces. Topol. Appl. 1994, 57, 183-207.

3. Uspenskij, V.V. A topological group generated by a Lindelöf $\Sigma$-space has the Souslin property. Sov. Math. Dokl. 1982, 26, 166-169.

4. Xie, L.H.; Lin, S.; Tkachenko, M. Factorization properties of paratopological groups. Topol. Appl. 2013, 160, 1902-1917.

5. Tkachenko, M. M-mappings make their images less cellular. Comment. Math. Univ. Carolin. 1994, 35, 553-563.

6. Nagami, K. $\Sigma$-spaces. Fund. Math. 1969, 65, 169-192.

7. Tkachuk, V. Lindelöf $\Sigma$-spaces: an omnipresent class. Rev. RACSAM 2010, 104, 221-244.

8. Engelking, R. On functions defined on Cartesian products. Fund. Math. 1966, 59, 221-231.

9. Sanchis, M.; Tkachenko, M. $\mathbb{R}$-factorizable paratopological groups. Topol. Appl. 2010, $157,800-808$. 
10. Tkachenko, M. The Hewitt-Nachbin completion of paratopological groups. 2015, in press.

11. Sánchez, I. Cardinal invariants of paratopological groups. Topol. Algebra Appl. 2013, 1, 37-45.

12. Tkachenko, M. Embedding paratopological groups into topological products. Topol. Appl. 2009, 156, 1298-1305.

13. Tkachenko, M. Axioms of separation in semitopological groups and related functors. Topol. Appl. 2013, 161, 364-376.

14. Tkachenko, M. Axioms of separation in paratopological groups and reflection functors. Topol. Appl. 2015, 179, 200-214.

15. Tkachenko, M. Applications of the reflection functors in paratopological groups. Topol. Appl. 2015, doi:10.1016/j.topol.2015.05.080.

16. Banakh, T.; Ravsky, A. A characterization of Tychonoff spaces with applications to paratopological groups. arXiv:1410.1504 [math.GN], 3 December 2014.

17. Banakh, T.; Ravsky, A. The regularity of quotient paratopological groups. arXiv:1003.5409 [math.GN], 4 June 2014.

18. Sanchis, M.; Tkachenko, M. Completions of paratopological groups and bounded sets. 2015, in press.

19. Ščepin, E. V. On K-metrizable spaces. Math. USSR Izvestia 1980, 14, 407-440. 


\section{On T-Characterized Subgroups of Compact Abelian Groups}

\section{Saak Gabriyelyan}

Abstract: A sequence $\left\{u_{n}\right\}_{n \in \omega}$ in abstract additively-written Abelian group $G$ is called a $T$-sequence if there is a Hausdorff group topology on $G$ relative to which $\lim _{n} u_{n}=0$. We say that a subgroup $H$ of an infinite compact Abelian group $X$ is $T$-characterized if there is a $T$-sequence $\mathbf{u}=\left\{u_{n}\right\}$ in the dual group of $X$, such that $H=\left\{x \in X:\left(u_{n}, x\right) \rightarrow 1\right\}$. We show that a closed subgroup $H$ of $X$ is $T$-characterized if and only if $H$ is a $G_{\delta}$-subgroup of $X$ and the annihilator of $H$ admits a Hausdorff minimally almost periodic group topology. All closed subgroups of an infinite compact Abelian group $X$ are $T$-characterized if and only if $X$ is metrizable and connected. We prove that every compact Abelian group $X$ of infinite exponent has a $T$-characterized subgroup, which is not an $F_{\sigma}$-subgroup of $X$, that gives a negative answer to Problem 3.3 in Dikranjan and Gabriyelyan (Topol. Appl. 2013, 160, 2427-2442).

Reprinted from Axioms. Cite as: Gabriyelyan, S. On T-Characterized Subgroups of Compact Abelian Groups. Axioms 2016, 4, 194-212.

\section{Introduction}

Notation and preliminaries: Let $X$ be an Abelian topological group. We denote by $\widehat{X}$ the group of all continuous characters on $X$, and $\widehat{X}$ endowed with the compact-open topology is denoted by $X^{\wedge}$. The homomorphism $\alpha_{X}: X \rightarrow X^{\wedge \wedge}$, $x \mapsto(\chi \mapsto(\chi, x))$, is called the canonical homomorphism. Denote by $\mathbf{n}(X)=$ $\cap_{\chi \in \hat{X}} \operatorname{ker}(\chi)=\operatorname{ker}\left(\alpha_{X}\right)$ the von Neumann radical of $X$. The group $X$ is called minimally almost periodic $(\operatorname{Min} A P)$ if $\mathbf{n}(X)=X$, and $X$ is called maximally almost periodic $(M A P)$ if $\mathbf{n}(X)=\{0\}$. Let $H$ be a subgroup of $X$. The annihilator of $H$ we denote by $H^{\perp}$, i.e., $H^{\perp}=\left\{\chi \in X^{\wedge}:(\chi, h)=1\right.$ for every $\left.h \in H\right\}$.

Recall that an Abelian group $G$ is of finite exponent or bounded if there exists a positive integer $n$, such that $n g=0$ for every $g \in G$. The minimal integer $n$ with this property is called the exponent of $G$ and is denoted by $\exp (G)$. When $G$ is not bounded, we write $\exp (G)=\infty$ and say that $G$ is of infinite exponent or unbounded. The direct sum of $\omega$ copies of an Abelian group $G$ we denote by $G^{(\omega)}$.

Let $\mathbf{u}=\left\{u_{n}\right\}_{n \in \omega}$ be a sequence in an Abelian group $G$. In general, no Hausdroff topology may exist in which $\mathbf{u}$ converges to zero. A very important question of whether there exists a Hausdorff group topology $\tau$ on $G$, such that $u_{n} \rightarrow 0$ in $(G, \tau)$, especially for the integers, has been studied by many authors; see Graev [1], 
Nienhuys [2], and others. Protasov and Zelenyuk [3] obtained a criterion that gives a complete answer to this question. Following [3], we say that a sequence $\mathbf{u}=\left\{u_{n}\right\}$ in an Abelian group $G$ is a $T$-sequence if there is a Hausdorff group topology on $G$ in which $u_{n}$ converges to zero. The finest group topology with this property we denote by $\tau_{\mathbf{u}}$.

The counterpart of the above question for precompact group topologies on $\mathbb{Z}$ is studied by Raczkowski [4]. Following [5,6] and motivated by [4], we say that a sequence $\mathbf{u}=\left\{u_{n}\right\}$ is a $T B$-sequence in an Abelian group $G$ if there is a precompact Hausdorff group topology on $G$ in which $u_{n}$ converges to zero. For a $T B$-sequence $\mathbf{u}$, we denote by $\tau_{b \mathbf{u}}$ the finest precompact group topology on $G$ in which $\mathbf{u}$ converges to zero. Clearly, every $T B$-sequence is a $T$-sequence, but in general, the converse assertion does not hold.

While it is quite hard to check whether a given sequence is a $T$-sequence (see, for example, [3,7-10]), the case of TB-sequences is much simpler. Let $X$ be an Abelian topological group and $\mathbf{u}=\left\{u_{n}\right\}$ be a sequence in its dual group $X^{\wedge}$. Following [11], set:

$$
s_{\mathbf{u}}(X)=\left\{x \in X:\left(u_{n}, x\right) \rightarrow 1\right\} .
$$

In [5], the following simple criterion to be a $T B$-sequence was obtained:

Fact 1 ([5]). A sequence $\mathbf{u}$ in a (discrete) Abelian group $G$ is a TB-sequence if and only if the subgroup $s_{\mathbf{u}}(X)$ of the (compact) dual $X=G^{\wedge}$ is dense.

Motivated by Fact 1, Dikranjan et al. [11] introduced the following notion related to subgroups of the form $s_{\mathbf{u}}(X)$ of a compact Abelian group $X$ :

Definition 2 ([11]). Let $H$ be a subgroup of a compact Abelian group $X$ and $\mathbf{u}=\left\{u_{n}\right\}$ be a sequence in $\widehat{X}$. If $H=s_{\mathbf{u}}(X)$, we say that $\mathbf{u}$ characterizes $H$ and that $H$ is characterized (by $\mathbf{u}$ ).

Note that for the torus $\mathbb{T}$, this notion was already defined in [12]. Characterized subgroups have been studied by many authors; see, for example, [11-16]. In particular, the main theorem of [15] (see also [14]) asserts that every countable subgroup of a compact metrizable Abelian group is characterized. It is natural to ask whether a closed subgroup of a compact Abelian group is characterized. The following easy criterion is given in [13]:

Fact 3 ([13]). A closed subgroup $H$ of a compact Abelian group $X$ is characterized if and only if $H$ is a $G_{\delta}$-subgroup. In particular, $X / H$ is metrizable, and the annihilator $H^{\perp}$ of $H$ is countable. 
The next fact follows easily from Definition 2:

Fact 4 ([17], see also [13]). Every characterized subgroup H of a compact Abelian group X is an $F_{\sigma \delta}$-subgroup of $X$, and hence, $H$ is a Borel subset of $X$.

Facts 3 and 4 inspired in [13] the study of the Borel hierarchy of characterized subgroups of compact Abelian groups. For a compact Abelian group $X$, denote by $\operatorname{Char}(X)$ (respectively, $\mathrm{SF}_{\sigma}(X), \mathrm{SF}_{\sigma \delta}(X)$ and $\mathrm{SG}_{\delta}(X)$ ) the set of all characterized subgroups (respectively, $F_{\sigma}$-subgroups, $F_{\sigma \delta}$-subgroups and $G_{\delta}$-subgroups) of $X$. The next fact is Theorem $\mathrm{E}$ in [13]:

Fact 5 ([13]). For every infinite compact Abelian group $X$, the following inclusions hold:

$$
\mathrm{SG}_{\delta}(X) \varsubsetneqq \operatorname{Char}(X) \varsubsetneqq \mathrm{SF}_{\sigma \delta}(X) \text { and } \mathrm{SF}_{\sigma}(X) \nsubseteq \mathrm{Char}(X) \text {. }
$$

If in addition $X$ has finite exponent, then:

$$
\operatorname{Char}(X) \varsubsetneqq \mathrm{SF}_{\sigma}(X) .
$$

The inclusion Equation (1) inspired the following question:

Question 6 (Problem 3.3 in [13]). Does there exist a compact Abelian group X of infinite exponent all of whose characterized subgroups are $F_{\sigma}$-subsets of $X$ ?

Main results: It is important to emphasize that there is no restriction on the sequence $\mathbf{u}$ in Definition 2. If a characterized subgroup $H$ of a compact Abelian group $X$ is dense, then, by Fact 1 , a characterizing sequence is also a $T B$-sequence. However, if $H$ is not dense, we cannot expect in general that a characterizing sequence of $H$ is a $T$-sequence. Thus, it is natural to ask:

Question 7. For which characterized subgroups of compact Abelian groups can one find characterizing sequences that are also T-sequences?

This question is of independent interest, because every $T$-sequence $\mathbf{u}$ naturally defines the group topology $\tau_{\mathbf{u}}$ satisfying the following dual property:

Fact 8 ([18]). Let $H$ be a subgroup of an infinite compact Abelian group X characterized by a T-sequence $\mathbf{u}$. Then, $\left(\widehat{X}, \tau_{\mathbf{u}}\right)^{\wedge}=H\left(=s_{\mathbf{u}}(X)\right)$ and $\mathbf{n}\left(\widehat{X}, \tau_{\mathbf{u}}\right)=H^{\perp}$ algebraically.

This motivates us to introduce the following notion: 
Definition 9. Let $H$ be a subgroup of a compact Abelian group $X$. We say that $H$ is a T-characterized subgroup of $X$ if there exists a T-sequence $\mathbf{u}=\left\{u_{n}\right\}_{n \in \omega}$ in $\widehat{X}$, such that $H=s_{\mathbf{u}}(X)$.

Denote by $\operatorname{Char}_{T}(X)$ the set of all T-characterized subgroups of a compact Abelian group $X$. Clearly, $\operatorname{Char}_{T}(X) \subseteq \operatorname{Char}(X)$. Hence, if a T-characterized subgroup $H$ of $X$ is closed, it is a $G_{\delta}$-subgroup of $X$ by Fact 3 . Note also that $X$ is $T$-characterized by the zero sequence.

The main goal of the article is to obtain a complete description of closed T-characterized subgroups (see Theorem 10) and to study the Borel hierarchy of $T$-characterized subgroups (see Theorem 18) of compact Abelian groups. In particular, we obtain a complete answer to Question 7 for closed characterized subgroups and give a negative answer to Question 6.

Note that, if a compact Abelian group $X$ is finite, then every $T$-sequence $\mathbf{u}$ in $\widehat{X}$ is eventually equal to zero. Hence, $s_{\mathbf{u}}(X)=X$. Thus, $X$ is the unique $T$-characterized subgroup of $X$. Therefore, in what follows, we shall consider only infinite compact groups.

The following theorem describes all closed subgroups of compact Abelian groups that are $T$-characterized.

Theorem 10. Let $H$ be a proper closed subgroup of an infinite compact Abelian group X. Then, the following assertions are equivalent:

(1) $H$ is a T-characterized subgroup of $X$;

(2) $H$ is a $G_{\delta}$-subgroup of $X$, and the countable group $H^{\perp}$ admits a Hausdorff MinAP group topology;

(3) $H$ is a $G_{\delta}$-subgroup of $X$ and one of the following holds:

(a) $H^{\perp}$ has infinite exponent;

(b) $H^{\perp}$ has finite exponent and contains a subgroup that is isomorphic to $\mathbb{Z}\left(\exp \left(H^{\perp}\right)\right)^{(\omega)}$.

Corollary 11. Let $X$ be an infinite compact metrizable Abelian group. Then, the trivial subgroup $H=\{0\}$ is T-characterized if and only if $\widehat{X}$ admits a Hausdorff MinAP group topology.

As an immediate corollary of Fact 3 and Theorem 10, we obtain a complete answer to Question 7 for closed characterized subgroups.

Corollary 12. A proper closed characterized subgroup $H$ of an infinite compact Abelian group $X$ is T-characterized if and only if $H^{\perp}$ admits a Hausdorff MinAP group topology. 
If $H$ is an open proper subgroup of $X$, then $H^{\perp}$ is non-trivial and finite. Thus, every Hausdorff group topology on $H^{\perp}$ is discrete. Taking into account Fact 3, we obtain:

Corollary 13. Every open proper subgroup $H$ of an infinite compact Abelian group $X$ is a characterized non-T-characterized subgroup of $X$.

Nevertheless (see Example 1 below), there is a compact metrizable Abelian group $X$ with a countable $T$-characterized subgroup $H$, such that its closure $\bar{H}$ is open. Thus, it may happen that the closure of a $T$-characterized subgroup is not $T$-characterized.

It is natural to ask for which compact Abelian groups all of their closed $G_{\delta}$-subgroups are $T$-characterized. The next theorem gives a complete answer to this question.

Theorem 14. Let $X$ be an infinite compact Abelian group. The following assertions are equivalent:

(1) All closed $G_{\delta}$-subgroups of $X$ are $T$-characterized;

(2) $X$ is connected.

By Corollary 2.8 of [13], the trivial subgroup $H=\{0\}$ of a compact Abelian group $X$ is a $G_{\delta}$-subgroup if and only if $X$ is metrizable. Therefore, we obtain:

Corollary 15. All closed subgroups of an infinite compact Abelian group $X$ are $T$-characterized if and only if $X$ is metrizable and connected.

Theorems 10 and 14 are proven in Section 2.

In the next theorem, we give a negative answer to Question 6:

Theorem 16. Every compact Abelian group of infinite exponent has a dense T-characterized subgroup, which is not an $F_{\sigma}$-subgroup.

As a corollary of the inclusion Equation (1) and Theorem 16, we obtain:

Corollary 17. For an infinite compact Abelian group $X$, the following assertions are equivalent:

(i) X has finite exponent;

(ii) every characterized subgroup of $X$ is an $F_{\sigma}$-subgroup;

(iii) every $T$-characterized subgroup of $X$ is an $F_{\sigma}$-subgroup.

Therefore, $\operatorname{Char}(X) \subseteq \mathrm{SF}_{\sigma}(X)$ if and only if $X$ has finite exponent. 
In the next theorem, we summarize the obtained results about the Borel hierarchy of $T$-characterized subgroups of compact Abelian groups.

Theorem 18. Let $X$ be an infinite compact Abelian group X. Then:

(1) $\operatorname{Char}_{T}(X) \varsubsetneqq \operatorname{SF}_{\sigma \delta}(X)$;

(2) $\mathrm{SG}_{\delta}(X) \cap \mathrm{Char}_{T}(X) \varsubsetneqq \operatorname{Char}_{T}(X)$;

(3) $\mathrm{SG}_{\delta}(X) \subseteq \operatorname{Char}_{T}(X)$ if and only if $X$ is connected;

(4) $\operatorname{Char}_{T}(X) \cap \mathrm{SF}_{\sigma}(X) \varsubsetneqq \mathrm{SF}_{\sigma}(X)$;

(5) $\operatorname{Char}_{T}(X) \subseteq \mathrm{SF}_{\sigma}(X)$ if and only if $X$ has finite exponent.

We prove Theorems 16 and 18 in Section 3.

The notions of $\mathfrak{g}$-closed and $\mathfrak{g}$-dense subgroups of a compact Abelian group $X$ were defined in [11]. In the last section of the paper, in analogy to these notions, we define $\mathfrak{g}_{T}$-closed and $\mathfrak{g}_{T}$-dense subgroups of $X$. In particular, we show that every $\mathfrak{g}_{T}$-dense subgroup of a compact Abelian group $X$ is dense if and only if $X$ is connected (see Theorem 37).

\section{The Proofs of Theorems 10 and $\mathbf{1 4}$}

The subgroup of a group $G$ generated by a subset $A$ we denote by $\langle A\rangle$.

Recall that a subgroup $H$ of an Abelian topological group $X$ is called dually closed in $X$ if for every $x \in X \backslash H$, there exists a character $\chi \in H^{\perp}$, such that $(\chi, x) \neq 1$. $H$ is called dually embedded in $X$ if every character of $H$ can be extended to a character of $X$. Every open subgroup of $X$ is dually closed and dually embedded in $X$ by Lemma 3 of [19].

The next notion generalizes the notion of the maximal extension in the class of all compact Abelian groups introduced in [20].

Definition 19. Let $\mathcal{G}$ be an arbitrary class of topological groups. Let $(G, \tau) \in \mathcal{G}$ and $H$ be a subgroup of $G$. The group $(G, \tau)$ is called a maximal extension of $\left(H,\left.\tau\right|_{H}\right)$ in the class $\mathcal{G}$ if $\sigma \leq \tau$ for every group topology on $G$, such that $\left.\sigma\right|_{H}=\left.\tau\right|_{H}$ and $(G, \sigma) \in \mathcal{G}$.

Clearly, the maximal extension is unique if it exists. Note that in Definition 19, we do not assume that $\left(H,\left.\tau\right|_{H}\right)$ belongs to the class $\mathcal{G}$.

If $H$ is a subgroup of an Abelian group $G$ and $\mathbf{u}$ is a $T$-sequence (respectively, a $T B$-sequence) in $H$, we denote by $\tau_{\mathbf{u}}(H)$ (respectively, $\tau_{b \mathbf{u}}(H)$ ) the finest (respectively, precompact) group topology on $H$ generated by $\mathbf{u}$. We use the following easy corollary of the definition of $T$-sequences.

Lemma 20. For a sequence $\mathbf{u}$ in an Abelian group $G$, the following assertions are equivalent: 
(1) $\mathbf{u}$ is a T-sequence in $G$;

(2) $\mathbf{u}$ is a $T$-sequence in every subgroup of $G$ containing $\langle\mathbf{u}\rangle$;

(3) $\mathbf{u}$ is a T-sequence in $\langle\mathbf{u}\rangle$.

In this case, $\langle\mathbf{u}\rangle$ is open in $\tau_{\mathbf{u}}$ (and hence, $\langle\mathbf{u}\rangle$ is dually closed and dually embedded in $\left.\left(G, \tau_{\mathbf{u}}\right)\right)$, and $\left(G, \tau_{\mathbf{u}}\right)$ is the maximal extension of $\left(\langle\mathbf{u}\rangle, \tau_{\mathbf{u}}(\langle\mathbf{u}\rangle)\right.$ in the class TAG of all Abelian topological groups.

Proof. Evidently, (1) implies (2) and (2) implies (3). Let $\mathbf{u}$ be a $T$-sequence in $\langle\mathbf{u}\rangle$. Let $\tau$ be the topology on $G$ whose base is all translationsof $\tau_{\mathbf{u}}(\langle\mathbf{u}\rangle)$-open sets. Clearly, $\mathbf{u}$ converges to zero in $\tau$. Thus, $\mathbf{u}$ is a $T$-sequence in $G$. Therefore, (3) implies (1).

Let us prove the last assertion. By the definition of $\tau_{\mathbf{u}}$, we have also $\tau \leq \tau_{\mathbf{u}}$, and hence, $\left.\tau\right|_{\langle\mathbf{u}\rangle}=\tau_{\mathbf{u}}(\langle\mathbf{u}\rangle) \leq\left.\tau_{\mathbf{u}}\right|_{\langle\mathbf{u}\rangle}$. Thus, $\langle\mathbf{u}\rangle$ is open in $\tau_{\mathbf{u}}$, and hence, it is dually closed and dually embedded in $\left(G, \tau_{\mathbf{u}}\right)$ by [19] (Lemma 3.3). On the other hand, $\left.\tau_{\mathbf{u}}\right|_{\langle\mathbf{u}\rangle} \leq \tau_{\mathbf{u}}(\langle\mathbf{u}\rangle)=\left.\tau\right|_{\langle\mathbf{u}\rangle}$ by the definition of $\tau_{\mathbf{u}}(\langle\mathbf{u}\rangle)$. Therefore, $\tau_{\mathbf{u}}$ is an extension of $\tau_{\mathbf{u}}(\langle\mathbf{u}\rangle)$. Now, clearly, $\tau=\tau_{\mathbf{u}}$, and $\left(G, \tau_{\mathbf{u}}\right)$ is the maximal extension of $\left(\langle\mathbf{u}\rangle, \tau_{\mathbf{u}}(\langle\mathbf{u}\rangle)\right.$ in the class TAG.

For TB-sequences, we have the following:

Lemma 21. For a sequence $\mathbf{u}$ in an Abelian group $G$, the following assertions are equivalent:

(1) $\mathbf{u}$ is a TB-sequence in $G$;

(2) $\mathbf{u}$ is a TB-sequence in every subgroup of $G$ containing $\langle\mathbf{u}\rangle$;

(3) $\mathbf{u}$ is a TB-sequence in $\langle\mathbf{u}\rangle$.

In this case, the subgroup $\langle\mathbf{u}\rangle$ is dually closed and dually embedded in $\left(G, \tau_{b \mathbf{u}}\right)$, and $\left(G, \tau_{b \mathbf{u}}\right)$ is the maximal extension of $\left(\langle\mathbf{u}\rangle, \tau_{b \mathbf{u}}(\langle\mathbf{u}\rangle)\right)$ in the class of all precompact Abelian groups.

Proof. Evidently, (1) implies (2) and (2) implies (3). Let $\mathbf{u}$ be a TB-sequence in $\langle\mathbf{u}\rangle$. Then, $\left(\langle\mathbf{u}\rangle, \tau_{b \mathbf{u}}(\langle\mathbf{u}\rangle)\right)^{\wedge}$ separates the points of $\langle\mathbf{u}\rangle$. Let $\tau$ be the topology on $G$ whose base is all translations of $\tau_{b \mathbf{u}}(\langle\mathbf{u}\rangle)$-open sets. Then, $\left(\langle\mathbf{u}\rangle, \tau_{b \mathbf{u}}(\langle\mathbf{u}\rangle)\right)$ is an open subgroup of $(G, \tau)$. It is easy to see that $(G, \tau)^{\wedge}$ separates the points of $G$. Since $\mathbf{u}$ converges to zero in $\tau$, it also converges to zero in $\tau^{+}$, where $\tau^{+}$is the Bohr topology of $(G, \tau)$. Thus, $\mathbf{u}$ is a TB-sequence in $G$. Therefore, (3) implies (1).

The last assertion follows from Proposition 1.8 and Lemma 3.6 in [20].

For a sequence $\mathbf{u}=\left\{u_{n}\right\}_{n \in \omega}$ of characters of a compact Abelian group $X$, set:

$$
K_{\mathbf{u}}=\bigcap_{n \in \omega} \operatorname{ker}\left(u_{n}\right)
$$

The following assertions is proven in [13]: 
Fact 22 (Lemma 2.2(i) of [13]). For every sequence $\mathbf{u}=\left\{u_{n}\right\}_{n \in \omega}$ of characters of a compact Abelian group $X$, the subgroup $K_{\mathbf{u}}$ is a closed $G_{\delta}$-subgroup of $X$ and $K_{\mathbf{u}}=\langle\mathbf{u}\rangle^{\perp}$.

The next two lemmas are natural analogues of Lemmas 2.2(ii) and 2.6 of [13].

Lemma 23. Let $X$ be a compact Abelian group and $\mathbf{u}=\left\{u_{n}\right\}_{n \in \omega}$ be a T-sequence in $\widehat{X}$. Then, $s_{\mathbf{u}}(X) / K_{\mathbf{u}}$ is a T-characterized subgroup of $X / K_{\mathbf{u}}$.

Proof. Set $H:=s_{\mathbf{u}}(X)$ and $K:=K_{\mathbf{u}}$. Let $q: X \rightarrow X / K$ be the quotient map. Then, the adjoint homomorphism $q^{\wedge}$ is an isomorphism from $(X / K)^{\wedge}$ onto $K^{\perp}$ in $X^{\wedge}$. For every $n \in \omega$, define the character $\widetilde{u}_{n}$ of $X / K$ as follows: $\left(\tilde{u}_{n}, q(x)\right)=\left(u_{n}, x\right)\left(\widetilde{u}_{n}\right.$ is well-defined, since $\left.K \subseteq \operatorname{ker}\left(u_{n}\right)\right)$. Then, $\widetilde{\mathbf{u}}=\left\{\widetilde{u}_{n}\right\}_{n \in \omega}$ is a sequence of characters of $X / K$, such that $q^{\wedge}\left(\widetilde{u}_{n}\right)=u_{n}$. Since $\mathbf{u} \subset K^{\perp}, \mathbf{u}$ is a $T$-sequence in $K^{\perp}$ by Lemma 20 . Hence, $\widetilde{\mathbf{u}}$ is a $T$-sequence in $(X / K)^{\wedge}$ because $q^{\wedge}$ is an isomorphism.

We claim that $H / K=s_{\widetilde{\mathbf{u}}}(X / K)$. Indeed, for every $h+K \in H / K$, by definition, we have $\left(\widetilde{u}_{n}, h+K\right)=\left(u_{n}, h\right) \rightarrow 1$. Thus, $H / K \subseteq s_{\widetilde{\mathbf{u}}}(X / K)$. If $x+K \in s_{\widetilde{\mathbf{u}}}(X / K)$, then $\left(\widetilde{u}_{n}, x+K\right)=\left(u_{n}, x\right) \rightarrow 1$. This yields $x \in H$. Thus, $x+K \in H / K$.

Let $\mathbf{u}=\left\{u_{n}\right\}_{n \in \omega}$ be a $T$-sequence in an Abelian group $G$. For every natural number $m$, set $\mathbf{u}_{m}=\left\{u_{n}\right\}_{n \geq m}$. Clearly, $\mathbf{u}_{m}$ is a $T$-sequence in $G, \tau_{\mathbf{u}}=\tau_{\mathbf{u}_{m}}$ and $s_{\mathbf{u}}(X)=s_{\mathbf{u}_{m}}(X)$ for every natural number $m$.

Lemma 24. Let $K$ be a closed subgroup of a compact Abelian group $X$ and $q: X \rightarrow X / K$ be the quotient map. Then, $\widetilde{H}$ is a T-characterized subgroup of $X / K$ if and only if $q^{-1}(\widetilde{H})$ is a $T$-characterized subgroup of $X$.

Proof. Let $\widetilde{H}$ be a $T$-characterized subgroup of $X / K$, and let a $T$-sequence

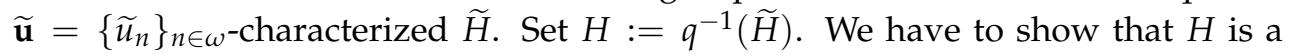
$T$-characterized subgroup of $X$.

Note that the adjoint homomorphism $q^{\wedge}$ is an isomorphism from $(X / K)^{\wedge}$ onto $K^{\perp}$ in $X^{\wedge}$. Set $\mathbf{u}=\left\{u_{n}\right\}_{n \in \omega}$, where $u_{n}=q^{\wedge}\left(\widetilde{u}_{n}\right)$. Since $q^{\wedge}$ is injective, $\mathbf{u}$ is a $T$-sequence in $K^{\perp}$. By Lemma $20, \mathbf{u}$ is a T-sequence in $\widehat{X}$. Therefore, it is enough to show that $H=s_{\mathbf{u}}(X)$. This follows from the following chain of equivalences. By definition, $x \in s_{\mathbf{u}}(X)$ if and only if:

$$
\left(u_{n}, x\right) \rightarrow 1 \Leftrightarrow\left(\widetilde{u}_{n}, q(x)\right) \rightarrow 1 \Leftrightarrow q(x) \in \widetilde{H}=H / K \Leftrightarrow x \in H .
$$

The last equivalence is due to the inclusion $K \subseteq H$.

Conversely, let $H:=q^{-1}(\widetilde{H})$ be a $T$-characterized subgroup of $X$ and a

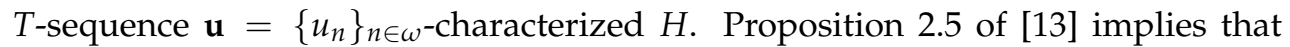
we can find $m \in \mathbb{N}$, such that $K \subseteq K_{\mathbf{u}_{m}}$. Therefore, taking into account that $H=s_{\mathbf{u}}(X)=s_{\mathbf{u}_{m}}(X)$ for every natural number $m$, without loss of generality, we 
can assume that $K \subseteq K_{\mathbf{u}}$. By Lemma $23, H / K_{\mathbf{u}}$ is a $T$-characterized subgroup of $X / K_{\mathbf{u}}$. Denote by $q_{u}$ the quotient homomorphism from $X / K$ onto $X / K_{\mathbf{u}}$. Then, $\widetilde{H}=q_{u}^{-1}\left(H / K_{\mathbf{u}}\right)$ is $T$-characterized in $X / K$ by the previous paragraph of the proof.

The next theorem is an analogue of Theorem B of [13], and it reduces the study of $T$-characterized subgroups of compact Abelian groups to the study of $T$-characterized ones of compact Abelian metrizable groups:

Theorem 25. A subgroup $H$ of a compact Abelian group $X$ is $T$-characterized if and only if $H$ contains a closed $G_{\delta}$-subgroup $K$ of $X$, such that $H / K$ is a $T$-characterized subgroup of the compact metrizable group $X / K$.

Proof. Let $H$ be $T$-characterized in $X$ by a $T$-sequence $\mathbf{u}=\left\{u_{n}\right\}_{n \in \omega}$ in $\widehat{X}$. Set $K:=K_{\mathbf{u}}$. Since $K$ is a closed $G_{\delta}$-subgroup of $X$ by Fact $22, X / K$ is metrizable. By Lemma $23, H / K$ is a $T$-characterized subgroup of $X / K$.

Conversely, let $H$ contain a closed $G_{\delta}$-subgroup $K$ of $X$, such that $H / K$ is a $T$-characterized subgroup of the compact metrizable group $X / K$. Then, $H$ is a $T$-characterized subgroup of $X$ by Lemma 24 .

As was noticed in [21] before Definition 2.33, for every $T$-sequence $\mathbf{u}$ in an infinite Abelian group $G$, the subgroup $\langle\mathbf{u}\rangle$ is open in $\left(G, \tau_{\mathbf{u}}\right)$ (see also Lemma 20), and hence, by Lemmas 1.4 and 2.2 of [22], the following sequences are exact:

$$
\begin{aligned}
& 0 \rightarrow\left(\langle\mathbf{u}\rangle, \tau_{\mathbf{u}}\right) \rightarrow\left(G, \tau_{\mathbf{u}}\right) \rightarrow G /\langle\mathbf{u}\rangle \rightarrow 0, \\
& 0 \rightarrow(G /\langle\mathbf{u}\rangle)^{\wedge} \rightarrow\left(G, \tau_{\mathbf{u}}\right)^{\wedge} \rightarrow\left(\langle\mathbf{u}\rangle,\left.\tau_{\mathbf{u}}\right|_{\langle\mathbf{u}\rangle}\right)^{\wedge} \rightarrow 0,
\end{aligned}
$$

where $(G /\langle\mathbf{u}\rangle)^{\wedge} \cong\langle\mathbf{u}\rangle^{\perp}$ is a compact subgroup of $\left(G, \tau_{\mathbf{u}}\right)^{\wedge}$ and $\left(\langle\mathbf{u}\rangle, \tau_{\mathbf{u}}\right)^{\wedge} \cong$ $\left(G, \tau_{\mathbf{u}}\right)^{\wedge} /\langle\mathbf{u}\rangle^{\perp}$.

Let $\mathbf{u}=\left\{u_{n}\right\}_{n \in \omega}$ be a $T$-sequence in an Abelian group G. It is known [10] that $\tau_{\mathbf{u}}$ is sequential, and hence, $\left(G, \tau_{\mathbf{u}}\right)$ is a $k$-space. Therefore, the natural homomorphism $\alpha:=\alpha_{\left(G, \tau_{\mathbf{u}}\right)}:\left(G, \tau_{\mathbf{u}}\right) \rightarrow\left(G, \tau_{\mathbf{u}}\right)^{\wedge \wedge}$ is continuous by [23] (5.12). Let us recall that $\left(G, \tau_{\mathbf{u}}\right)$ is MinAP if and only if $\left(G, \tau_{\mathbf{u}}\right)=\operatorname{ker}(\alpha)$.

To prove Theorem 10, we need the following:

Fact 26 ([16]). For each $T$-sequence $\mathbf{u}$ in a countably infinite Abelian group $G$, the group $\left(G, \tau_{\mathbf{u}}\right)^{\wedge}$ is Polish.

Now, we are in a position to prove Theorem 10.

Proof of Theorem 10. (1) $\Rightarrow$ (2) Let $H$ be a proper closed $T$-characterized subgroup of $X$ and a $T$-sequence $\mathbf{u}=\left\{u_{n}\right\}_{n \in \omega}$-characterized $H$. Since $H$ is also characterized, 
it is a $G_{\delta}$-subgroup of $X$ by Fact 3 . We have to show that $H^{\perp}$ admits a MinAP group topology.

Our idea of the proof is the following. Set $G:=\widehat{X}$. By Fact $8, H^{\perp}$ is the von Neumann radical of $\left(G, \tau_{\mathbf{u}}\right)$. Now, assume that we found another $T$-sequence $\mathbf{v}$ that characterizes $H$ and such that $\langle\mathbf{v}\rangle=H^{\perp}$ (maybe $\mathbf{v}=\mathbf{u}$ ). By Fact 8 , we have $\mathbf{n}\left(G, \tau_{\mathbf{v}}\right)=$ $H^{\perp}=\langle\mathbf{v}\rangle$. Lemma 20 implies that the subgroup $\left(\langle\mathbf{v}\rangle,\left.\tau_{\mathbf{v}}\right|_{\langle\mathbf{v}\rangle}\right)$ of $\left(G, \tau_{\mathbf{v}}\right)$ is open, and hence, it is dually closed and dually embedded in $\left(G, \tau_{\mathbf{v}}\right)$. Hence, $\mathbf{n}\left(\langle\mathbf{v}\rangle,\left.\tau_{\mathbf{v}}\right|_{\langle\mathbf{v}\rangle}\right)=$ $\mathbf{n}\left(G, \tau_{\mathbf{v}}\right)(=\langle\mathbf{v}\rangle)$ by Lemma 4 of [16]. Therefore, $\left(\langle\mathbf{v}\rangle,\left.\tau_{\mathbf{v}}\right|_{\langle\mathbf{v}\rangle}\right)$ is MinAP. Thus, $H^{\perp}=\langle\mathbf{v}\rangle$ admits a MinAP group topology, as desired.

We find such a $T$-sequence $\mathbf{v}$ in four steps (in fact, we show that $\mathbf{v}$ has the form $\mathbf{u}_{m}$ for some $m \in \mathbb{N}$ ).

Step 1 . Let $q: X \rightarrow X / K_{\mathbf{u}}$ be the quotient map. For every $n \in \omega$, define the character $\widetilde{u}_{n}$ of $X / K_{\mathbf{u}}$ by the equality $u_{n}=\widetilde{u}_{n} \circ q$ (this is possible since $K_{\mathbf{u}} \subseteq \operatorname{ker}\left(u_{n}\right)$ ). As was shown in the proof of Lemma 23 , the sequence $\widetilde{\mathbf{u}}=\left\{\widetilde{u}_{n}\right\}_{n \in \omega}$ is a $T$-sequence, which characterizes $H / K_{\mathbf{u}}$ in $X / K_{\mathbf{u}}$. Set $\widetilde{X}:=X / K_{\mathbf{u}}$ and $\widetilde{H}:=H / K_{\mathbf{u}}$. Therefore, $\widetilde{H}=s_{\widetilde{\mathbf{u}}}(\widetilde{X})$. By [24] (5.34 and 24.11) and since $K_{\mathbf{u}} \subseteq H$, we have:

$$
H^{\perp} \cong(X / H)^{\wedge} \cong(\widetilde{X} / \widetilde{H})^{\wedge} \cong \widetilde{H}^{\perp}
$$

By Fact $3, \widetilde{X}$ is metrizable. Hence, $\widetilde{H}$ is also compact and metrizable, and $\widetilde{G}:=\widehat{\widetilde{X}}$ is a countable Abelian group by [24] (24.15). Since $H$ is a proper closed subgroup of $X$, Equation (3) implies that $\widetilde{G}$ is non-zero.

We claim that $\widetilde{G}$ is countably infinite. Indeed, suppose for a contradiction that $\widetilde{G}$ is finite. Then, $X / K_{\mathbf{u}}=\widetilde{X}$ is also finite. Now, Fact 22 implies that $\langle\mathbf{u}\rangle$ is a finite subgroup of $G$. Since $\mathbf{u}$ is a $T$-sequence, $\mathbf{u}$ must be eventually equal to zero. Hence, $H=s_{\mathbf{u}}(X)=X$ is not a proper subgroup of $X$, a contradiction.

Step 2. We claim that there is a natural number $m$, such that the group $\left(\left\langle\widetilde{\mathbf{u}}_{m}\right\rangle,\left.\tau_{\widetilde{\mathbf{u}}}\right|_{\left\langle\widetilde{\mathbf{u}}_{m}\right\rangle}\right)=\left(\left\langle\widetilde{\mathbf{u}}_{m}\right\rangle,\left.\tau_{\widetilde{\mathbf{u}}_{m}}\right|_{\left\langle\widetilde{\mathbf{u}}_{m}\right\rangle}\right)$ is MinAP.

Indeed, since $\widetilde{G}$ is countably infinite, we can apply Fact 8 . Therefore, $\widetilde{H}=\left(\widetilde{G}, \tau_{\widetilde{\mathbf{u}}}\right)^{\wedge}$ algebraically. Since $\widetilde{H}$ and $\left(\widetilde{G}, \tau_{\widetilde{\mathbf{u}}}\right)^{\wedge}$ are Polish groups (see Fact 26 ), $\widetilde{H}$ and $\left(\widetilde{G}, \tau_{\widetilde{\mathbf{u}}}\right)^{\wedge}$ are topologically isomorphic by the uniqueness of the Polish group topology. Hence $\left(\widetilde{G}, \tau_{\widetilde{\mathbf{u}}}\right)^{\wedge} \wedge=\widetilde{H}^{\wedge}$ is discrete. As was noticed before the proof, the natural homomorphism $\widetilde{\alpha}:\left(\widetilde{G}, \tau_{\widetilde{\mathbf{u}}}\right) \rightarrow\left(\widetilde{G}, \tau_{\widetilde{\mathbf{u}}}\right)^{\wedge \wedge}$ is continuous. Since $\left(\widetilde{G}, \tau_{\widetilde{\mathbf{u}}}\right)^{\wedge \wedge}$ is discrete, we obtain that the von Neumann radical $\operatorname{ker}(\widetilde{\alpha})$ of $\left(\widetilde{G}, \tau_{\widetilde{\mathbf{u}}}\right)$ is open in $\tau_{\widetilde{\mathbf{u}}}$. Therefore, there exists a natural number $m$, such that $\widetilde{u}_{n} \in \operatorname{ker}(\widetilde{\alpha})$ for every $n \geq m$. Hence, $\left\langle\widetilde{\mathbf{u}}_{m}\right\rangle \subseteq \operatorname{ker}(\widetilde{\alpha})$. Lemma 20 implies that the subgroup $\left\langle\widetilde{\mathbf{u}}_{m}\right\rangle$ is open in $\left(\widetilde{G}, \tau_{\widetilde{\mathbf{u}}}\right)$, and hence, it is dually closed and dually embedded in $\left(\widetilde{G}, \tau_{\mathbf{u}}\right)$. Now, Lemma 4 of [16] yields $\left\langle\widetilde{\mathbf{u}}_{m}\right\rangle=\operatorname{ker}(\widetilde{\alpha})$, and $\left(\left\langle\widetilde{\mathbf{u}}_{m}\right\rangle,\left.\tau_{\widetilde{\mathbf{u}}}\right|_{\left\langle\widetilde{\mathbf{u}}_{m}\right\rangle}\right)$ is MinAP.

Step 3. Set $\mathbf{v}=\left\{v_{n}\right\}_{n \in \omega}$, where $v_{n}=u_{n+m}$ for every $n \in \omega$. Clearly, $\mathbf{v}$ is a $T$-sequence in $G$ characterizing $H, \tau_{\mathbf{u}}=\tau_{\mathbf{v}}$ and $K_{\mathbf{u}} \subseteq K_{\mathbf{v}}$. Let $t: X \rightarrow X / K_{\mathbf{v}}$ and 
$r: X / K_{\mathbf{u}} \rightarrow X / K_{\mathbf{v}}$ be the quotient maps. Analogously to Step 1 and the proof of Lemma 23, the sequence $\widetilde{\mathbf{v}}=\left\{\widetilde{v}_{n}\right\}_{n \in \omega}$ is a $T$-sequence in $\widehat{X / K_{\mathbf{v}}}$, which characterizes $H / K_{\mathbf{v}}$ in $X / K_{\mathbf{v}}$, where $v_{n}=\widetilde{v}_{n} \circ t$. Since $t=r \circ q$, we have:

$$
v_{n}=\widetilde{v}_{n} \circ t=t^{\wedge}\left(\widetilde{v}_{n}\right)=q^{\wedge}\left(r^{\wedge}\left(\widetilde{v}_{n}\right)\right)
$$

where $t^{\wedge}, r^{\wedge}$ and $q^{\wedge}$ are the adjoint homomorphisms to $t, r$ and $q$, respectively.

Since $q^{\wedge}$ and $r^{\wedge}$ are embeddings, we have $r^{\wedge}\left(\widetilde{v}_{n}\right)=\widetilde{u}_{n+m}$. In particular, $\langle\mathbf{v}\rangle \cong\langle\widetilde{\mathbf{v}}\rangle \cong\left\langle\widetilde{\mathbf{u}}_{m}\right\rangle$ and :

$$
\left(\left\langle\widetilde{\mathbf{u}}_{m}\right\rangle,\left.\tau_{\widetilde{\mathbf{u}}}\right|_{\left\langle\widetilde{\mathbf{u}}_{m}\right\rangle}\right)=\left(\left\langle\widetilde{\mathbf{u}}_{m}\right\rangle,\left.\tau_{\widetilde{\mathbf{u}}_{m}}\right|_{\left\langle\widetilde{\mathbf{u}}_{m}\right\rangle}\right) \cong\left(\langle\widetilde{\mathbf{v}}\rangle,\left.\tau_{\widetilde{\mathbf{v}}}\right|_{\langle\widetilde{\mathbf{v}}\rangle}\right) \cong\left(\langle\mathbf{v}\rangle,\left.\tau_{\mathbf{v}}\right|_{\langle\mathbf{v}\rangle}\right) .
$$

By Step 2, $\left(\left\langle\widetilde{\mathbf{u}}_{m}\right\rangle,\left.\tau_{\widetilde{\mathbf{u}}_{m}}\right|_{\left\langle\widetilde{\mathbf{u}}_{m}\right\rangle}\right)$ is MinAP. Hence, $\left(\langle\mathbf{v}\rangle,\left.\tau_{\mathbf{v}}\right|_{\langle\mathbf{v}\rangle}\right)$ is MinAP, as well.

Step 4. By the second exact sequence in Equation (2) applying to $\mathbf{v}$, Fact 8 , and since $\left(\langle\mathbf{v}\rangle,\left.\tau_{\mathbf{v}}\right|_{\langle\mathbf{v}\rangle}\right)$ is MinAP (by Step 3), we have $H=s_{\mathbf{v}}(X)=\left(G, \tau_{\mathbf{v}}\right)^{\wedge}=(G /\langle\mathbf{v}\rangle)^{\wedge}=$ $\langle\mathbf{v}\rangle^{\perp}$ algebraically. Thus, $H^{\perp}=\langle\mathbf{v}\rangle$, and hence, $H^{\perp}$ admits a MinAP group topology generated by the $T$-sequence $\mathbf{v}$.

$(2) \Rightarrow(1)$ : Since $H$ is a $G_{\delta}$-subgroup of $X, H$ is closed by [13] (Proposition 2.4) and $X / H$ is metrizable (due to the well-known fact that a compact group of countable pseudo-character is metrizable). Hence, $H^{\perp}=(X / H)^{\wedge}$ is countable. Since $H^{\perp}$ admits a MinAP group topology, $H^{\perp}$ must be countably infinite. By Theorem 3.8 of [9], $H^{\perp}$ admits a MinAP group topology generated by a $T$-sequence $\widetilde{\mathbf{u}}=\left\{\widetilde{u}_{n}\right\}_{n \in \omega}$. By Fact 8 , this means that $s_{\widetilde{\mathbf{u}}}(X / H)=\{0\}$. Let $q: X \rightarrow X / H$ be the quotient map. Set $u_{n}=\widetilde{u}_{n} \circ q=q^{\wedge}\left(\widetilde{u}_{n}\right)$. Since $q^{\wedge}$ is injective, $\mathbf{u}$ is a $T$-sequence in $\widehat{X}$ by Lemma 20 . We have to show that $H=s_{\mathbf{u}}(X)$. By definition, $x \in s_{\mathbf{u}}(X)$ if and only if:

$$
\left(u_{n}, x\right)=\left(\widetilde{u}_{n}, q(x)\right) \rightarrow 1 \Leftrightarrow q(x) \in s_{\widetilde{\mathbf{u}}}(X / H) \Leftrightarrow q(x)=0 \Leftrightarrow x \in H .
$$

(2) $\Leftrightarrow(3)$ follows from Theorem 3.8 of [9]. The theorem is proven.

Proof of Theorem 14. (1) $\Rightarrow(2)$ : Suppose for a contradiction that $X$ is not connected. Then, by [24] (24.25), the dual group $G=X^{\wedge}$ has a non-zero element $g$ of finite order. Then, the subgroup $H:=\langle g\rangle^{\perp}$ of $X$ has finite index. Hence, $H$ is an open subgroup of $X$. Thus, $H$ is not $T$-characterized by Corollary 13 . This contradiction shows that $X$ must be connected.

$(2) \Rightarrow(1)$ : Let $H$ be a proper $G_{\delta}$-subgroup of $X$. Then, $H$ is closed by [13] (Proposition 2.4), and $X / H$ is connected and non-zero. Hence, $H^{\perp} \cong(X / H)^{\wedge}$ is countably infinite and torsion free by [24] (24.25). Thus, $H^{\perp}$ has infinite exponent. Therefore, by Theorem 10, $H$ is $T$-characterized.

The next proposition is a simple corollary of Theorem B in [13]. 
Proposition 27. The closure $\bar{H}$ of a characterized (in particular, T-characterized) subgroup $H$ of a compact Abelian group $X$ is a characterized subgroup of $X$.

Proof. By Theorem B of [13], $H$ contains a compact $G_{\delta}$-subgroup $K$ of $X$. Then, $\bar{H}$ is also a $G_{\delta}$-subgroup of $X$. Thus, $\bar{H}$ is a characterized subgroup of $X$ by Theorem $B$ of [13].

In general, we cannot assert that the closure $\bar{H}$ of a $T$-characterized subgroup $H$ of a compact Abelian group $X$ is also $T$-characterized, as the next example shows.

Example 1. Let $X=\mathbb{Z}(2) \times \mathbb{T}$ and $G=\widehat{X}=\mathbb{Z}(2) \times \mathbb{Z}$. It is known (see the end of (1) in [7]) that there is a $T$-sequence $\mathbf{u}$ in $G$, such that the von Neumann radical $\mathbf{n}\left(G, \tau_{\mathbf{u}}\right)$ of $\left(G, \tau_{\mathbf{u}}\right)$ is $\mathbb{Z}(2) \times\{0\}$, the subgroup $H:=s_{\mathbf{u}}(X)$ is countable and $\bar{H}=\{0\} \times \mathbb{T}$. Therefore, the closure $\bar{H}$ of the countable $T$-characterized subgroup $H$ of $X$ is open. Thus, $\bar{H}$ is not $T$-characterized by Corollary 13 .

We do not know the answers to the following questions:

Problem 28. Let $H$ be a characterized subgroup of a compact Abelian group $X$, such that its closure $\bar{H}$ is $T$-characterized. Is $H$ a $T$-characterized subgroup of $X$ ?

Problem 29. Does there exists a metrizable Abelian compact group that has a countable non-T-characterized subgroup?

\section{The Proofs of Theorems $\mathbf{1 6}$ and $\mathbf{1 8}$}

Recall that a Borel subgroup $H$ of a Polish group $X$ is called polishable if there exists a Polish group topology $\tau$ on $H$, such that the inclusion map $i:(H, \tau) \rightarrow X$ is continuous. Let $H$ be a $T$-characterized subgroup of a compact metrizable Abelian group $X$ by a $T$-sequence $\mathbf{u}=\left\{u_{n}\right\}_{n \in \omega}$. Then, by [16] (Theorem 1 ), $H$ is polishable by the metric:

$$
\rho(x, y)=d(x, y)+\sup \left\{\left|\left(u_{n}, x\right)-\left(u_{n}, y\right)\right|, n \in \omega\right\},
$$

where $d$ is the initial metric on $X$. Clearly, the topology generated by the metric $\rho$ on $H$ is finer than the induced one from $X$.

To prove Theorem 16 we need the following three lemmas.

For a real number $x$, we write $[x]$ for the integral part of $x$ and $\|x\|$ for the distance from $x$ to the nearest integer. We also use the following inequality proven in [25]:

$$
\pi|\varphi| \leq\left|1-e^{2 \pi i \varphi}\right| \leq 2 \pi|\varphi|, \quad \varphi \in\left[-\frac{1}{2}, \frac{1}{2}\right) .
$$


Lemma 30. Let $\left\{a_{n}\right\}_{n \in \omega} \subset \mathbb{N}$ be such that $a_{n} \rightarrow \infty$ and $a_{n} \geq 2, n \in \omega$. Set $u_{n}=\prod_{k \leq n} a_{n}$ for every $n \in \omega$. Then, $\mathbf{u}=\left\{u_{n}\right\}_{n \in \omega}$ is a T-sequence in $X=\mathbb{T}$, and the T-characterized subgroup $H=s_{\mathbf{u}}(\mathbb{T})$ of $\mathbb{T}$ is a dense non- $F_{\sigma}$-subset of $\mathbb{T}$.

Proof. We consider the circle group $\mathbb{T}$ as $\mathbb{R} / \mathbb{Z}$ and write it additively. Therefore, $d(0, x)=\|x\|$ for every $x \in \mathbb{T}$. Recall that every $x \in \mathbb{T}$ has the unique representation in the form:

$$
x=\sum_{n=0}^{\infty} \frac{c_{n}}{u_{n}}
$$

where $0 \leq c_{n}<a_{n}$ and $c_{n} \neq a_{n}-1$ for infinitely many indices $n$.

It is known [26] (see also (12) in the proof of Lemma 1 of [25]) that $x$ with representation Equation (6) belongs to $H$ if and only if:

$$
\lim _{n \rightarrow \infty} \frac{c_{n}}{a_{n}}(\bmod 1)=0 .
$$

Hence, $H$ is a dense subgroup of $\mathbb{T}$. Thus, $\mathbf{u}$ is even a $T B$-sequence in $\mathbb{Z}$ by Fact 1 .

We have to show that $H$ is not an $F_{\sigma}$-subset of $\mathbb{T}$. Suppose for a contradiction that $H$ is an $F_{\sigma}$-subset of $\mathbb{T}$. Then, $H=\cup_{n \in \mathbb{N}} F_{n}$, where $F_{n}$ is a compact subset of $\mathbb{T}$ for every $n \in \mathbb{N}$. Since $H$ is a subgroup of $\mathbb{T}$, without loss of generality, we can assume that $F_{n}-F_{n} \subseteq F_{n+1}$. Since all $F_{n}$ are closed in $(H, \rho)$, as well, the Baire theorem implies that there are $0<\varepsilon<0.1$ and $m \in \mathbb{N}$, such that $F_{m} \supseteq\{x: \rho(0, x) \leq \varepsilon\}$.

Fix arbitrarily $l>0$, such that $\frac{2}{u_{l-1}}<\frac{\varepsilon}{20}$. For every natural number $k>l$, set:

$$
x_{k}:=\sum_{n=l}^{k} \frac{1}{u_{n}} \cdot\left[\frac{\left(a_{n}-1\right) \varepsilon}{20}\right] \text {. }
$$

Then, for every $k>l$, we have:

$$
x_{k}=\sum_{n=l}^{k} \frac{1}{u_{n}} \cdot\left[\frac{\left(a_{n}-1\right) \varepsilon}{20}\right]<\sum_{n=l}^{k} \frac{1}{u_{n-1}} \cdot \frac{\varepsilon}{20}<\frac{1}{u_{l-1}} \sum_{n=0}^{k-l} \frac{1}{2^{n}}<\frac{2}{u_{l-1}}<\frac{\varepsilon}{20}<\frac{1}{2} .
$$

This inequality and Equation (5) imply that:

$$
d\left(0, x_{k}\right)=\left\|x_{k}\right\|=x_{k}<\frac{\varepsilon}{20}, \text { for every } k>l .
$$

For every $s \in \omega$ and every natural number $k>l$, we estimate $\left|1-\left(u_{s}, x_{k}\right)\right|$ as follows. 
Case 1. Let $s<k$. Set $q=\max \{s+1, l\}$. By the definition of $x_{k}$, we have:

$$
\begin{aligned}
2 \pi\left[\left(u_{s} \cdot x_{k}\right)(\bmod 1)\right] & =2 \pi\left[u_{s} \sum_{n=l}^{k} \frac{1}{u_{n}} \cdot\left[\frac{\left(a_{n}-1\right) \varepsilon}{20}\right](\bmod 1)\right]<2 \pi \sum_{n=q}^{k} \frac{u_{s}}{u_{n}} \cdot \frac{\left(a_{n}-1\right) \varepsilon}{20} \\
& <\frac{\pi \varepsilon}{10}\left(1+\frac{1}{a_{s+1}}+\frac{1}{a_{s+1} a_{s+2}}+\frac{1}{a_{s+1} a_{s+2} a_{s+3}}+\ldots\right) \\
& <\frac{\pi \varepsilon}{10}\left(1+\frac{1}{2}+\frac{1}{2^{2}}+\frac{1}{2^{3}}+\ldots\right)=\frac{\pi \varepsilon}{10} \cdot 2<\frac{2 \varepsilon}{3}<\frac{1}{2} .
\end{aligned}
$$

This inequality and Equation (5) imply:

$$
\left|1-\left(u_{s}, x_{k}\right)\right|=\left|1-\exp \left\{2 \pi i \cdot\left[\left(u_{s} \cdot x_{k}\right)(\bmod 1)\right]\right\}\right|<\frac{2 \varepsilon}{3} .
$$

Case 2. Let $s \geq k$. By the definition of $x_{k}$, we have:

$$
\left|1-\left(u_{s}, x_{k}\right)\right|=0 .
$$

In particular, Equation (10) implies that $x_{k} \in H$ for every $k>l$.

Now, for every $k>l$, Equations (4) and (8)-(10) imply:

$$
\rho\left(0, x_{k}\right)<\frac{\varepsilon}{20}+\frac{2 \varepsilon}{3}<\varepsilon .
$$

Thus, $x_{k} \in F_{m}$ for every natural number $k>l$. Clearly,

$$
x_{k} \rightarrow x:=\sum_{n=l}^{\infty} \frac{1}{u_{n}} \cdot\left[\frac{\left(a_{n}-1\right) \varepsilon}{20}\right] \quad \text { in } \mathbb{T} .
$$

Since $F_{m}$ is a compact subset of $\mathbb{T}$, we have $x \in F_{m}$. Hence, $x \in H$. On the other hand, we have:

$$
\lim _{n \rightarrow \infty} \frac{1}{a_{n}} \cdot\left[\frac{\left(a_{n}-1\right) \varepsilon}{20}\right](\bmod 1)=\frac{\varepsilon}{20} \neq 0 .
$$

Therefore, Equation (7) implies that $x \notin H$. This contradiction shows that $H=s_{\mathbf{u}}(\mathbb{T})$ is not an $F_{\sigma}$-subset of $\mathbb{T}$.

For a prime number $p$, the group $\mathbb{Z}\left(p^{\infty}\right)$ is regarded as the collection of fractions $m / p^{n} \in[0,1)$. Let $\Delta_{p}$ be the compact group of $p$-adic integers. It is well known that $\widehat{\Delta_{p}}=\mathbb{Z}\left(p^{\infty}\right)$. 
Lemma 31. Let $X=\Delta_{p}$. For an increasing sequence of natural numbers $0<n_{0}<n_{1}<$ $\ldots$, such that $n_{k+1}-n_{k} \rightarrow \infty$, set:

$$
u_{k}=\frac{1}{p^{n_{k}+1}} \in \mathbb{Z}\left(p^{\infty}\right)
$$

Then, the sequence $\mathbf{u}=\left\{u_{k}\right\}_{k \in \omega}$ is a T-sequence in $\mathbb{Z}\left(p^{\infty}\right)$, and the T-characterized subgroup $H=s_{\mathbf{u}}\left(\Delta_{p}\right)$ is a dense non- $F_{\sigma}$-subset of $\Delta_{p}$.

Proof. Let $\omega=\left(a_{n}\right)_{n \in \omega} \in \Delta_{p}$, where $0 \leq a_{n}<p$ for every $n \in \omega$. Recall that, for every $k \in \omega,[24]$ (25.2) implies:

$$
\left(u_{k}, \omega\right)=\exp \left\{\frac{2 \pi i}{p^{n_{k}+1}}\left(a_{0}+p a_{1}+\cdots+p^{n_{k}} a_{n_{k}}\right)\right\} .
$$

Further, by [24] (10.4), if $\omega \neq 0$, then $d(0, \omega)=2^{-n}$, where $n$ is the minimal index, such that $a_{n} \neq 0$.

Following [27] (2.2), for every $\omega=\left(a_{n}\right) \in \Delta_{p}$ and every natural number $k>1$, set:

$$
m_{k}=m_{k}(\omega)=\max \left\{j_{k}, n_{k-1}\right\}
$$

where:

$$
j_{k}=n_{k} \text { if } 0<a_{n_{k}}<p-1
$$

and otherwise:

$$
j_{k}=\min \left\{j: \text { either } a_{s}=0 \text { for } j<s \leq n_{k} \text { or } a_{s}=p-1 \text { for } j<s \leq n_{k}\right\}
$$

In [27] (2.2), it is shown that:

$$
\omega \in s_{\mathbf{u}}\left(\Delta_{p}\right) \text { if and only if } n_{k}-m_{k} \rightarrow \infty
$$

Therefore, $H:=s_{\mathbf{u}}\left(\Delta_{p}\right)$ contains the identity $\mathbf{1}=(1,0,0, \ldots)$ of $\Delta_{p}$. By [24] (Remark 10.6), $\langle\mathbf{1}\rangle$ is dense in $\Delta_{p}$. Hence, $H$ is dense in $\Delta_{p}$, as well. Now, Fact 1 implies that $\mathbf{u}$ is a $T$-sequence in $\mathbb{Z}\left(p^{\infty}\right)$.

We have to show that $H$ is not an $F_{\sigma}$-subset of $\Delta_{p}$. Suppose for a contradiction that $H=\cup_{n \in \mathbb{N}} F_{n}$ is an $F_{\sigma}$-subset of $\Delta_{p}$, where $F_{n}$ is a compact subset of $\Delta_{p}$ for every $n \in \mathbb{N}$. Since $H$ is a subgroup of $\Delta_{p}$, without loss of generality, we can assume that $F_{n}-F_{n} \subseteq F_{n+1}$. Since all $F_{n}$ are closed in $(H, \rho)$, as well, the Baire theorem implies that there are $0<\varepsilon<0.1$ and $m \in \mathbb{N}$, such that $F_{m} \supseteq\{x: \rho(0, x) \leq \varepsilon\}$. 
Fix a natural number $s$, such that $\frac{1}{2^{s}}<\frac{\varepsilon}{20}$. Choose a natural number $l>s$, such that, for every natural number $w \geq l$, we have:

$$
n_{w+1}-n_{w}>s
$$

For every $r \in \mathbb{N}$, set:

$$
\omega_{r}:=\left(a_{n}^{r}\right), \text { where } a_{n}^{r}=\left\{\begin{array}{l}
1, \text { if } n=n_{l+i}-s \text { for some } 1 \leq i \leq r \\
0, \text { otherwise. }
\end{array}\right.
$$

Then, for every $r \in \mathbb{N}$, Equation (13) implies that $\omega_{r}$ is well defined and:

$$
d\left(0, \omega_{r}\right)=\frac{1}{2^{n_{l+1}-s}}<\frac{1}{2^{n_{l}}} \leq \frac{1}{2^{l}}<\frac{1}{2^{s}}<\frac{\varepsilon}{20} .
$$

Note that:

$$
1+p+\cdots+p^{k}=\frac{p^{k+1}-1}{p-1}<p^{k+1} .
$$

For every $k \in \omega$ and every $r \in \mathbb{N}$, we estimate $\left|1-\left(u_{k}, \omega_{r}\right)\right|$ as follows.

Case 1. Let $k \leq l$. By Equations (11) and (13) and the definition of $\omega_{r}$, we have:

$$
\left|1-\left(u_{k}, \omega_{r}\right)\right|=0
$$

Case 2. Let $l<k \leq l+r$. Then, Equation (15) yields:

$$
\frac{2 \pi}{p^{n_{k}+1}}\left|p^{n_{l+1}-s}+\cdots+p^{n_{k}-s}\right|<\frac{2 \pi}{p^{n_{k}+1}} \cdot p^{n_{k}-s+1}=\frac{2 \pi}{p^{s}} \leq \frac{2 \pi}{2^{s}}<\frac{\varepsilon}{2}<\frac{1}{2} .
$$

This inequality and the inequality Equations (5) and (11) imply:

$$
\left|1-\left(u_{k}, \omega_{r}\right)\right|=\left|1-\exp \left\{\frac{2 \pi i}{p^{n_{k}+1}}\left(p^{n_{l+1}-s}+\cdots+p^{n_{k}-s}\right)\right\}\right|<\frac{\varepsilon}{2} .
$$

Case 3. Let $l+r<k$. By Equation (15), we have:

$$
\begin{aligned}
\frac{2 \pi}{p^{n_{k}+1}}\left|p^{n_{l+1}-s}+\cdots+p^{n_{l+r}-s}\right| & <\frac{2 \pi}{p^{n_{k}+1}} \cdot p^{n_{l+r}-s+1} \\
& <\frac{2 \pi}{p^{n_{k}+1}} \cdot p^{n_{k}-s+1}=\frac{2 \pi}{p^{s}} \leq \frac{2 \pi}{2^{s}}<\frac{\varepsilon}{2} .
\end{aligned}
$$

These inequalities, Equations (5) and (11) immediately yield:

$$
\left|1-\left(u_{k}, \omega_{r}\right)\right|=\left|1-\exp \left\{\frac{2 \pi i}{p^{n_{k}+1}}\left(p^{n_{l+1}-s}+\cdots+p^{n_{l+r}-s}\right)\right\}\right|<\frac{\varepsilon}{2},
$$


and:

$$
\left|1-\left(u_{k}, \omega_{r}\right)\right|<\frac{2 \pi}{p^{n_{k}+1}} \cdot p^{n_{l+r}-s+1} \rightarrow 0, \text { as } k \rightarrow \infty .
$$

Therefore, Equation (19) implies that $\omega_{r} \in H$ for every $r \in \mathbb{N}$.

For every $r \in \mathbb{N}$, by Equations (4), (14) and (16)-(18), we have:

$$
\rho\left(0, \omega_{r}\right)=d\left(0, \omega_{r}\right)+\sup \left\{\left|1-\left(u_{k}, \omega_{r}\right)\right|, k \in \omega\right\}<\frac{\varepsilon}{20}+\frac{\varepsilon}{2}<\varepsilon .
$$

Thus, $\omega_{r} \in F_{m}$ for every $r \in \mathbb{N}$. Evidently,

$$
\omega_{r} \rightarrow \widetilde{\omega}=\left(\widetilde{a}_{n}\right) \text { in } \Delta_{p}, \text { where } \widetilde{a}_{n}=\left\{\begin{array}{l}
1, \text { if } n=n_{l+i}-s \text { for some } i \in \mathbb{N}, \\
0, \text { otherwise. }
\end{array}\right.
$$

Since $F_{m}$ is a compact subset of $\Delta_{p}$, we have $\widetilde{\omega} \in F_{m}$. Hence, $\widetilde{\omega} \in H$. On the other hand, it is clear that $m_{k}(\widetilde{\omega})=n_{k}-s$ for every $k \geq l+1$. Thus, for every $k \geq l+1$, $n_{k}-m_{k}(\widetilde{\omega})=s \nrightarrow \rightarrow \infty$. Now, Equation (12) implies that $\widetilde{\omega} \notin H$. This contradiction shows that $H$ is not an $F_{\sigma}$-subset of $\Delta_{p}$.

Lemma 32. Let $X=\prod_{n \in \omega} \mathbb{Z}\left(b_{n}\right)$, where $1<b_{0}<b_{1}<\ldots$ and $G:=\widehat{X}=\bigoplus_{n \in \omega} \mathbb{Z}\left(b_{n}\right)$. Set $\mathbf{u}=\left\{u_{n}\right\}_{n \in \omega}$, where $u_{n}=1 \in \mathbb{Z}\left(b_{n}\right)^{\wedge} \subset G$ for every $n \in \omega$. Then, $\mathbf{u}$ is a T-sequence in $G$, and the T-characterized subgroup $H=s_{\mathbf{u}}(X)$ is a dense non- $F_{\sigma}$-subset of $X$.

Proof. Set $H:=s_{\mathbf{u}}(X)$. In [27] (2.3), it is shown that:

$$
\omega=\left(a_{n}\right) \in s_{\mathbf{u}}(X) \text { if and only if }\left\|\frac{a_{n}}{b_{n}}\right\| \rightarrow 0 .
$$

Therefore, $\bigoplus_{n \in \omega} \mathbb{Z}\left(b_{n}\right) \subseteq H$. Thus, $H$ is dense in $X$. Now, Fact 1 implies that $\mathbf{u}$ is a $T$-sequence in $G$.

We have to show that $H$ is not an $F_{\sigma}$-subset of $X$. Suppose for a contradiction that $H=\cup_{n \in \mathbb{N}} F_{n}$ is an $F_{\sigma}$-subset of $X$, where $F_{n}$ is a compact subset of $X$ for every $n \in \mathbb{N}$. Since $H$ is a subgroup of $X$, without loss of generality, we can assume that $F_{n}-F_{n} \subseteq F_{n+1}$. Since all $F_{n}$ are closed in $(H, \rho)$, as well, the Baire theorem yields that there are $0<\varepsilon<0.1$ and $m \in \mathbb{N}$, such that $F_{m} \supseteq\{\omega \in X: \rho(0, \omega) \leq \varepsilon\}$.

Note that $d(0, \omega)=2^{-l}$, where $0 \neq \omega=\left(a_{n}\right)_{n \in \omega} \in X$ and $l$ is the minimal index, such that $a_{l} \neq 0$. Choose $l$, such that $2^{-l}<\varepsilon / 3$. For every natural number $k>l$, set:

$$
\omega_{k}:=\left(a_{n}^{k}\right), \text { where } a_{n}^{k}=\left\{\begin{array}{c}
{\left[\frac{\varepsilon b_{n}}{20}\right], \text { for every } n \text { such that } l \leq n \leq k,} \\
0, \text { if either } 1 \leq n<l \text { or } k<n .
\end{array}\right.
$$


Since $\left(u_{n}, \omega_{k}\right)=1$ for every $n>k$, we obtain that $\omega_{k} \in H$ for every $k>l$. For every $n \in \omega$, we have:

$$
2 \pi \cdot \frac{1}{b_{n}}\left[\frac{\varepsilon b_{n}}{20}\right]<\frac{2 \pi \varepsilon}{20}<\varepsilon<\frac{1}{2} .
$$

This inequality and the inequality Equations (4) and (5) imply:

$$
\begin{aligned}
\rho\left(0, \omega_{k}\right) & =d\left(0, \omega_{k}\right)+\sup \left\{\left|1-\left(u_{n}, \omega_{k}\right)\right|, n \in \omega\right\} \\
& \leq \frac{1}{2^{l}}+\max \left\{\left|1-\exp \left\{2 \pi i \frac{1}{b_{n}}\left[\frac{\varepsilon b_{n}}{20}\right]\right\}\right|, l \leq n \leq k\right\} \\
& \leq \frac{\varepsilon}{3}+2 \pi \cdot \max \left\{\frac{1}{b_{n}}\left[\frac{\varepsilon b_{n}}{20}\right], l \leq n \leq k\right\}<\frac{\varepsilon}{3}+\frac{2 \pi \varepsilon}{20}<\varepsilon .
\end{aligned}
$$

Thus, $\omega_{k} \in F_{m}$ for every natural number $k>l$. Evidently,

$$
\omega_{k} \rightarrow \widetilde{\omega}=\left(\widetilde{a}_{n}\right)_{n \in \omega} \text { in } X, \text { where } \widetilde{a}_{n}=\left\{\begin{array}{r}
0, \text { if } 0 \leq n<l, \\
{\left[\frac{\varepsilon b_{n}}{20}\right], \text { if } l \leq n .}
\end{array}\right.
$$

Since $F_{m}$ is a compact subset of $X$, we have $\widetilde{\omega} \in F_{m}$. Hence, $\widetilde{\omega} \in H$. On the other hand, since $b_{n} \rightarrow \infty$, we have:

$$
\lim _{n \rightarrow \infty}\left\|\frac{\widetilde{a}_{n}}{b_{n}}\right\|=\lim _{n \rightarrow \infty} \frac{1}{b_{n}}\left[\frac{\varepsilon b_{n}}{20}\right]=\frac{\varepsilon}{20} \neq 0 .
$$

Thus, $\widetilde{\omega} \notin H$ by Equation (20). This contradiction shows that $H$ is not an $F_{\sigma}$-subset of $X$.

Now, we are in a position to prove Theorems 16 and 18.

Proof of Theorem 16. Let $X$ be a compact Abelian group of infinite exponent. Then, $G:=\widehat{X}$ also has infinite exponent. It is well-known that $G$ contains a countably-infinite subgroup $S$ of one of the following form:

(a) $S \cong \mathbb{Z}$;

(b) $S \cong \mathbb{Z}\left(p^{\infty}\right)$;

(c) $S \cong \bigoplus_{n \in \omega} \mathbb{Z}\left(b_{n}\right)$, where $1<b_{0}<b_{1}<\ldots$

Fix such a subgroup $S$. Set $K=S^{\perp}$ and $Y=X / K \cong S_{d}^{\wedge}$, where $S_{d}$ denotes the group $S$ endowed with the discrete topology. Since $S$ is countable, $Y$ is metrizable. Hence, $\{0\}$ is a $G_{\delta}$-subgroup of $Y$. Thus, $K$ is a $G_{\delta}$-subgroup of $X$. Let $q: X \rightarrow Y$ be the quotient map. By Lemmas 30-32, the compact group $Y$ has a dense $T$-characterized subgroup $\widetilde{H}$, which is not an $F_{\sigma}$-subset of $Y$. Lemma 24 implies that $H:=q^{-1}(\widetilde{H})$ is a 
dense $T$-characterized subgroup of $X$. Since the continuous image of an $F_{\sigma}$-subset of a compact group is an $F_{\sigma}$-subset, as well, we obtain that $H$ is not an $F_{\sigma}$-subset of $X$. Thus, the subgroup $H$ of $X$ is $T$-characterized, but it is not an $F_{\sigma}$-subset of $X$. The theorem is proven.

Proof of Theorem 18. (1) Follows from Fact 5.

(2) By Lemma 3.6 in [13], every infinite compact Abelian group $X$ contains a dense characterized subgroup $H$. By Fact $1, H$ is $T$-characterized. Since every $G_{\delta}$-subgroup of $X$ is closed in $X$ by Proposition 2.4 of [13], $H$ is not a $G_{\delta}$-subgroup of $X$.

(3) Follows from Theorem 14 and the aforementioned Proposition 2.4 of [13].

(4) Follows from Fact 5.

(5) Follows from Corollary 17.

It is trivial that $\operatorname{Char}_{T}(X) \subseteq \operatorname{Char}(X)$ for every compact Abelian group $X$. For the circle group $\mathbb{T}$, we have:

Proposition 33. $\operatorname{Char}_{T}(\mathbb{T})=\operatorname{Char}(\mathbb{T})$.

Proof. We have to show only that $\operatorname{Char}(\mathbb{T}) \subseteq \operatorname{Char}_{T}(\mathbb{T})$. Let $H=s_{\mathbf{u}}(\mathbb{T}) \in \operatorname{Char}(\mathbb{T})$ for some sequence $\mathbf{u}$ in $\mathbb{Z}$.

If $H$ is infinite, then $H$ is dense in $\mathbb{T}$. Therefore, $\mathbf{u}$ is a $T$-sequence in $\mathbb{Z}$ by Fact 1. Thus, $H \in \operatorname{Char}_{T}(\mathbb{T})$.

If $H$ is finite, then $H$ is closed in $\mathbb{T}$. Clearly, $H^{\perp}$ has infinite exponent. Thus, $H \in \mathrm{Char}_{T}(\mathbb{T})$ by Theorem 10 .

Note that, if a compact Abelian group $X$ satisfies the equality $\operatorname{Char}_{T}(X)=$ Char $(X)$, then $X$ is connected by Fact 3 and Theorem 14. This fact and Proposition 33 justify the next problem:

Problem 34. Does there exists a connected compact Abelian group $X$, such that $\operatorname{Char}_{T}(X) \neq \operatorname{Char}(X)$ ? Is it true that $\operatorname{Char}_{T}(X)=\operatorname{Char}(X)$ if and only if $X$ is connected?

For a compact Abelian group $X$, the set of all subgroups of $X$ that are both $F_{\sigma \delta^{-}}$and $G_{\delta \sigma^{-}}$-subsets of $X$ we denote by $S \Delta_{3}^{0}(X)$. To complete the study of the Borel hierarchy of (T-)characterized subgroups of $X$, we have to answer the next question.

Problem 35. Describe compact Abelian groups $X$ of infinite exponent for which $\operatorname{Char}(X) \subseteq$ $\mathrm{S} \Delta_{3}^{0}(X)$. For which compact Abelian groups $X$ of infinite exponent there exists a $T$-characterized subgroup $H$ that does not belong to $\mathrm{S} \Delta_{3}^{0}(X)$ ? 


\section{4. $\mathfrak{g}_{T}$-Closed and $\mathfrak{g}_{T}$-Dense Subgroups of Compact Abelian Groups}

The following closure operator $\mathfrak{g}$ of the category of Abelian topological groups is defined in [11]. Let $X$ be an Abelian topological group and $H$ its arbitrary subgroup. The closure operator $\mathfrak{g}=\mathfrak{g}_{X}$ is defined as follows:

$$
\mathfrak{g}_{X}(H):=\bigcap_{\mathbf{u} \in \widehat{X}^{\mathbb{N}}}\left\{s_{\mathbf{u}}(X): H \leq s_{\mathbf{u}}(X)\right\},
$$

and we say that $H$ is $\mathfrak{g}$-closed if $H=\mathfrak{g}(H)$, and $H$ is $\mathfrak{g}$-dense if $\mathfrak{g}(H)=X$.

The set of all $T$-sequences in the dual group $\widehat{X}$ of a compact Abelian group $X$ we denote by $\mathcal{T}_{s}(\widehat{X})$. Clearly, $\mathcal{T}_{s}(\widehat{X}) \varsubsetneqq \widehat{X}^{\mathbb{N}}$. Let $H$ be a subgroup of $X$. In analogy to the closure operator $\mathfrak{g}, \mathfrak{g}$-closure and $\mathfrak{g}$-density, the operator $\mathfrak{g}_{T}$ is defined as follows:

$$
\mathfrak{g}_{T}(H):=\bigcap_{\mathbf{u} \in \mathcal{T}_{\mathcal{s}}(\widehat{X})}\left\{s_{\mathbf{u}}(X): H \leq s_{\mathbf{u}}(X)\right\},
$$

and we say that $H$ is $\mathfrak{g}_{T}$-closed if $H=\mathfrak{g}_{T}(H)$, and $H$ is $\mathfrak{g}_{T}$-dense if $\mathfrak{g}_{T}(H)=X$.

In this section, we study some properties of $\mathfrak{g}_{T}$-closed and $\mathfrak{g}_{T}$-dense subgroups of a compact Abelian group X. Note that every $\mathfrak{g}$-dense subgroup of $X$ is dense by Lemma 2.12 of [11], but for $\mathfrak{g}_{T}$-dense subgroups, the situation changes:

Proposition 36. Let X be a compact Abelian group.

(1) If $H$ is a $\mathfrak{g}_{T}$-dense subgroup of $X$, then the closure $\bar{H}$ of $H$ is an open subgroup of $X$.

(2) Every open subgroup of a compact Abelian group $X$ is $\mathfrak{g}_{T}$-dense.

Proof. (1) Suppose for a contradiction that $\bar{H}$ is not open in $X$. Then, $X / \bar{H}$ is an infinite compact group. By Lemma 3.6 of [13], $X / \bar{H}$ has a proper dense characterized subgroup $S$. Fact 1 implies that $S$ is a $T$-characterized subgroup of $X / \bar{H}$. Let $q: X \rightarrow$ $X / \bar{H}$ be the quotient map. Then, Lemma 24 yields that $q^{-1}(S)$ is a $T$-characterized dense subgroup of $X$ containing $H$. Since $q^{-1}(S) \neq X$, we obtain that $H$ is not $\mathfrak{g}_{T}$-dense in $X$, a contradiction.

(2) Let $H$ be an open subgroup of $X$. If $H=X$, the assertion is trivial. Assume that $H$ is a proper subgroup (so $X$ is disconnected). Let $\mathbf{u}$ be an arbitrary $T$-sequence, such that $H \subseteq s_{\mathbf{u}}(X)$. Since $H$ is open, $s_{\mathbf{u}}(X)$ is open, as well. Now, Corollary 13 implies that $s_{\mathbf{u}}(X)=X$. Thus, $H$ is $\mathfrak{g}_{T}$-dense in $X$.

Proposition 36(2) shows that $\mathfrak{g}_{T}$-density may essentially differ from the usual $\mathfrak{g}$-density. In the next theorem, we characterize all compact Abelian groups for which all $\mathfrak{g}_{T}$-dense subgroups are also dense. 
Theorem 37. All $\mathfrak{g}_{T}$-dense subgroups of a compact Abelian group $X$ are dense if and only if $X$ is connected.

Proof. Assume that all $\mathfrak{g}_{T}$-dense subgroup of $X$ are dense. Proposition 36(2) implies that $X$ has no open proper subgroups. Thus, $X$ is connected by [24] (7.9).

Conversely, let $X$ be connected and $H$ be a $\mathfrak{g}_{T}$-dense subgroup of $X$. Proposition 36(1) implies that the closure $\bar{H}$ of $H$ is an open subgroup of $X$. Since $X$ is connected, we obtain that $\bar{H}=X$. Thus, $H$ is dense in $X$.

For $\mathfrak{g}_{T}$-closed subgroups, we have:

Proposition 38. Let X be a compact Abelian group.

(1) Every proper open subgroup $H$ of $X$ is a $\mathfrak{g}$-closed non- $\mathfrak{g}_{T}$-closed subgroup.

(2) If every $\mathfrak{g}$-closed subgroup of $X$ is $\mathfrak{g}_{T}$-closed, then $X$ is connected.

Proof. (1) The subgroup $H$ is $\mathfrak{g}_{T}$-dense in $X$ by Proposition 36 . Therefore, $H$ is not $\mathfrak{g}_{T}$-closed. On the other hand, $H$ is $\mathfrak{g}$-closed in $X$ by Theorem A of [13].

(2) Item (1) implies that $X$ has no open subgroups. Thus, $X$ is connected by [24] (7.9).

We do not know whether the converse in Proposition 38(2) holds true:

Problem 39. Let a compact Abelian group X be connected. Is it true that every $\mathfrak{g}$-closed subgroup of $X$ is also $\mathfrak{g}_{T}$-closed?

Conflicts of Interest: The authors declare no conflict of interest.

\section{References}

1. Graev, M. Free topological groups. Izv. Akad. Nauk SSSR Ser. Mat. 1948, 12, 278-324.

2. Nienhuys, J. Construction of group topologies on Abelian groups. Fund. Math. 1972, 75, 101-116.

3. Protasov, I.V.; Zelenyuk, E.G. Topologies on abelian groups. Math. USSR Izv. 1991, 37, 445-460.

4. Raczkowski, S.U. Totally bounded topological group topologies on the integers. Topol. Appl. 2002, 121, 63-74.

5. Barbieri, G.; Dikranjan, D.; Milan, C.; Weber, H. Answer to Raczkowski's question on convergent sequences of integers. Topol. Appl. 2003, 132, 89-101.

6. Barbieri, G.; Dikranjan, D.; Milan, C.; Weber, H. Convergent sequences in precompact group topologies. Appl. Gen. Topol. 2005, 6, 149-169.

7. Gabriyelyan, S. Characterization of almost maximally almost-periodic groups. Topol. Appl. 2009, 156, 2214-2219.

8. Gabriyelyan, S. Finitely generated subgroups as a von Neumann radical of an Abelian group. Mat. Stud. 2012, 38, 124-138. 
9. Gabriyelyan, S. Bounded subgroups as a von Neumann radical of an Abelian group. Topol. Appl. 2014, 178, 185-199.

10. Protasov, I.V.; Zelenyuk, E.G. Topologies on Groups Determined by Sequences; Monograph Series; Math. Studies VNTL, L'viv, 1999.

11. Dikranjan, D.; Milan, C.; Tonolo, A. A characterization of the MAP abelian groups. J. Pure Appl. Algebra 2005, 197, 23-41.

12. Biró, A.; Deshouillers, J.-M.; Sós, V. Good approximation and characterization of subgroups of $\mathbb{R} / \mathbb{Z}$. Studia Sci. Math. Hungar. 2001, 38, 97-113.

13. Dikranjan, D.; Gabriyelyan, S. On characterized subgroups of compact abelian groups. Topol. Appl. 2013, 160, 2427-2442.

14. Beiglböck, M.; Steineder, C.; Winkler, R. Sequences and filters of characters characterizing subgroups of compact abelian groups. Topol. Appl. 2006, 153, 1682-1695.

15. Dikranjan, D.; Kunen, K. Characterizing subgroups of compact abelian groups. J. Pure Appl. Algebra 2007, 208, 285-291.

16. Gabriyelyan, S. On T-sequences and characterized subgroups. Topol. Appl. 2010, 157, 2834-2843.

17. Comfort, W.; Raczkowski, S.; Trigos-Arrieta, F.-J. Making group topologies with, and without, convergent sequences. Appl. Gen. Topol. 2006, 7, 109-124.

18. Gabriyelyan, S. On a generalization of Abelian sequential groups. Fund. Math. 2013, 221, 95-127.

19. Noble, N. $k$-groups and duality. Trans. Amer. Math. Soc. 1970, 151, 551-561.

20. Dikranjan, D.; Gabriyelyan, S.; Tarieladze, V. Characterizing sequences for precompact group topologies. J. Math. Anal. Appl. 2014, 412, 505-519.

21. Gabriyelyan, S. Characterizable groups: Some results and open questions. Topol. Appl. 2012, 159, 2378-2391.

22. Banaszczyk, W.; Chasco, M.; Martin-Peinador, E. Open subgroups and Pontryagin duality. Math. Z. 1994, 215, 195-204.

23. Außenhofer, L. Contributions to the Duality Theory of Abelian Topological Groups and to the Theory of Nuclear Groups. Diss. Math. 1999, 384, 113.

24. Hewitt, E.; Ross, K. Abstract Harmonic Analysis, 2nd ed.; Springer-Verlag: Berlin, Germany, 1979; Volume I.

25. Gabriyelyan, S. Groups of quasi-invariance and the Pontryagin duality. Topol. Appl. 2010, 157, 2786-2802.

26. Aaronson, J.; Nadkarni, M. $L_{\infty}$ eigenvalues and $L_{2}$ spectra of non-singular transformations. Proc. Lond. Math. Soc. 1987, 55, 538-570.

27. Gabriyelyan, S. Reflexive group topologies on Abelian groups. J. Group Theory 2010, 13, 891-901. 


\section{Characterized Subgroups of Topological Abelian Groups}

\section{Dikran Dikranjan, Anna Giordano Bruno and Daniele Impieri}

Abstract: A subgroup $H$ of a topological abelian group $X$ is said to be characterized by a sequence $\mathbf{v}=\left(v_{n}\right)$ of characters of $X$ if $H=\left\{x \in X: v_{n}(x) \rightarrow 0\right.$ in $\left.\mathbb{T}\right\}$. We study the basic properties of characterized subgroups in the general setting, extending results known in the compact case. For a better description, we isolate various types of characterized subgroups. Moreover, we introduce the relevant class of auto-characterized groups (namely, the groups that are characterized subgroups of themselves by means of a sequence of non-null characters); in the case of locally compact abelian groups, these are proven to be exactly the non-compact ones. As a by-product of our results, we find a complete description of the characterized subgroups of discrete abelian groups.

Reprinted from Axioms. Cite as: Dikranjan, D.; Bruno, A.G.; Impieri, D. Characterized Subgroups of Topological Abelian Groups. Axioms 2016, 4, 459-491.

\section{Introduction}

For a topological abelian group $X$, we denote by $\widehat{X}$ its dual group, that is the group of all characters of $X$ (i.e., continuous homomorphisms $X \rightarrow \mathbb{T}$ ). Following [1], for a sequence of characters $\mathbf{v}=\left(v_{n}\right) \in \widehat{X}^{\mathbb{N}}$, let:

$$
s_{\mathbf{v}}(X):=\left\{x \in X: v_{n}(x) \rightarrow 0\right\}
$$

which is always a subgroup of $X$. A subgroup $H$ of $X$ is said to be characterized if $H=s_{\mathbf{v}}(X)$ for some $\mathbf{v}=\left(v_{n}\right) \in \widehat{X}^{\mathbb{N}}$.

Historically, characterized subgroups were studied exclusively in the case of the circle group $\mathbb{T}=\mathbb{R} / \mathbb{Z}$ (see [2-5]), also in relation to Diophantine approximation, dynamical systems and ergodic theory (see [3,6,7]; one can find more on this topic in the nice survey [8], as well as in the more recent [9-12]). Some general results were then obtained in the case of metrizable compact abelian groups; for example, it is known that every countable subgroup of a metrizable compact abelian group is characterized (see [13, Theorem 1.4] and [14]), and it was pointed out in [14,15] that the metrizability is necessary, as a compact abelian group with a countable characterized subgroup is necessarily metrizable. Only recently, the case of general compact abelian groups was given full attention in [16], and a reduction theorem (to the metrizable case) was obtained. 
The few exceptions $[8,17,18]$ only confirm the tendency to study the characterized subgroups of $\mathbb{T}$ or, more recently, of compact abelian groups. To say the least, even the simplest case of characterized subgroups of discrete abelian groups has never been considered in the literature to the best of our knowledge.

The aim of these notes is to develop a general approach to characterized subgroups of arbitrary topological abelian groups, collecting the basic properties so far established in the compact case.

We isolate three special types of characterized subgroups, namely $T$-characterized, $\mathrm{K}$-characterized and $\mathrm{N}$-characterized subgroups (see Definition 3). Of those, T-characterized subgroups were introduced by Gabriyelyan in [11], K-characterized subgroups were substantially studied by Kunen and his coauthors in $[13,19,20]$, while $\mathrm{N}$-characterized subgroups, even if never introduced explicitly, have been frequently used in the theory of duality in topological abelian groups (being nothing else but the annihilators of countable sets of the dual group). One of the advantages of this articulation is the possibility to establish some general permanence properties that fail to be true in the whole class of characterized subgroups, but hold true in some of these subclasses. Moreover, we see that each characterized subgroup is either $\mathrm{N}$-characterized or coincides with the intersection of an $\mathrm{N}$-characterized subgroup and a K-characterized subgroup (see Corollary 3).

Inspired by the notion of $T$-characterized subgroup, we introduce also the stronger one of $T B$-characterized subgroup (see Definition 4). The following implications hold, and none of them can be reversed in general (see Section 5):

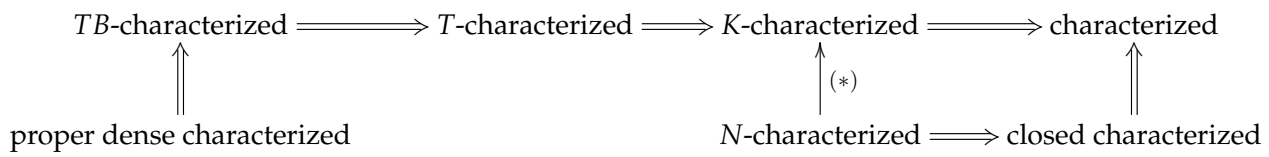

where $(*)$ holds under the assumption that the subgroup is closed and has infinite index (see Corollary 6).

In Section 6, we introduce the prominent class of auto-characterized groups (see Definition 5). These are the topological abelian groups that are characterized subgroups of themselves by means of a non-trivial sequence of characters (see (2)). The fact that compact abelian groups are not auto-characterized is equivalent to the well-known non-trivial fact that the Bohr topology of an infinite discrete abelian group has no non-trivial convergent sequences. Here, we generalize this fact by proving that the property of being non-auto-characterized describes the compact abelian groups within the class of all locally compact abelian groups (see Theorem 3 ). Moreover, in the general case, we describe the auto-characterized groups in terms of their Bohr compactification (see Theorem 5). 
We study the basic properties of $K$ - and of $N$-characterized subgroups respectively in Sections 7 and 8 . For the case of discrete abelian groups, which is considered here for the first time, we give a complete description of characterized subgroups by showing that these are precisely the subgroups of index at most $\mathfrak{c}$ and that a subgroup is characterized precisely when it is $K$ - and $N$-characterized (see Corollary 16).

In Section 7, we describe when a closed subgroup of infinite index is both $K$ - and $N$-characterized, and we see that this occurs precisely when it is only $N$-characterized (see Theorem 6); then, we consider the special case of open subgroups, proving that proper open subgroups of infinite index (respectively, of finite index) are $\mathrm{K}$-characterized if and only if they are characterized (respectively, auto-characterized) (see Theorems 7 and 8). In particular, no proper open subgroup of a compact abelian group is $K$-characterized.

In Section 8, extending a criterion for compact abelian groups given in [16], we show that for locally compact abelian groups one can reduce the study of characterized subgroups to the metrizable case (see Theorem 11). Moreover, we describe the closed characterized subgroups of the locally compact abelian groups by showing that they are precisely the $N$-characterized subgroups (see Theorem 12). As a consequence, we add other equivalent conditions to the known fact from [16] that a closed subgroup of a compact abelian group is characterized if and only if it is $G_{\delta}$, namely that the subgroup is $K$ - and $N$-characterized (see Theorem 13).

Section 9 concerns $T$-characterized subgroups of compact abelian groups. We establish a criterion to determine when a characterized subgroup of a compact abelian group is not $T$-characterized (see Theorem 15), which extends results from [11]. The impact on characterized subgroups of connected compact abelian groups is discussed.

The final Section 10 contains various comments and open problems, both general and specific.

\subsection{Notation and Terminology}

The symbol $\mathfrak{c}$ is used to denote the cardinality of continuum. The symbols $\mathbb{Z}, \mathbb{P}$, $\mathbb{N}$ and $\mathbb{N}_{+}$are used for the set of integers, the set of primes, the set of non-negative integers and the set of positive integers, respectively. The circle group $\mathbb{T}$ is identified with the quotient group $\mathbb{R} / \mathbb{Z}$ of the reals $\mathbb{R}$ and carries its usual compact topology. Let $\pi: \mathbb{R} \rightarrow \mathbb{T}$ be the canonical projection; the usual group norm $\|-\|$ on $\mathbb{T}$ is defined by $\|\pi(x)\|=d(x, \mathbb{Z})$ for every $x \in \mathbb{R}$. We denote by $\mathbb{T}_{+}$the image of $[-1 / 4,1 / 4]$ in $\mathbb{T}$. If $m$ is a positive integer, $G[m]=\{x \in G: m x=0\}$, and $\mathbb{Z}(m)$ is the cyclic group of order $m$. Moreover, for $p \in \mathbb{P}$, we denote by $\mathbb{Z}\left(p^{\infty}\right)$ and $\mathbb{J}_{p}$, respectively, the Prüfer group and the $p$-adic integers.

We say that an abelian group $G$ is torsion if every element of $G$ is torsion (i.e., for every $x \in G$, there exists $m \in \mathbb{N}_{+}$, such that $m x=0$ ). If $M$ is a subset of $G$, then $\langle M\rangle$ 
is the smallest subgroup of $G$ containing $M$. We denote by $\operatorname{Hom}(G, \mathbb{T})$ the group of the homomorphisms $G \rightarrow \mathbb{T}$.

For a topological space $X=(X, \tau)$, the weight $w(X)$ of $X$ is the minimum cardinality of a base for $\tau$. For a subset $A$ of $X$, we denote by $\bar{A}^{\tau}$ the closure of $A$ in $(X, \tau)$ (we write only $\bar{A}$ when there is no possibility of confusion).

A topological abelian group $X$ is totally bounded if for every open subset $U$ of 0 in $X$, there exists a finite subset $F$ of $X$, such that $U+F=X$. If $X$ is totally bounded and Hausdorff, we say that $X$ is precompact. We denote by $\widetilde{X}$ the two-sided completion of $X$; in case $X$ is precompact, $\widetilde{X}$ coincides with the Weil completion.

For a subset $A$ of $X$, the annihilator of $A$ in $\widehat{X}$ is $A^{\perp}=\{\chi \in \widehat{X}: \chi(A)=\{0\}\}$, and for a subset $B$ of $\widehat{X}$, the annihilator of $B$ in $X$ is $B^{\perp}=\{x \in X: \chi(x)=0$ for every $\chi \in B\}$.

We say that a sequence $\mathbf{v} \in \widehat{X}^{\mathbb{N}}$ is trivially null if there exists $n_{0} \in \mathbb{N}$, such that $v_{n}=0$ for every $n \geq n_{0}$, and we say that $\mathbf{v}$ is non-trivial if it is not trivially null.

\section{Background on Topological Groups}

\subsection{Basic Definitions}

Let $G$ be an abelian group and $H$ a subgroup of $\operatorname{Hom}(G, \mathbb{T})$. Let $T_{H}$ be the weakest group topology on $G$, such that all elements of $H$ are continuous with respect to $T_{H}$; then $T_{H}$ is totally bounded. The other way around, Comfort and Ross proved that any totally bounded group topology is of this type (see [21, Theorem 1.2]).

Theorem 1. [21, Theorems 1.2, 1.3 and 1.11, Corollary 1.4] Let $G$ be an abelian group and $H$ a subgroup of $\operatorname{Hom}(G, \mathbb{T})$. Then, $T_{H}$ is totally bounded and:

(a) $T_{H}$ is Hausdorff if and only if $H$ separates the points of $G$;

(b) $T_{H}$ is metrizable if and only if $H$ is countable.

The following two notions will be often used in the paper.

Definition 1. A topological abelian group $X$ is said to be:

(i) maximally almost periodic $(M A P)$ if $\widehat{X}$ separates the points of $X$;

(ii) minimally almost periodic (MinAP) if $\widehat{X}=\{0\}$.

We recall that two group topologies $\tau_{1}$ and $\tau_{2}$ on an abelian group $X$ are compatible if they have the same characters, that is $\widehat{\left(X, \tau_{1}\right)}=\widehat{\left(X, \tau_{2}\right)}$.

If $X=(X, \tau)$ is a topological abelian group, denote by $\tau^{+}$its Bohr topology, that is the finest totally bounded group topology on $X$ coarser than $\tau$ (indeed, $\tau^{+}=T_{\widehat{X}}$ ); we denote $X$ endowed with its Bohr topology also by $X^{+}$, and we call $\tau^{+}$also the Bohr modification of $\tau$. Clearly, $\tau$ and $\tau^{+}$are compatible. Moreover, 
(i) $\tau$ is MAP if and only if $\tau^{+}$is Hausdorff;

(ii) $\tau$ is MinAP if and only if $\tau^{+}$is indiscrete.

A subgroup $H$ of $(X, \tau)$ is:

(a) dually closed if $H$ is $\tau^{+}$-closed (or, equivalently, $X / H$ is MAP);

(b) dually embedded if every $\chi \in \widehat{H}$ can be extended to $X$.

Clearly, dually closed implies closed, since $H \leq \bar{H}^{\tau} \leq \bar{H}^{\tau^{+}}$.

Fact 1 . Let $X$ be a locally compact abelian group. Then:

(i) every closed subgroup $H$ of $X$ is dually closed, i.e., $X / H$ is $M A P$;

(ii) in particular, every locally compact abelian group is MAP;

(iii) $X$ and $X^{+}$have the same closed subgroups;

(iv) consequently, $X$ is separable if and only if $X^{+}$is separable.

For a topological abelian group $X$ and a subgroup $L$ of $X$, the weak topology $\sigma(\widehat{X}, L)$ of the dual $\widehat{X}$ is the totally bounded group topology of $\widehat{X}$ generated by the elements of $L$ considered as characters of $\widehat{X}$; namely, for every $x \in L$, consider $\xi_{x}: \widehat{X} \rightarrow \mathbb{T}$ defined by $\xi_{x}(\chi)=\chi(x)$ for every $\chi \in \widehat{X}$. A local base of $\sigma(\widehat{X}, L)$ is given by the finite intersections of the sets $\xi_{x}^{-1}(U)$, where $x \in L$ and $U$ is an open neighborhood of 0 in $\mathbb{T}$. Clearly, if $L_{1} \leq L_{2}$, then $\sigma\left(\widehat{X}, L_{1}\right) \leq \sigma\left(\widehat{X}, L_{2}\right)$.

Note that the weak topology $\sigma(\widehat{X}, X)$ is coarser than the compact-open topology on $\hat{X}$. If $L$ separates the points of $\widehat{X}$ (e.g., when $L$ is dense in $X$ or when $L=X$ ), then $\sigma(\widehat{X}, L)$ is precompact.

Fact 2. If $X$ is a reflexive topological abelian group, then $\sigma(\widehat{X}, X)$ coincides with the Bohr topology of $\widehat{X}$.

We recall that a sequence $\mathbf{v}$ in an abelian group $G$ is a $T$-sequence (respectively, $T B$-sequence) if there exists a Hausdorff (respectively, precompact) group topology $\tau$ on $G$, such that $\mathbf{v}$ is a null sequence in $(G, \tau)$.

Lemma 1. Let $X$ be a topological abelian group and $\mathbf{v} \in \widehat{X}^{\mathbb{N}}$. Then:

(i) for a subgroup $L$ of $X, v_{n}(x) \rightarrow 0$ in $\mathbb{T}$ for every $x \in L$ if and only if $v_{n} \rightarrow 0$ in $\sigma(\widehat{X}, L)$;

(ii) if $s_{\mathbf{v}}(X)$ is dense in $X$, then $\mathbf{v}$ is a TB-sequence.

Proof. (i) follows from the definition of $\sigma(\widehat{X}, L)$.

(ii) As $s_{\mathbf{v}}(X)$ is dense in $X$, then $\sigma\left(\widehat{X}, s_{\mathbf{v}}(X)\right)$ is precompact. By item (i), $v_{n} \rightarrow 0$ in $\sigma\left(\widehat{X}, s_{\mathbf{v}}(X)\right)$; hence, $\mathbf{v}$ is a $T B$-sequence. 
Let $G$ be a discrete abelian group. For a sequence $\mathbf{v} \in G^{\mathbb{N}}$, the group topology:

$$
\sigma_{\mathbf{v}}:=T_{S_{\mathbf{v}}(\widehat{G})}
$$

is the finest totally bounded group topology on $G$, such that $\mathbf{v}$ is a null sequence in $\left(G, \sigma_{\mathbf{v}}\right)$.

Fact 3. [1, Lemma 3.1, Proposition 3.2] Let $G$ be a discrete abelian group and $\mathbf{v} \in G^{\mathbb{N}}$. The following conditions are equivalent:

(i) $\mathbf{v}$ is a TB-sequence;

(ii) $\sigma_{\mathbf{v}}$ is Hausdorff;

(ii) $s_{\mathbf{v}}(\widehat{G})$ is dense in $\widehat{G}$.

\subsection{Useful Folklore Results}

We recall the following basic properties that will be used in the paper. Although most of them are well known, we offer proofs for the reader's convenience.

Lemma 2. Let $X$ be a topological abelian group and $H$ a subgroup of $X$. Then:

(i) $\widehat{X / H}$ is algebraically isomorphic to $H^{\perp}$;

(ii) $\widehat{X} / H^{\perp}$ is algebraically isomorphic to a subgroup of $\widehat{H}$.

Proof. (i) Let $\psi: \widehat{X / H} \rightarrow \widehat{X}$ be defined by $\chi \mapsto \chi \circ \pi$, where $\pi: X \rightarrow X / H$ is the canonical projection. Then, $\psi$ is injective, and its image is $H^{\perp}$.

(ii) Let $\rho: \widehat{X} \rightarrow \widehat{H}$ be defined by $\chi \mapsto \chi \uparrow_{H}$. Then, $\operatorname{ker} \rho=H^{\perp}$, and so, we get the thesis.

The following fact follows from the equivalence of items (a) and (e) of [4, Exercise 3.8.25]. Since no proofs are given there, we offer a proof for the reader's convenience.

Lemma 3. A compact abelian group $K$ is separable if and only if $w(K) \leq \mathfrak{c}$.

Proof. The inequality $w(K) \leq c$ holds for every separable regular topological space $K$.

Assume that $w(K) \leq c$. The discrete abelian group $X=\widehat{K}$ has size $|X|=$ $w(K) \leq c$. Consider the embedding $i: X \rightarrow D(X)$, where $D(X)$ is the divisible hull of $X$. Then, $|D(X)| \leq \mathfrak{c}$ and $D(X)=\bigoplus_{i \in I} D_{i}$, for some countable divisible abelian groups $D_{i}$ and a set of indices $I$ with $|I| \leq \mathfrak{c}$. Therefore, $\widehat{i}: \prod_{i \in I} \widehat{D}_{i} \rightarrow \widehat{X}=K$ is a surjective continuous homomorphism, and each $\widehat{D}_{i}$ is a metrizable compact abelian group. By the Hewitt-Marczewski-Pondiczery Theorem, since $|I| \leq \mathfrak{c}$, we have that $\prod_{i \in I} \widehat{D}_{i}$ is separable; hence, $K$ is separable, as well. 
Lemma 4. Let $X$ be a precompact abelian group. Then, the singleton $\{0\}$ is $G_{\delta}$ if and only if there exists a continuous injection $X \rightarrow \mathbb{T}^{\mathbb{N}}$.

Proof. If there exists a continuous injection $X \rightarrow \mathbb{T}^{\mathbb{N}}$, then $\{0\}$ is $G_{\delta}$ in $X$, as it is $G_{\delta}$ in $\mathbb{T}^{\mathbb{N}}$.

Assume now that $\{0\}=\bigcap_{n \in \mathbb{N}} U_{n}$, where each $U_{n}$ is an open subset of $X$, and we can assume that $U_{n}$ is in the prebase of the neighborhoods of 0 in $X$. Therefore, for every $n \in \mathbb{N}$, there exist $v_{n} \in \widehat{X}$ and an open neighborhood $V_{n}$ of 0 in $\mathbb{T}$ containing no non-trivial subgroup of $\mathbb{T}$, such that $U_{n}=v_{n}^{-1}\left(V_{n}\right)$. Then, $\{0\}=\bigcap_{n \in \mathbb{N}} \operatorname{ker} v_{n}$. Hence, $j: X \rightarrow \mathbb{T}^{\mathbb{N}}$ defined by $j(x)=\left(v_{n}(x)\right)_{n \in \mathbb{N}}$ is a continuous injective homomorphism.

Theorem 2. Let $X$ be a locally compact abelian group. Then, $X$ is metrizable with $|X| \leq \mathfrak{c}$ if and only if there exists a continuous injective homomorphism $X \rightarrow \mathbb{T}^{\mathbb{N}}$.

Proof. If there exists a continuous injective homomorphism $X \rightarrow \mathbb{T}^{\mathbb{N}}$, then clearly $X$ is metrizable and $|X| \leq\left|\mathbb{T}^{\mathbb{N}}\right|=\mathfrak{c}$.

Suppose now that $X$ is metrizable and has cardinality at most $\mathfrak{c}$. It is well known (for example, see [22]) that $X=\mathbb{R}^{n} \times X_{0}$, where $n \in \mathbb{N}$ and $X_{0}$ is a locally compact abelian group admitting an open compact (metrizable) subgroup $K$. Clearly, there exist two continuous injective homomorphisms $j_{1}: \mathbb{R}^{n} \hookrightarrow \mathbb{T}^{\mathbb{N}}$ and $j_{2}: K \hookrightarrow$ $\mathbb{T}^{\mathbb{N}}$. Therefore, $j_{3}=\left(j_{1}, j_{2}\right): \mathbb{R}^{n} \times K \rightarrow \mathbb{T}^{\mathbb{N}} \times \mathbb{T}^{\mathbb{N}} \cong \mathbb{T}^{\mathbb{N}}$ is an injective continuous homomorphism, too. Since $\mathbb{T}^{\mathbb{N}}$ is divisible and $\mathbb{R}^{n} \times K$ is open in $X, j_{3}$ extends continuously to $\widetilde{j_{3}}: X \rightarrow \mathbb{T}^{\mathbb{N}}$. Let $\pi: X \rightarrow X /\left(\mathbb{R}^{n} \times K\right)$ be the canonical projection. Since $X /\left(\mathbb{R}^{n} \times K\right)$ is discrete, there exists a continuous injective homomorphism $j_{4}: X /\left(\mathbb{R}^{n} \times K\right) \rightarrow \mathbb{T}^{\mathbb{N}}$. Let $\varphi=j_{4} \circ \pi: X \rightarrow \mathbb{T}^{\mathbb{N}}$.

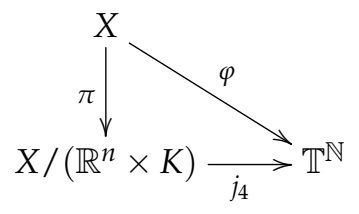

Let now $j: X \rightarrow \mathbb{T}^{\mathbb{N}} \times \mathbb{T}^{\mathbb{N}} \cong \mathbb{T}^{\mathbb{N}}$ be defined by $j(x)=\left(\varphi(x), \widetilde{j_{3}}(x)\right)$ for every $x \in X$. Then, $j$ is continuous, since $\varphi$ and $\widetilde{j_{3}}$ are continuous. Moreover, $j$ is injective, as $j(x)=0$ for some $x \in X$ implies $\varphi(x)=0$ and $\widetilde{j_{3}}(x)=0$; therefore, $x \in \mathbb{R}^{n} \times K$, and so, since $\widetilde{j_{3}}\left\lceil_{\mathbb{R}^{n} \times K}=j_{3}\right.$ is injective, one has $x=0$.

\section{General Permanence Properties of Characterized Subgroups}

Let $X$ be a topological abelian group, and denote by $\mathfrak{C h a r}(X)$ the family of all subgroups of $X$ that are characterized. 
We start by observing that:

$$
\text { if } \mathbf{v} \in \widehat{X}^{\mathbb{N}} \text { is trivially null, then } X=s_{\mathbf{v}}(X)
$$

The following are basic facts on characterized subgroups (see $[1,13,16,23])$; we give a proof for the reader's convenience.

Lemma 5. Let $X$ be a topological abelian group and $\mathbf{v} \in \widehat{X}^{\mathbb{N}}$. Then:

(i) for every subgroup J of $X, s_{\mathbf{v}^{*}}(J)=s_{\mathbf{v}}(X) \cap J$, where $v_{n}^{*}=v_{n} \uparrow_{J}$ for every $n \in \mathbb{N}$;

(ii) $s_{\mathbf{v}}(X)=s_{\mathbf{u}}(X)$ if $\mathbf{u}$ is any permutation of $\mathbf{v}$;

(iii) $\mathfrak{C h a r}(X)$ is stable under taking finite intersections;

(iv) $s_{\mathbf{v}}(X)$ is an $F_{\sigma \delta}$-set (i.e., countable intersection of countable unions of closed subsets).

Proof. Items (i) and (ii) are obvious. To prove (iii), if $\mathbf{u}, \mathbf{v} \in \widehat{X}^{\mathbb{N}}$, define $\mathbf{w}=\left(w_{n}\right)$, where $w_{2 n}=u_{n}$ and $w_{2 n+1}=v_{n}$ for every $n \in \mathbb{N}$; hence, $s_{\mathbf{u}}(X) \cap s_{\mathbf{v}}(X)=$ $s_{\mathbf{w}}(X)$. To prove (iv), note that $s_{\mathbf{v}}(X)=\bigcap_{m} \bigcup_{k} \bigcap_{n \geq k} S_{n, m}$, where each $S_{n, m}=$ $\left\{x \in X:\left\|v_{n}(x)\right\| \leq \frac{1}{m}\right\}$ is a closed subset of $X$.

Now, we prove that, under suitable hypotheses, the relation of being a characterized subgroup is transitive:

Proposition 1. Let $X$ be a topological abelian group and $X_{0}, X_{1}, X_{2}$ subgroups of $X$ with $X_{0} \leq X_{1} \leq X_{2}$ and such that $X_{1}$ is dually embedded in $X_{2}$. If $X_{0} \in \mathfrak{C h a r}\left(X_{1}\right)$ and $X_{1} \in \mathfrak{C h a r}\left(X_{2}\right)$, then $X_{0} \in \mathfrak{C h a r}\left(X_{2}\right)$.

Proof. Let $\mathbf{v} \in \widehat{X}_{1}^{\mathbb{N}}$, such that $X_{0}=s_{\mathbf{v}}\left(X_{1}\right)$, and let $\mathbf{w} \in \widehat{X}_{2}^{\mathbb{N}}$, such that $X_{1}=s_{\mathbf{w}}\left(X_{2}\right)$. As $X_{1}$ is dually embedded in $X_{2}, v_{n}$ extends to a character $v_{n}^{*}$ of $X_{2}$ for every $n \in \mathbb{N}$; so, let $\mathbf{v}^{*}=\left(v_{n}^{*}\right) \in \widehat{X}_{2}^{\mathbb{N}}$. Define $\mathbf{w}^{*} \in \widehat{X}_{2}^{\mathbb{N}}$ by letting $w_{2 n}^{*}=v_{n}^{*}$ and $w_{2 n+1}^{*}=w_{n}$ for every $n \in \mathbb{N}$. Then, by Lemma $5(\mathrm{i})$,

$$
s_{\mathbf{w}^{*}}\left(X_{2}\right)=s_{\mathbf{v}^{*}}\left(X_{2}\right) \cap s_{\mathbf{w}}\left(X_{2}\right)=s_{\mathbf{v}^{*}}\left(X_{2}\right) \cap X_{1}=s_{\mathbf{v}}\left(X_{1}\right)=X_{0},
$$

so $X_{0} \in \mathfrak{C h a r}\left(X_{2}\right)$, as required.

Clearly, two compatible group topologies have the same characterized subgroups:

Lemma 6. If $\tau_{1}$ and $\tau_{2}$ are compatible group topologies on an abelian group $X$, then $\mathfrak{C h a r}\left(X, \tau_{1}\right)=\mathfrak{C h a r}\left(X, \tau_{2}\right)$.

In particular, for a topological abelian group $(X, \tau)$, since $\tau$ and its Bohr modification $\tau^{+}$are compatible, $\mathfrak{C h a r}(X, \tau)=\mathfrak{C h a r}\left(X, \tau^{+}\right)$. 


\section{The $\Gamma$-Radical}

Definition 2. Let $X$ be a topological abelian group. For a subset $\Gamma$ of $\widehat{X}$, define the $\Gamma$-radical of $X$ by:

$$
n_{\Gamma}(X):=\bigcap_{\chi \in \Gamma} \operatorname{ker} \chi=\Gamma^{\perp} .
$$

Clearly, $n_{\Gamma}(X)$ is a closed subgroup of $X$.

The motivation for the choice of the term $\Gamma$-radical is the special case $\Gamma=\widehat{X}$, when

$$
n(X):=n_{\widehat{X}}(X)
$$

is usually called the von Neumann radical of $X$. Then, $n(X)=\{0\}$ (respectively, $n(X)=X$ ) precisely when $\widehat{X}$ separates the points of $X$ (respectively, $\widehat{X}=\{0\}$ ); in other words:

(i) $X$ is MAP if and only if $n(X)=\{0\}$;

(ii) $X$ is MinAP if and only if $n(X)=X$.

Remark 1. Let $X$ be a topological abelian group and $\Gamma$ a subset of $\widehat{X}$.

(i) If $\Gamma=\varnothing$, then $n_{\Gamma}(X)=X$.

(ii) If $\Gamma$ is countable, then $n_{\Gamma}(X)$ is a characterized subgroup of $X$ (indeed, $n_{\Gamma}(X)=s_{\mathbf{v}}(X)$ for $\mathbf{v} \in \widehat{X}^{\mathbb{N}}$, such that each character in $\Gamma$ appears infinitely many times in $\mathbf{v})$.

For a given sequence $\mathbf{v} \in \widehat{X}^{\mathbb{N}}$, the support $\Gamma_{\mathbf{v}}=\left\{v_{n}: n \in \mathbb{N}\right\}$ of $\mathbf{v}$ is the set of all characters appearing in $\mathbf{v}$. We abbreviate the notation of the $\Gamma_{\mathbf{v}}$-radical by writing:

$$
n_{\mathbf{v}}(X):=n_{\Gamma_{\mathbf{v}}}(X)
$$

and we call this subgroup the v-radical of $X$.

Lemma 7. Let $X$ be a topological abelian group and $\mathbf{v} \in \widehat{X}^{\mathbb{N}}$. Then:

(i) $n_{\mathbf{v}}(X) \leq s_{\mathbf{v}}(X)$;

(ii) $n_{\mathbf{v}}(X)$ is dually closed;

(iii) $n_{\mathbf{v}}(X)$ is characterized;

(iv) $n_{\mathbf{V}}(X)$ is closed, and $\{0\}$ is $G_{\delta}$ in $X / n_{\mathbf{v}}(X)\left(\right.$ so, $n_{\mathbf{v}}(X)$ is $\left.G_{\delta}\right)$;

(v) $\left[X: n_{\mathbf{v}}(X)\right] \leq \mathfrak{c}$.

Proof. (i) and (ii) are clear from the definitions, and (iii) follows from Remark 1(ii).

(iv) Let $\varphi: X \rightarrow \mathbb{T}^{\mathbb{N}}$ be defined by $\varphi(x)=\left(v_{0}(x), \ldots, v_{n}(x), \ldots\right)$ for every $x \in X$. Since $\{0\}$ is $G_{\delta}$ in $\mathbb{T}^{\mathbb{N}}$, we conclude that $\{0\}$ is $G_{\delta}$ in $X / n_{\mathbf{v}}(X)$. Moreover, $\operatorname{ker} \varphi=\varphi^{-1}(0)=n_{\mathbf{v}}(X)$, so $n_{\mathbf{v}}(X)$ is $G_{\delta}$ in $X$. 
(v) Since $X / n_{\mathbf{v}}(X)$ is algebraically isomorphic to $\varphi(X) \leq \mathbb{T}^{\mathbb{N}}$ and $\left|\mathbb{T}^{\mathbb{N}}\right|=\mathfrak{c}$, we conclude that $\left[X: n_{\mathbf{v}}(X)\right] \leq \mathfrak{c}$.

Remark 2. Let $X$ be a topological abelian group and $\mathbf{v} \in \widehat{X}^{\mathbb{N}}$. Then, $n_{\mathbf{v}}(X)$ is closed and $G_{\delta}$ in every group topology on $X$ that makes $v_{n}$ continuous for every $n \in \mathbb{N}$. In particular, $n_{\mathbf{v}}(X)$ is closed and $G_{\delta}$ in every group topology on $X$ compatible with the topology of $X$, so in the Bohr topology of $X$.

Lemma 7 gives a bound for the index of the characterized subgroups:

Corollary 1. Every characterized subgroup of a topological abelian group X has index at most $c$.

Proof. Let $\mathbf{v} \in \widehat{X}^{\mathbb{N}}$. Since $n_{\mathbf{v}}(X) \leq s_{\mathbf{v}}(X)$ by Lemma $7(i)$; hence, $\left[X: s_{\mathbf{v}}(X)\right] \leq[X$ : $\left.n_{\mathbf{v}}(X)\right] \leq \mathfrak{c}$ by Lemma $7(\mathrm{v})$.

The set $\Gamma_{\mathbf{v}}$ can be partitioned as

$$
\Gamma_{\mathbf{v}}=\Gamma_{\mathbf{v}}^{\infty} \dot{\cup} \Gamma_{\mathbf{v}}^{0}
$$

where:

(i) $\Gamma_{\mathbf{v}}^{\infty}:=\left\{v_{n} \in \Gamma_{\mathbf{v}}: v_{n}=v_{m}\right.$ for infinitely many $\left.m \in \mathbb{N}\right\}$;

(ii) $\Gamma_{\mathbf{v}}^{0}:=\Gamma_{\mathbf{v}} \backslash \Gamma_{\mathbf{v}}^{\infty}$.

In other words, $\Gamma_{\mathbf{v}}^{\infty}$ is the set of all characters appearing infinitely many times in $\mathbf{v}$, while each character in its complement $\Gamma_{\mathbf{v}}^{0}$ appears finitely many times in $\mathbf{v}$. Clearly, $\mathbf{v}$ is a finitely many-to-one sequence if and only if $\Gamma_{\mathbf{v}}^{\infty}=\varnothing$.

In case $\Gamma_{\mathbf{v}}^{\infty} \neq \varnothing$, let $\mathbf{v}^{\infty}$ be the largest subsequence of $\mathbf{v}$ with $\Gamma_{\mathbf{v}^{\infty}}=\Gamma_{\mathbf{v}}^{\infty}$. Then, clearly, $s_{\mathbf{v}^{\infty}}(X)=n_{\mathbf{v}^{\infty}}(X)$.

In case $\Gamma_{\mathbf{v}}^{0}$ is finite, the subsequence $\mathbf{v}^{\infty}$ of $\mathbf{v}$ is cofinite, so $s_{\mathbf{v}}(X)=s_{\mathbf{v}^{\infty}}(X)$. In other words, one can safely replace $\mathbf{v}$ by $\mathbf{v}^{\infty}$. This is why from now on, we shall always assume that:

$$
\text { either } \Gamma_{\mathbf{v}}^{0}=\varnothing \text { or } \Gamma_{\mathbf{v}}^{0} \text { is infinite. }
$$

If $\Gamma_{\mathbf{v}}^{0}$ is infinite, we denote by $\mathbf{v}^{0}$ the subsequence of $\mathbf{v}$ such that $\Gamma_{\mathbf{v}^{0}}=\Gamma_{\mathbf{v}}^{0}$. If $\Gamma_{\mathbf{v}}^{\infty} \neq \varnothing$, we have the partition

$$
\mathbf{v}=\mathbf{v}^{\infty} \dot{\cup} \mathbf{v}^{0}
$$

of $\mathbf{v}$ in the two subsequences $\mathbf{v}^{\infty}$ and $\mathbf{v}^{0}$. Moreover, always $\Gamma_{\mathbf{v}^{0}}^{\infty}=\varnothing$, and so, if $\Gamma_{\mathbf{v}^{0}}$ is infinite (equivalently, $\Gamma_{\mathbf{v}}^{0} \neq \varnothing$ by (3)), $\mathbf{v}^{0}$ is a finitely many-to-one sequence and $s_{\mathbf{v}^{0}}(X)=s_{\mathbf{w}}(X)$, where $\mathbf{w}$ is a one-to-one subsequence of $\mathbf{v}^{0}$ such that $\Gamma_{\mathbf{w}}=\Gamma_{\mathbf{v}^{0}}=\Gamma_{\mathbf{v}}^{0}$. 
We see now how we can obtain the subgroup of $X$ characterized by $\mathbf{v}$ by considering separately the $\mathbf{v}^{\infty}$-radical of $X$ and the subgroup of $X$ characterized by $\mathbf{v}^{0}$.

Lemma 8. Let $X$ be a topological abelian group and $\mathbf{v} \in \widehat{X}^{\mathbb{N}}$ satisfying (3).

(i) If $\Gamma_{\mathbf{v}}^{0}=\varnothing$, then $\mathbf{v}^{\infty}=\mathbf{v}$, so $\Gamma_{\mathbf{v}}^{\infty} \neq \varnothing$ and $s_{\mathbf{v}}(X)=n_{\mathbf{v}^{\infty}}(X)$.

(ii) If $\Gamma_{\mathbf{v}}^{0}$ is infinite and $\Gamma_{\mathbf{v}}^{\infty} \neq \varnothing$, then $s_{\mathbf{v}}(X)=s_{\mathbf{v}^{0}}(X) \cap n_{\mathbf{v}^{\infty}}(X)$.

Proof. (i) Since $\Gamma_{\mathbf{v}}^{0}=\varnothing$, we have $s_{\mathbf{v}}(X)=s_{\mathbf{v}^{\infty}}(X)$, and as observed above, $s_{\mathbf{v}^{\infty}}(X)=n_{\mathbf{v}^{\infty}}(X)$.

(ii) Since $\mathbf{v}^{\infty}$ and $\mathbf{v}^{0}$ are subsequences of $\mathbf{v}$, it follows that $s_{\mathbf{v}}(X) \leq s_{\mathbf{v}^{0}}(X) \cap$ $n_{\mathbf{v}^{\infty}}(X)$. Let now $x \in s_{\mathbf{v}^{0}}(X) \cap n_{\mathbf{v}^{\infty}}(X)$. Since both $\mathbf{v}^{\infty}(x) \rightarrow 0$ and $\mathbf{v}^{0}(x) \rightarrow 0$ and since $\mathbf{v}=\mathbf{v}^{\infty} \cup \mathbf{v}^{0}$, we conclude that $\mathbf{v}(x) \rightarrow 0$, that is $x \in s_{\mathbf{v}}(X)$. This concludes the proof.

For $\mathbf{v}=\left(v_{n}\right) \in \widehat{X}^{\mathbb{N}}$ and $m \in \mathbb{N}$, let

$$
\mathbf{v}_{(m)}:=\left(v_{n}\right)_{n \geq m}
$$

Note that $n_{\mathbf{v}_{(m)}}(X) \leq n_{\mathbf{v}_{(m+1)}}(X)$ for every $m \in \mathbb{N}$.

\section{A Hierarchy for Characterized Subgroups}

The following definition introduces three specific types of characterized subgroups.

Definition 3. Let $X$ be a topological abelian group. A subgroup $H$ of $X$ is:

(i) T-characterized if $H=s_{\mathbf{v}}(X)$ where $\mathbf{v} \in \widehat{X}^{\mathbb{N}}$ is a non-trivial T-sequence;

(ii) K-characterized if $H=s_{\mathbf{v}}(X)$ for some finitely many-to-one sequence $\mathbf{v} \in \widehat{X}^{\mathbb{N}}$ (i.e., $\left.\Gamma_{\mathbf{v}}^{\infty}=\varnothing\right)$;

(iii) $N$-characterized if $H=n_{\mathbf{v}}(X)$ for some $\mathbf{v} \in \widehat{X}^{\mathbb{N}}$.

In analogy to Definition 3(i), we introduce the following smaller class of characterized subgroups (see also Problem 1).

Definition 4. A subgroup $H$ of a topological abelian group $X$ is TB-characterized if $H=s_{\mathbf{v}}(X)$, where $\mathbf{v} \in \widehat{X}^{\mathbb{N}}$ is a non-trivial TB-sequence.

The $N$-characterized subgroups are clearly closed, and they are always characterized as noted above. Every TB-characterized subgroup is obviously $T$-characterized. Moreover, every $T$-characterized subgroup is also $K$-characterized. Indeed, let $H=s_{\mathbf{v}}(X)$, where $\mathbf{v} \in \widehat{X}^{\mathbb{N}}$ is a non-trivial T-sequence, and without loss 
of generality, assume that $v_{n} \neq 0$ for all $n \in \mathbb{N}$; then, $\Gamma_{\mathbf{v}}^{\infty}=\varnothing$, that is $\mathbf{v}$ contains no constant subsequences, and so, $H$ is $K$-characterized.

Furthermore, proper dense characterized subgroups are TB-characterized by Lemma 1(ii), so also $T$-characterized and, in particular, $K$-characterized, but they are not $N$-characterized (as $N$-characterized subgroups are necessarily closed). We shall see below that closed (even open) subgroups need not be $K$-characterized in general. Denote by $\mathfrak{C h a r}_{K}(X)$ (respectively, $\mathfrak{C h a r}_{N}(X), \mathfrak{C h a r}_{T}(X), \mathfrak{C h a r}_{T B}(X)$ ) the family of all $\mathrm{K}$-characterized (respectively, $\mathrm{N}$-characterized, $\mathrm{T}$-characterized, $T B$-characterized) subgroups of the topological abelian group $X$. Then, we have the following strict inclusions:

$$
\mathfrak{C h a r}_{T B}(X) \subsetneq \mathfrak{C h a r}_{T}(X) \subsetneq \mathfrak{C h a r} \mathfrak{r}_{K}(X) \subsetneq \mathfrak{C h a r}(X) \supsetneq \mathfrak{C h a r}_{N}(X)
$$

We start giving some basic properties that can be proven immediately.

Corollary 2. Let $X$ be a topological abelian group and $X_{0}, X_{1}, X_{2}$ subgroups of $X$ with $X_{0} \leq X_{1} \leq X_{2}$ and such that $X_{1}$ is dually embedded in $X_{2}$.

(i) If $X_{0} \in \mathfrak{C h a r}{ }_{K}\left(X_{1}\right)$ and $X_{1} \in \mathfrak{C h a r}{ }_{K}\left(X_{2}\right)$, then $X_{0} \in \mathfrak{C h a r} \mathfrak{r}_{K}\left(X_{2}\right)$.

(ii) If $X_{0} \in \mathfrak{C h a r}_{N}\left(X_{1}\right)$ and $X_{1} \in \mathfrak{C h a r} \mathfrak{r}_{N}\left(X_{2}\right)$, then $X_{0} \in \mathfrak{C h a r}{ }_{N}\left(X_{2}\right)$.

Proof. (i) It suffices to note that if in the proof of Proposition $1, \mathbf{v}$ is one-to-one and $\mathbf{w}$ is one-to-one, then $\mathbf{w}^{*}$ is finitely many-to-one.

(ii) It suffices to note that if in the proof of Proposition $1, \Gamma_{\mathbf{v}}=\Gamma_{\mathbf{v}}^{\infty}$ and $\Gamma_{\mathbf{w}}=\Gamma_{\mathbf{w}}^{\infty}$, then also $\Gamma_{\mathbf{w}^{*}}=\Gamma_{\mathbf{w}^{*}}^{\infty}$.

By Lemma 8, we have directly the following:

Corollary 3. Every characterized subgroup of a topological abelian group $X$ is either $N$-characterized or it is the intersection of an N-characterized subgroup of $X$ and a K-characterized subgroup of $X$.

The following stability property is clear for $N$-characterized subgroups, while it is not known for characterized subgroups.

Lemma 9. Countable intersections of $N$-characterized subgroups are $N$-characterized.

The next correspondence theorem was proven in [16] for characterized subgroups of compact abelian groups.

Proposition 2. Let $X$ be a topological abelian group and $F$ a closed subgroup of $X$, and let $\pi: X \rightarrow X / F$ be the canonical projection. If $H$ is a characterized (respectively, 
K-characterized, N-characterized, T-characterized) subgroup of $X / F$, then $\pi^{-1}(H)$ is a characterized (respectively, K-characterized, $N$-characterized, T-characterized) subgroup of $X$.

Proof. Let $\mathbf{u}=\left(u_{n}\right) \in \widehat{X / F}^{\mathbb{N}}$, and consider $\widehat{\pi}: \widehat{X / F} \rightarrow \widehat{X}$. For every $n \in \mathbb{N}$, let $v_{n}=\widehat{\pi}\left(u_{n}\right)=u_{n} \circ \pi \in F^{\perp} \leq \widehat{X}$ and $\mathbf{v}=\left(v_{n}\right)$.

(i) Assume that $H=s_{\mathbf{u}}(X / F)$. Then, $\pi^{-1}(H)=s_{\mathbf{v}}(X)$, as $x \in s_{\mathbf{v}}(X)$ if and only if $v_{n}(x)=u_{n}(\pi(x)) \rightarrow 0$, and this occurs precisely when $\pi(x) \in H$.

(ii) Assume now that $H$ is $K$-characterized, that is assume that $H=s_{\mathbf{u}}(X / F)$ and that $\Gamma_{\mathbf{u}}^{\infty}=\varnothing$. By (i), $\pi^{-1}(H)=s_{\mathbf{v}}(X)$, and moreover, $\Gamma_{\mathbf{v}}^{\infty}=\varnothing$.

(iii) If $H$ is $N$-characterized, then assume that $H=n_{\mathbf{u}}(X / F)$. By (i), $\pi^{-1}(H)=$ $s_{\mathbf{v}}(X)$, and moreover, $\pi^{-1}(H)=n_{\mathbf{v}}(X)$, since $v_{n}(x)=u_{n}(\pi(x))=0$ for every $n \in \mathbb{N}$ precisely when $\pi(x) \in H=n_{\mathbf{u}}(X / F)$.

(iv) If $H$ is $T$-characterized, that is if $H=s_{\mathbf{u}}(X / F)$ and $\mathbf{u}$ is a $T$-sequence, it remains to verify that $\mathbf{v}$ is a $T$-sequence, as well, since $\pi^{-1}(H)=s_{\mathbf{v}}(X)$ by (i). Let $\tau$ be a Hausdorff group topology on $\widehat{X / F}$, such that $u_{n} \rightarrow 0$ in $(\widehat{X / F}, \tau)$. By Lemma 2(i), one can identify $\widehat{X / F}$ with the subgroup $F^{\perp}$ of $\widehat{X}$ by the algebraic monomorphism $\psi: \widehat{X / F} \rightarrow \widehat{X}$ defined by $\chi \mapsto \chi \circ \pi$. Let $\tau^{*}$ be the group topology on $\widehat{X}$ having as a local base at 0 the open neighborhoods of 0 in $(\widehat{X / F}, \tau)$. Then, $\tau^{*}$ is a Hausdorff group topology on $\widehat{X}$ and $v_{n} \rightarrow 0$ in $\left(\widehat{X}, \tau^{*}\right)$, as $v_{n}=\psi\left(u_{n}\right) \in F^{\perp}$ for every $n \in \mathbb{N}$ by definition.

Lemma 10. Let $X$ be a topological abelian group and $H$ a subgroup of $X$, such that $n(X) \leq H$. Then, $H$ is characterized (respectively, K-characterized, N-characterized, T-characterized) if and only if $H / n(X)$ is characterized (respectively, K-characterized, $N$-characterized, $T$-characterized).

Proof. Let $H=s_{\mathbf{v}}(X)$ for some $\mathbf{v} \in \widehat{X}^{\mathbb{N}}$, and denote by $\pi: X \rightarrow X / n(X)$ the canonical projection. For every $n \in \mathbb{N}$, since $n(X) \leq \operatorname{ker} v_{n}$, the character $v_{n}$ factorizes as $v_{n}=u_{n} \circ \pi$, where $u_{n} \in \widehat{X / n(X)}$. Then, $H / n(X)=s_{\mathbf{u}}(X / n(X))$. Vice versa, if $H / n(X)=s_{\mathbf{u}}(X / n(X))$ for some $\left.\mathbf{u} \in \widehat{X / n(X)}\right)^{\mathbb{N}}$, let $v_{n}=u_{n} \circ \pi$ for every $n \in \mathbb{N}$. Hence, $H=s_{\mathbf{v}}(X)$. Moreover, $\mathbf{v}$ is a finitely many-to-one sequence if and only if $\mathbf{u}$ is a finitely many-to-one sequence, so $H$ is $K$-characterized if and only if $H / n(X)$ is $K$-characterized. Similarly, $\Gamma_{\mathbf{u}}^{0}$ is finite, precisely when $\Gamma_{\mathbf{v}}^{0}$ is finite; hence, $H$ is $N$-characterized if and only if $H / n(X)$ is $N$-characterized.

It remains to check that $\mathbf{v}$ is a $T$-sequence precisely when $\mathbf{u}$ is a $T$-sequence. This follows from the fact that the natural homomorphism $\widehat{X / n(X)} \rightarrow \widehat{X}$ sending (the members of) $\mathbf{u}$ to (the members of) $\mathbf{v}$ is an isomorphism, so certainly preserving the property of being a $T$-sequence. 
The following lemma gives equivalent conditions for a subgroup to be characterized.

Lemma 11. Let $X$ be a topological abelian group and $H$ a subgroup of $X$. The following conditions are equivalent:

(i) $H \in \mathfrak{C h a r}(X)$;

(ii) there exists a closed subgroup $F$ of $X$, such that $F \leq H$ and $H / F \in \mathfrak{C h a r}(X / F)$;

(iii) there exists $\mathbf{v} \in \widehat{X}^{\mathbb{N}}$, such that for every closed $F \leq n_{\mathbf{v}}(X)$, one has that $H / F=$ $s_{\mathbf{u}}(X / F)$, where $\mathbf{u}=\left(u_{n}\right)$, and each $u_{n}$ is the factorization of $v_{n}$ through the canonical projection $\pi: X \rightarrow X / F$.

Proof. (iii) $\Rightarrow$ (ii) Take $F=n_{\mathbf{v}}(X)$.

(ii) $\Rightarrow$ (i) Since $F \leq H$, one has $H=\pi^{-1}(H / F)$, and one can conclude, by Proposition 2.

(i) $\Rightarrow$ (iii) Let $H=s_{\mathbf{v}}(X)$ for some $\mathbf{v} \in \widehat{X}^{\mathbb{N}}$ and $F \leq n_{\mathbf{v}}(X)$. Let $\pi: X \rightarrow X / F$ be the canonical projection. For every $n \in \mathbb{N}$, let $u_{n}$ be the character of $X / F$ defined by $\pi(x) \mapsto v_{n}(x)$. Then, $u_{n}$ is well defined, since $F \leq n_{\mathbf{v}}(X) \leq \operatorname{ker} v_{n}$. Hence, $u_{n} \circ \pi=v_{n}$ for every $n \in \mathbb{N}$, and $H / F=s_{\mathbf{u}}(X / F)$. Indeed, for every $h \in H$, we have $u_{n}(\pi(h))=v_{n}(h) \rightarrow 0$, and hence, $H / F \leq s_{\mathbf{u}}(X / F)$. Conversely, if $\pi(x) \in s_{\mathbf{u}}(X / F)$, then $v_{n}(x)=u_{n}(\pi(x)) \rightarrow 0$. Hence, $x \in H$, and so, $\pi(x) \in H / F$.

\section{Auto-Characterized Groups}

The following consequence of [16, Proposition 2.5] motivates the introduction of the notion of auto-characterized group (see Definition 5).

Proposition 3. Let $X$ be a compact abelian group. Then, $s_{\mathbf{v}}(X)=X$ for some $\mathbf{v} \in \widehat{X}^{\mathbb{N}}$ if and only if the sequence $\mathbf{v}$ is trivially null.

Proof. It is clear from (2) that $s_{\mathbf{v}}(X)=X$ if $\mathbf{v}$ is trivially null. Assume now that $X=s_{\mathbf{v}}(X)$ for some $\mathbf{v} \in \widehat{X}^{\mathbb{N}}$. By [16, Proposition 2.5], being $s_{\mathbf{v}}(X)$ compact, there exists $m \in \mathbb{N}$, such that $X=s_{\mathbf{v}}(X)=n_{\mathbf{v}_{(m)}}(X)$, and so, $v_{n}=0$ for all $n \geq m$.

If one drops the compactness, then the conclusion of Proposition 3 may fail, as shown in the next example.

\section{Example 1.}

(i) Let $N$ be an infinite countable subgroup of $\mathbb{T}$. As mentioned in the Introduction, $N$ is characterized in $\mathbb{T}$; hence, $N=s_{\mathbf{v}}(\mathbb{T})$ for a non-trivial sequence $\mathbf{v} \in \mathbb{Z}^{\mathbb{N}}$. If $\mathbf{u}=\mathbf{v} \uparrow_{N}$, then $\mathbf{u}$ is non-trivial (since $N$ is dense in $\mathbb{T}$ ), and $s_{\mathbf{u}}(N)=N$. 
(ii) Let $X=\mathbb{R}$, let $\pi: \mathbb{R} \rightarrow \mathbb{T}$ be the canonical projection and let $\mathbf{v}=\left(v_{n}\right) \in \widehat{\mathbb{R}}^{\mathbb{N}}$, such that $v_{0}=0$ and $v_{n}(x)=\pi\left(\frac{x}{n}\right) \in \mathbb{T}$ for every $x \in \mathbb{R}$ and $n \in \mathbb{N}_{+}$. Obviously, $s_{\mathbf{v}}(\mathbb{R})=\mathbb{R}$, even though $\mathbf{v}$ is non-trivial.

(iii) Let $X=\mathbb{Q}_{p}$, where $p$ is a prime. For every $n \in \mathbb{N}$, let $v_{n}=p^{n} \in \widehat{\mathbb{Q}_{p}}$. Obviously, $s_{\mathbf{v}}\left(\mathbb{Q}_{p}\right)=\mathbb{Q}_{p}$, even though $\mathbf{v}$ is non-trivial.

Motivated by Proposition 3 and Example 1, we give the following:

Definition 5. A topological abelian group $X$ is auto-characterized if $X=s_{\mathbf{v}}(X)$ for some non-trivial $\mathbf{v} \in \widehat{X}^{\mathbb{N}}$.

Items (ii) and (iii) of Example 1 show that $\mathbb{R}$ and $\mathbb{Q}_{p}$ are auto-characterized.

Remark 3. (i) Let $X$ be an auto-characterized group, so let $\mathbf{v} \in \widehat{X}^{\mathbb{N}}$ be non-trivial and such that $X=s_{\mathbf{v}}(X)$. Then, there exists a one-to-one subsequence $\mathbf{u}$ of $\mathbf{v}$, such that $u_{n} \neq 0$ for every $n \in \mathbb{N}$ and $X=s_{\mathbf{u}}(X)$.

Indeed, if $\chi \in \Gamma_{\mathbf{v}}^{\infty}$, then $X=s_{\mathbf{v}}(X) \leq \operatorname{ker} \chi$, and so, $\chi=0$; therefore, $\Gamma_{\mathbf{v}}^{\infty}$ is either empty or $\{0\}$. Since $\mathbf{v}$ is non-trivial, $\Gamma_{\mathbf{v}}^{0}$ is infinite; hence, $X=s_{\mathbf{v}}(X)=s_{\mathbf{v}^{0}}(X)$ by Lemma 8 (ii). Let $\mathbf{u}$ be the one-to-one subsequence of $\mathbf{v}^{0}$, such that $\Gamma_{\mathbf{u}}=\Gamma_{\mathbf{v}^{0}}$; therefore, $X=s_{\mathbf{u}}(X)$.

(ii) The above item shows that auto-characterized groups are $K$-characterized subgroups of themselves. However, one can prove actually that they are $T$-characterized subgroups of themselves (indeed, TB-characterized subgroups of themselves; see [24]).

\subsection{Basic Properties of Auto-Characterized Groups}

We start by a direct consequence of Lemma 10:

Lemma 12. Let $X$ be a topological abelian group and $H$ a subgroup of $X$, such that $n(X) \leq H$. Then, $H$ is auto-characterized if and only if $H / n(X)$ is auto-characterized.

The next proposition, describing an auto-characterized group in terms of the null sequences of its dual, follows from the definitions and Lemma 1:

Proposition 4. A topological abelian group $X$ is auto-characterized if and only if $(\widehat{X}, \sigma(\widehat{X}, X))$ has a non-trivial null sequence $\mathbf{v}$ (in such a case, $X=s_{\mathbf{v}}(X)$ ).

In the next lemma, we see when a subgroup of an auto-characterized group is auto-characterized, and vice versa.

Lemma 13. Let $X$ be a topological abelian group and $H$ a subgroup of $X$. 
(i) If $X$ is auto-characterized and $H$ is dense in $X$, then $H$ is auto-characterized.

(ii) If $H$ is auto-characterized and one of the following conditions holds, then $X$ is auto-characterized:

(a) $H$ is a topological direct summand of $X$;

(b) $H$ is open and has a finite index.

Proof. (i) Let $X=s_{\mathbf{v}}(X)$ for $\mathbf{v} \in \widehat{X}$ with $v_{n} \neq 0$ for every $n \in \mathbb{N}$, and let $u_{n}=v_{n} \uparrow_{H} \in \widehat{H}$. Then, each $u_{n}$ is non-zero and $H=s_{\mathbf{u}}(H)$.

(ii) Let $H=s_{\mathbf{v}}(H)$ for some $\mathbf{v} \in \widehat{H}^{\mathbb{N}}$ with $v_{n} \neq 0$ for every $n \in \mathbb{N}$.

(a) Let $X=H \times Z$. For every $n \in \mathbb{N}$, let $u_{n}$ be the unique character of $X$ that extends $v_{n}$ and such that $u_{n}$ vanishes on $Z$. Then, $u_{n} \neq 0$ for every $n \in \mathbb{N}$, and $X=s_{\mathbf{u}}(X)$.

(b) Arguing by induction, we can assume without loss of generality that $[X: H]=p$ is prime. Let $X=H+\langle x\rangle$ with $x \notin H$ and $p x \in H$. If $p x=0$, then $H$ is an open direct summand of $X$, so $H$ is also a topological direct summand of $X$; hence, item (a) applies. Assume now that $p x \neq 0$, and let $a_{n}=v_{n}(p x)$ for every $n \in \mathbb{N}$. If $a_{n}=0$ for infinitely many $n$, extend $v_{n}$ to $u_{n} \in \widehat{X}^{\mathbb{N}}$ for those $n$ by letting $u_{n}(x)=0$. Then, obviously, the sequence $\mathbf{u}$ obtained in this way is not trivially null and $X=s_{\mathbf{u}}(X)$, so $X$ is auto-characterized. Assume now that $a_{n} \neq 0$ for infinitely many $n \in \mathbb{N}$; for those $n$, pick an element $b_{n} \in \mathbb{T}$ with $p b_{n}=a_{n}$, and extend $v_{n}$ by letting $u_{n}(h+k x)=v_{n}(h)+k b_{n}$. Let $w_{n}=p u_{n}$. Then, $w_{n}(x)=a_{n} \neq 0$, so $\mathbf{w}$ is not trivially null. Moreover, $X=s_{\mathbf{u}}(X)$ as $p X \leq H$.

Lemma 14. Let $X$ be a topological abelian group and $\mathbf{v} \in \widehat{X}^{\mathbb{N}}$. If $F$ is a subgroup of $X$, such that $F \leq s_{\mathbf{v}}(X)$ and $F$ is not auto-characterized, then $F \leq n_{\mathbf{v}_{(m)}}(X)$ for some $m \in \mathbb{N}$.

Proof. Let $u_{n}=v_{n} \uparrow_{F}$ for every $n \in \mathbb{N}$ and $\mathbf{u}=\left(u_{n}\right) \in \widehat{F}^{\mathbb{N}}$. Then, $F=s_{\mathbf{u}}(F)$, so the sequence $\mathbf{u}$ must be trivially null. Let $m \in \mathbb{N}$, such that $u_{n}=0$ for every $n \geq m$. Therefore, $F \leq n_{\mathbf{v}_{(m)}}(X)$.

The following consequence of Lemma 14 is a generalization of Lemma 2.6 in [16], where the group $X$ is compact.

Corollary 4. Let $X$ be a topological abelian group, $F$ and $H$ subgroups of $X$, such that $F$ is compact and $F \leq H$. Then, $H / F \in \mathfrak{C h a r}(X / F)$ if and only if $H \in \mathfrak{C h a r}(X)$.

Proof. Denote by $\pi: X \rightarrow X / F$ the canonical projection. If $H / F$ is a characterized subgroup of $X / F$, then $H=\pi^{-1}(H / F)$ is a characterized subgroup of $X$ by Proposition 2. Assume now that $H=s_{\mathbf{v}}(X)$ for some $\mathbf{v} \in \widehat{X}^{\mathbb{N}}$. Since $F$ is compact, Proposition 3 implies that $F$ is not auto-characterized. By Lemma $14, F$ is contained in $n_{\mathbf{v}_{(m)}}(X)$ for a sufficiently large $m \in \mathbb{N}$. Let $\pi^{\prime}: X / F \rightarrow X / n_{\mathbf{v}_{(m)}}(X)$ be the canonical 
projection and $q=\pi^{\prime} \circ \pi$, then $q^{-1}\left(H / n_{\mathbf{v}_{(m)}}(X)\right)=H=s_{\mathbf{v}}(X)=s_{\mathbf{v}_{(m)}}(X)$. Therefore, one deduces from Lemma 11 that $H / n_{\mathbf{v}_{(m)}}(X)$ is a characterized subgroup of $X / n_{\mathbf{v}_{(m)}}(X)$. Hence, by Proposition $2, H / F=\left(\pi^{\prime}\right)^{-1}\left(H / n_{\mathbf{v}_{(m)}}(X) \in \mathfrak{C h a r}(X / F)\right.$.

The argument of the above proof fails in case $F$ is not compact. For example, take $F=H=X=N$, where $N$ is as in Example 1; then, one cannot conclude that $\mathbf{v} \uparrow_{F}$ is trivially null and, hence, that $F$ is contained in $n_{\mathbf{v}_{(m)}}(X)$.

\subsection{Criteria Describing Auto-Characterized Groups}

Here, we give two criteria for a group to be auto-characterized. We start below with a criterion for locally compact abelian groups, while a general one, in terms of the Bohr compactification, will be given at the end of the section.

We established in Proposition 3 that no compact abelian group is auto-characterized; now, we prove in Theorem 3 that this property describes the compact abelian groups within the larger class of all locally compact abelian groups. This follows easily from Lemma 13(ii) for the locally compact abelian groups that contain a copy of $\mathbb{R}$, while the general case requires the following deeper argument.

Theorem 3. If $X$ is a locally compact abelian group, then $X$ is auto-characterized if and only if $X$ is not compact.

Proof. If $X$ is auto-characterized, then $X$ is not compact according to Proposition 3. Assume now that $X$ is not auto-characterized. Then, by Fact 2 and Proposition 4 , the dual $\widehat{X}$ has no non-trivial null sequences in its Bohr topology. However, since $\widehat{X}$ is locally compact, it has the same null sequences as its Bohr modification $\widehat{X}^{+}$. Therefore, $\widehat{X}$ is a locally compact group without non-trivial null sequences. We have to conclude that $X$ is compact.

This follows from the conjunction of several facts. The first one is the deep result that non-discrete locally compact abelian groups have non-trivial null sequences. (This follows, in turn, from that fact that a non-discrete locally compact abelian group either contains a line $\mathbb{R}$ or an infinite compact subgroup. Since compact groups are dyadic compacts, i.e., continuous images of Cantor cubes, they have non-trivial null sequences.) Now, we can conclude that the locally compact group $\widehat{X}$ is discrete. It is a well known fact that this implies the compactness of $X$.

Remark 4. An alternative argument to prove that non-discrete locally compact abelian groups have non-trivial null sequences is based on a theorem by Hagler, Gerlits and Efimov (proven independently also by Efimov in [25]). It states that every infinite compact group $K$ contains a copy of the Cantor cube $\{0,1\}^{w(K)}$, which obviously has plenty of non-trivial null sequences. 
In order to obtain a general criterion describing auto-characterized groups we need another relevant notion in the theory of characterized subgroups:

Definition 6. [1] Let $X$ be a topological abelian group and $H$ a subgroup of $X$. Let:

$$
\mathfrak{g}_{X}(H)=\bigcap\left\{s_{\mathbf{v}}(X): \mathbf{v} \in \widehat{X}^{\mathbb{N}}, H \leq s_{\mathbf{v}}(X)\right\} .
$$

A subgroup $H$ of $X$ is said to be:

(i) $\mathfrak{g}$-dense if $\mathfrak{g}_{X}(H)=X$;

(ii) $\mathfrak{g}$-closed if $\mathfrak{g}_{X}(H)=H$.

We write simply $\mathfrak{g}(H)$ when there is no possibility of confusion. Clearly, $\mathfrak{g}(H)$ is a subgroup of $X$ containing $H$. Moreover, $\mathfrak{g}(\{0\})$ is the intersection of all characterized subgroups of $X$ and $\mathfrak{g}(\{0\}) \leq n(X)$.

Remark 5. Let $X=(X, \tau)$ be a topological abelian group and $H$ a subgroup of $X$.

(i) If $X_{1}$ is another topological abelian group and $\phi: X \rightarrow X_{1}$ a continuous homomorphism, then $\phi\left(\mathfrak{g}_{X}(H)\right) \leq \mathfrak{g}_{X_{1}}(\phi(H))$ (see [1, Proposition 2.6]).

(ii) Moreover, $\mathfrak{g}_{X}(H) \leq \bar{H}^{\tau^{+}}$. Indeed, $\bar{H}^{\tau^{+}}=\bigcap\{\operatorname{ker} \chi: \chi \in \widehat{X}, H \leq \operatorname{ker} \chi\}$ and $\operatorname{ker} \chi=n_{\mathbf{v}}(X)=s_{\mathbf{v}}(X)$ for $\mathbf{v} \in \widehat{X}^{\mathbb{N}}$ with $\Gamma_{\mathbf{v}}=\Gamma_{\mathbf{v}}^{\infty}=\{\chi\}$ (i.e., $\mathbf{v}$ is the constant sequence given by $\chi$ ). Item (i) says, in terms of $[15,26]$, that $\mathfrak{g}$ is a closure operator in the category of topological abelian groups. The inclusion $\mathfrak{g}_{X}(H) \leq \bar{H}^{\tau^{+}}$says that $\mathfrak{g}$ is finer than the closure operator defined by $H \mapsto \bar{H}^{\tau^{+}}$.

(iii) If $H$ is dually closed, then $H$ is $\mathfrak{g}$-closed by item (ii).

(iv) If $(X, \tau)$ is a locally compact abelian group, then every closed subgroup of $(X, \tau)$ is dually closed, and so, (ii) implies that $\mathfrak{g}(H) \leq \bar{H}^{\tau}$ for every subgroup $H$ of $X$. Therefore, $\mathfrak{g}$-dense subgroups are also dense in this case.

(v) The inclusion $\mathfrak{g}(H) \leq \bar{H}$ may fail if the group $H$ is not MAP (e.g., if $X$ is MinAP, then $\mathfrak{g}(H)=X$ for every $H$, while $X$ may have proper closed subgroups).

The next result shows that the auto-characterized precompact abelian groups are exactly the dense non-g-dense subgroups of the compact abelian groups.

Theorem 4. Let X be a precompact abelian group. The following conditions are equivalent:

(i) $X$ is auto-characterized;

(ii) $X$ is not $\mathfrak{g}$-dense in its completion $\widetilde{X}$.

Proof. (ii) $\Rightarrow$ (i) Assume that $X$ is not $\mathfrak{g}$-dense in $K:=\widetilde{X}$. Then, there exists a sequence $\mathbf{v} \in \widehat{K}^{\mathbb{N}}$, such that $X \leq s_{\mathbf{v}}(K)<K$. By Proposition 3 (see also Remark 3), we may 
assume without loss of generality that $v_{n} \neq 0$ for every $n \in \mathbb{N}$. Let $u_{n}=v_{n} \uparrow_{X}$ for every $n \in \mathbb{N}$. Since $X$ is dense in $K$, clearly $u_{n} \neq 0$ for every $n \in \mathbb{N}$. Moreover, $X=s_{\mathbf{u}}(X)$; hence, $X$ is auto-characterized.

(i) $\Rightarrow$ (ii) Suppose that $X$ is auto-characterized, say $X=s_{\mathbf{u}}(X)$ for $\mathbf{u} \in \widehat{X}^{\mathbb{N}}$, such that $u_{n} \neq 0$ for every $n \in \mathbb{N}$. For every $n \in \mathbb{N}$, let $v_{n} \in \widehat{K}$ be the extension of $u_{n}$ to $K$. Then, $X \leq s_{\mathbf{v}}(K)<K$ by Proposition 3, so $X$ is not $\mathfrak{g}$-dense in $K$.

If $X$ is a MAP abelian group, then $\tau^{+}$is precompact, and the Bohr compactification of $X$ is $r_{X}: X \rightarrow b X$, where $b X$ is the completion of $\left(X, \tau^{+}\right)$and $r_{X}$ is an injective homomorphism. If $X$ is not MAP, then $n(X) \neq\{0\}$. Consider the quotient $X / n(X)$, which is a MAP group. Then, take the Bohr compactification $r_{X / n(X)}: X / n(X) \rightarrow b(X / n(X))$. The Bohr compactification of $X$ is $r_{X}: X \rightarrow b X$, where $b X:=b(X / n(X))$ and $r_{X}=r_{X / n(X)} \circ \pi$, where $\pi: X \rightarrow X / n(X)$ is the canonical projection.

Corollary 5. Let $X$ be a MAP abelian group. The following conditions are equivalent:

(i) $X$ is auto-characterized;

(ii) $X$ is not $\mathfrak{g}$-dense in $b X$.

Proof. Since $X$ is MAP, $X$ embeds in $b X$. By Lemma $6, X$ and $X^{+}$have the same characterized subgroups. Moreover, $X^{+}$is precompact, and by definition, $b X$ is the completion of $X^{+}$. Then, it suffices to apply Theorem 4 .

Theorem 5. Let X be a topological abelian group. The following conditions are equivalent:

(i) $\mathrm{X}$ is auto-characterized;

(ii) $r_{X}(X)$ is not $\mathfrak{g}$-dense in $b X$.

Proof. Since $X$ is auto-characterized precisely when $X / n(X)$ is auto-characterized by Lemma 12, apply Corollary 5 to conclude.

\section{K-Characterized Subgroups}

We start by recalling [23, Lemma 3.19]: if $X$ is a compact abelian group and $\mathbf{v} \in \widehat{X}^{\mathbb{N}}$ is a one-to-one sequence, then $s_{\mathbf{v}}(X)$ has Haar measure zero in $X$. Since $K$-characterized subgroups are characterized by finitely many-to-one sequences (which obviously contain a one-to-one subsequence), this result applies to K-characterized subgroups and gives the following (formally weaker) result, which will be necessary and more convenient to apply in the current paper:

Lemma 15. If $X$ is a compact abelian group and $H \in \mathfrak{C h a r}_{K}(X)$, then $H$ has Haar measure zero (hence, $[X: H]$ is infinite). In particular, no open subgroup of $X$ is K-characterized. 
in $\widehat{\mathbb{T}}^{\mathbb{N}}$.

Lemma 15 cannot be inverted; take, for example, the constant sequence $\mathbf{u}=(1)$

Remark 6. If $X$ is a connected compact abelian group, then the conclusion of Lemma 15 holds for all non-trivial sequences in $\widehat{X}$, since every measurable proper subgroup $H$ of $X$ has measure zero (indeed, $X$ is divisible, so the proper subgroup $H$ of $X$ has infinite index; hence, the measure of $H$ must be 0 , as $X$ has measure 1 ).

Example 2. Here, we provide examples of non-auto-characterized non-compact abelian groups.

(i) A relatively simple example can be obtained by taking a dense non-measurable subgroup $X$ of a connected compact abelian group $K$. Since we intend to deduce that $X$ is not auto-characterized by using Theorem 4 , we have to check that $X$ is $\mathfrak{g}$-dense in $K$. Indeed, every measurable proper subgroup of $K$ has measure zero as noted in Remark 6; therefore, every proper characterized (hence, every non-g-dense) subgroup of $K$ has measure zero. Therefore, $X$ is not contained in any proper characterized subgroup of $K$, i.e., $X$ is $\mathfrak{g}$-dense in $K$.

(ii) More sophisticated examples of $\mathfrak{g}$-dense subgroups of metrizable compact abelian groups were given in [27] (under the assumption of Martin Axiom) and in [19] (in ZFC). These groups have the additional property of being of measure zero (so that the above elementary argument cannot be used to verify the $\mathfrak{g}$-density).

\subsection{When Closed Subgroups of Infinite Index are K-Characterized}

The next theorem gives a sufficient condition (see item (iii)) for a closed subgroup of infinite index $H$ to be $K$-characterized. This condition implies, as a by-product, that $H$ is also $N$-characterized.

The easier case of open subgroups will be tackled in Theorem 7, by applying Theorem 6.

Theorem 6. Let $X$ be a topological abelian group and $H$ a closed subgroup of $X$ of infinite index. The following conditions are equivalent:

(i) $H \in \mathfrak{C h a r}_{K}(X) \cap \mathfrak{C h a r} \mathfrak{r}_{N}(X)$;

(ii) $H \in \mathfrak{C h a r}_{N}(X)$;

(iii) $X / H$ is $M A P$, and $(\widehat{X / H}, \sigma(\widehat{X / H}, X / H))$ is separable.

Proof. (i) $\Rightarrow$ (ii) is obvious.

(ii) $\Rightarrow$ (iii) Since $H$ is $N$-characterized, then $H$ is dually closed by Lemma 7(ii), that is $X / H$ is MAP. Let $H=n_{\mathbf{v}}(X)$ for some $\mathbf{v} \in \widehat{X}^{\mathbb{N}}$, and let $\pi: X \rightarrow X / H$ be the canonical projection. Since $\operatorname{ker} \pi=H \leq \operatorname{ker} v_{n}$ for every $n \in \mathbb{N}$, one can factorize 
$v_{n}: X \rightarrow \mathbb{T}$ through $\pi$, i.e., write $v_{n}=\bar{v}_{n} \circ \pi$ for appropriate $\bar{v}_{n} \in \widehat{X / H}$. It remains to verify that $D=\left\{\bar{v}_{n}: n \in \mathbb{N}\right\}$ is dense in $(\widehat{X / H}, \sigma(\widehat{X / H}, X / H))$. To this end, let $\bar{y}=\pi(y) \in X / H$; if $\xi_{\bar{y}}(D)=\{0\}$, then $\bar{v}_{n}(\bar{y})=v_{n}(y)=0$, and so, $y \in H$, that is, $\bar{y}=0$.

(iii) $\Rightarrow$ (i) Let $Y=X / H$, equipped with the quotient topology. By hypotheses, $Y$ is infinite and MAP, while $\widehat{Y}$ is an infinite topological abelian group with a countably infinite dense subgroup $D$. According to Proposition 2 applied to the canonical projection $\pi: X \rightarrow Y$, it suffices to prove that $\{0\}$ is a $K$-characterized subgroup of $Y$. Let $D=\left\{v_{n}: n \in \mathbb{N}\right\}$ be a one-to-one enumeration of $D$ and $\mathbf{v}=\left(v_{n}\right)$. To prove that $s_{\mathbf{v}}(Y)=\{0\}$, we have to show that for every non-zero $y \in Y$, there exists a neighborhood $U$ of 0 in $\mathbb{T}$, such that $v_{n}(y) \notin U$ for infinitely many $n \in \mathbb{N}$. Actually, we show that $U=\mathbb{T}_{+}$works for all non-zero $y \in Y$. In fact, for every $y \in Y \backslash\{0\}$, one has that $N_{y}:=\{d(y): d \in D\}=\left\{v_{n}(y): n \in \mathbb{N}\right\}$ is a non-trivial subgroup of $\mathbb{T}$, as $Y$ is MAP and $y \neq 0$.

Let $y \in Y \backslash\{0\}$. If $N_{y}$ is infinite, then $N_{y}$ is dense in $\mathbb{T}$; so, $N_{y} \backslash U$ is infinite, and we are done. Now, consider the case when $N_{y}$ is finite. As $N_{y} \neq\{0\}$ and $U$ contains no non-trivial subgroups of $\mathbb{T}$, there exists $a \in N_{y}$, such that $a \notin U$. Then, the map $f_{y}: D \rightarrow \mathbb{T}$ defined by $f_{y}(d)=d(y)$ is a homomorphism with $f_{y}(D)=N_{y}$ finite. Therefore, $K:=\operatorname{ker} f_{y}$ is a finite-index subgroup of $D$, so $K$ is infinite. Let $a=v_{m}(y)$ for some $m \in \mathbb{N}$. Then, $v_{m}+K=\{d \in D: d(y)=a\}$ is infinite, as well. This means that $v_{n}(y)=a \notin U$ for infinitely many $n$ (namely, those $n$ for which $v_{n} \in v_{m}+K$ ). Therefore, $v_{n}(y) \nrightarrow \rightarrow$, and so, $y \notin s_{\mathbf{v}}(Y)$.

Finally, let us note that the above argument shows also that $H$ is $N$-characterized, as obviously $H \leq n_{\mathbf{v}}(X)$.

The following is an obvious consequence of Theorem 6 .

Corollary 6. Let $X$ be a topological abelian group and $H$ a closed subgroup of $X$ of infinite index. If $H \in \mathfrak{C h a r}_{N}(X)$, then $H \in \mathfrak{C h a r} \mathfrak{r}_{K}(X)$.

Next, we rewrite Theorem 6 in the case of locally compact abelian groups.

Corollary 7. Let $X$ be a locally compact abelian group and $H$ a subgroup of $X$. Then, $H \in \mathfrak{C h a r}_{N}(X)$ if and only if $H$ is closed and $\widehat{X / H}$ is separable.

Proof. As both conditions imply that $H$ is closed, we assume without loss of generality that $H$ is closed. Since $X / H$ and $\widehat{X / H}$ are locally compact abelian groups, $X / H$ is MAP, and the Bohr topology on $\widehat{X / H}$ coincides with $\sigma(\widehat{X / H}, X / H)$ by Fact 2, so the separability of $\widehat{X / H}$ is equivalent to the separability of $\widehat{X / H}^{+}$by Fact 1 . If $H$ has a infinite index in $X$, apply Theorem 6 to conclude. If $H$ has a finite index in $X$, 
then the equivalence is trivially satisfied; indeed, $H$ is a finite intersection of kernels of characters, so it is $N$-characterized, and $\widehat{X / H}$ is finite, so separable.

As a consequence of Theorem 6, we find a sufficient condition for an open subgroup of infinite index to be $K$-characterized:

Theorem 7. Let $X$ be a topological abelian group and $H$ an open subgroup of $X$ of infinite index. Then, the following conditions are equivalent:

(i) $H \in \mathfrak{C h a r}(X)$;

(ii) $H \in \mathfrak{C h a r} \mathfrak{r}_{K}(X)$;

(iii) $[X: H] \leq \mathfrak{c}$;

(iv) $\widehat{X / H}$ is separable.

Proof. (ii) $\Rightarrow$ (i) is clear, and (i) $\Rightarrow$ (iii) is Corollary 1 .

(iii) $\Rightarrow$ (iv) Since $\widehat{X / H}$ is a compact abelian group of weight at most $\mathfrak{c}$, it is separable by Lemma 3.

(iv) $\Rightarrow$ (ii) As $[X: H]$ is infinite, we can apply Theorem 6 to conclude that $H$ is K-characterized.

The following is another direct consequence of Theorem 6.

Corollary 8. If $X$ is a metrizable compact abelian group, then every closed non-open subgroup of $X$ is K-characterized.

\subsection{When Closed Subgroups of Finite Index are K-Characterized}

We start by giving the following useful technical lemma.

Lemma 16. Let $X$ be a topological abelian group and $H$ an open subgroup of $X$, such that $X=H+\langle x\rangle$ for some $x \in X \backslash H$. If $H$ is auto-characterized, then $H \in \mathfrak{C h a r}_{K}(X)$.

Proof. Let $\mathbf{u} \in \widehat{H}^{\mathbb{N}}$, such that $H=s_{\mathbf{u}}(H)$. By Remark 3, we can assume that $\mathbf{u}$ is one-to-one and that $u_{n} \neq 0$ for every $n \in \mathbb{N}$.

Assume first that $H \cap\langle x\rangle=\{0\}$. If $o(x)$ is infinite, then fix an irrational number $\alpha \in \mathbb{R}$, and for every $n \in \mathbb{N}$, let $v_{n}(x)=\alpha+\mathbb{Z}$ and $v_{n}(h)=u_{n}(h)$ for every $h \in H$. If $o(x)=k$ is finite, then for every $n \in \mathbb{N}$, let $v_{n}(x)=\frac{1}{k}+\mathbb{Z}$ and $v_{n}(h)=u_{n}(h)$ for every $h \in H$. In both cases, it is straightforward to prove that $H=s_{\mathbf{v}}(X)$. Moreover, since $\mathbf{u}$ is one-to-one, then also $\mathbf{v}$ is one-to-one.

Suppose now that $H \cap\langle x\rangle=\langle m x\rangle$ for some $m \in \mathbb{N}$, with $m \geq 1$. As $x \notin H$, one has $m \geq 2$. 
For every $n \in \mathbb{N}$, let $a_{n}=u_{n}(m x) \in \mathbb{T}$. Since $u_{n}(m x) \rightarrow 0$, there exists $n_{0}$, such that $\left\|a_{n}\right\|<\frac{1}{m^{2}}$ for every $n \geq n_{0}$. As $s_{\mathbf{u}_{\left(n_{0}\right)}}(H)=H$, we shall assume for simplicity that $\left\|a_{n}\right\|<\frac{1}{m^{2}}$ every $n \in \mathbb{N}$.

Claim 1. For every $a \in \mathbb{T}$ with $\|a\|<\frac{1}{m^{2}}$, there exists $b \in \mathbb{T}$, such that

$$
m b=a \text { and }\|k b\|>\frac{1}{m^{2}} \text { for every } k \in \mathbb{N}, 1 \leq k<m .
$$

Proof. We tackle the problem in $\mathbb{R}$, that is identifying $\mathbb{T}$ with $[0,1)$. First, assume that $0 \leq a<\frac{1}{m^{2}}$, and let:

$$
b=\frac{a}{m}+\frac{1}{m}
$$

Then, $m b=a+1 \equiv_{\mathbb{Z}} a$ and $\frac{1}{m} \leq b \leq \frac{2}{m}$. Let now $k \in \mathbb{N}$ with $1 \leq k \leq m-1$, then:

$$
\frac{1}{m^{2}}<\frac{k}{m} \leq k b=k \frac{a}{m}+\frac{k}{m}<\frac{m-1}{m^{2}}+\frac{m-1}{m}=1-\frac{1}{m^{2}}
$$

Therefore, $\|k b\|>\frac{1}{m^{2}}$. This establishes condition (5) in the current case.

It remains to consider the case $\frac{m^{2}-1}{m^{2}}<a<1$. Let $a^{*}=1-a$, i.e., $a^{*}=-a$ in $\mathbb{T}$. Then, obviously, $\left\|a^{*}\right\|<\frac{1}{m^{2}}$ and $0 \leq a^{*}<\frac{1}{m^{2}}$. Hence, by the above case applied to $a^{*}$, there exists $b^{*} \in \mathbb{T}$ satisfying condition (5) with $-a$ in place of $a\left(\right.$ i.e., $\left.m b^{*}=-a\right)$. Let $b=-b^{*} \in \mathbb{T}$. Then, condition (5) holds true for $b$ and $a$, as $\|k(-b)\|=\|k b\|$ for every $k \in \mathbb{N}$ with $1 \leq k<m$.

For every $n \in \mathbb{N}$, apply Claim 1 to $a_{n}$ to get $b_{n}$ as in Equation (5), then define $v_{n}: X \rightarrow \mathbb{T}$ by letting $v_{n}(x)=b_{n}$ for every $n \in \mathbb{N}$ and $v_{n}(h)=u_{n}(h)$ for every $h \in H$. As $u_{n}(m x)=v_{n}(m x)=m v_{n}(x)=m b_{n}=a_{n}$, this definition is correct. Moreover, since $H$ is open in $X, v_{n} \in \widehat{X}$. Since $\mathbf{u}$ is one-to-one, then $\mathbf{v}=\left(v_{n}\right)$ is one-to-one, too.

We show that

$$
v_{n}(k x) \rightarrow 0 \text { for } k \in \mathbb{N} \text { if and only if } k \in m \mathbb{Z} .
$$

In fact, if $k=k^{\prime} m$ for some $k^{\prime} \in \mathbb{N}$,

$$
v_{n}(k x)=k^{\prime} v_{n}(m x)=k^{\prime} a_{n} \rightarrow 0 .
$$

The other way around, assume that $k=k^{\prime} m+r$, where $k^{\prime} \in \mathbb{N}$ and $1 \leq r \leq m-1$. Then,

$$
v_{n}(k x)=k^{\prime} v_{n}(m x)+r v_{n}(x)=k^{\prime} a_{n}+r b_{n} \nrightarrow \nrightarrow 0
$$

since $k^{\prime} a_{n} \rightarrow 0$ and $\left\|r b_{n}\right\| \geq \frac{1}{m^{2}}$ by Equation (5). 
We deduce finally that $H=s_{\mathbf{v}}(X)$. Indeed, $H=s_{\mathbf{u}}(H) \leq s_{\mathbf{v}}(X)$, so it remains to prove that $s_{\mathbf{v}}(X) \leq H$. To this end, let $y \in X \backslash H$, that is $y=h+k x$ for some $h \in H$ and $k \in \mathbb{N}$ with $1 \leq k<m$. Then,

$$
v_{n}(y)=v_{n}(h+k x)=u_{n}(h)+v_{n}(k x) .
$$

Since $h \in H=s_{\mathbf{u}}(H)$, that is, $u_{n}(h) \rightarrow 0$, while $v_{n}(k x) \not \rightarrow 0$ by condition (7), we conclude that $v_{n}(y) \nrightarrow \rightarrow$, that is, $y \notin s_{\mathbf{v}}(X)$. Hence, $H=s_{\mathbf{v}}(X)$.

Every open finite-index subgroup is a finite intersection of kernels of characters, so it is $\mathrm{N}$-characterized. In the next theorem we describe when a proper open finite-index subgroup is $\mathrm{K}$-characterized.

Theorem 8. Let $X$ be a topological abelian group and $H$ a proper open subgroup of $X$ of finite index. Then, $H \in \mathfrak{C h a r}_{K}(X)$ if and only if $H$ is auto-characterized.

Proof. Assume that $H \in \mathfrak{C h a r}_{K}(X)$. We can write $H=s_{\mathbf{u}}(X)$ for $\mathbf{u} \in \widehat{X}^{\mathbb{N}}$ one-to-one. Let $v_{n}=u_{n} \uparrow_{H}$ for every $n \in \mathbb{N}$. Then, the map $u_{n} \mapsto v_{n}$ is finitely many-to-one, as $X / H$ is finite. Therefore, $\mathbf{v}=\left(v_{n}\right)$ is finitely many-to-one. Obviously, $H=s_{\mathbf{v}}(H)$, so $H$ is auto-characterized.

Now, assume that $H$ is auto-characterized. Since $H$ has finite index in $X$, there exist $x_{1}, \ldots, x_{n} \in X$, such that $X=H+\left\langle x_{1}, \ldots, x_{n}\right\rangle$ and that, letting $X_{i}:=H+$ $\left\langle x_{1}, \ldots, x_{i}\right\rangle$ for $i=1, \ldots, n$ and $X_{0}:=H$, the subgroup $X_{i-1}$ is a proper subgroup of $X_{i}$ for $i=1, \ldots, n$. We shall prove by induction on $i=1, \ldots, n$, that

$$
H \in \mathfrak{C h a r}_{K}\left(X_{i}\right) .
$$

As $X=X_{n}$, this will give $H \in \mathfrak{C h a r}_{K}(X)$, as desired.

Before starting the induction, we note that according to Lemma 13(ii), all subgroups $X_{i}$, for $i=1, \ldots, n$, are auto-characterized, as each $X_{i-1}$ is open in $X_{i}$. For $i=1$, the assertion in condition (8) follows from Lemma 16. Assume that $1<i \leq n$ and condition (8) holds true for $i-1$, i.e., $H \in \mathfrak{C h a r}_{K}\left(X_{i-1}\right)$. Since $X_{i-1}$ is open in $X_{i}$, again Lemma 16 applied to $X_{i}=X_{i-1}+\left\langle x_{i}\right\rangle$ gives that $X_{i-1} \in \mathfrak{C h a r}_{K}\left(X_{i}\right)$. As $H \in \mathfrak{C h a r}_{K}\left(X_{i-1}\right)$ by our inductive hypothesis, we conclude with Corollary 2(i) that $H \in \mathfrak{C h a r}_{K}\left(X_{i}\right)$.

\subsection{Further Results on K-Characterized Subgroups}

The next corollary resolves an open question from [28]:

Corollary 9. Let $X$ be an infinite discrete abelian group and $H$ a subgroup of $X$. The following conditions are equivalent: 
(i) $H \in \mathfrak{C h a r}(X)$;

(ii) $H \in \mathfrak{C h a r}_{K}(X)$;

(iii) $[X: H] \leq \mathfrak{c}$.

Proof. (ii) $\Rightarrow$ (i) is clear, and (i) $\Rightarrow$ (iii) is Corollary 1.

(iii) $\Rightarrow$ (ii) If $[X: H]$ is infinite, then $H \in \mathfrak{C h a r}(X)$ by Theorem 7. Therefore, assume that $[X: H]$ is finite, then $H$ is infinite, and hence, $H$ is auto-characterized by Theorem 3; therefore, $H \in \mathfrak{C h a r}_{K}(X)$ by Theorem 8 .

We give now sufficient conditions for a non-closed characterized subgroup to be K-characterized.

Theorem 9. Let $X$ be a topological abelian group and $H \in \mathfrak{C h a r}(X)$ a non-closed subgroup of $X$, such that:

(i) $X / \bar{H}$ is $M A P$, and $(\widehat{X / \bar{H}}, \sigma(\widehat{X / \bar{H}}, X / \bar{H}))$ is separable;

(ii) if $1<[X: \bar{H}]<\omega$, then $\bar{H}$ is auto-characterized.

Then, $H \in \mathfrak{C h a r}_{K}(X)$.

Proof. As $H \neq \bar{H}$ is dense in $\bar{H}$ and obviously $H \in \mathfrak{C h a r}(\bar{H})$, we deduce that $H \in \mathfrak{C h a r}_{K}(\bar{H})$, as dense characterized subgroups are TB-characterized by Lemma 1(ii). If $\bar{H}=X$, we are done. Therefore, assume that $\bar{H}$ is proper.

Our aim now is to apply Corollary 2, so we need to check that $\bar{H} \in \mathfrak{C h a r}_{K}(X)$. If $\bar{H}$ has finite index in $X$, then $\bar{H}$ is auto-characterized by hypothesis, and so, Theorem 8 yields $\bar{H} \in \mathfrak{C h a r}_{K}(X)$. If $\bar{H}$ has infinite index in $X$, then $\bar{H} \in \mathfrak{C h a r}_{K}(X)$ by Theorem 6.

Corollary 10. Let $X$ be a divisible topological abelian group and $H \in \mathfrak{C h a r}(X)$ a non-closed subgroup of $X$, such that $\bar{H}$ is dually closed. If $(\widehat{X / \bar{H}}, \sigma(\widehat{X / \bar{H}}, X / \bar{H}))$ is separable, then $H \in \mathfrak{C h a r}_{K}(X)$. In particular, $H \in \mathfrak{C h a r}_{K}(X)$ whenever $\widehat{X / \bar{H}}$ is separable.

Proof. The first part of our hypothesis entails that $X / \bar{H}$ is MAP. Moreover, divisible topological abelian groups have no proper closed subgroup of finite index. Therefore, the first assertion follows directly from Theorem 9.

The topology $\sigma(\widehat{X / \bar{H}}, X / \bar{H})$ of the dual $\widehat{X / \bar{H}}$ is coarser than the compact-open topology of $\widehat{X / H}$, so that the separability of $\widehat{X / \bar{H}}$ yields the separability of $(\widehat{X / \bar{H}}, \sigma(\widehat{X / \bar{H}}, X / \bar{H}))$. Hence, the second assertion can be deduced from the first one.

In the case of connected locally compact abelian groups, one obtains the following stronger conclusion: 
Corollary 11. Let X be a connected locally compact abelian group. Then:

$$
\mathfrak{C h a r}_{K}(X)=\left\{\begin{array}{l}
\mathfrak{C h a r}(X), \text { if } X \text { is not compact } \\
\mathfrak{C h a r}(X) \backslash\{X\}, \text { if } X \text { is compact. }
\end{array}\right.
$$

Proof. The group $X$ is divisible, as connected locally compact abelian groups are divisible.

Let $H \in \mathfrak{C h a r}(X)$. Our next aim will be to check that $\widehat{X / \bar{H}}$ is separable. Indeed, if $H=s_{\mathbf{v}}(X)$ for $\mathbf{v} \in \widehat{X}^{\mathbb{N}}$, then one has the chain of subgroups $n_{\mathbf{v}}(X) \leq H \leq \bar{H} \leq X$. If $\bar{H}=X$, then $\widehat{X / \bar{H}}$ is trivially separable. Otherwise, $\bar{H}$ has infinite index in $X$, since $X / \bar{H}$ is divisible, so $X \widehat{/ n_{\mathbf{v}}(X)}$ is separable by Corollary 7; since $\bar{H}$ contains $n_{\mathbf{v}}(X)$, the quotient group $X / \bar{H}$ is a quotient group of $X / n_{\mathbf{v}}(X)$. Therefore, $\widehat{X / \bar{H}}$ is isomorphic to a subgroup of the separable group $X \widehat{/ n_{\mathbf{v}}(X)}$, so $\widehat{X / \bar{H}}$ is separable, as well (see [29]). Furthermore, $\bar{H}$ is dually closed (so $X / \bar{H}$ is MAP) by Fact 1.

If $H$ is not closed, Corollary 10 gives that $H \in \mathfrak{C h a r}_{K}(X)$. If $H$ is a proper closed subgroup of $X$, then $H$ has infinite index, as $X / H$ is divisible, so $H \in \mathfrak{C h a r}_{K}(X)$ by Theorem 6 . This proves the inclusion $\mathfrak{C h a r}(X) \backslash\{X\} \subseteq \mathfrak{C h a r}_{K}(X) \backslash\{X\}$, which, along with the obvious inclusion $\mathfrak{C h a r}_{K}(X) \subseteq \mathfrak{C h a r}(X)$, proves the equality $\mathfrak{C h a r}(X) \backslash$ $\{X\}=\mathfrak{C h a r}_{K}(X) \backslash\{X\}$.

It remains to consider the (closed) subgroup $H=X$, which obviously belongs to $\mathfrak{C h a r}(X)$. If $X$ is compact, then $X \notin \mathfrak{C h a r}_{K}(X)$, by Lemma 15, so $\mathfrak{C h a r}_{K}(X)=$ $\mathfrak{C h a r}(X) \backslash\{X\}$. If $X$ is not compact, then $H=X \in \mathfrak{C h a r}_{K}(X)$ by Theorem 3 and Remark 3(ii). Hence, $\mathfrak{C h a r}_{K}(X)=\mathfrak{C h a r}(X)$ in this case.

In particular, the above corollary yields $\mathfrak{C h a r}_{K}(\mathbb{T})=\mathfrak{C h a r}(\mathbb{T}) \backslash\{\mathbb{T}\}$ and $\mathfrak{C h a r}_{K}(\mathbb{R})=\mathfrak{C h a r}(\mathbb{R})$.

Remark 7. As we shall see in Corollary 18, connectedness is necessary in this corollary.

\section{N-Characterized Subgroups}

The following consequence of Lemma 14 gives a sufficient condition for a characterized subgroup to be $N$-characterized:

Corollary 12. Let $X$ be a topological abelian group and $H$ a subgroup of $X$, which is not auto-characterized. If $H \in \mathfrak{C h a r}(X)$, then $H \in \mathfrak{C h a r}_{N}(X)$.

Proof. Let $H=s_{\mathbf{v}}(X)$ for some $\mathbf{v} \in \widehat{X}^{\mathbb{N}}$. Then, $H \leq n_{\mathbf{v}_{(m)}}(X)$ for some $m \in \mathbb{N}$ by Lemma 14. Since $H=s_{\mathbf{v}}(X)=s_{\mathbf{v}_{(m)}}(X) \geq n_{\mathbf{v}_{(m)}}(X)$, we deduce that $H=$ $n_{\mathbf{v}_{(m)}}(X)$.

Here comes an easy criterion establishing when a subgroup is $N$-characterized. 
Theorem 10. Let $X$ be a topological abelian group and $H$ a subgroup of $X$. The following conditions are equivalent:

(i) $H$ is closed, and there exists a continuous injection $X / H \rightarrow \mathbb{T}^{\mathbb{N}}$;

(ii) $H \in \mathfrak{C h a r}_{N}(X)$;

(iii) $H$ is closed, and $\{0\}$ is $G_{\delta}$ in $(X / H)^{+}$.

Proof. (i) $\Rightarrow$ (ii) Suppose that there exists a continuous injection $j: X / H \rightarrow \mathbb{T}^{\mathbb{N}}$. Let $\pi: X \rightarrow X / H$ be the canonical projection. For every $n \in \mathbb{N}$, let $p_{n}: \mathbb{T}^{\mathbb{N}} \rightarrow \mathbb{T}$ be $n$-th projection, and let $v_{n}=p_{n} \circ j \circ \pi$.

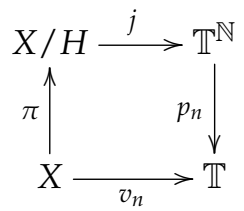

Therefore, $v_{n} \in \widehat{X}^{\mathbb{N}}$ for every $n \in \mathbb{N}$ and $H=n_{\mathbf{v}}(X)$, where $\mathbf{v}=\left(v_{n}\right)$.

(ii) $\Rightarrow$ (i) Let $H=n_{\mathbf{v}}(X)$ for $\mathbf{v} \in \widehat{X}^{\mathbb{N}}$. Let $\pi: X \rightarrow X / n_{\mathbf{v}}(X)$ be the canonical projection, and define $j: X / n_{\mathbf{v}}(X) \rightarrow \mathbb{T}^{\mathbb{N}}$ by $j(\pi(x))=\left(v_{n}(x)\right)_{n \in \mathbb{N}}$ for every $x \in$ $X$. Since $n_{\mathbf{v}}(X)=\bigcap_{n \in \mathbb{N}} \operatorname{ker} v_{n}$, then $j$ is well defined and injective. Moreover, $j$ is continuous.

Finally, (i) and (iii) are obviously equivalent.

The above criterion simplifies in the case of open subgroups:

Corollary 13. Let $X$ be a topological abelian group and $H$ an open subgroup of $X$. The following conditions are equivalent:

(i) $H \in \mathfrak{C h a r}(X)$;

(ii) $H \in \mathfrak{C h a r}_{N}(X)$;

(iii) $[X: H] \leq \mathfrak{c}$.

Proof. (ii) $\Rightarrow$ (i) is obvious, and (i) $\Rightarrow$ (iii) is Corollary 1.

(iii) $\Rightarrow$ (ii) Since $H$ is open, $X / H$ is discrete. By hypothesis $|X / H| \leq \mathfrak{c}$, so there exists a continuous injection $X / H \rightarrow \mathbb{T}^{\mathbb{N}}$. Hence, $H \in \mathfrak{C h a r}_{N}(X)$ by Theorem 10.

The next is another consequence of Theorem 10.

Corollary 14. Let $X$ be a metrizable precompact abelian group and $H$ a subgroup of $X$. The following conditions are equivalent:

(i) $H$ is closed;

(ii) $H$ is closed and $H \in \mathfrak{C h a r}(X)$; 
(iii) $H \in \mathfrak{C h a r}_{N}(X)$.

Proof. (iii) $\Rightarrow$ (ii) and (ii) $\Rightarrow$ (i) are clear.

(i) $\Rightarrow$ (iii) Since $X$ is metrizable, $X / H$ is metrizable, as well, and so, $\{0\}$ is $G_{\delta}$ in $X / H$. By Lemma 4, there exists a continuous injective homomorphism $X / H \rightarrow \mathbb{T}^{\mathbb{N}}$. Therefore, $H$ is $N$-characterized by Theorem 10 .

According to Theorem 6, one can add to the equivalent conditions in Corollaries 13 and 14 also " $H \in \mathfrak{C h a r}_{K}(X)$ ", in case $[X: H] \geq \omega$.

In the sequel, we consider the case of locally compact abelian groups. The following theorem was proven in Theorem B of [16] for compact abelian groups.

Theorem 11. Let $H$ be a locally compact abelian group and $H$ a subgroup of $X$. Then, $H \in \mathfrak{C h a r}(X)$ if and only if $H$ contains a closed $G_{\delta}$-subgroup $K$ of $X$, such that $H / K \in$ $\mathfrak{C h a r}(X / K)$, where $X / K$ is a metrizable locally compact abelian group.

Proof. The equivalence follows from Lemma 11, since the subgroup $n_{\mathbf{v}}(X)$ is closed and $G_{\delta}$ for every $\mathbf{v} \in \widehat{X}^{\mathbb{N}}$, by Lemma 7 (iv). Since $K$ is closed and $G_{\delta}, X / K$ is a metrizable locally compact abelian group.

By Lemma $7, n_{\mathbf{v}}(X)$ is always closed and characterized. Theorem 12 describes the closed characterized subgroups of the locally compact abelian groups $X$ by showing that these are precisely the $N$-characterized subgroups of $X$.

Theorem 12. Let $X$ be a locally compact abelian group and $H$ a subgroup of $X$. The following conditions are equivalent:

(i) $H$ is closed and $H \in \mathfrak{C h a r}(X)$;

(ii) $H \in \mathfrak{C h a r}_{N}(X)$;

(iii) $H$ is closed and $G_{\delta}$ in the Bohr topology;

(iv) $H$ is closed, $G_{\delta}$ and $[X: H] \leq \mathfrak{c}$.

(v) $H$ is closed, and $\widehat{X / H}$ is separable.

Proof. (iii) $\Rightarrow$ (ii) follows from Theorem 10 , (ii) $\Rightarrow$ (i) by Lemma 7 , and (ii) $\Leftrightarrow(v)$ is Corollary 7.

(iv) $\Rightarrow$ (iii) The group $X / H$ is locally compact, metrizable and has cardinality at most $\mathfrak{c}$; therefore, by Theorem 2 , there exists a continuous injective homomorphism $j: X / H \rightarrow \mathbb{T}^{\mathbb{N}}$. Then, Theorem 10 gives the thesis.

(i) $\Rightarrow$ (iv) Let $H=s_{\mathbf{v}}(X)$ for $\mathbf{v} \in \widehat{X}^{\mathbb{N}}$, and let $\pi: X \rightarrow X / n_{\mathbf{v}}(X)$ be the canonical projection. By Corollary $1,[X: H] \leq \mathfrak{c}$. By Lemma $7, n_{\mathbf{v}}(X) \leq s_{\mathbf{v}}(X)$, and $n_{\mathbf{v}}(X)$ is closed and $G_{\delta}$. Then, $X / n_{\mathbf{v}}(X)$ is a metrizable locally compact abelian group, and by hypothesis, $s_{\mathbf{v}}(X)$ is closed. Therefore, $s_{\mathbf{v}}(X) / n_{\mathbf{v}}(X)$ is closed and, hence, $G_{\delta}$ in $X / n_{\mathbf{v}}(X)$. Therefore, $s_{\mathbf{v}}(X)=\pi^{-1}\left(s_{\mathbf{v}}(X) / n_{\mathbf{v}}(X)\right)$ is closed and $G_{\delta}$ in $X$. 
We see now the following result from [16] as a consequence of Theorem 12.

Corollary 15. [16, Theorem A] Let $X$ be a compact abelian group and $H$ a closed subgroup of $X$. Then, $H \in \mathfrak{C h a r}(X)$ if and only if $H$ is $G_{\delta}$.

Proof. If $H$ is characterized, then $H$ is $G_{\delta}$ by Theorem 12 . Vice versa, assume that $H$ is $G_{\delta}$. Then, $X / H$ is compact and metrizable, hence $|X / H| \leq \mathfrak{c}$. Therefore, again Theorem 12 implies that $H$ is characterized.

In the following theorem, we use that the $G_{\delta}$-subgroups of a locally compact abelian group are always closed (see [28] (Theorem A.2.14)).

Theorem 13. Let $X$ be a compact abelian group and $H$ a subgroup of $X$. The following conditions are equivalent:

(i) $H \in \mathfrak{C h a r}_{K}(X)$ and $H$ is closed;

(ii) $H$ is $G_{\delta}$ and non-open;

(iii) $H \in \mathfrak{C h a r}_{N}(X)$ and $H$ is non-open;

(iv) $H \in \mathfrak{C h a r}(X)$ and $H$ is closed and non-open.

Proof. (i) $\Rightarrow$ (iv) Since Lemma 15 implies that $H$ is non-open, (iv) $\Leftrightarrow($ iii) by Theorem 12, and (iv) $\Leftrightarrow$ (ii) by Corollary 15 .

(ii) $\Rightarrow$ (i) Since $X / H$ is a metrizable compact non-discrete (hence infinite) abelian group, $\{0\}$ is closed and non-open in $X / H$; hence, $\{0\}$ is $K$-characterized in $X / H$ by Corollary 8. Therefore, $H$ is $K$-characterized by Proposition 2.

This theorem generalizes Corollary 15 as it implies that, for a closed non-open subgroup $H$ of a compact abelian group $X$, one has:

$$
H \text { is } G_{\delta} \Leftrightarrow H \in \mathfrak{C h a r}(X) \Leftrightarrow H \in \mathfrak{C h a r}_{N}(X) \Leftrightarrow H \in \mathfrak{C h a r}_{K}(X) .
$$

The following immediate consequence of Corollaries 13 and 9 shows that for a discrete abelian group all characterized subgroups are $\mathrm{N}$-characterized.

Corollary 16. Let $X$ be an infinite discrete abelian group and $H$ a subgroup of $X$. The following conditions are equivalent:

(i) $H \in \mathfrak{C h a r}(X)$;

(ii) $H \in \mathfrak{C h a r}_{N}(X)$;

(iii) $H \in \mathfrak{C h a r}_{K}(X)$;

(iv) $[X: H] \leq \mathfrak{c}$. 


\section{T-Characterized Closed Subgroups of Compact Abelian Groups}

In [30, Theorem 4], Gabriyelyan observed that if $\mathbf{u}$ is a $T$-sequence of an infinite countable abelian group $G$, then (see formula (1) for the definition of $\sigma_{\mathbf{v}}$ )

$$
n\left(G, \sigma_{\mathbf{v}}\right) \cong s_{\mathbf{v}}\left(\widehat{G_{d}}\right)^{\perp} \text { algebraically, }
$$

where $G_{d}$ denotes the abelian group $G$ endowed with the discrete topology. Therefore, the following fact is an immediate corollary of this result.

Fact 4. Let $\mathbf{v}$ be a T-sequence of an infinite countable abelian group $G$. Then:

(i) $\left(G, \sigma_{\mathbf{v}}\right)$ is MAP if and only if $\mathbf{v}$ is a TB-sequence;

(ii) $\left(G, \sigma_{\mathbf{v}}\right)$ is MinAP if and only if $s_{\mathbf{v}}\left(\widehat{G_{d}}\right)=\{0\}$ and $G=\langle\mathbf{v}\rangle$.

Recall that a topological abelian group $X$ is almost maximally almost periodic $(A M A P)$ if $n(X)$ is finite.

Remark 8. In relation to Fact 4, Lukács in [31] found a $T$-sequence in $\mathbb{Z}\left(p^{\infty}\right)$ that is not a $T B$-sequence, providing in this way an example of a non-trivial AMAP group. More precisely, he found a characterizing sequence $\mathbf{v}$ for $p^{m} \mathbb{J}_{p} \leq \mathbb{J}_{p}$ for a fixed $m \in \mathbb{N}_{+}$, i.e., $s_{\mathbf{v}}\left(\mathbb{J}_{p}\right)=p^{m} \mathbb{J}_{p}$. In this way, being $\mathbb{J}_{p} / p^{m} \mathbb{J}_{p}$ finite, then

$$
s_{\mathbf{v}}\left(\mathbb{J}_{p}\right)^{\perp}=n\left(\mathbb{Z}\left(p^{\infty}\right), \sigma_{\mathbf{v}}\right) \neq\{0\} \text { is finite. }
$$

Therefore, $\left(\mathbb{J}_{p}, \sigma_{\mathbf{v}}\right)$ is AMAP. Further results in this direction were obtained by Nguyen in [32]. Finally, Gabriyelyan in [33] proved that an abelian group $G$ admits an AMAP group topology if and only if $G$ has non-trivial torsion elements.

The following theorem, due to Gabriyelyan, links the notions of $T$-characterized subgroup and MinAP topology.

Theorem 14. [11] Let $X$ be a compact abelian group and $H$ a closed subgroup of $X$. Then, $H \in \mathfrak{C h a r}_{T}(X)$ if and only if $H$ is $G_{\delta}$ and $H^{\perp}$ carries a MinAP topology.

Following [4, §4], for a topological abelian group $X$ and a prime number $p$, we denote by $T_{p}(X)$ the closure of the subgroup $X_{p}=\left\{x \in X: p^{n} x \rightarrow 0\right\}$. In case $X$ is compact, one can prove that

$$
T_{p}(X)=\left\{m X: m \in \mathbb{N}_{+},(m, p)=1\right\} .
$$


In particular, $T_{p}(X)$ contains the connected component $c(X)$ of $X$. More precisely, if $X / c(X)=\prod_{p \in \mathbb{P}}\left(X(c(X))_{p}\right.$ is the topologically primary decomposition of the totally disconnected compact abelian group $X / c(X)$, then:

$$
T_{p}(X) / c(X) \cong(X / c(X))_{p}=T_{p}(X / c(X))
$$

Following [34], we say that $d \in \mathbb{N}$ is a proper divisor of $n \in \mathbb{N}$, provided that $d \notin\{0, n\}$ and $d m=n$ for some $m \in \mathbb{N}$. Note that, according to our definition, each $d \in \mathbb{N} \backslash\{0\}$ is a proper divisor of zero.

Definition 7. Let $G$ be an abelian group.

(i) For $n \in \mathbb{N}$ the group $G$ is said to be of exponent $n$ (denoted by $\exp (G)$ ) if $n G=\{0\}$, but $d G \neq\{0\}$ for every proper divisor $d$ of $n$. We say that $G$ is bounded if $\exp (G)>0$ and, otherwise, that $G$ is unbounded.

(ii) [35] If $G$ is bounded, the essential order eo $(G)$ of $G$ is the smallest $n \in \mathbb{N}_{+}$, such that $n G$ is finite. If $G$ is unbounded, we define $e o(G)=0$.

In the next theorem, we aim to give a detailed description of the closed characterized subgroups $H$ of $X$ that are not $T$-characterized. As stated in Corollary 15 , a closed subgroup $H$ of a compact abelian group $X$ is characterized if and only if $H$ is $G_{\delta}$ (i.e., $X / H$ is metrizable). This explains the blanket condition imposed on $H$ to be a $G_{\delta}$-subgroup of $X$.

Theorem 15. For a compact abelian group $X$ and a $G_{\delta}$-subgroup $H$ of $X$, the following conditions are equivalent:

(i) $H \notin \mathfrak{C h a r}_{T}(X)$;

(ii) $H^{\perp}$ does not admit a MinAP group topology;

(iii) there exists $m \in \mathbb{N}$, such that $m(X / H)$ is finite and non-trivial;

(iv) $\operatorname{eo}(X / H)<\exp (X / H)$;

(v) there exists a finite set $P$ of primes, so that:

(a) $T_{q}(X) \leq H$ for all $q \in \mathbb{P} \backslash P$,

(b) for every $p \in P$ there exist $k_{p} \in \mathbb{N}$ with $p^{k_{p}} T_{p}(X) \leq H$,

(c) there exists $p_{0} \in P$, such that $p_{0}^{k_{p_{0}}-1} T_{p_{0}}(X) \not L H$ and $p_{0}^{k_{p_{0}}-1} T_{p_{0}}(X) \cap H$ has finite index in $p_{0}^{k_{p_{0}}} T_{p_{0}}(X)$;

(vi) there exists a finite set $P$ of primes, so that $X / H \cong \prod_{p \in P} K_{p}$, where each $K_{p}$ is a compact p-group, and there exist some $p_{0} \in P$ and $k \in \mathbb{N}$, such that $p_{0}^{k} K_{p_{0}}$ is finite and non-trivial.

Proof. (i) $\Leftrightarrow$ (ii) is Theorem 14 , and (iii) $\Leftrightarrow$ (iv) is clear from the definition.

(ii) $\Leftrightarrow$ (iii) The main theorem in [36] states that an abelian group $G$ does not admit a MinAP group topology precisely when there exists $m \in \mathbb{N}_{+}$, such that $m G$ is finite 
and non-trivial. In our case, $G=H^{\perp}$ is topologically isomorphic to $\widehat{X / H}$, so $G$ does not admit a MinAP group topology if and only if $m \widehat{X / H}=m \widehat{(X / H)}$ is finite and non-trivial, and this occurs precisely when $m(X / H)$ is finite and non-trivial.

(vi) $\Rightarrow(\mathrm{v})$ Write $X / H \cong \prod_{p \in P} K_{p}$, where each $K_{p}$ is a compact $p$-group. Let $p^{k_{p}}=\exp \left(K_{p}\right)$ for every $p \in P$, and let $p_{0}^{k} K_{p_{0}}$ be finite and non-trivial for $p_{0} \in P$ and $k \in \mathbb{N}$.

Obviously, all $q \in \mathbb{P} \backslash P$ are coprime to $m=\exp (X / H)$. As $m X \leq H$, we deduce from the equality (9) that

$$
T_{q}(X) \leq H, \text { for all } q \in \mathbb{P} \backslash P .
$$

This proves (a). From (10), we deduce that that $c(X) \leq H$.

The quotient groups $X^{\prime}=X / c(X)$ and $H^{\prime}=H / c(X)$ are totally disconnected; hence, $X^{\prime}=\prod_{p \in \mathbb{P}} X_{p}^{\prime}$ and $H^{\prime}=\prod_{p \in \mathbb{P}} H_{p}^{\prime}$. Here,

$$
X_{p}^{\prime}=T_{p}(X) / c(X) \text { and } H_{p}^{\prime}=T_{p}(H) / c(X) \text { for every } p \in \mathbb{P} \text {. }
$$

Furthermore, $X^{\prime}=\prod_{p \in P} X_{p}^{\prime} \times \prod_{q \in \mathbb{P} \backslash P} X_{q}^{\prime}$. From (10), we deduce that $X_{q}^{\prime} \leq H_{q}^{\prime}$ for all $q \in \mathbb{P} \backslash P$. Therefore, $\prod_{q \in \mathbb{P} \backslash P} X_{q}^{\prime} \leq H^{\prime}$ and $H^{\prime}=\prod_{p \in P} H_{p}^{\prime} \times \prod_{q \in \mathbb{P} \backslash P} X_{q}^{\prime}$. Hence, $X / H=\prod_{p \in P} X_{p}^{\prime} / H_{p}^{\prime}$, and consequently, $X_{p}^{\prime} / H_{p}^{\prime} \cong K_{p}$ for all $p \in P$. Thus, $p^{k_{p}} X_{p}^{\prime} \leq H_{p}^{\prime}$ for all $p \in P$. Equivalently, $p^{k_{p}} T_{p}(X) \leq H$ for $p \in P$. This proves (b).

As $p_{0}^{k} K_{p_{0}}$ is finite and non-trivial, we deduce that $k<k_{p_{0}}$. Therefore, $p_{0}^{k_{p_{0}}-1} K_{p_{0}}$ is still finite and non-trivial. Hence, $p_{0}^{k_{p_{0}}-1} X_{p_{0}}^{\prime} \leq H_{p_{0}}^{\prime}$, and so,

$$
p_{0}^{k_{p_{0}}-1} T_{p_{0}}(X) \not \leq H .
$$

To prove the second assertion in (c), note that the finiteness of $p_{0}^{k} K_{p_{0}}$ yields that:

$$
p_{i}^{k_{p_{0}}-1}\left(X_{p_{0}}^{\prime} / H_{p_{0}}^{\prime}\right)=\left(p_{0}^{k_{p_{0}}-1} X_{p_{0}}^{\prime}+H_{p_{0}}^{\prime}\right) / H_{p_{0}}^{\prime} \cong p_{0}^{k_{p_{0}}-1} X_{p_{0}}^{\prime} /\left(H_{p_{0}}^{\prime} \cap p_{0}^{k_{p_{0}}-1} X_{p_{0}}^{\prime}\right)
$$

is finite. Hence, from (11), we deduce that $T_{p_{0}}(H) \cap p_{0}^{k_{p_{0}}-1} T_{p_{0}}(X)$ has finite index in $p_{0}^{k_{p_{0}}-1} T_{p_{0}}(X)$. Therefore,

$$
H \cap p_{0}^{k_{p_{0}}-1} T_{p_{0}}(X)=\cap T_{p_{0}}(H) \cap p_{0}^{k_{p_{0}}-1} T_{p_{0}}(X)
$$

has finite index in $p_{0}^{k_{p_{0}}-1} T_{p_{0}}(X)$.

(v) $\Rightarrow$ (iii) Let $m^{\prime}$ be the product of all $p^{k_{p}}$ when $p$ runs over $P$, and let $m=$ $m^{\prime} / p$. Then, an argument similar to the above argument shows that $m(X / H) \neq\{0\}$ is finite. 
Corollary 17. Let $X$ be a compact abelian group and $H$ a closed subgroup of $X$ that does not contain the connected component $c(X)$ of $X$. Then, $H \in \mathfrak{C h a r}_{T}(X)$ if and only if $H \in \mathfrak{C h a r}(X)$.

Proof. Clearly, $H$ T-characterized implies $H$ characterized. Therefore, assume that $H$ is characterized. Then, $H$ is $G_{\delta}$ in $X$ by Corollary 15. Let $\pi: X \rightarrow X / H$ be the canonical projection. Then, $\pi(c(G))$ is a non-trivial connected subgroup of $X / H$; hence, $X / H$ is unbounded, as its connected component $c(X / H)$ is a non-trivial divisible subgroup. According to Theorem 15, $H$ is $T$-characterized.

By Corollary 17, for a connected compact abelian group $X$ and $H$ a closed subgroup of $X$,

$$
H \in \mathfrak{C h a r} \mathfrak{r}_{T}(X) \Leftrightarrow H \in \mathfrak{C h a r}(X) \backslash\{X\} .
$$

This result was obtained in [11]; actually, the following more precise form holds (the equivalence $(\mathrm{i}) \Leftrightarrow(\mathrm{ii})$ is proven in [11, Theorem 1.14$])$ :

Corollary 18. For a compact abelian group $X$, the following conditions are equivalent:

(i) $X$ is connected;

(ii) $H \in \mathfrak{C h a r}_{T}(X) \Leftrightarrow H \in \mathfrak{C h a r}(X) \backslash\{X\}$ for every closed subgroup $H$ of $G$;

(iii) $H \in \mathfrak{C h a r}_{K}(X) \Leftrightarrow H \in \mathfrak{C h a r}(X) \backslash\{X\}$ for every closed subgroup $H$ of $G$.

Proof. (i) $\Rightarrow$ (ii) by (12), and (ii) $\Rightarrow$ (iii) is obvious.

(iii) $\Rightarrow$ (i) Assume that $X$ is not connected. Then, $X$ has a proper open subgroup $H$, as the connected component of $X$ is an intersection of clopen subgroups (see [22]). Then, $H \in \mathfrak{C h a r}(X) \backslash\{X\}$, but $H \notin \mathfrak{C} \mathfrak{h a r}{ }_{K}(X)$ by Lemma 15.

Obviously, each one of the above equivalent conditions implies that $H \in \mathfrak{C h a r}_{T}(X) \Leftrightarrow H \in \mathfrak{C h a r}_{K}(X)$ for every closed subgroup $H$ of a compact abelian group $X$. To see that in general the latter property is strictly weaker than $H \in \mathfrak{C h a r}_{T}(X)$, consider the group $X=\mathbb{Z}(3) \times \mathbb{Z}(2)^{\mathbb{N}}$. Then, for the closed subgroup $H=\{0\}$ of $X$, one has $H \in \mathfrak{C h a r}_{K}(X)$ by Theorem 13, while $H \notin \mathfrak{C h a r}_{T}(X)$ by Theorem 15 , as $3 X$ is finite and non-trivial (moreover, $\widehat{X}$ does not admit any MinAP group topology, as noticed by Remus; see [37]).

\section{Final Comments and Open Questions}

In this section, we collect various open questions arising throughout the paper.

For a topological abelian group $X$ and $\mathbf{v} \in \widehat{X}^{\mathbb{N}}$, we defined for each $m \in \mathbb{N}$ the closed subgroup $n_{\mathbf{v}_{(m)}}(X)$ in (4). Since these subgroups are contained one in each other, the increasing union $\mathbf{F}_{\mathbf{v}}(X):=\bigcup_{m \in \mathbb{N}} n_{\mathbf{v}_{(m)}}(X)$ is an $F_{\sigma}$-subgroup of $X$, and it is contained in $s_{\mathbf{v}}(X)$. We do not know whether $\mathbf{F}_{\mathbf{v}}(X)$ is characterized or not: 
Question 1. For a topological abelian group $X$ and $\mathbf{v} \in \widehat{X}^{\mathbb{N}}$, is $\mathbf{F}_{\mathbf{v}}(X)$ a characterized subgroup of $X$ ?

This question is motivated by [16, Theorem 1.11], where it is proven that under some additional restraint, the union of a countably infinite increasing chain of closed characterized subgroups of a metrizable compact abelian group is still characterized. On the other hand, it is known that every characterized subgroup is $F_{\sigma \delta}$ (see Lemma 5(iv)) and that the characterized subgroup need not be $F_{\sigma}$.

In analogy to $T$-characterized subgroups, we have introduced here the notion of the $T B$-characterized subgroup (see Definition 4). In relation to what is already known for $T$-characterized subgroups, one could consider the following general problem.

Problem 1. Study the TB-characterized subgroups of topological abelian groups.

Next comes a more precise question on the properties of $T$ - and $T B$-characterized subgroups. In fact, we do not know whether the counterpart of Corollary 2 is true for $T$ - and $T B$-characterized subgroups:

Question 2. Let $X$ be a topological abelian group and $X_{0}, X_{1}, X_{2}$ subgroups of $X$ with $X_{0} \leq X_{1} \leq X_{2}$, such that $X_{1}$ is dually embedded in $X_{2}$.

(i) If $X_{0} \in \mathfrak{C h a r}_{T}\left(X_{1}\right)$ and $X_{1} \in \mathfrak{C h a r} \mathfrak{r}_{T}\left(X_{2}\right)$, is then $X_{0} \in \mathfrak{C h a r} \mathfrak{r}_{T}\left(X_{2}\right)$ ?

(ii) If $X_{0} \in \mathfrak{C h a r}_{T B}\left(X_{1}\right)$ and $X_{1} \in \mathfrak{C h a r}_{T B}\left(X_{2}\right)$, is then $X_{0} \in \mathfrak{C h a r}_{T B}\left(X_{2}\right)$ ?

A full description of open $K$-characterized subgroups is given in Theorems 7 and 8 , while Theorem 6 describes the closed $K$-characterized subgroups of infinite index that are also $N$-characterized. Moreover, $N$-characterized closed subgroups of infinite index are $K$-characterized. This leaves open the following general problem and question.

Problem 2. For a topological abelian group $X$, describe $\mathfrak{C h a r}_{K}(X)$.

Question 3. Let $X$ be a topological abelian group. Can one add " $H \in \mathfrak{C h a r}_{K}(X)$ " as an equivalent condition in Theorem 6? Equivalently, does there exist a closed subgroup $H$ of $X$, such that $H \in \mathfrak{C h a r} \mathfrak{r}_{K}(X) \backslash \mathfrak{C h a r}{ }_{N}(X)$ ?

In Theorem 8, we have seen in particular that a proper open finite-index subgroup $H$ of a topological abelian group $X$ is auto-characterized precisely when $H \in \mathfrak{C h a r}_{K}(X)$. We do not know whether also the stronger condition $H \in \mathfrak{C h a r}_{T}(X)$ is equivalent: 
Question 4. Let $H$ be a topological abelian group and $H$ an open subgroup of $X$ of finite index. Does $H \in \mathfrak{C h a r}_{T}(X)$ whenever $H$ is auto-characterized? What about the case when $H$ is a topological direct summand of $X$ ?

By looking at Theorem 10 and Corollary 14, the following natural question arises:

Question 5. Are the closed $G_{\delta}$-subgroups of a precompact abelian groups always $N$-characterized?

This amounts to asking whether there exists a continuous injection from $X / F$ into $\mathbb{T}^{\mathbb{N}}$ for every closed $G_{\delta}$ subgroup $F$ of a precompact abelian group $X$; in other words, we are asking for a generalization of Lemma 4.

Acknowledgments: It is a pleasure to thank Wis Comfort for his useful comments related to (an equivalent form of) Question 5 and Saak Gabriyelyan for sending us his preprint [11] in 2012.

Anna Giordano Bruno is supported by Programma SIR 2014-MIUR (Project GADYGR, Number RBSI14V2LI, cup G22I15000160008). Dikran Dkranjan and Anna Giordano Bruno were partially supported by Fondazione Cassa di Risparmio di Padova e Rovigo (Progetto di Eccellenza "Algebraic structures and their applications").

Author Contributions: Dikran Dkranjan, Anna Giordano Bruno and Daniele Impieri contributed equally to this work.

Conflicts of Interest: The authors declare no conflict of interest.

\section{References}

1. Dikranjan, D.; Milan, C.; Tonolo, A. A Characterization of the Maximally Almost Periodic Abelian Groups. J. Pure Appl. Algebra 2005, 197, 23-41.

2. Armacost, D.L. The Structure of Locally Compact Abelian Groups; Marcel Dekker Inc.: New York, NY, USA, 1981; Volume 68.

3. Bíró, A.; Deshouillers, J.-M.; Sós, V.T. Good approximation and characterization of subgroups of $\mathbb{R} / \mathbb{Z}$. Studia Sci. Math. Hungar. 2001, 38, 97-113.

4. Dikranjan, D.; Prodanov, I.R.; Stoyanov, L.N. Topological Groups: Characters, Dualities and Minimal Group Topologies; Marcel Dekker Inc.: New York, NY, USA, 1990; Volume 130, p. 1.

5. Larcher, G. A convergence problem connected with continued fractions. Proc. Am. Math. Soc. 1988, 103, 718-722.

6. Petersen, K.; Shapiro, L. Induced flows. Trans. Am. Math. Soc. 1973, 177, 375-390.

7. Winkler, R. Ergodic group rotations, Hartman sets and Kronecker sequences. Monatsh. Math. 2002, 135, 333-343.

8. Gabriyelyan, S.S. Characterizable groups: Some results and open questions. Topol. Appl. 2012, 159, 2378-2391.

9. Dikranjan, D.; Impieri, D. Questions on the Borel complexity of characterized subgroups of compact abelian groups. Quest. Answ. Gen. Topol. 2014, 32, 127-144. 
10. Dikranjan, D.; Impieri, D. On the Borel complexity of characterized subgroups of compact abelian groups. Topol. Appl. 2015, in press.

11. Gabriyelyan, S.S. On T-characterized subgroups of compact abelian groups. Axioms 2015, 4, 194-212.

12. Negro, G. Polish LCA groups are strongly characterizable. Topol. Appl. 2014, 162, 66-75.

13. Dikranjan, D.; Kunen, K. Characterizing subgroups of compact abelian groups. J. Pure Appl. Algebra 2007, 208, 285-291.

14. Beiglböck, M.; Steineder, C.; Winkler, R. Sequences and filters of characters characterizing subgroups of compact abelian groups. Topol. Appl. 2006, 153, 1682-1695.

15. Dikranjan, D. Closure operators in topological groups related to von Neumann's kernel. Topol. Appl. 2006, 153, 1930-1955.

16. Dikranjan, D.; Gabriyelyan, S.S. On characterized subgroups of compact abelian groups. Topol. Appl. 2013, 160, 2427-2442.

17. Borel, J.P. Sous-groupes de $\mathbf{R}$ liés à la répartition modulo 1 de suites. Ann. Fac. Sci. Toulouse Math. 1983, 5, 217-235.

18. Gabriyelyan, S.S. On characterized subgroups of Abelian topological groups $X$ and the group of all X-valued null sequences. Comment. Math. Univ. Carolin. 2014, 55, 73-99.

19. Hart, J.E.; Kunen, K. Limits in function spaces and compact groups. Topol. Appl. 2005, 151, 157-168.

20. Hart, J.E.; Kunen, K. Limits in compact abelian groups. Topol. Appl. 2006, 153, 991-1002.

21. Comfort, W.W.; Ross, K.A. Topologies induced by groups of characters. Fund. Math. 1964, 55, 283-291.

22. Hewitt, E.; Ross, K.A. Abstract Harmonic Analysis, 2nd ed.; Springer-Verlag: Berlin, Germany, 1979; Volume 115.

23. Comfort, W.W.; Trigos-Arrieta, F.J.; Wu, T.S. The Bohr compactification, modulo a metrizable subgroup. Fund. Math. 1993, 143, 119-136.

24. Dikranjan, D.; Giordano Bruno, A. TB-sequences in abelian topological groups. 2015, in preparation.

25. Efimov, B.A. Mappings and imbeddings of dyadic spaces. Mat. Sb. (N.S.) 1977, 103, 52-68.

26. Dikranjan, D.; Tholen, W. Categorical Structure of Closure Operators; Kluwer Academic Publishers Group: Dordrecht, The Netherlands, 1995; Volume 346.

27. Barbieri, G.; Dikranjan, D.; Milan, C.; Weber, H. Answer to Raczkowski's questions on convergent sequences of integers. Topol. Appl. 2003, 132, 89-101.

28. Impieri, D. Characterized Subgroups. Ph.D. Thesis, Università degli studi di Udine, Udine, Italy, July 2015.

29. Comfort, W.W.; Itzkowitz, G.L. Density character in topological groups. Math. Ann. 1977, 226, 223-227.

30. Gabriyelyan, S.S. On T-sequences and characterized subgroups. Topol. Appl. 2010, 157, 2834-2843.

31. Lukács, G. Almost maximally almost-periodic group topologies determined by T-sequences. Topol. Appl. 2006, 153, 2922-2932. 
32. Nguyen, A.P. Which infinite abelian groups admit an almost maximally almost-periodic group topology? Topol. Appl. 2009, 156, 2642-2650.

33. Gabriyelyan, S.S. Characterization of almost maximally almost-periodic groups. Topol. Appl. 2009, 156, 2214-2219.

34. Dikranjan, D.; Shakhmatov, D. The Markov-Zariski topology of an abelian group. J. Algebra 2010, 324, 1125-1158.

35. Givens, B.N.; Kunen, K. Chromatic numbers and Bohr topologies. Topol. Appl. 2003, 131, 189-202.

36. Dikranjan, D.; Shakhmatov, D. Final solution of Protasov-Comfort's problem on minimally almost periodic group topologies. 2014, arXiv:1410.3313.

37. Comfort, W.W. Problems on topological groups and other homogeneous spaces. Available online: http:/ / citeseerx.ist.psu.edu/viewdoc/summary?doi=10.1.1.154.3370 (accessed on 9 October 2015). 


\section{Locally Quasi-Convex Compatible Topologies on a Topological Group}

\section{Lydia Außenhofer, Dikran Dikranjan and Elena Martín-Peinador}

Abstract: For a locally quasi-convex topological abelian group $(G, \tau)$, we study the poset $\mathscr{C}(G, \tau)$ of all locally quasi-convex topologies on $G$ that are compatible with $\tau$ (i.e., have the same dual as $(G, \tau)$ ) ordered by inclusion. Obviously, this poset has always a bottom element, namely the weak topology $\sigma(G, \widehat{G})$. Whether it has also a top element is an open question. We study both quantitative aspects of this poset (its size) and its qualitative aspects, e.g., its chains and anti-chains. Since we are mostly interested in estimates "from below", our strategy consists of finding appropriate subgroups $H$ of $G$ that are easier to handle and show that $\mathscr{C}(H)$ and $\mathscr{C}(G / H)$ are large and embed, as a poset, in $\mathscr{C}(G, \tau)$. Important special results are: (i) if $K$ is a compact subgroup of a locally quasi-convex group $G$, then $\mathscr{C}(G)$ and $\mathscr{C}(G / K)$ are quasi-isomorphic (3.15); (ii) if $D$ is a discrete abelian group of infinite rank, then $\mathscr{C}(D)$ is quasi-isomorphic to the poset $\mathfrak{F}_{D}$ of filters on $D$ (4.5). Combining both results, we prove that for an LCA (locally compact abelian) group $G$ with an open subgroup of infinite co-rank (this class includes, among others, all non- $\sigma$-compact LCA groups), the poset $\mathscr{C}(G)$ is as big as the underlying topological structure of $(G, \tau)$ (and set theory) allows. For a metrizable connected compact group $X$, the group of null sequences $G=c_{0}(X)$ with the topology of uniform convergence is studied. We prove that $\mathscr{C}(G)$ is quasi-isomorphic to $\mathscr{P}(\mathbb{R})(6.9)$.

Reprinted from Axioms. Cite as: Außenhofer, L.; Dikranjan, D.; Martín-Peinador, E. Locally Quasi-Convex Compatible Topologies on a Topological Group. Axioms 2016, 4, 436-458.

\section{Introduction}

All groups in this paper are abelian, and for a group $G$, we denote by $\mathcal{L}(G)$ (resp., $\mathcal{T}(G)$ ) the set of all (Hausdorff) group topologies on $G$.

Varopoulos posed the question of the description of the group topologies on an abelian group $G$ having a given character group $H$ and called them compatible topologies for the duality $(G, H)[1]$. As the author explains, the question is motivated by Mackey's theorem, which holds in the framework of locally convex spaces. He treated the question within the class of locally precompact abelian groups. Later on, this problem was set in a bigger generality in [2]; namely, within the class of locally quasi-convex groups. This is a class of abelian topological groups, which properly contains the class of locally convex spaces, a fact that makes the attempt to generalize the Mackey-Arens Theorem more natural. We denote by $\mathscr{C}(G, \tau)$, or simply by $\mathscr{C}(G)$ 
if no misunderstanding can arise, the set of all locally quasi-convex group topologies on $G$, which are compatible for $G^{\wedge}$, the character group of $G$.

The bottom element of $\mathscr{C}(G, \tau)$ is the weak topology $\sigma\left(G, G^{\wedge}\right)$. In [2], it was asked if the poset $\mathscr{C}(G, \tau)$ has a top element. We denote the supremum (in $\mathcal{L}(G)$ ) of $\mathscr{C}(G, \tau)$ by $S(G, \tau)$. Then, $S(G, \tau)$ is a locally quasi-convex topology; nevertheless, the question of whether $S(G, \tau) \in \mathscr{C}(G, \tau)$ is still open. In case $S(G, \tau) \in \mathscr{C}(G, \tau)$, it is called Mackey topology and denoted by $\mu\left(G, G^{\wedge}\right)$. Furthermore, if $\tau=\mu\left(G, G^{\wedge}\right)$, the group $(G, \tau)$ is called a Mackey group. This is a generalization of the notion of a Mackey locally convex space.

Theorem 1.1. ([2]) If a locally quasi-convex group $G$ is Čech complete (in particular, complete metrizable or locally compact abelian (LCA)), then $G$ is a Mackey group.

In particular, one has the following immediate corollary concerning the special case when $|\mathscr{C}(G, \tau)|=1$ (i.e., $\left.\tau=\sigma\left(G, G^{\wedge}\right)=\mu\left(G, G^{\wedge}\right)\right)$ :

Corollary 1.2. If $G$ is an $L C A$ group, then $|\mathscr{C}(G)|=1$ if and only if $G$ is compact.

Further attention to topological groups $G$ with $|\mathscr{C}(G)|=1$ is paid in [3,4], where many examples are given, inspired by [5] (in particular, it is proven that this equality holds for pseudocompact abelian groups).

This paper offers a solution for the following questions from [3] in the case that $G$ is a non- $\sigma$-compact LCA group:

Questions 1.3. Let $G$ be a locally quasi-convex topological group.

(a) [3] (Question 8.92) Compute the cardinality of the poset $\mathscr{C}(G)$.

(b) [3] (Problem 8.93) Under which conditions on the group $G$ is the poset $\mathscr{C}(G)$ a chain?

More precisely, in the light of Corollary 1.2, we show that if an LCA group is sufficiently far from being compact (e.g., non- $\sigma$-compact), then the poset $\mathscr{C}(G)$ is as big (and as far from being a chain) as possible (see Section 1.2 for details).

\subsection{Measuring Posets of Group Topologies}

In order to face Question 1.3, one needs a tool to measure the poset $\mathscr{C}(G)$ of group topologies.

The complete lattice $\mathcal{L}(G)$ for a group $G$ and some of its subsets (e.g., $\mathcal{T}(G)$, the subset $\mathcal{B}(G)$ of precompact topologies, its subset $\mathcal{P}_{S C}(G)$ of pseudocompact topologies, etc.) have been studied by many authors [6-11]. In particular, many cardinal invariants of the specific subposets of $\mathcal{T}(G)$ have been computed by using the simple idea of replacing the complicated posets $\mathcal{T}(G), \mathcal{B}(G), \mathcal{P}_{S C}(G)$, etc., by some naturally-defined simple posets of purely combinatorial nature (e.g., the powerset $\mathscr{P}(G)$ ordered by inclusion). Since $\mathscr{C}(G) \cap \mathcal{B}(G)=\{\sigma(G, \widehat{G})\}$ 
is a singleton for a locally quasi-convex group $G$, we cannot make use of the above-mentioned results where $\mathcal{B}(G)$ (and even $\mathcal{P}_{s c}(G)$ ) were shown to be as big as possible.

Let us recall that a subset $A$ of a partially-ordered set $X$ is called anti-chain if its members are pairwise incomparable. The maximal size of an anti-chain in a partially-ordered set $X$ is called the width of $X$ and denoted by width $(X)$.

In order to measure the size and width of the poset $\mathscr{C}(G)$, we introduce the following notion:

Definition 1.4. Two posets $(X, \leq)$ and $(Y, \leq)$ are:

- isomorphic (we write $X \cong Y$ ) if there exists a poset isomorphism $X \longrightarrow Y$;

- ([9-11]) quasi-isomorphic (we write $X \stackrel{\cong}{\cong}$ ) if there exist poset embeddings:

$$
(X, \leq) \hookrightarrow(Y, \leq) \quad \text { and } \quad(Y, \leq) \hookrightarrow(X, \leq)
$$

Clearly, quasi-isomorphic posets share the same monotone cardinal invariants, e.g., cardinality, width, maximum size of chains, etc. As a "sample" poset of combinatorial nature will be used, the poset is defined in the following example.

Example 1.5. Let $X$ be an infinite set, and let Fil $_{X}$ be the set of all free filters (i.e., filters $\mathscr{F}$ on $X$ with $\cap \mathscr{F}=\varnothing$ ) ordered by inclusion. The bottom element $\varphi_{0}$ of Fil $_{X}$ is known as the Fréchet filter; its elements are the complements of finite sets. A filter on $X$ is free iff it contains $\varphi_{0}$. For the sake of completeness, we shall add to Fil $X_{X}$ also the power set $\mathscr{P}(X)$ of $X$ to obtain the complete lattice:

$$
\mathfrak{F}_{X}:=\operatorname{Fil}_{X} \cup\{\mathscr{P}(X)\} \subseteq \mathscr{P}(\mathscr{P}(X))
$$

having as top element $\mathscr{P}(X)$ and bottom element $\varphi_{0}$. We shall denote $\mathfrak{F}_{X}$ also by $\mathfrak{F}_{\kappa}$ where $\kappa=|X|$, if we do not need to indicate the specific set $X$ and only care about its size $\kappa$. Then:

$$
\operatorname{width}\left(\mathfrak{F}_{X}\right)=\left|\mathfrak{F}_{X}\right|=2^{2^{|X|}}
$$

since there are $2^{2^{|X|}}$ ultrafilters on $X([12])$ that obviously form an anti-chain; the reverse inequalities width $\left(\mathfrak{F}_{X}\right) \leq\left|\mathfrak{F}_{X}\right| \leq 2^{2^{|X|}}$ are obvious, as $\mathfrak{F}_{X}$ is contained in the power set $\mathscr{P}(\mathscr{P}(X))$ of $\mathscr{P}(X)$ having size $2^{2^{|X|}}$.

Our choice of $\mathfrak{F}_{X}$ as a sample poset is justified by the following simple fact: Proposition 1.6. For every infinite group $G$, there exists a poset embedding $\mathcal{T}(G) \rightarrow \mathfrak{F}_{|G|}$. Proof. Every Hausdorff group topology $\tau$ on $G$ is completely determined by the filter $\mathcal{N}_{G, \tau}$ of all $\tau$-neighborhoods of zero. Since $\tau$ is Hausdorff, zero is the only common point of all members of $\mathcal{N}_{G, \tau}$. Hence, by restricting this filter to the set $X=G \backslash\{0\}$, 
we obtain an element of $\mathfrak{F}_{X}$. In other words, we defined an injective monotone map $\mathcal{T}(G) \rightarrow \mathfrak{F}_{X}$. Note that the discrete topology on $X$ is mapped to $\mathscr{P}(X)$.

Let us recall that a poset $(X, \leq)$ is called a dcpo (directedly complete poset) if all directed suprema in $X$ exist ([13] (Definition 2.1.13)). Clearly, a dcpo with a bottom element is a complete lattice precisely when it is a lattice. The relevant fact that the poset $\mathscr{C}(G, \tau)$ is a dcpo was established in [2] (Proposition 1.14):

Fact 1.7. [2] $\mathscr{C}(G, \tau)$ is a dcpo, i.e., stable under directed suprema taken in the complete lattice $\mathcal{L}(G)$.

According to the above fact, the poset $\mathscr{C}(G)$ has maximal elements (actually, every element of $\mathscr{C}(G)$ is contained in a maximal element of $\mathscr{C}(G)$ ), so it has a top element precisely when it is a lattice. In such a case, $\mathscr{C}(G)$ is a complete lattice, and $G$ has a Mackey topology.

\subsection{Main Results}

We give for a large class of LCA groups concrete descriptions of the set of compatible locally quasi-convex group topologies. More precisely, we first establish the following.

(a) If $G$ is a locally quasi-convex abelian group and $K$ is a compact subgroup of $G$, then $\mathscr{C}(G) \cong \mathscr{C}(G / K)$ (Theorem 3.15).

(b) If $H$ is an open subgroup of $G$, then there exist poset embeddings $\mathscr{C}(H) \stackrel{\Psi}{\hookrightarrow}$ $\mathscr{C}(G) \stackrel{\Theta}{\hookleftarrow} \mathscr{C}(G / H)$ (Theorem 3.6 and Corollary 3.11).

(c) For every discrete group $D$ of infinite rank, the set $\mathscr{C}(D)$ is quasi-isomorphic to the set of filters on $D$ (Lemma 4.5).

Item (a) above gives a precise and useful form of the intuitive understanding (based on Corollary 1.2) that compact subgroups are "negligible" when $\mathscr{C}(G)$ is computed for a locally quasi-convex group $G$. In particular, this holds for an LCA group $G$ and a compact subgroup $K$. This allows us to reduce the computation of $\mathscr{C}(G)$ to the case of much simpler (e.g., discrete) groups $G$.

Items (b) and (c) yield the following:

Theorem A. If $H$ is an open subgroup of a locally quasi-convex group $(G, \mathcal{T})$ with $r(G / H) \geq \omega$, then there is a poset embedding:

$$
\mathfrak{F}_{|G / H|} \hookrightarrow \mathscr{C}(G)
$$

so that $|\mathscr{C}(G)| \geq \operatorname{width}(\mathscr{C}(G)) \geq 2^{2^{|G / H|}}$. 
Corollary 1.8. If a locally quasi-convex group $(G, \mathcal{T})$ has an open subgroup of infinite co-rank, then:

$$
|\mathscr{C}(G)| \geq \operatorname{width}(\mathscr{C}(G)) \geq 2^{\mathfrak{c}}
$$

so $\mathscr{C}(G)$ is not a chain.

This answers Problem 8.93 of [3] (i.e., Question 1.3(b)) in the case of groups that have a discrete quotient of infinite rank.

Item (b) and Theorem A suggest considering open subgroups $H$ of $G$ with the highest possible co-rank. Motivated by this, we introduce the following notion.

Definition 1.9. A topological abelian group $G$ is called $r$-disconnected if $G$ has an open subgroup $\mathrm{H}$ of infinite co-rank.

For example, a non- $\sigma$-compact LCA group is $r$-disconnected (see Section 2 for a proof, as well as for further details). According to (2), r-disconnected groups $G$ have a sufficiently large poset $\mathscr{C}(G)$. The term $r$-disconnected is suggested by the fact that an $r$-disconnected group $G$ has open subgroups $H$ of infinite co-rank, so its degree of disconnectedness of $G$ "is measured" by the rank of $G / H$. Now, we introduce a cardinal invariant to carry out the measuring of $r$-disconnectedness in a natural way:

Definition 1.10. For a topological group $G$ define the discrete rank (d-rank) of $G$ by:

$$
\varrho(G)=\sup \{r(G / H): H \text { open subgroup of } G\}
$$

Clearly, r-disconnected groups $G$ have infinite $\varrho(G)$, but a group with $\varrho(G)=\omega$ need not be r-disconnected unless $G$ is LCA. For the properties of this cardinal invariant and its connection to the compact covering number $k(G)$, see Section 2.

In the case of LCA groups, it is possible to provide an embedding in the opposite direction of the embedding (2). In fact, we prove the following theorem (see Section 5 for the proof of Theorems A and B).

Theorem B. For every $r$-disconnected LCA group $G$, there exist poset embeddings:

$$
\mathfrak{F}_{\varrho(G)} \hookrightarrow \mathscr{C}(G) \hookrightarrow \mathfrak{F}_{\mathfrak{c} \cdot \varrho(G)}
$$

in particular,

$$
2^{2^{\rho(G)}} \leq|\mathscr{C}(G)| \leq 2^{2^{\cdot \cdot e(G)}} \text { and } 2^{2^{\rho(G)}} \leq \operatorname{width}(\mathscr{C}(G)) \leq 2^{2^{c \cdot e(G)}}
$$

If $G$ is totally disconnected, then $\mathscr{C}(G) \stackrel{q . i .}{\cong} \mathfrak{F}_{Q(G)}$.

It turns out that the inclusions (4) hold also under a slightly stronger condition of purely topological flavor 
Corollary C. Let $G$ be a non $\sigma$-compact LCA group, then $\varrho(G)>\omega$; so, the inclusions (4) hold.

The proof of this corollary will be given in Section 5 .

Since both sides of the concluding inequality in Theorem B coincide when $2^{\varrho(G)} \geq 2^{\mathfrak{c}}$, we obtain the equality $|\mathscr{C}(G)|=2^{2^{\varrho(G)}}$. One can say something more precise under a stronger assumption:

Corollary 1.11. If an $L C A$ group $G$ has $\varrho(G) \geq \mathfrak{c}$, then $\mathscr{C}(G) \stackrel{\text { q.i. }}{\cong} \mathfrak{F}_{\varrho(G)}$.

Since $\varrho(G) \geq \mathfrak{c}$ implies that the group $G$ is $r$-disconnected, Theorem B applies. Now, the assertion follows from the inclusions (4).

From Corollary C and Corollary 1.11, one can immediately deduce:

Corollary 1.12. Under the assumption of $C H, \mathscr{C}(G) \stackrel{\text { q.i. }}{\cong} \mathfrak{F}_{Q(G)}$ holds for every non-б-compact LCA group.

The last section is dedicated to a natural generalization of the metrizable LCA groups, namely the complete metrizable locally quasi-convex (so, necessarily Mackey) groups. In Theorem 6.1, we prove that for every non-trivial compact connected metrizable group $X$, the group $c_{0}(X)$ of null sequences in $X$ carries a Polish Mackey topology $\tau$, and $\mathscr{C}(G)$ is quasi-isomorphic to $\mathscr{P}(\mathbb{N})$, so it contains exactly $\mathfrak{c}$ many connected separable metrizable locally quasi-convex non-Mackey topologies compatible with $\mathfrak{p}_{0}$, the topology induced by the product topology $c_{0}(X) \hookrightarrow X^{\mathbb{N}}$.

The paper is organized as follows. Properties of the $d$-rank and its connection to the compact covering number are exposed in Section 2. In Section 3, we give general properties of the compatible topologies and of the poset $\mathscr{C}(G)$ (mainly invariance properties w.r.t. passage to products, subgroups and quotient groups). They enable us to prove in Section 4 that $\mathscr{C}(G) \stackrel{q . i .}{\cong} \mathfrak{F}_{|G|}$ for every discrete group $G$ of infinite rank. Using this fact, we prove Theorems A and B and Corollary $C$ in Section 5. Finally, in Section 6, we investigate $\mathscr{C}(G)$ for $G=c_{0}(X)$, where $X$ is a non-trivial, compact connected metrizable group, and we show that $\mathscr{C}(G)$ is quasi-isomorphic to $\mathscr{P}(\mathbb{R})$. In Section 7, we collect final remarks and open questions.

The main results of the paper were exposed in talks of the second named author at the Prague TopoSym 2011 and at the Seventh Italian Spanish Conference on General Topology in Badajoz in September 2010, as well as at a Colloquim talk at Complutense University of Madrid in 2010.

\section{Notation and preliminaries.}

We denote by $\mathbb{N}=\{0,1,2, \ldots\}$ the natural numbers, by $\mathbb{P}$ the prime numbers, by $\mathbb{Z}$ the group of integers, by $\mathbb{Q}$ the group of rational numbers, by $\mathbb{R}$ the group of 
real numbers and by $\mathbb{Z}_{m}$ the cyclic group of order $m$. Let $\mathbb{T}$ denote the quotient group $\mathbb{R} / \mathbb{Z}$. We shall identify it with the interval $(-1 / 2,1 / 2]$ with addition modulo one. It is isomorphic to the unit circle in the complex plane, with the ordinary product of complex numbers. Let $\mathbb{T}_{+}=:[-1 / 4,1 / 4] \subseteq \mathbb{T}$. For a topological abelian group $G$, the character group or dual group $G^{\wedge}$ is the set of all continuous homomorphisms from $G$ to $\mathbb{T}$, with addition defined pointwise.

For an abelian topological group $(G, \tau)$ and its dual $G^{\wedge}$, we shall denote the weak topology $\sigma\left(G, G^{\wedge}\right)$ also by $\tau^{+}$. An abelian topological group $(G, \tau)$ is said to be maximally almost periodic (MAP), if the continuous characters of $G$ separate the points of $G$ (i.e., if $\tau^{+}$is Hausdorff).

In the beginning of the 1950s, Vilenkin defined the notions of locally quasi-convex subsets and locally quasi-convex groups for abelian Hausdorff groups. These settings generalize the terms convexity and local convexity in topological vector spaces. A subset $M$ of an abelian topological group $G$ is said to be quasi-convex if every element in $G \backslash M$ can be separated from $M$ by means of a continuous character. More precisely: for any $z \notin M$, there exists $\xi \in G^{\wedge}$, such that $\xi(M) \subseteq \mathbb{T}_{+}$and $\xi(z) \notin \mathbb{T}_{+}$. An abelian topological group is called locally quasi-convex if it has a neighborhood basis of zero consisting of quasi-convex sets. The most prominent examples of locally quasi-convex groups are $\mathbb{T}, \mathbb{R}$ and discrete groups and, more generally, locally compact abelian groups and locally convex vector spaces (see [14] for an account of the properties of quasi-convex groups).

For an abelian group $G$, we shall denote by $\mathcal{L Q C}(G)$ the poset of all Hausdorff locally quasi-convex group topologies on $G$. Then, $\mathcal{B}(G) \subseteq \mathcal{L} \mathcal{Q C}(G) \subseteq \mathcal{T}(G)$.

We use frequently the fact that the supremum of locally quasi-convex topologies is again locally quasi-convex.

Consider the mapping:

$$
\mathcal{L Q C}(G) \longrightarrow \operatorname{Hom}(G, \mathbb{T}), \tau \longmapsto(G, \tau)^{\wedge}
$$

Its restriction to the set $\mathcal{B}(G)$ of all precompact topologies is injective. The image of this mapping is the set of all dense subgroups of the dual group of the discrete group $G$, and the fiber of $(G, \tau)^{\wedge}$ is precisely $\mathscr{C}(G, \tau)$. Therefore, $\{\mathscr{C}(G, \tau)$ : $\tau \in \mathcal{L} \mathcal{Q C}(G)\}$ forms a partition of $\mathcal{L Q C}(G)$. Since $(G, \tau)^{\wedge}=\left(G, \tau^{+}\right)^{\wedge}$ and $\tau^{+}$is a precompact, we obtain:

$$
\mathcal{L} \mathcal{Q C}(G)=\bigcup_{\tau \in \mathcal{L} \mathcal{Q C}(G)} \mathscr{C}(G, \tau)=\bigcup_{\tau \in \mathcal{L} \mathcal{Q C}(G)} \mathscr{C}\left(G, \tau^{+}\right)=\biguplus_{\tau \in \mathcal{B}(G)} \mathscr{C}(G, \tau)
$$

One of the aims of this paper is to show that each member of the partition (5) containing a non- $\sigma$-compact LCA group topology has the same size as the whole $\mathcal{L} \mathcal{Q}(G)$ and actually the same size as $\mathcal{T}(G)$. 
For an abelian group $G$, we denote by $r_{0}(G)$ the free-rank of $G$, and for a prime $p$, we denote by $r_{p}(G)$ the $p$-rank of $G$ (namely, $\operatorname{dim}_{\mathbb{Z}_{p}}\{x \in G: p x=0\}$ ). Finally, the rank of $G$ is defined by:

$$
r(G)=r_{0}(G)+\sup \left\{r_{p}(G): p \in \mathbb{P}\right\}
$$

For a subgroup $H$ of $G$, the co-rank of $H$ in $G$ is defined to be the $\operatorname{rank} r(G / H)$ of the quotient group.

\section{The Connections between the Compact Covering Number and the $d$-Rank of Topological Groups}

For a topological group $G$, we denote by $k(G)$ the compact covering number of $G$, i.e., the minimum number of compact sets whose union covers $G$. Clearly, either $k(G)=1$ (precisely when $G$ is compact) or $k(G) \geq \omega$. If $k(G) \leq \omega$, the group is called $\sigma$-compact. We shall see below (Theorem 2.7) that there is a close connection between the cardinal invariants $k(G)$ and $\varrho(G)$.

Clearly, $\varrho(G)=0$ precisely when $G$ has no proper open subgroups. This class of groups (in different, but equivalent terms) was introduced by Enflo [15] under the name locally generated groups (connected groups are obviously the leading example of locally generated groups). Therefore, the non-r-disconnected groups $G$ (in particular, the groups with $\varrho(G)<\omega$ ) can be considered as a natural generalization of the locally generated groups introduced by Enflo.

Furthermore, $\varrho(G) \leq \omega$ when $G$ is either $\sigma$-compact or separable, since both conditions imply that all discrete quotients of $G$ are countable (see Theorem 2.7 for the connection between $\sigma$-compactness and countability of the $d$-rank $\varrho$ ).

As the next example shows, the supremum in Equation (3) need not be attained by any specific open subgroup of $G$. We shall see in Theorem 2.7 that for $r$-disconnected LCA groups, this supremum is always a maximum.

Example 2.1. Consider the group $G=\bigoplus_{n} G_{n}$, where $G_{n}=\bigoplus_{\aleph_{n}} \mathbb{Q}$ is discrete and $G$ carries the product topology. A base of neighborhoods of zero is formed by the open subgroups $W_{m}=\bigoplus_{n>m} G_{n}$, so that $G$ is $r$-disconnected as $r\left(G / W_{m}\right)=\aleph_{m}$ for every $m \in \mathbb{N}$. Moreover, $\varrho(G)=|G|=\aleph_{\omega}$. On the other hand, every open subgroup $H$ of $G$ contains some $W_{m}$, so $r(G / H) \leq r\left(G / W_{m}\right)=\aleph_{m}<\varrho(G)$.

Generalizing the well-known fact that connected locally compact groups are $\sigma$-compact, we characterize below the LCA groups that are not r-disconnected, showing that they are $\sigma$-compact of a very special form (see Example 2.6). They will be the object of study in [16], whereas in this paper, we are interested in $r$-disconnected groups that may well be $\sigma$-compact (an example to this effect is any discrete countable group of infinite rank).

Let us start with the description of the groups of finite rank. 
Fact 2.2. Let $G$ be an infinite abelian group. Then, the following statements are equivalent:

(a) $r(G)<\infty$;

(b) $G$ is isomorphic to a subgroup of a group of the form $\mathbb{Q}^{m} \times \prod_{i=1}^{k} \mathbb{Z}\left(p_{i}^{\infty}\right)$, where $m, k \in \mathbb{N}$ and $p_{1}, \ldots, p_{k}$ are not necessarily distinct primes;

(c) $G \cong L \times F \times \prod_{i=1}^{k} \mathbb{Z}\left(p_{i}^{\infty}\right)$, where $k \in \mathbb{N}, F$ is a finite abelian group, $L$ is a subgroup of $\mathbb{Q}^{m}(m \in \mathbb{N})$ and $p_{1}, \ldots, p_{k}$ are not necessarily distinct primes;

(d) $G$ contains no infinite direct sum of non-trivial subgroups;

(e) $G$ contains no subgroup $H$, which is a direct sum of $|G|$-many non-trivial subgroups.

Proof. (a) $\rightarrow$ (b). Let $D(G)$ be the divisible hull of $G$. Then, $r(D(G))=r(G)$, since $r_{0}(D(G))=r_{0}(G)$ and $r_{p}(D(G))=r_{p}(G)$ by the fact that $G$ is essential in $D(G)$ (see [17] (Lemma 24.3)). Therefore, (a) implies that $r(D(G))<\infty$. Now, (b) follows from the structure theorem for divisible abelian groups.

(b) $\rightarrow$ (c) Assume wlogthat $G$ is a subgroup of $H=\mathbb{Q}^{m} \times D$, where $D=\prod_{i=1}^{k} \mathbb{Z}\left(p_{i}^{\infty}\right)$. Then, the torsion subgroup $t(G)$ of $G$ is a subgroup of $D$; hence, $t(G)=F \times T$ for subgroup $T \cong \prod_{s=1}^{t} \mathbb{Z}\left(p_{i_{s}}^{\infty}\right)$ of $G$ and for appropriate $1 \leq i_{1}<\ldots<i_{t} \leq k$ and a finite subgroup $F$ of $\prod_{i=1}^{k} \mathbb{Z}\left(p_{i}^{\infty}\right)$. Since $T$ is a divisible subgroup of $G$, it splits in $G$. Therefore, there exists a subgroup $G_{1}$ (containing $F$ ) of $G$, such that $G=G_{1} \times T$. Note that $t\left(G_{1}\right) \cong t(G / T)=t(G) / T \cong F$, since $T$ is a torsion group. By a theorem of Kulikov [17] (Theorem 27.5), the torsion part of an abelian group splits when it is finite, so we can write $G_{1}=t\left(G_{1}\right) \times G_{2}$, where $G_{2}$ is a torsion-free subgroup of $G_{1}$ (isomorphic to $G_{1} / t\left(G_{1}\right) \cong G / t(G)$ ). Since $r_{0}\left(G_{2}\right) \leq r_{0}(G) \leq r_{0}(H)=m<\infty$, one has $D\left(G_{2}\right)=\mathbb{Q}^{m_{2}}$ for some $m_{2} \leq m$, so $G_{2}$ is isomorphic to a subgroup of $\mathbb{Q}^{m}$.

$(c) \rightarrow(d)$ is obvious and (d) $\rightarrow$ (e) is trivial.

To prove the implication of (e) $\rightarrow$ (a), assume for a contradiction that $r(G)$ is infinite. Then, $G$ contains a direct sum of $r(G)$-many non-trivial subgroups (it will be isomorphic to $\left.\left(\bigoplus_{r_{0}(G)} \mathbb{Z}\right) \oplus\left(\bigoplus_{p} \bigoplus_{r_{p}(G)} \mathbb{Z}_{p}\right)\right)$. To end the proof, it suffices to note that $|G|=r(G)$ whenever the latter cardinal is infinite [17].

It is important to note that while the $d$-rank is obviously monotone with respect to taking quotients, the rank is not (i.e., a quotient of a group may have a bigger rank that the group itself, as we shall see in a while).

A group $G$ of finite rank may have infinite $\varrho(G)($ e.g., $r(\mathbb{Q})=1$, while $\varrho(\mathbb{Q})=\omega$, witnessed by $r(\mathbb{Q} / \mathbb{Z})=\omega)$. In order to understand better the properties of the $d$-rank of rational groups, we introduce some specific rational groups.

Let $\mathbb{Q}_{p}$ be the subgroup of $\mathbb{Q}$ formed by all rational numbers having only powers of a given fixed prime number $p$ in the denominator. For a set $\pi$ of prime numbers, denote by $\mathbb{Q}_{\pi}$ the subgroup $\sum_{p \in \pi} \mathbb{Q}_{p}$ of $\mathbb{Q}$, i.e., $\mathbb{Q}_{\pi}$ consists of those rational numbers 
for which the primes from $\mathbb{P} \backslash \pi$ are excluded from denominators. For completeness, let $\mathbb{Q} \varnothing=\mathbb{Z}$.

Example 2.3. For $\pi \subseteq \mathbb{P}$, one has $r\left(\mathbb{Q}_{\pi}\right)=1$ and $\varrho\left(\mathbb{Q}_{\pi}\right)=|\pi|$. In particular, $\varrho\left(\mathbb{Q}_{\pi}^{n}\right)<\infty$ (for $n \in \mathbb{N}$ ) if and only if $\pi$ is finite. Actually, a torsion-free group $G$ has finite $d$-rank if and only if $G$ is isomorphic to a subgroup of $\mathbb{Q}_{\pi}^{n}$ for some $n \in \mathbb{N}$ and some finite $\pi \subseteq \mathbb{P}$ ([18] (Lemma 10.8)).

One can describe the non-r-disconnected discrete groups as follows:

Fact 2.4. ([18] (Lemma 10.12)) A discrete abelian group $G$ is non- $r$-disconnected (i.e., has $\varrho(G)<\infty$ ) if and only if $G \cong L \times F \times \prod_{i=1}^{k} \mathbb{Z}\left(p_{i}^{\infty}\right)$, where $L$ is a torsion-free non- $r$-disconnected group (i.e., isomorphic to a subgroup of $\mathbb{Q}_{\pi}^{n}$ for some finite $\pi \subseteq \mathbb{P}$ and $n \in \mathbb{N}), F$ is a finite abelian group, $k \in \mathbb{N}$ and $p_{1}, \ldots, p_{k}$ are not necessarily distinct primes.

In order to describe also the non-r-disconnected LCA groups, we need a fundamental fact from the structure theory of LCA groups.

Fact 2.5. According to the structure theory, an LCA group $G$ is topologically isomorphic to $\mathbb{R}^{n} \times H$, where $n \in \mathbb{N}$ and the group $H$ has a compact open subgroup $K$ ([19]). Therefore, the quotient group $D=H / K$ is discrete.

Example 2.6. Let $G$ be a non-r-disconnected LCA group.

(a) $G$ has a compact subgroup $K$, such that $G / K \cong \mathbb{R}^{n} \times L \times \prod_{i=1}^{k} \mathbb{Z}\left(p_{i}^{\infty}\right)$ for some $n, k \in \mathbb{N}$, a torsion-free discrete non- $r$-disconnected group $L$, and not necessarily distinct primes $p_{i}$.

(b) The quotient $\mathbb{R}^{n} \times L \times \prod_{i=1}^{k} \mathbb{Z}\left(p_{i}^{\infty}\right)$ does not depend on the choice of the compact group $K$ up to isomorphism in the following sense. If $K_{1}$ is another compact subgroup of $G$, such that $G / K_{1} \cong \mathbb{R}^{n_{1}} \times L_{1} \times \prod_{j=1}^{k_{1}} \mathbb{Z}\left(q_{j}^{\infty}\right)$ with $n_{1}, k_{1} \in \mathbb{N}$, a torsion-free discrete non- $r$-disconnected group $L_{1}$, and not necessarily distinct primes $q_{j}$, then $G / K \cong G / K_{1}$ (so $n_{1}=n, L_{1} \cong L, k_{1}=k$ and $p_{i}=q_{j}$ for all $i=1,2, \ldots, k$ and an appropriate permutation of the primes $q_{j}$ ).

Proof. (a) Indeed, as we saw above, there exists a closed subgroup $H$ of $G$ with a compact open subgroup $K$, such that $G=\mathbb{R}^{n} \times H$, so $N=\mathbb{R}^{n} \times K$ is an open subgroup of $G$. By our hypothesis, $H / K \cong G / N$ has finite $d$-rank. By Fact 2.4, $H / K \cong L \times F \times \prod_{i=1}^{k} \mathbb{Z}\left(p_{i}^{\infty}\right)$, where $L$ is a a torsion-free discrete non- $r$-disconnected group, $F$ is a finite group and $p_{1}, \ldots, p_{k}$, with $k \in \mathbb{N}$, are not necessarily distinct primes. Choosing $K$ a bit larger, we can assume without loss of generality that $F=0$. Since $G / K \cong \mathbb{R}^{n} \times H / K$, we are done.

(b) Since $K+K_{1}$ is a compact subgroup of $G$ containing $K$ and $K_{1}$ as open subgroups, both indexes $\left[\left(K+K_{1}\right): K\right]$ and $\left[\left(K+K_{1}\right): K_{1}\right]$ are finite. Therefore, 
$F=\left(K+K_{1}\right) / K$ is a finite subgroup of $G / K$, and similarly, $F_{1}=\left(K+K_{1}\right) / K_{1}$ is a finite subgroup of $G / K_{1}$. Moreover,

$$
(G / K) / F \cong G /\left(K+K_{1}\right) \cong\left(G / K_{1}\right) / F_{1}
$$

Since $F$ is a finite subgroup of $t(G / K) \cong \prod_{i=1}^{k} \mathbb{Z}\left(p_{i}^{\infty}\right)$, it is easy to see that $t(G / K) / F \cong t(G / K)$. Similarly, $t\left(G / K_{1}\right) / F_{1} \cong t\left(G / K_{1}\right)$. This yields the isomorphisms $(G / K) / F \cong G / K$ and $\left(G / K_{1}\right) / F_{1} \cong G / K_{1}$. Now, the isomorphisms (6) yield the desired isomorphism $G / K \cong G / K_{1}$. These isomorphisms take the connected component $c(G / K) \cong \mathbb{R}^{n}$ of $G / K$ to the the connected component $c\left(G / K_{1}\right) \cong \mathbb{R}^{n_{1}}$ of $G / K_{1}$; in particular, $n_{1}=n$. Moreover, these isomorphisms takes the torsion subgroup $t(G / K) \cong \prod_{i=1}^{k} \mathbb{Z}\left(p_{i}^{\infty}\right)$ of $G / K$ to the torsion subgroup $t\left(G / K_{1}\right) \cong \prod_{j=1}^{k_{1}} \mathbb{Z}\left(q_{j}^{\infty}\right)$ of $G / K_{1}$. Hence, $k_{1}=k, p_{i}=q_{j}$ for all $i=1,2, \ldots, k$ and an appropriate permutation of the primes $q_{j}$. Finally, the isomorphism induces an isomorphism of the quotients $(G / K) /(c(G / K) \times t(G / K)) \cong L$ and $\left(G / K_{1}\right) /\left(c\left(G / K_{1}\right) \times t\left(G / K_{1}\right)\right) \cong L_{1}$. Therefore, we deduce $L_{1} \cong L$.

We need a property of the discrete rank $\varrho(G)$, when the group $G$ is LCA and $r$-disconnected. Since it is related to the rank of discrete quotients of $G$, it has also a connection to the compact covering number $k(G)$, as Theorem 2.7 shows.

Theorem 2.7. Let $G$ be an r-disconnected group. Then, $k(G) \geq \varrho(G)$. If $G$ is $L C A$, then:

(a) G has an open $\sigma$-compact subgroup $L$ with $r(G / L)=\varrho(G)$.

(b) every discrete quotient of $G$ has a size at most $\varrho(G)$.

(c) there exists a compact subgroup $N$ of $G$, such that $G / N \cong \mathbb{R}^{n} \times D$ for some discrete abelian group $D$ with $|D|=\varrho(G)$ and $n \in \mathbb{N}$.

(d) $k(G)=\varrho(G)$.

Proof. Assume that $G=\bigcup_{i \in I} K_{i}$, where each $K_{i}$ is compact. Then, for every open subgroup $H$ of $G$ with infinite $G / H$, the quotient map $q: G \rightarrow G / H$ takes each $K_{i}$ to a finite subset of $G / H$. Since $G / H$ is infinite, one has $|G / H| \leq|I|$. This proves that $\varrho(G) \leq k(G)$.

Now, suppose that $G$ is LCA. We may assume without loss of generality that $G=\mathbb{R}^{n} \times H$ with $n, H$ and $K$, as in Fact 2.5. Then, the subgroup $L=\mathbb{R}^{n} \times K$ of $G$ is open and $\sigma$-compact. Moreover, $G / L \cong H / K$ is discrete. Every open subgroup of $G$ contains $\mathbb{R}^{n} \times\{0\}$, so having the form $\mathbb{R}^{n} \times O$, for some open subgroup $O$ of $H$. Hence, our hypothesis of $r$-disconnectedness on $G$ implies that $H$ is $r$-disconnected, as well, and $\varrho(G)=\varrho(H) \geq r(H / K)=|H / K|$.

(a) To see that $\varrho(G)=\varrho(H) \leq r(H / K)$, take any open subgroup $N$ of $H$. We can assume wlog that $N$ is contained in $K$. Then, the quotient $K / N$ is both discrete and compact, hence finite. Therefore, $r(H / N)=r(H / K)$ as the quotient $K / N$ is finite, while $r(H / N)$ and $r(H / K)$ are both infinite. 
(b) Let $O \leq G$ be an open subgroup. If $r(G / O)<\infty$, then $|G / O| \leq \omega \leq \rho(G)$; otherwise, if $r(G / O)=\infty$, then $|G / O|=r(G / O) \leq \rho(G)$.

(c) follows from the above argument.

(d) We only have to check that $k(G) \leq \varrho(G)$. Since $\mathbb{R}^{n}$ is $\sigma$-compact and $K$ is compact, one has $k(G) \leq \omega \cdot|D|=\omega \cdot \varrho(G)=\varrho(G)$.

\section{Some General Properties of Compatible Topologies}

Let $(G, \tau)$ be a topological group. Another group topology $v$ on $G$ is said to be compatible for $(G, \tau)$ if it has the same character group as the original one, that is if $(G, v)^{\wedge}=(G, \tau)^{\wedge}$.

We start with the following general facts providing easy upper bounds for $|\mathscr{C}(G, \tau)|$ :

Proposition 3.1. If $(G, \tau)$ is Mackey, then:

(a) $|\mathscr{C}(G, \tau)| \leq 2^{|\tau|}$; so $|\mathscr{C}(G, \tau)| \leq 2^{2^{\mathrm{w}(G)}}$, where $\mathrm{w}(G)$ denotes the weight of $G$;

(b) if $\mathscr{C}_{\kappa}(G)$ denotes the subset of the compatible topologies of local weight $\leq \kappa$, then $\left|\mathscr{C}_{\mathcal{K}}(G)\right| \leq|\tau|^{\kappa}$. In particular, the cardinality of the set of metrizable compatible topologies is not greater than $|\tau|^{\omega}$.

Proof. (a) For the first inequality, it suffices to note that any compatible group topology is a subset of $\tau$. For the second assertion, note that $|\tau| \leq 2^{\mathrm{w}(G)}$.

(b) follows from the fact that a group topology with local weight $\leq \kappa$ is completely determined by the assignment of a family of size $\leq \kappa$ that forms its local base at zero.

In order to understand the structure (in particular, the size) of the poset $\mathscr{C}(G)$ for a topological group $G$, we relate $\mathscr{C}(G)$ to the corresponding posets $\mathscr{C}(H)$ and $\mathscr{C}(G / H)$ for convenient subgroups $H<G$.

Proposition 3.2. Let $G=H_{1} \times H_{2}$ be a locally quasi-convex group. Then,

$$
\mathscr{C}\left(H_{1}\right) \times \mathscr{C}\left(H_{2}\right) \longrightarrow \mathscr{C}(G),\left(v_{1}, v_{2}\right) \longmapsto v_{1} \times v_{2}
$$

is a poset embedding.

Proof. This mapping is injective and preserves the order. It remains to show that it is well defined. Therefore, let $\tau$ be the original topology on $G$ and $\tau_{1}, \tau_{2}$ the induced topologies on $H_{1}$ and $H_{2}$ respectively. Take $v_{1} \in \mathscr{C}\left(H_{1}, \tau_{1}\right)$ and $v_{2} \in \mathscr{C}\left(H_{2}, \tau_{2}\right)$. Then, $v_{1} \times v_{2}$ is again a locally quasi-convex topology on $G=H_{1} \times H_{2}$. Clearly,

$$
\left(H_{1} \times H_{2}, v_{1} \times v_{2}\right)^{\wedge}=\left(H_{1}, v_{1}\right)^{\wedge} \times\left(H_{2}, v_{2}\right)^{\wedge}=\left(H_{1}, \tau_{1}\right)^{\wedge} \times\left(H_{2}, \tau_{2}\right)^{\wedge}=(G, \tau)^{\wedge}
$$

so $v_{1} \times v_{2}$ is compatible. The assertion follows. 
A subgroup $H$ of a topological abelian group $G$ is called:

- dually closed if for every $x \in G \backslash H$, there exists $\chi \in G^{\wedge}$, such that $\chi(H)=\{0\}$ and $\chi(x) \neq 0$;

- dually embedded if each continuous character of $H$ can be extended to a continuous character of $G$.

Remark 3.3. It is well known that if $H$ is an open subgroup, it is dually closed and dually embedded, but in general, a closed subgroup need not have these properties.

(a) It is easy to see that a subgroup $H$ of a topological group $(G, \tau)$ is dually closed if and only if $H$ is $\tau^{+}$-closed.

(b) It is straightforward to prove that if $G=H_{1} \oplus H_{2}$ is equipped with the product topology, then $H_{1}$ and $H_{2}$ are dually embedded in $G$. Moreover, $H_{1}$ and $H_{2}$ are dually closed in $G$ precisely when $H_{1}$ and $H_{2}$ are maximally almost periodic.

(c) It follows from Item (a) that a pair of compatible topologies shares the same dually-closed subgroups.

Lemma 3.4. Let $(G, \tau)$ be a topological abelian group and $H$ a dually-closed and dually-embedded subgroup. If $M \subseteq H$ is quasi-convex in $H$, it is also quasi-convex in $G$.

Proof. We must check that every $x \in G \backslash M$ can be separated from $M$ by means of a continuous character. Consider two cases:

(a) $x \in H \backslash M$. Then, there exists $\chi \in H^{\wedge}$, such that $\chi(M) \subseteq \mathbb{T}_{+}$and $\chi(x) \notin \mathbb{T}_{+}$. Now, any extension of $\chi$, say $\tilde{\chi} \in G^{\wedge}$, does the job.

(b) $x \in G \backslash H$. Since $H$ is dually closed, there exists $\xi \in G^{\wedge}$, such that $\xi(H)=\{0\}$ and $\xi(x) \neq 0$. A suitable multiple of $\xi$ gives a character that separates $x$ from $M$.

Proposition 3.5. [20] (1.4) Every compact subgroup of a maximally almost periodic group is dually embedded and dually closed.

Proposition 3.6. Let $(G, \tau)$ be a locally quasi-convex group and $H$ an open subgroup. Then, there exists a canonical poset embedding:

$$
\Psi: \mathscr{C}\left(H,\left.\tau\right|_{H}\right) \longrightarrow \mathscr{C}(G, \tau)
$$

Proof. Fix $(H, v) \in \mathscr{C}\left(H,\left.\tau\right|_{H}\right)$, and let $\mathcal{N}_{H, v}(0)$ be a basis of quasi-convex zero neighborhoods for $v$. If $\mathcal{N}_{H, v}(0)$ is considered as a basis of zero neighborhoods in $G$, we obtain a new group topology $\Psi(v)$ on $G$, for which $H$ is an open subgroup. According to $3.4, \Psi(v)$ is locally quasi-convex.

Since $H$ is an open subgroup of $G$ both w.r.t. to $\tau$ and to $\Psi(v)$ and since a homomorphism $\chi: G \rightarrow \mathbb{T}$ is continuous iff its restriction to an open subgroup is continuous, it is sufficient to prove that $\left(H,\left.\Psi(v)\right|_{H}\right)$ is compatible with $\left(H,\left.\tau\right|_{H}\right)$. Obviously, $\left.\Psi(v)\right|_{H}=v \in \mathscr{C}\left(H,\left.\tau\right|_{H}\right)$; hence, the assertion follows. 
Remark 3.7. In the sequel, we denote by $Q(\tau)$ the quotient topology of a topology $\tau$ (the quotient group will always be quite clear from the context).

Let $H$ be a dually-closed subgroup of the Hausdorff abelian group $G$. By Remark 3.3(c), $H$ is $\tau$ closed for every topology $\tau \in \mathscr{C}(G)$. Hence, for $\tau \in \mathscr{C}(G)$, the group topology $Q(\tau)$ is Hausdorff and:

$$
Q: \mathscr{C}(G) \longrightarrow \mathcal{T}(G / H), \tau \longmapsto Q(\tau)
$$

is an order-preserving mapping.

Of course, $Q$ is in general not injective. The situation improves when $K$ is a compact subgroup and $G$ is a locally quasi-convex Hausdorff group (see Theorem 3.15).

The next fact is probably known, but hard to find in the literature; hence, we prefer to give a proof for the reader's convenience.

Lemma 3.8. Let $(G, \tau)$ be a MAP abelian group and $H$ a dually-closed subgroup. Then, for the quotient $G / H$, one has $Q(\tau)^{+}=Q\left(\tau^{+}\right)$, i.e., the Bohr topology of the quotient coincides with the quotient topology of the Bohr topology $\tau^{+}$.

Proof. Since both $Q(\tau)^{+}$and $Q\left(\tau^{+}\right)$are precompact group topologies on $G / H$, it is sufficient to check that they are compatible. To this end, note that $Q(\tau)^{+} \geq Q\left(\tau^{+}\right)$, since $Q(\tau) \geq Q\left(\tau^{+}\right)$and $Q\left(\tau^{+}\right)$is precompact. To see that they are compatible, take a $Q(\tau)^{+}$-continuous character $\chi: G / H \rightarrow \mathbb{T}$. Then, it is also $Q(\tau)$-continuous. Hence, the composition with the canonical projection $q: G \rightarrow G / H$ produces a $\tau$-continuous character $\xi=\chi \circ q$ of $G$. Since $\xi$ is $\tau^{+}$-continuous, as well, from the factorization $\xi=\chi \circ q$, we deduce that $\chi$ is also $Q\left(\tau^{+}\right)$-continuous. Hence, $Q(\tau)^{+} \leq Q\left(\tau^{+}\right)$.

Next, we are interested in embedding $\mathscr{C}(G / H)$ into $\mathscr{C}(G)$. The following notation will be used in the sequel:

Notation 3.9. Let $H$ be a closed subgroup of the topological abelian group $(G, \tau)$. Denote by $q: G \rightarrow G / H$ the canonical projection. Further, for a group topology $\theta \in \mathcal{T}(G / H)$, we denote by $q^{-1}(\theta)$ the initial topology, namely the group topology $\left\{q^{-1}(O): O \in \theta\right\}$. It is straightforward to prove that whenever $\theta$ is locally quasi-convex, then $q^{-1}(\theta)$ is locally quasi-convex, as well.

Theorem 3.10. Let $H$ be a dually-closed subgroup of the locally quasi-convex Hausdorff group $(G, \tau)$. The mapping:

$$
\Theta: \mathscr{C}(G / H, Q(\tau)) \longrightarrow \mathscr{C}(G, \tau), \theta \longrightarrow q^{-1}(\theta) \vee \tau^{+}
$$

is a poset embedding with left inverse $Q$. 
Proof. According to 3.9, $\Theta(\theta)$ is a locally quasi-convex group topology on $G$ and finer than $\tau^{+}$, hence Hausdorff.

Before proving that $\Theta(\theta)$ is compatible, we show that $Q \circ \Theta(\theta)=\theta$. This will imply (once it is shown that $\Theta$ is well defined) that $Q$ is a left inverse for $\Theta$, and hence, $\Theta$ is injective. A neighborhood basis of zero in $(G, \Theta(\theta))$ is given by sets of the form $V=q^{-1}(U) \cap W$ where $W$ is a neighborhood of zero in $\left(G, \tau^{+}\right)$and $U$ a neighborhood of zero in $(G / H, \theta)$. Observe that $q(V)=U \cap q(W)$. This implies the first equality in the following chain of equalities:

$$
Q(\Theta(\theta)))=\theta \vee Q\left(\tau^{+}\right) \stackrel{3.8}{=} \theta \vee Q(\tau)^{+}=\theta
$$

(the last equality follows from $\theta \geq Q(\tau)^{+}$).

Let us show now that $\Theta(\theta)$ is compatible. Since $\Theta(\theta) \geq \tau^{+}$, we only need to show that if $\chi \in(G, \Theta(\theta))^{\wedge}$, then $\chi$ is also continuous with respect to $\tau$. It is easy to check that $\left.\Theta(\theta)\right|_{H}=\left.\tau^{+}\right|_{H}$. Hence, we obtain that $\left.\chi\right|_{H}:\left(H,\left.\tau^{+}\right|_{H}\right) \rightarrow \mathbb{T}$ is continuous. Thus, $\left.\chi\right|_{H}$ is a continuous character of the precompact group $\left(H,\left.\tau^{+}\right|_{H}\right)$, which is dually embedded in $\left(G, \tau^{+}\right)$. Hence, there exists a continuous character $\chi_{1} \in\left(G, \tau^{+}\right)^{\wedge}$, which extends $\chi$. Since $\chi_{1} \in\left(G, \tau^{+}\right)^{\wedge}=(G, \tau)^{\wedge}$, it is sufficient to show that $\chi-\chi_{1} \in(G, \tau)^{\wedge}$ or, equivalently, we may suppose that $\chi \in H^{\perp}$.

Hence, $\bar{\chi}:(G / H, \underbrace{Q(\Theta(\theta))}_{=\theta}) \rightarrow \mathbb{T}, x+H \mapsto \chi(x)$ is well defined and continuous.

Since $\theta$ is compatible with $Q(\tau)$, we deduce that $\bar{\chi}:(G / H, Q(\tau)) \rightarrow \mathbb{T}$ is continuous, and hence, $\chi=\bar{\chi} \circ q:(G, \tau) \rightarrow \mathbb{T}$ is continuous.

This completes the proof.

Since open or compact subgroups are dually closed, the above theorem gives:

Corollary 3.11. Let $G$ be a locally quasi-convex group, and let $H$ be an open or a compact subgroup of $G$. Then, $\Theta: \mathscr{C}(G / H) \rightarrow \mathscr{C}(G)$ is a poset embedding.

Remark 3.12. Let $(G, \tau)$ be a locally quasi-convex group, and let $H$ be an open subgroup of $G$. The images $\Theta(\mathscr{C}(G / H))$ and $\Psi(\mathscr{C}(H))$ in $\mathscr{C}(G)$ of both embeddings, obtained in Theorem 3.6 and Corollary 3.11, meet in a singleton, namely:

$$
\Theta(\mathscr{C}(G / H)) \cap \Psi(\mathscr{C}(H))=\left\{\mathcal{T}_{0} \vee \tau^{+}\right\}
$$

where $\mathcal{T}_{0}$ is the topology on $G$ with neighborhood basis $\{H\}$.

Indeed, one can see first that if $\mathcal{T} \in \Psi(\mathscr{C}(H))$, then $\mathcal{T} \geq \mathcal{T}_{0} \vee \tau^{+}$. On the other hand, if $\mathcal{T} \in \Theta(\mathscr{C}(G / H))$, then $\mathcal{T}=\Theta(\theta) \leq \Theta\left(\delta_{G / H}\right)=\mathcal{T}_{0} \vee \tau^{+}$ for some $\theta \in \mathscr{C}(G / H)$, where $\delta_{G / H}$ denotes the discrete topology on $G / H$. Combining both inclusions, we obtain $\mathcal{T}=\mathcal{T}_{0} \vee \tau^{+}$. On the other hand, to see that the topology $\mathcal{T}_{0} \vee \tau^{+}$is an element of the intersection, it suffices to realize that $\mathcal{T}_{0} \vee \tau^{+}=\Psi\left(\left.\tau_{+}\right|_{H}\right)=\Theta\left(\delta_{G / H}\right)$. 
In the proof of the next result, namely that for a locally quasi-convex group $G$ and a compact subgroup $K$ of $G$, the posets $\mathscr{C}(G)$ and $\mathscr{C}(G / K)$ are isomorphic, we need the following results from [21]:

Theorem 3.13. Let $(G, \tau)$ be a locally quasi-convex Hausdorff group, and let $K$ be a subgroup of $G$.

(a) [21] (Theorem (3.5)) If $\left(K,\left.\tau^{+}\right|_{K}\right)$ is compact, then $\left.\tau\right|_{K}=\left.\tau^{+}\right|_{K}$, in particular $\left(K,\left.\tau\right|_{K}\right)$ is compact.

(b) [21] (Theorem (2.7)) If $K$ is compact, then $G / K$ is a locally quasi-convex Hausdorff group.

Further, we need:

Lemma 3.14 (Merzon). Let $\tau_{1} \leq \tau_{2}$ be group topologies on a group $G$, and let $H$ be a subgroup of $G$. If the subspace topologies $\left.\tau_{1}\right|_{H}=\left.\tau_{2}\right|_{H}$ and the quotient topologies $Q\left(\tau_{1}\right)=Q\left(\tau_{2}\right)$ coincide, then $\tau_{1}=\tau_{2}$.

Theorem 3.15. Let $(G, \tau)$ be a locally quasi-convex Hausdorff group and $K$ a compact subgroup of $G$. Then:

$$
\Theta: \mathscr{C}(G / K) \longrightarrow \mathscr{C}(G) \text { and } Q: \mathscr{C}(G) \longrightarrow \mathscr{C}(G / K)
$$

are mutually inverse poset isomorphisms.

Proof. Let us show first that $Q: \mathscr{C}(G) \rightarrow \mathscr{C}(G / K), \theta \mapsto Q(\theta)$ is well defined. According to 3.13(b), $Q(\theta)$ is a locally quasi-convex Hausdorff group topology. In order to show that $Q(\theta)$ is compatible for $\theta \in \mathscr{C}(G)$, we fix a continuous character $\chi:(G / K, Q(\theta)) \rightarrow \mathbb{T}$. Let $q: G \rightarrow G / K$ be the quotient homomorphism. Then, $\chi \circ q:(G, \theta) \rightarrow \mathbb{T}$ is continuous. Since $\theta$ is compatible for $(G, \tau)$, also $\chi \circ q:(G, \tau) \rightarrow \mathbb{T}$ is continuous, and hence, $\chi:(G, Q(\tau)) \rightarrow \mathbb{T}$ is continuous. On the other hand, $\theta \geq \tau^{+}$, and hence, $Q(\theta) \geq Q\left(\tau^{+}\right)=Q(\tau)^{+}$. Combining both conclusions, we obtain that $Q(\theta)$ is compatible for $(G, Q(\tau))$.

Taking into account 3.10, it is sufficient to prove that $\Theta \circ Q=\operatorname{id}_{\mathscr{C}(G)}$.

Therefore, fix $\theta \in \mathscr{C}(G)$. The topology $\Theta(Q(\theta))=q^{-1}(Q(\theta)) \vee \tau^{+}$is coarser than $\theta$, so applying Merzon's Lemma, it is sufficient to show that $\theta$ and $q^{-1}(Q(\theta)) \vee \tau^{+}$coincide on $K$ and on $G / K$. Since $\theta^{+}=\tau^{+}$and since $\left(H,\left.\tau^{+}\right|_{K}\right)$ is compact, we obtain by Theorem $3.13\left(\right.$ a) that $\left(K,\left.\theta\right|_{K}\right)=\left(K,\left.\theta^{+}\right|_{K}\right)$ is compact. Hence: $\left.\theta\right|_{K} \geq\left.\Theta(Q(\theta))\right|_{K} \geq\left.\tau^{+}\right|_{K}=\left.\theta^{+}\right|_{K}=\left.\theta\right|_{K}$ imply that $\left.\Theta(Q(\theta))\right|_{K}=\left.\theta\right|_{K}$. For the quotient topologies, the following holds by Theorem 3.10: $Q(\Theta(Q(\theta)))=$ $(Q \circ \Theta)(Q(\theta))=Q(\theta)$. Combining the partial results, the theorem is proven.

Since every locally compact abelian group is locally quasi-convex, from Theorem 3.15, we immediately obtain: 
Corollary 3.16. Let $G$ be a locally compact abelian group and $K$ a compact subgroup of $G$. Then, $\mathscr{C}(G) \cong \mathscr{C}(G / K)$.

Corollary 3.17. Let $G$ be a $\sigma$-compact group with an open compact subgroup. Then, there is a poset embedding $\mathscr{C}(G) \hookrightarrow \mathfrak{F}_{\omega} ;$ in particular, $|\mathscr{C}(G)| \leq 2^{\text {c }}$.

Proof. Let $K$ be an open compact subgroup of $G$. Without loss of generality, we may assume that $G$ is not compact, and hence, $G / K$ is infinite.

As a consequence of the above theorem, $Q: \mathscr{C}(G) \rightarrow \mathscr{C}(G / K)$ is a poset isomorphism. Since $G / K$ is countable and discrete, 1.6 establishes a poset embedding $\mathscr{C}(G / K) \hookrightarrow \mathcal{T}(G / K) \hookrightarrow \mathfrak{F}_{\omega}$.

Note that a group $G$ as in the above corollary is necessarily locally compact. We shall see in the sequel (see Example 2.6) that unless $G$ has a very special structure, one has actually a quasi-isomorphism between $\mathscr{C}(G)$ and $\mathfrak{F}_{\omega}$, in particular $|\mathscr{C}(G)|=2^{\mathfrak{c}}$.

\section{Compatible Topologies for Discrete Abelian Groups}

We intend to reduce the study of the poset $\mathscr{C}(G)$ for a LCA group $G$ to the case of infinite direct sums of countable groups. This is why, throughout the first part of this section, $\gamma$ will denote an infinite cardinal and $C_{\alpha}$ will be a non-zero countable group for every $\alpha<\gamma$. Our intention is to show that for the discrete group $G=\bigoplus_{\alpha<\gamma} C_{\alpha}$, the poset $\mathscr{C}(G)$ is quasi-isomorphic to $\mathfrak{F}_{\gamma}$ (so, it contains an anti-chain of the maximal possible size $2^{2^{\gamma}}$ (note that $\gamma=|G|$ ), in particular $\mathscr{C}(G)$ has width and size $2^{2^{\gamma}}$ ).

In order to get (many) group topologies on $G$, we need a frequently-used standard construction based on filters on $\gamma$.

Notation 4.1. Every free filter $\varphi$ on $\gamma$ defines a topology $\tau_{\varphi}$ on $G$ with a base $\left\{W_{B}: B \in \varphi\right\}$ of neighborhoods of zero, where:

$$
W_{B}=\bigoplus_{\alpha \in B} C_{\alpha}
$$

(here, we are identifying the direct sum defining $W_{B}$ with a subgroup of $G$ in the obvious way, by adding zeros in the coordinates $\alpha \notin B$ ). Using the fact that each $W_{B}$ is a subgroup of $G$ and $W_{B_{1}} \cap W_{B_{2}}=W_{B_{1} \cap B_{2}}$ for $B_{1}, B_{2} \in \varphi$, one can easily prove that $\tau_{\varphi}$ is a Hausdorff group topology on $G$. Moreover, $\tau_{\varphi}$ is locally quasi-convex, since every basic open neighborhood $W_{B}$ is an open subgroup, hence quasi-convex.

Finally, let us mention the fact (although it will not be used here) that, according to [22], $\tau_{\varphi}$ is complete when $\varphi$ is an ultrafilter.

Lemma 4.2. Let $\delta$ denote the discrete topology on $G$. The mapping:

$$
\Xi: \mathfrak{F}_{\gamma} \longrightarrow \mathscr{C}(G), \varphi \longmapsto \tau_{\varphi} \vee \delta^{+}
$$


is a poset embedding.

Proof. Obviously $\delta^{+} \leq \tau_{\varphi} \vee \delta^{+} \leq \delta$, so the topology $\tau_{\varphi} \vee \delta^{+}$is compatible. Since $\delta^{+}$is precompact, $\delta^{+}$is also locally quasi-convex. Moreover, $\tau_{\varphi} \vee \delta^{+}$is locally quasi-convex, as proven in Notation 4.1. This shows that $\Xi$ is well defined.

It is obvious that $\Xi$ preserves the order. Let us show that $\Xi$ is injective: To that end we fix $\varphi, \psi \in \mathfrak{F}_{\gamma}$ and assume that $\tau_{\varphi} \vee \delta^{+}=\tau_{\psi} \vee \delta^{+}$, in particular $\tau_{\varphi} \vee \delta^{+} \leq \tau_{\psi} \vee \delta^{+}$, and hence, $\tau_{\varphi} \leq \tau_{\psi} \vee \delta^{+}$. Let $B \in \varphi$, then $W_{B} \in \tau_{\varphi}$, so $W_{B} \in \tau_{\psi} \vee \delta^{+}$by our hypothesis. Then, there exist $B^{\prime} \in \psi$ and a finite set $F \subseteq G^{\wedge}$, such that $U:=W_{B^{\prime}} \cap F^{\triangleleft} \subseteq W_{B}$. Since $U \subseteq W_{B^{\prime}}$, as well, we conclude that $U \subseteq W_{B} \cap W_{B^{\prime}}=W_{B \cap B^{\prime}}$. Since $U$ is a neighborhood of zero in the Bohr topology of the subgroup $W_{B^{\prime}}$, this proves that also $W_{B \cap B^{\prime}}$ is a neighborhood of zero in the Bohr topology of $W_{B^{\prime}}$. This means that $W_{B \cap B^{\prime}}$ is an open subgroup of $W_{B^{\prime}}$ equipped with the Bohr topology. Since every open subgroup in a precompact topology must have finite index, we deduce that $W_{B \cap B^{\prime}}$ has finite index in $W_{B^{\prime}}$. Therefore, $W_{B^{\prime}} / W_{B \cap B^{\prime}} \cong \bigoplus_{\alpha \in B^{\prime} \backslash B} C_{\alpha}$ is finite. Consequently, also the set $S=B^{\prime} \backslash B$ is finite. Since $\gamma \backslash S \in \psi$, we conclude that also $B^{\prime \prime}:=B^{\prime} \backslash S=B^{\prime} \cap(\gamma \backslash S) \in \psi$. On the other hand, $B^{\prime \prime} \backslash B=\varnothing$, i.e., $B^{\prime \prime} \subseteq B$. This proves that $B \in \psi$. Therefore, $\varphi \subseteq \psi$. The other inclusion is proven analogously.

Remark 4.3. Observe that $\Xi(\mathcal{P}(\gamma))=\delta$.

Corollary 4.4. For $G$ as above, the sets $\mathscr{C}(G)$ and $\mathfrak{F}_{\gamma}$ are quasi-isomorphic, in particular width $(\mathscr{C}(G))=|\mathscr{C}(G)|=2^{2^{|G|}}$.

Further, $\mathscr{C}(G)$ has chains of size (at least) $\gamma$.

Proof. According to 4.2 and 1.6, the sets $\mathscr{C}(G)$ and $\mathfrak{F}_{\gamma}$ are quasi-isomorphic. Since $|G|=\gamma$ and since there are $2^{2^{\gamma}}$ different free ultrafilters in $\mathfrak{F}_{\gamma}$, the first assertion follows.

$\mathfrak{F}_{\gamma}$ has chains of length $\gamma$. Indeed, one can easily produce a chain of length $\gamma$ in $\mathfrak{F}_{\gamma}$ by using any partition of $\gamma$ in $\gamma$ pairwise disjoint sets of size $\gamma$ each.

Theorem 4.5. Let $G$ be a discrete abelian group of infinite rank. Then, $\mathscr{C}(G) \stackrel{\text { q.i. }}{\cong} \mathfrak{F}_{|G|}$ holds. In particular, $G$ admits $2^{2^{|G|}}$ pairwise incomparable compatible group topologies and $|\mathscr{C}(G)|=2^{2^{|G|}}$.

Proof. Let $\gamma=|G|$. According to 1.6, there are poset embeddings:

$$
\mathscr{C}(G) \longrightarrow \mathcal{T}(G) \longrightarrow \mathfrak{F}_{\gamma}
$$

It is easy to see that $G$ has a subgroup $H$ isomorphic to a direct sum $\bigoplus_{\alpha<\gamma} C_{\alpha}$, where each $C_{\alpha}$ is a non-trivial countable group (actually, it can be taken to be a cyclic 
group). According to Lemma 4.2 and Proposition 3.6, there are poset embeddings $\Xi: \mathfrak{F}_{\gamma} \rightarrow \mathscr{C}(H)$ and $\Psi: \mathscr{C}(H) \rightarrow \mathscr{C}(G)$. This proves that $\mathscr{C}(G) \stackrel{\text { q.i. }}{\cong} \mathfrak{F}_{|G|}$. The second assertion follows from (1).

Corollary 4.6. Suppose that $H$ is a discrete abelian group for which $\mathfrak{F}_{\omega}$ does not embed in $\mathscr{C}(H)$. Then, $H$ has finite d-rank; in particular, $H$ is countable.

Proof. If $H$ has infinite $d$-rank, then 4.5 implies the existence of a poset embedding $\mathfrak{F}_{|H|} \rightarrow \mathscr{C}(H)$. Hence, the hypothesis implies that $H$ has finite rank.

The case of finite rank abelian groups will be considered separately in [16].

\section{Proofs of Theorem A, Theorem B and Corollary C}

We intend to apply the results obtained so far to describe the poset of all compatible group topologies for $r$-disconnected groups (in particular, for non- $\sigma$-compact LCA groups).

Here comes the proof of Theorem A. It shows that for an LCA group $G$ with large discrete $\operatorname{rank} \varrho(G)$, the poset $\mathscr{C}(G)$ is quite large (in particular, $|\mathscr{C}(G)|<2^{2^{\varrho(G)}}$ implies, among other things, that $G$ is $\sigma$-compact).

Proof of Theorem A. Let $H$ be an open subgroup of the locally quasi-convex group $(G, \mathcal{T})$, such that $G / H$ has infinite rank. According to Corollary 3.11 and Theorem 4.5, there is a poset embedding $\mathfrak{F}_{|G / H|} \hookrightarrow \mathscr{C}(G)$. The assertion follows.

This theorem gives as a by-product a description of the LCA groups $G$, such that $\mathfrak{F}_{\omega}$ does not embed in $\mathscr{C}(G)$.

Corollary 5.1. If $G$ is an $L C A$ group, such that $\mathfrak{F}_{\omega}$ does not embed in $\mathscr{C}(G)$, then $G$ is non-r-disconnected (so $G$ contains a compact subgroup $K$, such that $G / K \cong \mathbb{R}^{n} \times$ $L \times \prod_{i=1}^{k} \mathbb{Z}\left(p_{i}^{\infty}\right)$ for some $n, m, k \in \mathbb{N}$, a subgroup $L$ of $\mathbb{Q}^{m}$ and not necessarily distinct primes $\left.p_{i}\right)$.

Proof. This follows from Example 2.6 and Theorem A.

Proof of Theorem B. First, we have to prove that if $G$ is an $r$-disconnected LCA group, then there exist poset embeddings as in formula (4). Since the existence of the first one was already established in Theorem $\mathrm{A}$, it remains to produce the embedding $\mathscr{C}(G) \hookrightarrow \mathfrak{F}_{\mathfrak{c} \cdot \varrho(G)}$. To this end, we use the isomorphism $\mathscr{C}(G) \cong \mathscr{C}\left(\mathbb{R}^{n} \times D\right)$ provided by the above fact. Since $\left|\mathbb{R}^{n} \times D\right|=\mathfrak{c} \cdot \varrho(G)$, Proposition 1.6 applies.

Now, assume that $G$ is totally disconnected. We have to prove that $\mathscr{C}(G) \stackrel{q . i .}{\cong} \mathfrak{F}_{Q(G)}$. According to Fact 2.5 , the LCA group $G$ is topologically isomorphic 
to $\mathbb{R}^{n} \times H$, where $n \in \mathbb{N}$ and the group $H$ has a compact open subgroup $K$. Since the quotient group $D=H / K$ is discrete and $G / K \cong \mathbb{R}^{n} \times D$, Corollary 3.16 gives $\mathscr{C}(G) \cong \mathscr{C}\left(\mathbb{R}^{n} \times D\right)$. According to our hypothesis, $G$ is totally disconnected, so $G$ cannot contain subgroups of the form $\mathbb{R}^{n}$ with $n>0$. Therefore, $G / K=D$ is discrete. Now, Corollary 3.16 implies that $\mathscr{C}(G) \cong \mathscr{C}(G / K)$. Moreover, $r(G / K)$ is infinite,

since $G$ is $r$-disconnected. Thus, $\mathscr{C}(G / K) \stackrel{q . i .}{\cong} \mathfrak{F}_{|G / K|}=\mathfrak{F}_{\varrho(G)}$ by Theorem 4.5.

Proof of Corollary C. We have to prove that if $G$ is non- $\sigma$-compact, then $\varrho(G)>\omega$, and there exists a poset embedding $\mathfrak{F}_{\varrho(G)} \hookrightarrow \mathscr{C}(G)$. By Theorem 2.7(d), we have $\varrho(G)=k(G)>\omega ;$ so, $G$ is $r$-disconnected, and Theorem A applies.

\section{Metrizable Separable Mackey Groups with Many Compatible Topologies}

For an abelian topological group $X$, we denote by $c_{0}(X)$ the subgroup of $X^{\mathbb{N}}$ consisting of all $\left(x_{n}\right) \in X^{\mathbb{N}}$, such that $x_{n} \rightarrow 0$ in $X$. We denote by $\mathfrak{p}$ and $\mathfrak{u}$ the product topology and the uniform topology of $X^{\mathbb{N}}$, respectively. We use $\mathfrak{p}_{0}$ and $\mathfrak{u}_{0}$ to denote the topologies induced by $\mathfrak{p}$ and $\mathfrak{u}$, respectively, on $c_{0}(X)$.

Throughout this section, let $X$ be a non-trivial compact connected metrizable group:

Theorem 6.1. [23] The group $G=\left(c_{0}(X), \mathfrak{u}_{0}\right)$ is a non-compact Polish connected Mackey group with $\mathfrak{u}_{0}^{+}=\mathfrak{p}_{0}$.

Observe that both $\mathfrak{u}_{0}$ and $\mathfrak{p}_{0}$ are metrizable and non-compact. In order to find more compatible topologies on $G$, we use the following construction.

Notation 6.2. For a subset $B$ of $\mathbb{N}$ and a neighborhood $U$ of 0 in $X$, let:

$$
P(B, U)=c_{0}(X) \cap\left(U^{B} \times X^{\mathbb{N} \backslash B}\right)
$$

The following properties of these sets will be frequently used in the sequel:

(a) $\bigcap_{i \in I} P\left(B_{i}, U\right)=P\left(\bigcup_{i \in I} B_{i}, U\right)$ for every subset $\left\{B_{i}: i \in I\right\} \subseteq \mathscr{P}(\mathbb{N})$;

(b) if $U \neq X$ and $P\left(B_{1}, U_{1}\right) \subseteq P(B, U)$, then $B \subseteq B_{1}$.

This follow directly from $X^{(\mathbb{N})} \cap\left(U_{1}^{B_{1}} \times X^{\left(\mathbb{N} \backslash B_{1}\right)}\right) \subseteq U^{B} \times X^{(\mathbb{N} \backslash B)}$.

Definition 6.3. For any $A \subseteq \mathbb{N}$, define a group topology $\mathfrak{t}_{A}$ on $c_{0}(X)$ having as a neighborhood basis at zero the family of sets $(P(B, U))$, where $U$ runs through all neighborhoods of zero in $X$ and $B$ through all elements of $\mathscr{P}(\mathbb{N})$ with finite $A \triangle B$.

Obviously, $\mathfrak{t}_{\varnothing}=\mathfrak{p}_{0}$ and $\mathfrak{t}_{\mathbb{N}}=\mathfrak{u}_{0} ;$ more precisely, $\mathfrak{t}_{A}=\mathfrak{p}_{0}\left(\mathfrak{t}_{A}=\mathfrak{u}_{0}\right)$ if and only if $A$ is finite (resp., co-finite). 
Lemma 6.4. If $P(A, U) \in \mathfrak{t}_{B}$ with $U \neq X$, then $A \backslash B$ is finite. Consequently, $A \backslash B$ is finite whenever $\mathfrak{t}_{A} \leq \mathfrak{t}_{B}$.

Proof. By our hypothesis, there exists a subset $B^{\prime}$ of $\mathbb{N}$, such that $B^{\prime} \Delta B$ is finite and $P\left(B^{\prime}, U^{\prime}\right) \subseteq P(A, U)$ for some neighborhood $U^{\prime}$ of 0 in $X$. Then, by Item (b) of Notation 6.2, $A \subseteq B^{\prime}$. Since $\left|B^{\prime} \Delta B\right|<\infty$, this proves that $A \backslash B$ is finite, as well.

Now, assume that $\mathfrak{t}_{A} \leq \mathfrak{t}_{B}$. Then, $P(A, U) \in \mathfrak{t}_{A}$, so $P(A, U) \in \mathfrak{t}_{B}$, as well. Hence, $A \backslash B$ is finite by the fist part of the argument.

Proposition 6.5. For the topological group $\left(G, \mathfrak{u}_{0}\right)=\left(c_{0}(X), \mathfrak{u}_{0}\right)$, the mapping:

$$
\mathscr{P}(\mathbb{N}) \longrightarrow \mathscr{C}(G), A \longmapsto \mathfrak{t}_{A}
$$

is order preserving. Moreover, $\mathfrak{t}_{A}=\mathfrak{t}_{B}$ for $A, B \in \mathscr{P}(\mathbb{N})$ if and only if the symmetric difference $A \Delta B$ is finite.

Proof. The mapping (7) is well defined, since for any $A \subseteq \mathbb{N}$, we have $\mathfrak{p}_{0} \subseteq \mathfrak{t}_{A} \subseteq \mathfrak{u}_{0}$. Since all of the topologies $\mathfrak{t}_{A}$ are also locally quasi-convex, they belong to $\mathscr{C}(G)$.

Assume that $\mathfrak{t}_{A}=\mathfrak{t}_{B}$. Applying twice the second assertion in Lemma 6.4, we conclude that $A \Delta B$ is finite.

Define an equivalence relation on $\mathscr{P}(\mathbb{N})$ by letting $A \sim B$ for $A, B \in \mathscr{P}(\mathbb{N})$ whenever $|A \Delta B|<\infty$. Denote the set of equivalence classes by $\mathscr{P}(\mathbb{N})_{*}$ and its elements by $A_{*}$; moreover, write $A \subseteq{ }^{*} B\left(A={ }^{*} B\right)$ for subsets of $\mathbb{N}$ when $|A \backslash B|<\infty$ (resp., $A \Delta B$ ) is finite. In these terms, Proposition 6.5 can be reformulated as follows:

Corollary 6.6. The mapping $\mathscr{P}(\mathbb{N})_{*} \rightarrow \mathscr{C}(G), A_{*} \mapsto \mathfrak{t}_{A}$ is a poset embedding.

Remark 6.7. The above Proposition 6.5 implies that $\sup \left\{\mathfrak{t}_{A}, \mathfrak{t}_{B}\right\} \leq \mathfrak{t}_{A \cup B}$. However, one can easily check with Notation 6.2(a) that actually, $\sup \left\{\mathfrak{t}_{A}, \mathfrak{t}_{B}\right\}=\mathfrak{t}_{A \cup B}$ holds true. In particular, if $P(A, U) \in \sup \left\{\mathfrak{t}_{B_{1}}, \ldots, \mathfrak{t}_{B_{n}}\right\}=\mathfrak{t}_{B_{1} \cup \ldots \cup B_{n}}$ with $U \neq X$, then $A \subseteq \subseteq^{*} \cup_{i} B_{i}$ by Lemma 6.4 .

The structure of $\left(\mathscr{P}(\mathbb{N})_{*}, \subseteq\right)$ is very rich, as the following example shows.

Example 6.8. There exists an anti-chain of size $\mathfrak{c}$ in $\left(\mathscr{P}(\mathbb{N})_{*}, \subseteq\right)$. Although this fact is well known (see, for example, [24]), we give a brief argument for the reader's convenience.

As the poset $\mathscr{P}(\mathbb{N})_{*}$ is isomorphic to $\mathscr{P}(\mathbb{Q})_{*}$, it is enough to check that width $\left(\mathscr{P}(\mathbb{Q})_{*}\right)=\mathfrak{c}$. For every $\rho \in \mathbb{R}$, pick a one-to-one sequence of rational numbers $\left(r_{n}^{\rho}\right)$ converging to $\rho$. Then, the sets $A_{\rho}:=\left\{r_{n}^{\rho}: n \in \mathbb{N}\right\}, \rho \in \mathbb{R}$, form an almost disjoint family witnessing width $\left(\mathscr{P}(\mathbb{Q})_{*}\right)=\mathfrak{c}$.

All topologies of the form $\mathfrak{t}_{A}$ produced so far, including the top and the bottom element $\left(\mathfrak{u}_{0}\right.$ and $\mathfrak{p}_{0}$, resp.) of $\mathscr{C}(G)$, are metrizable (so, second countable, since $G$ 
is separable by (3.4) in [23]). This makes it natural to ask whether all compatible topologies are metrizable. The next theorem and Example 6.10 answer this question negatively in the strongest possible way.

Theorem 6.9. The poset $\mathscr{C}(G)$ is quasi-isomorphic to $\mathscr{P}(\mathbb{R})$, so $|\mathscr{C}(G)|=2^{\mathfrak{c}}$ and $\mathscr{C}(G)$ has chains of length $\mathfrak{c}^{+}$.

Proof. According to Proposition 3.1, $\mathscr{C}(G)$ embeds into $\mathscr{P}(\mathbb{R})$ as $\left(G, \mathfrak{u}_{0}\right)$ is Mackey and $\left|\mathfrak{u}_{0}\right|=\mathfrak{c}$. Therefore, it suffices to show that $\mathscr{P}(\mathbb{R})$ embeds into $\mathscr{C}(G)$. It is enough to produce an embedding of $\mathscr{P}(\mathbb{R}) \backslash\{\varnothing\}$ into $\mathscr{C}(G) \backslash\left\{\mathfrak{p}_{0}\right\}$. To this end, we shall replace $\mathbb{R}$ by an almost disjoint family $\mathfrak{A}$ of size $\mathfrak{c}$ of infinite subsets of $\mathbb{N}$ (see Example 6.8).

For $\varnothing \neq \mathcal{B} \subseteq \mathfrak{A}$, let $\mathcal{T}_{\mathcal{B}}=\sup \left\{\mathfrak{t}_{A}: A \in \mathcal{B}\right\}$. It suffices to prove that the mapping:

$$
\mathscr{P}(\mathfrak{A}) \backslash\{\varnothing\} \longrightarrow \mathscr{C}(G), \mathcal{B} \longmapsto \mathcal{T}_{\mathcal{B}}
$$

is injective. To this end, it suffices to show that $\mathcal{T}_{\mathcal{B}} \leq \mathcal{T}_{\mathcal{B}^{\prime}}$ implies $\mathcal{B} \subseteq \mathcal{B}^{\prime}$ for $\varnothing \neq \mathcal{B}, \mathcal{B}^{\prime} \in \mathscr{P}(\mathfrak{A})$. Pick $A \in \mathcal{B}$ and fix a neighborhood $U \subsetneq X$ of zero. As $P(A, U) \in \mathfrak{t}_{A} \subseteq \mathcal{T}_{\mathcal{B}} \subseteq \mathcal{T}_{\mathcal{B}^{\prime}}$, there exists a finite subset $\mathcal{B}_{0}^{\prime} \subseteq \mathcal{B}^{\prime}$, such that $P(A, U) \in$ $\mathcal{T}_{\mathcal{B}_{0}^{\prime}}=\mathfrak{t}_{\cup} \mathcal{B}_{0}^{\prime}$. Then, $A \subseteq^{*} \cup \mathcal{B}_{0}^{\prime}$ by Remark 6.7. Since the finite set $\{A\} \cup \mathcal{B}_{0}^{\prime}$ is a subfamily of the almost disjoint family $\mathfrak{A}$, this may occur only if $A=B$ for some member $B \in \mathcal{B}^{\prime}$, i.e., $A \in \mathcal{B}_{0}^{\prime} \subseteq \mathcal{B}^{\prime}$.

For the last assertion, we need the following notation. For infinite cardinals $\kappa, \lambda$, we shall write $C(\kappa, \lambda)$ if, for a set of size $\kappa$, the poset $\mathscr{P}(\kappa)$ has a chain of size $\lambda$ (for more details, see $[7,25])$. In these terms, Sierpinksi has proven that $C\left(2^{<\lambda}, 2^{\lambda}\right)$ holds true for every infinite cardinal $\lambda$, where $2^{<\lambda}=\sup \left\{2^{\mu}: \mu<\lambda\right\}$. Now, let $\lambda$ be the minimal cardinal, such that $2^{\lambda}>\mathfrak{c}$. Then, obviously, $2^{<\lambda}=\mathfrak{c}$, since $\lambda>\omega$. As $2^{\lambda} \geq \mathfrak{c}^{+}, C\left(2^{<\lambda}, 2^{\lambda}\right)$ implies that $C\left(\mathfrak{c}, \mathfrak{c}^{+}\right)$holds true (see also [7] (Corollary 1.6) for the proof of $C\left(\kappa, \kappa^{+}\right)$for arbitrary $\left.\kappa\right)$. In other words, $\mathscr{P}(\mathbb{R})$ admits chains of length $\mathfrak{c}^{+}$.

By Proposition 3.1, the set of metrizable group topologies in $\mathscr{C}(G)$ has cardinality $\leq \mathfrak{c}^{\omega}=\mathfrak{c}$. Hence, the above theorem provides $2^{\mathfrak{c}}$ many non-metrizable topologies in $\mathscr{C}(G)$. For the sake of completeness, we provide a short intrinsic proof of this fact.

Example 6.10. Let $\mathfrak{A}$ be as in the above proof. We show that $\mathcal{T}_{\mathcal{B}}$ is not metrizable whenever $\mathcal{B}$ is an uncountable subset of $\mathfrak{A}$. Since there are $2^{\mathfrak{c}}$ many such sets, this provides $2^{\mathfrak{C}}$ many non-metrizable topologies in $\mathscr{C}(G)$. 
Assume that $\mathcal{T}_{\mathcal{B}}$ is metrizable, and let $\left(W_{k}\right)_{k \in \mathbb{N}}$ be a countable neighborhood basis at zero in $\mathcal{T}_{\mathcal{B}}$. For every $k$, there exists a finite subset $\mathcal{B}_{k}$ of $\mathcal{B}$, such that $W_{k} \in \mathcal{T}_{\mathcal{B}_{k}}$. Hence, there exist a neighborhood $U_{k}$ of zero in $X$ and $C_{k} \subseteq \mathbb{N}$ with:

$$
C_{k}={ }^{*} \bigcup \mathcal{B}_{k} \quad \text { and } \quad P\left(C_{k}, U_{k}\right) \subseteq W_{k} .
$$

Since $\mathcal{B}$ is uncountable, we can choose $A_{0} \in \mathcal{B} \backslash \bigcup_{k \in \mathbb{N}} \mathcal{B}_{k}$. Let $U \subsetneq X$ be a neighborhood of zero. Then, $P\left(A_{0}, U\right) \in \mathfrak{t}_{A_{0}} \subseteq \mathcal{T}_{\mathcal{B}}$. Therefore, $W_{k} \subseteq P\left(A_{0}, U\right)$ for some $k \in \mathbb{N}$. Now, the inclusion in (8) yields $P\left(C_{k}, U_{k}\right) \subseteq P\left(A_{0}, U\right)$. Hence, Notation 6.2(b) implies $A_{0} \subseteq C_{k}={ }^{*} \cup \mathcal{B}_{k}$. Since these are finitely many members of an almost disjoint family, we conclude that $A_{0} \in \mathcal{B}_{k}$, a contradiction.

\section{Final Comments and Open Questions}

The embedding $\mathfrak{F}_{\omega} \hookrightarrow \mathscr{C}(G)$ remains true for many non-compact $\sigma$-compact LCA groups $G$. Actually, the $\sigma$-compact LCA groups $G$ for which our approach does not ensure an embedding of $\mathfrak{F}_{\omega}$ in $\mathscr{C}(G)$ must be non-r-disconnected. Hence, they must have a very special structure (namely, contain a compact subgroup $K$, such that $G / K \cong \mathbb{R}^{n} \times L \times \prod_{i=1}^{k} \mathbb{Z}\left(p_{i}^{\infty}\right)$ for some $n, m, k \in \mathbb{N}$, a discrete torsion-free non-r-discrete group $L$ and not necessarily distinct primes $p_{i}$, see Example 2.6). Still simpler is this structure in the case when $G$ is supposed additionally to have a compact open subgroup (this eliminates the vector subgroup $\mathbb{R}^{n}$ ). Indeed, in this case, $G$ must contain an open subgroup of the form $K \times \mathbb{Z}^{m}$, for some $m \in \mathbb{N}$ and a compact group $K$, so that $G /\left(K \times \mathbb{Z}^{m}\right) \cong \prod_{i=1}^{k} \mathbb{Z}\left(p_{i}^{\infty}\right)$ for some $k \in \mathbb{N}$ and not necessarily distinct primes $p_{i}$.

In the general case, we have proved in [16] that $|\mathscr{C}(G)| \geq 3$ for every non-compact LCA group $G$. In particular, $|\mathscr{C}(\mathbb{R})| \geq 3,|\mathscr{C}(\mathbb{Z})| \geq 3$ and $\mid \mathscr{C}\left(\mathbb{Z}\left(\left(p^{\infty}\right)\right) \mid \geq 3\right.$ for every prime $p$. Further progress in this direction depends pretty much on the following:

Questions 7.1. (a) Compute $|\mathscr{C}(\mathbb{Z})|$. Is it infinite? Is it countable? Is it at most $\mathfrak{c}$ ? At least $\mathfrak{c}$ ?

(b) Compute $|\mathscr{C}(\mathbb{R})|$. Is it infinite? Is it countable? Is it at most $\mathfrak{c}$ ? At least $\mathfrak{c}$ ?

(c) Compute $\left|\mathscr{C}\left(\mathbb{Z}\left(p^{\infty}\right)\right)\right|$, where $p$ is a prime. Is it infinite? Is it countable? Is it at most $\mathfrak{c}$ ? At least $\mathfrak{c}$ ?

Note that in (a) and (c), the group in question is countable, so that $2^{c}$ is an obvious upper bound in both cases.

Any information in the direction of Item (c) will throw light on the poset $\mathscr{C}\left(\mathbb{Q}_{\pi}\right)$ for all $\pi$ containing $p$ (as $\mathbb{Q}_{\pi}$ has a quotient isomorphic to $\mathbb{Z}\left(p^{\infty}\right)$, so the poset $\mathscr{C}\left(\mathbb{Z}\left(p^{\infty}\right)\right.$ embeds into the poset $\mathscr{C}\left(\mathbb{Q}_{\pi}\right)$ by Theorem 3.10). 
Clearly, $\mathrm{CH}$ is needed in Corollary 1.12 only to eliminate those groups $\mathrm{G}$ that have $\omega<\varrho(G)<\mathfrak{c}$. We do not know if the assertion from this corollary remains true without the assumption of $\mathrm{CH}$. In particular, the following question remains open:

Question 7.2. Is $\mathscr{C}\left(\mathbb{R} \oplus \bigoplus_{\omega_{1}} \mathbb{Z}_{2}\right) \stackrel{\text { q.i. }}{\cong} \mathfrak{F} \omega_{1}$ ?

Question 7.3. Let $G$ be a non-precompact second countable Mackey group. Is it true that $|\mathscr{C}(G)| \geq \mathfrak{c}$ ?

Here comes a somewhat general question:

Problem 7.4. Find sufficient conditions for a metrizable precompact group $G$ to be Mackey (i.e., have $|\mathscr{C}(G)|=1$ ).

Such a sufficient condition was pointed out in [3]: all bounded metrizable precompact groups are Mackey.

The next question is related to Question 7.3:

Question 7.5. If for a group $G$, one has $|\mathscr{C}(G)|>1$, can $\mathscr{C}(G)$ be finite? In particular, can one have a Mackey group $G$ with $|\mathscr{C}(G)|=2$ ?

The following conjecture will positively answer Question 7.3 (as well as Question 7.1) and negatively answer Question 7.5:

Conjecture 7.6. [Mackey dichotomy] For a locally quasi-convex group $G$, one has either $|\mathscr{C}(G)|=1$ or $|\mathscr{C}(G)| \geq \mathfrak{c}$.

Positive evidence in the case of bounded groups can be obtained from the recent results in [26].

Let $\lambda$ be a non-discrete linear topology on $\mathbb{Z}$. It is easy to see that $(\mathbb{Z}, \lambda)$ is metrizable and precompact. It is shown in [27] that these groups are not Mackey, i.e., $|\mathscr{C}(\mathbb{Z}, \lambda)|>1$.

Question 7.7. How large can $\mathscr{C}(\mathbb{Z}, \lambda)$ be?

Let $(G, \tau)$ be infinite torsion subgroup of $\mathbb{T}$ equipped with the (precompact metrizable) topology $\tau$ induced by $\mathbb{T}$. It was proven in [28] that $(G, \tau)$ is not a Mackey group, i.e., $|\mathscr{C}(G, \tau)|>1$.

Question 7.8. How large can be $\mathscr{C}(G, \tau)$ in this case?

Acknowledgments: Dikran Dikranjan was partially supported by Fondazione Cassa di Risparmio di Padova e Rovigo (Progetto di Eccellenza "Algebraic structures and their applications"). Elena Martín-Peinador was partially supported by the Spanish Ministerio de Economía y Competitividad. Project: MTM 2013-42486-P.

Author Contributions: Lydia Außenhofer, Dikran Dikranjan and Elena Martín-Peinador contributed equally to this work. 
Conflicts of Interest: The authors declare no conflict of interest.

\section{References}

1. Varopoulos, N.T. Studies in harmonic analysis. Proc. Camb. Phil. Soc. 1964, 60, 467-516.

2. Chasco, M.J.; Martín-Peinador, E.; Tarieladze, V. On Mackey topology for groups. Stud. Math. 1999, 132, 257-284.

3. De Leo, L. Weak and Strong Topologies in Topological Abelian Groups. Ph.D. Thesis, Universidad Complutense de Madrid, Madrid, Spain, July 2008.

4. De Leo, L.; Dikranjan, D.; Martín-Peinador, E.; Tarieladze, V. Duality Theory for Groups Revisited: g-barrelled groups, Mackey \& Arens Groups. 2015, in preparation.

5. Bonales, G.; Trigos-Arrieta, F.J.; Mendoza, R.V. A Mackey-Arens theorem for topological Abelian groups. Bol. Soc. Mat. Mex. III 2003, 9, 79-88.

6. Berarducci, A.; Dikranjan, D.; Forti, M.; Watson, S. Cardinal invariants and independence results in the lattice of precompact group topologies. J. Pure Appl. 1998, 126, $19-49$.

7. Comfort, W.; Remus, D. Long chains of Hausdorff topological group topologies. J. Pure Appl. Algebra 1991, 70, 53-72.

8. Comfort, W.; Remus, D. Long chains of topological group topologies-A continuation. Topology Appl. 1997, 75, 51-79.

9. Dikranjan, D. The Lattice of Compact Representations of an infinite group. In Proceedings of Groups 93, Galway/St Andrews Conference, London Math. Soc. Lecture Notes 211; Cambidge Univ. Press: Cambridge, UK, 1995; pp. 138-155.

10. Dikranjan, D. On the poset of precompact group topologies. In Topology with Applications, Proceedings of the 1993 Szekszàrd (Hungary) Conference, Bolyai Society Mathematical Studies; Czászár, Á., Ed.; Elsevier: Amsterdam, The Netherlands, 1995; Volume 4, pp. 135-149.

11. Dikranjan, D. Chains of pseudocompact group topologies. J. Pure Appl. Algebra 1998, 124, 65-100.

12. Engelking, R. General Topology, (Sigma Series in Pure Mathematics, 6), 2nd ed.; Heldermann Verlag: Berlin, Germany, 1989.

13. Abramsky, S.; Jung, A. Domain theory. In Handbook of Logic in Computer Science III; Abramsky, S., Gabbay, D.M., Maibaum, T.S.E., Eds.; Oxford University Press: New York, NY, USA, 1994; pp. 1-168.

14. Banaszczyk, W. Additive Subgroups of Topological Vector Spaces, Lecture Notes in Mathematics; Springer Verlag: Berlin, Germany, 1991; Volume 1466.

15. Enflo, P. Uniform structures and square roots in topological groups. Israel J. Math. 1970, 8, 230-252.

16. Außenhofer, L.; Dikranjan, D.; Martín-Peinador, E. Locally quasi-convex compatible topologies on $\sigma$-compact LCA groups. 2015, in preparation.

17. Fuchs, L. Infinite Abelian Groups; Academic Press: New York, NY, USA, 1970.

18. Dikranjan, D.; Shakhmatov, D. Topological groups with many small subgroups. Topology Appl. 2015, in press. 
19. Dikranjan, D.; Prodanov, I.; Stojanov, L. Topological Groups (Characters, Dualities, and Minimal Group Topologies); Marcel Dekker, Inc.: New York, NY, USA, 1990.

20. Bruguera, M.; Martín-Peinador, E. Open subgroups, compact subgroups and Binz-Butzmann reflexivity. Topology Appl. 1996, 72, 101-111.

21. Außenhofer, L. A note on weakly compact subgroups of locally quasi-convex groups. Arch. Math. 2013, 101, 531-540.

22. Dikranjan, D.; Protasov, I. Counting maximal topologies on countable groups and rings. Topology Appl. 2008, 156, 322-325.

23. Dikranjan, D.; Martín-Peinador, E.; Tarieladze, V. Group valued null sequences and metrizable non-Mackey groups. Forum Math. 2014, 26, 723-757.

24. Sierpinski, W. Cardinal and ordinal numbers; Panstwowe Wydawnictwo Naukowe: Warsaw, Poland, 1958.

25. Baumgartner, J.E. Almost disjoint sets, the dense set problem and the partition calculus. Ann. Math. Logic 1976, 10, 401-439.

26. De la Barrera Mayoral, D.; Dikranjan, D.; Martìn Peinador, E. “Varopoulos paradigm": Mackey property $v s$. metrizability in topological groups. 2015, in preparation.

27. Außenhofer, L.; de la Barrera Mayoral, D. Linear topologies on $\mathbb{Z}$ are not Mackey topologies. J. Pure Appl. Algebra 2012, 216, 1340-1347.

28. De la Barrera Mayoral, D. $\mathbb{Q}$ is not Mackey group. Topology Appl. 2014, 178, 265-275. 


\title{
Open and Dense Topological Transitivity of Extensions by Non-Compact Fiber of Hyperbolic Systems: A Review
}

\section{Viorel Nitica and Andrei Török}

\begin{abstract}
Currently, there is great renewed interest in proving the topological transitivity of various classes of continuous dynamical systems. Even though this is one of the most basic dynamical properties that can be investigated, the tools used by various authors are quite diverse and are strongly related to the class of dynamical systems under consideration. The goal of this review article is to present the state of the art for the class of Hölder extensions of hyperbolic systems with non-compact connected Lie group fiber. The hyperbolic systems we consider are mostly discrete time. In particular, we address the stability and genericity of topological transitivity in large classes of such transformations. The paper lists several open problems and conjectures and tries to place this topic of research in the general context of hyperbolic and topological dynamics.
\end{abstract}

Reprinted from Axioms. Cite as: Nitica, V.; Török, A. Open and Dense Topological Transitivity of Extensions by Non-Compact Fiber of Hyperbolic Systems: A Review. Axioms 2016, 4, 84-101.

\section{Introduction}

A dynamical system is a continuous map $f$ of a topological space $X$. We emphasize that in this paper, $X$ will be mostly a non-compact set and $f$ will be invertible. Given a dynamical system $(X, f)$, a basic property that one may study is topological transitivity, that is the existence of a dense forward orbit $x, f(x), f^{2}(x), \ldots$ If $X$ is locally compact separable without isolated points, then $(X, f)$ is topologically transitive if and only if for any non-empty open subsets $U, V \subset X$, there exists $n \geq 1$, such that $f^{n}(U) \cap V \neq \varnothing$. The topological spaces that we will work with, Riemannian manifolds and phase spaces of shifts of the finite type, satisfy these conditions. Let us observe that other, sometimes equivalent with ours, definitions are introduced in the literature for topological transitivity. One may refer to the survey papers of Blanchard [1] or Kolyada-Snoha [2] for a more in-depth discussion and other definitions/interpretations of topological transitivity. This notion is also closely related to the notion of topological chaos introduced by Devaney [3]. The original definition of topological chaos given by Devaney, in addition to topological transitivity, requires the existence of a dense set of periodic points and the sensitive dependence of initial data for the dynamical system. It was 
later shown by Banks et al. [4] that the sensitivity of the initial data is a consequence of the other two conditions.

Various examples of topological transitive transformations are constructed in the literature. In some respects, topological transitivity is the topological counterpart of ergodicity. We recall that a probability measure $\mu$ on a measurable space $X$ is ergodic with respect to a measurable map $f: X \rightarrow X$ if and only if the only $f$-invariant subsets, up to subsets of measure zero, are $X$ and the empty set. If $X$ is compact topological space and the continuous map $f$ has an invariant Borel probability measure $\mu$, which is positive on open sets, then topological transitivity is implied by ergodicity. It was shown by Oxtoby and Ulam [5] that ergodicity is a residual property, in the set of homeomorphisms of a manifold $X$ of dimension at least two, if $\mu$ is a nonatomic measure of full support with $\mu(\partial X)=0$. We recall that a residual property for a complete metric space is one that is valid for a second Baire category subset.

Examples of topological transitive transformations of the plane are constructed by Besicovitch [6,7] and Shnirelman [8]. Their work was generalized by Sidorov [9], who constructed topological transitive extensions of a topologically transitive map with fiber an arbitrary Banach space.

The class of hyperbolic dynamical systems, introduced in the 1960s-1970s by Anosov and Sinai [10] in the USSR and by Bowen [11] and Smale [12] in the USA, provides many examples of ergodic and, in particular, topologically transitive transformations. Hyperbolic systems have a splitting of the tangent bundle into two invariant subbundles, one contracting and one expanding. These bundles are integrable into stable/unstable foliations. A standard reference for the theory of hyperbolic dynamical systems is the monograph of Katok and Hasselblatt [13]. During our exposition, we will assume as known or already defined many standard notions discussed there. Similar techniques can be applied to continuous dynamical systems, such as hyperbolic flows. Building on an argument of Hopf [14], who proved the ergodicity of the geodesic flow of a surface of negative curvature, Anosov [15] proved the ergodicity of the geodesic flow of any manifold of negative curvature. A key ingredient of the proof is the existence of invariant stable and unstable foliations for the geodesic flow. These foliations are, in general, only transversally Hölder, but exhibit the absolute continuity of the holonomy maps; this allows the Hopf argument to be carried out.

Partially hyperbolic diffeomorphisms were introduced in the 1980s by Brin and Pesin [16] as a generalization of hyperbolic diffeomorphisms. Partially hyperbolic diffeomorphisms have a splitting of the tangent bundle into three invariant subbundles, one contracting, one expanding and one, called the center bundle, for which the expansion/contraction is in-between. The contracting and expanding subbundles are always integrable into stable/unstable foliations. It was expected that, 
in the presence of a smooth invariant volume, ergodicity with respect to the volume measure should be true for many of these transformations. Brin and Pesin [16] proved ergodicity under the assumptions that the stable/unstable foliations are smooth and form an accessible pair, that is, one can travel from any point in the manifold to any other point in the manifold via a path built out of segments sitting inside stable/unstable leaves. The argument was carried forward by Grayson, Pugh and Shub [17], Pugh and Shub [18] and Burns and Wilkinson [19]. The state of the art in this direction is that the accessibility of the pair of stable/unstable foliations implies ergodicity. Nevertheless, accessibility turned out to be difficult to prove. Some progress was done in the case of a one-dimensional center subbundle; see, e.g., [20]. Hertz [21] proved the stable ergodicity of certain linear automorphisms of the torus. Furthermore, Dolgopyat and Wilkinson [22] proved that stable accessibility is dense in the $C^{1}$-topology for the class of volume preserving partially hyperbolic diffeomorphisms. Pugh and Shub conjectured that in $C^{2}$ and even $C^{r}, r \geq 2$ topology, accessibility is open and dense.

If the invariant volume does not exist, then even in the presence of partial hyperbolicity, different tools are needed in order to study topological transitivity. Given a dynamical system $(X, f)$, a point $x \in X$ is called recurrent if for any neighborhood $U$ of $x$, there exists a positive integer $n \geq 1$, such that $f^{n}(x) \in U$. In particular, any periodic point is recurrent. Brin [23] proved that a $C^{1}$ diffeomorphism that has an accessible pair of stable/unstable foliations and a dense set of recurrent points is topologically transitive. This result has, nevertheless, limited applicability due to the difficulty of proving the accessibility and density of recurrent points. In particular, it is difficult to exhibit a dense set of periodic points for such transformations. Open sets of transitive partially hyperbolic diffeomorphisms are found by Bonatti and Diaz [24], building on previous examples found by Shub [25].

A robust obstruction to topological transitivity is the existence of a trapping region, i.e., a non-empty open proper subset $U \subset M$, such that $f(\bar{U}) \subseteq U$. When this obstruction does not occur, it follows from the work of Bonatti and Crovisier [26] that a generic $C^{1}$ diffeomorphism of a compact Riemannian manifold is topologically transitive. This result relies on the Pugh-Hayashi [27-29] closing lemma, and it is not available beyond the $C^{1}$ category.

Another direction currently pursued in the literature is that of linear topological chaos, that is, the study of topological chaos for infinite dimensional continuous linear operators [30]. The techniques employed in linear topological chaos are quite different from those employed in the study of hyperbolic dynamical systems and will not be discussed in this review.

In the rest of this review paper, we summarize the results about the topological transitivity for various classes of non-compact Lie group extensions of hyperbolic 
systems. These classes of dynamical systems can be thought of as thin classes of partially hyperbolic systems.

\section{Lie Group Extensions of Hyperbolic Systems: Basic Definitions and the Main Conjecture}

Definition 1. Consider a continuous transformation $f: X \rightarrow X$, a Lie group $\Gamma$ and a continuous map $\beta: X \rightarrow \Gamma$ called a cocycle. These determine a skew product, or $\Gamma$-extension,

$$
f_{\beta}: X \times \Gamma \rightarrow X \times \Gamma, \quad f_{\beta}(x, \gamma)=(f x, \gamma \beta(x))
$$

It is assumed throughout the rest of the paper that $X$ is a hyperbolic basic set. Of interest to us is whether non-compact Lie group extensions of a hyperbolic basic set are typically topologically transitive.

Definition 2. Let $\left(M, d_{M}\right)$ be a smooth manifold endowed with a Riemannian metric. Let $f: M \rightarrow M$ be a smooth diffeomorphism and $X \subset M$ a compact and $f$-invariant subset of $M$. We say that $X$ is hyperbolic if there exists a continuous $D f$-invariant splitting $E^{\mathcal{S}} \oplus E^{u}$ of the tangent bundle $T_{X} M$ and constants $C_{1}>0,0<\lambda<1$, such that for all $n \geq 0$ and $x \in X$, we have:

$$
\begin{gathered}
\left\|\left(D f^{n}\right)_{x} v\right\| \leq C_{1} \lambda^{n}\|v\|, v \in E_{x}^{s} \\
\left\|\left(D f^{-n}\right)_{x} v\right\| \leq C_{1} \lambda^{n}\|v\|, v \in E_{x}^{u}
\end{gathered}
$$

If $X$ coincides with $M$, then $f$ is called hyperbolic, or Anosov, diffeomorphism.

We say that $X$ is locally maximal if there exists an open neighborhood $U$ of $X$, such that every compact $f$-invariant set of $U$ is contained in $X$. A locally maximal hyperbolic set $X$ is a basic set for $f: M \rightarrow M$ if $f: X \rightarrow X$ is transitive and $X$ does not consist of a single periodic orbit.

We present some motivation for the study of Lie group extensions of hyperbolic systems. These transformations have many common properties with partially hyperbolic diffeomorphisms. If the fiber is a compact connected Lie group, the cocycle $\beta$ is at least $C^{1}$ and the hyperbolic basic set $(X, f)$ is an Anosov diffeomorphism, then the extension is a partially hyperbolic diffeomorphism. In general, we will see that if a certain bunching condition for the center direction holds, then $f_{\beta}$ has stable and unstable foliations. If the Lie group $\Gamma$ is not compact, the extension $f_{\beta}$ acts on a space that does not support an invariant probability measure. Thus, the class of non-compact Lie group extensions can be considered as a test bed for the more general class of partially hyperbolic diffeomorphisms that do not have a nice invariant probability measure. 
Given a connected Lie group $\Gamma$ and a cocycle $\beta: X \rightarrow \Gamma$, we consider the $\Gamma$-extension $f_{\beta}: X \times \Gamma \rightarrow X \times \Gamma$. We say that the cocycle $\beta$ is topologically transitive (for brevity, transitive,) if the corresponding skew product $f_{\beta}$ is transitive.

Let $L \Gamma$ be the Lie algebra of $\Gamma$. We denote by $e_{\Gamma}$ the identity element of $\Gamma$. Let Ad denote the adjoint representation of $\Gamma$ on $L \Gamma$. Let $\|\cdot\|$ be a norm on $L \Gamma$. There is a metric $d$ on $\Gamma$ with the following properties (see Pollicott and Walkden [31]):

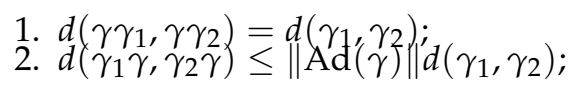

for any $\gamma, \gamma_{1}, \gamma_{2} \in \Gamma$.

Definition 3. Let $f: X \rightarrow X$ be a map and $\beta: X \rightarrow \Gamma$ a cocycle. For $k \geq 1$, we write $f_{\beta}^{k}(x, \gamma)=\left(f^{k} x, \gamma \beta(k, x)\right)$, where:

$$
\beta(k, x)=\beta(x) \beta(f x) \cdots \beta\left(f^{k-1} x\right)=\prod_{j=0}^{k-1} \beta\left(f^{j} x\right)
$$

(occasionally, we use the last formula to keep notation simple; its meaning is the ordered product given by the middle expression).

If $Q$ is a trajectory of $f$ of length $k$ (i.e., $Q=\left\{x, f(x), \ldots, f^{k-1}(x)\right\}$ for some $x$ ), then we define the height of $\beta$ over $Q$ to be $\beta(Q)=\beta(k, x)$. In particular, if $x$ is a periodic point of period $\ell$, then the height of the corresponding periodic orbit $P$ is $\beta(P)=\beta(\ell, x)$.

By abuse of notation, we often refer to "the periodic orbit $P$ " instead of "the orbit of the periodic point $x "$ when $x$ is clear from the context.

Definition 4. Given a cocycle $\beta: X \rightarrow \Gamma$ over $f: X \rightarrow X$, define $\mu \geq 1$ to be:

$$
\mu=\max \left\{\lim _{n \rightarrow \infty} \sup _{x \in X}\|\operatorname{Ad}(\beta(n, x))\|^{1 / n}, \lim _{n \rightarrow \infty} \sup _{x \in X}\left\|\operatorname{Ad}(\beta(n, x))^{-1}\right\|^{1 / n}\right\}
$$

For $f$ fixed, we say that the cocycle $\beta$ has subexponential growth if $\mu=1$.

Remark 1. The subexponential growth condition is automatically satisfied for any cocycle if the group $\Gamma$ is compact, nilpotent or a semidirect product of compact and nilpotent. This follows from the well-known result that nilpotent Lie groups have polynomial growth [32].

Recall the definition of cohomology: 
Definition 5. Let $\Gamma$ be a topological group. If $\beta_{1}, \beta_{2}: X \rightarrow \Gamma$ are continuous functions and $f: X \rightarrow X$ is a continuous map, then $\beta_{1}, \beta_{1}$ are called cohomologous (over $f$ ) if there exists a continuous map $u: X \rightarrow \Gamma$, such that:

$$
\beta_{1}=(u \circ f) \beta_{2} u^{-1}
$$

In [33], we proposed a general conjecture about topological transitivity in the class of Hölder cocycles. We start by observing that if the cocycle $\beta$ takes values in a proper closed sub-semigroup $S$ of the fiber $\Gamma$, then obviously $f_{\beta}$ is not transitive. An example is the group $\Gamma=\mathbb{R}$ with sub-semigroup $S$ consisting of the set of non-negative numbers. As Int $S \neq \varnothing$, we can construct open sets of nontransitive $\mathbb{R}$-extensions. Another example is the group $\Gamma=S L(n, \mathbb{R})$ with sub-semigroup $S$ consisting of matrices with non-negative entries. Since Int $S \neq \varnothing$, again we can construct open sets of nontransitive $S L(n, \mathbb{R})$-extensions.

Our conjecture is that this situation is the only essential obstruction to transitivity.

Conjecture 1 (Main Conjecture). Assume that $X$ is a hyperbolic basic set for $f: X \rightarrow X$ and $\Gamma$ is a finite-dimensional connected Lie group. Among the Hölder cocycles $\beta: X \rightarrow \Gamma$ with subexponential growth that are not cohomologous to a cocycle with values in a maximal sub-semigroup of $\Gamma$ with a non-empty interior, there is a Hölder open and dense set for which the extension $f_{\beta}$ is transitive.

\section{Statements of Available Results}

The conjecture is proven for various classes of Lie groups. The techniques used so far are quite diverse and seem to depend heavily on the particular properties of the group that appears in the fiber.

\section{1. $\Gamma$ Compact Connected Lie Group}

We start by observing that in this case, closed sub-semigroups coincide with closed subgroups and that there are no proper sub-semigroups with a nonempty interior. It was proven by Brin [23] that if the fiber $\Gamma$ is a compact connected Lie group, then topologically transitive extensions of a transitive Anosov diffeomorphism contain a set that is open and dense in the $C^{2}$-topology. An extension of Brin's general transitivity result is obtained in [34,35], in which accessibility is replaced by $\epsilon$-accessibility for any $\epsilon>0$. This improvement allows one to consider extensions with a disconnected base, such as subshifts of the finite type.

As observed in [20], Brin's result also holds in the Hölder topology. In fact, over an Anosov diffeomorphism, for any $r>0$, the $C^{r}$ cocycles that are transitive contain a Hölder-open (meaning $C^{s}$-open for any $s \in(0,1), s \leq r$ ) and $C^{r}$-dense 
set, and this result generalizes to extensions of a hyperbolic attractor. The latter result does not hold for extensions of general hyperbolic basic sets when $r<1$ (in particular, the result is false if $(X, f)$ is topologically conjugate to a subshift of finite type and $\Gamma$ is a torus: the interior of the transitive $C^{r}$-cocycles contains no cocycle of higher smoothness). However, for compact group extensions of general hyperbolic basic sets, Field et al. [36] proved that the transitive extensions contain a set that is: (i) Hölder open and dense (proving the Main Conjecture 1); and (ii) $C^{2}$-open, $C^{r}$-dense for all $r \geq 2$. See, also, [37-39].

Burns and Wilkinson [40] generalized Brin's result by showing the stability of the ergodicity of the extensions with compact fiber for perturbations in the class of $C^{r}$ diffeomorphisms.

\section{2. $\Gamma=\mathbb{R}^{n}$}

In this case, the maximal sub-semigroups with non-empty interior are the half-spaces whose bounding hyperplane contains the origin. Hence, stable transitivity is certainly not a generic property of $\mathbb{R}^{n}$-extensions. However, there are no further obstructions. We recall that a continuous map $f$ of a topological space $X$ is called weakly topologically mixing if $f \times f$ is topologically transitive. We can associate with each periodic orbit $f^{n} x=x$ a height $\beta(n, x):=\sum_{i=0}^{n-1} \beta\left(f^{i} x\right)$. We denote by $\mathrm{PD}_{\beta}=\left\{\beta(n, x): f^{n} x=x, x \in X\right\}$ the collection of all heights over the closed orbits of $f$.

Nitica and Pollicott [41] proved the following result:

Theorem 1. Let $X$ be an infranilmanifold, $f: X \rightarrow X$ an Anosov diffeomorphism and $\beta: X \rightarrow \mathbb{R}^{n}$ a Hölder cocycle. Then, the following are equivalent:

1. the cocycle $\beta$ is not cohomologous to a cocycle that takes values in a half-space;

2. the set $P D_{\beta}$ is not separated by any hyperplane passing through the origin;

3. the extension $f_{\beta}$ is transitive;

4. the extension $f_{\beta}$ is $C^{0}$-stably transitive;

5. the extension $f_{\beta}$ is weakly mixing;

6. the extension $f_{\beta}$ is $C^{0}$-stably weakly mixing;

7. for any direction in $\mathbb{R}^{n}$, there exist orbits of $f_{\beta}$ that are unbounded in the positive sense and orbits that are unbounded in the negative sense (i.e., $\forall v \in \mathbb{R}^{n}-\{0\}, \exists x, y \in X$, $\forall N>0, \exists n, m \geq 0$ such that $\langle\beta(n, x), v\rangle \geq N$ and $\langle\beta(m, y), v\rangle \leq-N)$.

Remark 2. We note that, due to a result of Bousch [42], one can check if a cocycle $\beta$ is cohomologous to one that takes values in a half-space by looking at the periodic data $P D_{\beta}$.

Therefore, one has a complete dichotomy for an $\mathbb{R}^{n}$-extension $f_{\beta}$ over an infranil Anosov diffeomorphism: either it is transitive (and, hence, stably transitive), or $\beta$ is cohomologous to a cocycle with values in such a half-space. Moreover, the transitive 
Hölder $\mathbb{R}^{n}$-extensions $f_{\beta}$ are actually $C^{0}$-stably transitive, that is, if $\beta^{\prime}$ is $C^{0}$-close enough to $\beta$ and $f_{\beta}$ is transitive, then $f_{\beta^{\prime}}$ is transitive.

A crucial ingredient in the proof of Theorem 1 is that the induced map in the first Čech cohomology groups $f^{*}: H^{1}(X, \mathbb{Z}) \rightarrow H^{1}(X, \mathbb{Z})$ does not have one as an eigenvalue. This is known to be the case for an Anosov diffeomorphism of an infranilmanifold, and it is an important open question as to whether this is the case for all Anosov diffeomorphisms (cf. [43]) (indeed, it is an open question as to whether there are Anosov diffeomorphisms on spaces other than infranilmanifolds).

Moss and Walkden [44] replace the condition about the induced map in cohomology by the weaker condition that the first Čech cohomology group $H^{1}(X, \mathbb{Z})$ has finite rank, where $X$ is a hyperbolic basic set. Moreover, the action in the base is extended to hyperbolic flows. Their proof gives an explicit and global description of the set of functions $\beta$ that give rise to transitive skew-products in terms of the cohomology of the hyperbolic basic set $X$.

We recall that a continuous map $f$ of a topological space $X$ is called topologically mixing if for any open subsets $U, V \subseteq X$, there exists a positive integer $N$, such that $f^{n}(U) \cap V \neq \varnothing$ for any $n \geq N$. The following problem seems to be open.

Problem 1. Find a topologically mixing $\mathbb{R}$-extension of an Anosov diffeomorphism.

For general hyperbolic basic sets, transitive $\mathbb{R}^{n}$-extensions need not be stably transitive. However, let $S$ denote the set of cocycles that are not cohomologous to a cocycle with values in a half-space. For cocycles in S, Field et al. [36] proved a result identical to that stated above for compact group extensions. Again this proves the Main Conjecture 1 for $\mathbb{R}^{n}$-extensions. Similar results hold for general Abelian finite-dimensional Lie groups $\Gamma=\mathbb{R}^{n} \times \mathbb{T}^{d}$, where $\mathbb{T}^{d}$ is a $d$-dimensional torus.

\section{3. $\Gamma$ Is a Euclidean-Type Group}

An important test case of a Euclidean-type group is the special Euclidean group, which is the semidirect product $\Gamma=S E(n)=S O(n) \ltimes \mathbb{R}^{n}$ with the action of $S O(n)$ on $\mathbb{R}^{n}$ given by the usual matrix multiplication. In this case, it is easy to see that there are no proper sub-semigroups with non-empty interior. It is shown in $[33,45,46]$ that when $n$ is even, the set of cocycles that are transitive is Hölder-open and $C^{r}$-dense, thus solving the conjecture in this case. The conjecture remains open for $n \geq 3$ odd. The difference between the case $n$ odd and $n$ even is due to the different behavior of a generic element in $\Gamma$ : if $n$ is even, then for a residual set of elements in $\Gamma$, the closure of the semigroup generated by an element is a compact subgroup of $\Gamma$; if $n$ is odd, then for a residual set of elements in $\Gamma$, the closure of the semigroup generated by an element is an unbounded subset of $\Gamma$. 
Problem 2. For $0<\alpha<1$, find a $C^{\alpha}$-stable transitive $S E(3)$-extension of an Anosov diffeomorphism.

In this direction, we show in [47] that for $S E(n)$-extensions, $n \geq 3$ odd, the transitivity is generic.

Theorem 2. Let $X$ be a basic hyperbolic set for $f: X \rightarrow X$. Let $r>0$, and let $n \geq 3$ be odd. Amongst the $C^{r}$ cocycles $\beta: X \rightarrow S E(n)$, the transitive cocycles form a residual set.

More generally, one may consider Euclidean-type groups of the form $\Gamma=$ $G \ltimes \mathbb{R}^{n}$, where $G$ is a compact connected Lie group acting linearly (and orthogonally) on $\mathbb{R}^{n}$, and the group multiplication is given by:

$$
\left(g_{1}, v_{1}\right)\left(g_{2}, v_{2}\right)=\left(g_{1} g_{2}, v_{1}+g_{1} v_{2}\right)
$$

Let Fix $G=\left\{v \in \mathbb{R}^{n}: g v=v\right.$ for all $\left.g \in G\right\}$. Set $\pi: \Gamma \rightarrow$ Fix $G$ to be the projection onto the $\mathbb{R}^{n}$-component and then orthogonal projection onto Fix $G$. If Fix $G \neq\{0\}$, then there is an obvious obstruction to transitivity, namely that $\pi \beta: X \rightarrow$ Fix $G$ takes values in a half-space. More generally, if $\pi \beta$ is cohomologous to a cocycle with values in a half space, then $f_{\beta}$ is not transitive. This is the only obstruction in generalizing Theorem 2 to general Euclidean-type groups.

Theorem 3. Let $X$ be a basic hyperbolic set for $f: X \rightarrow X$, and let $\Gamma=G \ltimes \mathbb{R}^{n}$ be a Euclidean-type group. Let $r>0$. Define $\mathcal{S}$ to be the space of $C^{r}$ cocycles $\beta: X \rightarrow \Gamma$ for which $\pi \beta: X \rightarrow$ Fix $G$ is not cohomologous to a cocycle with values in a half-space.

Then, $\mathcal{S}$ is an open subset of the space of $C^{r}$ cocycles, and the transitive cocycles $\beta: X \rightarrow \Gamma$ form a residual subset of $\mathcal{S}$.

Remark 3. (1) If Fix $G=0$, then there is no obstruction to transitivity, so Theorem 2 is a special case of Theorem 3 .

(2) By a standard argument, the set of transitive $C^{r}$ cocycles can be written as a countable intersection of $C^{r}$-open sets. We include the argument below. Hence, it suffices to prove the density in Theorems 2 and 3.

Choose a countable basis $\left\{U_{k}\right\}_{k}$ of the topology on $X \times \Gamma$ and denote by $C_{k, \ell}^{r}$ the $C^{r}$ cocycles $\beta \in \mathcal{S}$ for which there is a positive integer $n$, such that $f_{\beta}^{n}\left(U_{k}\right) \cap U_{\ell} \neq \varnothing$. Each set $C_{k, \ell}^{r}$ is clearly $C^{r}$-open, and $f$ is transitive if and only if $\beta$ is in each of the sets $C_{k, \ell}^{r}$. 


\section{4. Г Is a Nilpotent Lie Group}

Definition 6. For $n \geq 1$, let $\mathcal{H}_{n}$ denote the group consisting of matrices of the form:

$$
(a, b, c):=\left(\begin{array}{ccc}
1 & a^{T} & c \\
0 & I_{n} & b \\
0 & 0 & 1
\end{array}\right) \in \operatorname{Mat}_{n+2}(\mathbb{R})
$$

where $a, b \in \mathbb{R}^{n}, c \in \mathbb{R}$ and $I_{n}$ is the $n$-dimensional identity matrix.

Remark 4. (1) We can identify $\mathcal{H}_{n}$ with $\mathbb{R}^{n} \oplus \mathbb{R}^{n} \oplus \mathbb{R}$ endowed with the multiplication:

$$
(a, b, c)(A, B, C)=\left(a+A, b+B, c+C+a^{T} B\right)
$$

where $a, b, A, B \in \mathbb{R}^{n}, c, C \in \mathbb{R}$

(2) $\mathcal{H}_{1}$ is the standard three-dimensional Heisenberg group.

The center of $\mathcal{H}_{n}$ is $\left[\mathcal{H}_{n}, \mathcal{H}_{n}\right]=\{(0,0, c)\}=\mathbb{R}$. Denote $\widehat{\mathcal{H}}_{n}=\mathcal{H}_{n} / \mathbb{R} \cong \mathbb{R}^{2 n}$. If $\beta: X \rightarrow \mathcal{H}_{n}$ is a cocycle, denote by $\widehat{\beta}: X \rightarrow \widehat{\mathcal{H}}_{n}$ the corresponding quotient cocycle. There is an obvious obstruction to transitivity, namely that $\widehat{\beta}: X \rightarrow \widehat{\mathcal{H}}_{n} \cong \mathbb{R}^{2 n}$ takes values in a half-space bounded by a hyperplane passing through the origin (for brevity, call this a half-space from now on). More generally, if $\widehat{\beta}$ is cohomologous to a cocycle with values in a half-space, then $f_{\beta}$ is not transitive.

If $r>0$, let $\mathcal{S}^{r}\left(X, \mathcal{H}_{n}\right)$ be the set of $C^{r}$ cocycles $\beta: X \rightarrow \mathcal{H}_{n}$ for which $\widehat{\beta}$ is not cohomologous to a cocycle with values in a half-space. The main result in [48], improving on a weaker result showing only genericity and proven in [49], is:

Theorem 4 ([48, Theorem 1.4]). Assume that $X$ is a hyperbolic basic set for $f: X \rightarrow X$. Let $r>0$. Then $\mathcal{S}^{r}\left(X, \mathcal{H}_{n}\right)$ contains a dense and open set of transitive cocycles.

More precisely, we prove:

Theorem 5 ([48, Theorem 1.5]). Let $X$ be a hyperbolic basic set for $f: X \rightarrow X$ and $\beta: X \rightarrow \mathcal{H}_{n}$ a Hölder cocycle. If $\hat{\beta}: X \rightarrow \mathbb{R}^{2 n}$ is transitive, then so is $\beta$.

This implies Theorem 4, because, by [36,41]:

Theorem 6 ([48, Theorem 1.6]). For $r>0$, there is an open and dense set in $\mathcal{S}^{r}\left(X, \mathbb{R}^{d}\right)$ consisting of transitive cocycles.

A new technical tool needed in the proof of Theorem 4, which is of independent interest, is a diophantine approximation result, which shows the existence of an 
infinite set of approximate positive integer solutions for a diophantine system of equations consisting of a quadratic indefinite form and several linear equations, provided exact solutions exist over $\mathbb{R}$. The set of approximate solutions can be chosen to point in a certain direction; this direction can be chosen from a residual subset of full measure of the set of real directions solving exactly the system of equations.

Theorem 7 ([48, Theorem 6.2]). For $d \geq 2$, assume given in $\mathbb{R}^{d}$ a (homogeneous) quadratic form $Q$ and $k$ (homogeneous) linear forms $L_{1}, L_{2}, \ldots, L_{k}$, such that $\left.Q\right|_{\cap K e r} L_{i}$ is indefinite.

Assume that rankQ $\geq 2 k+3$. Then, for a residual, full measure set (in the induced topology/Lebesgue measure) of vectors $\mathbf{v} \neq \mathbf{0}$ in:

$$
\{Q=0\} \cap\left\{L_{i}=0,1 \leq i \leq k\right\}
$$

for any $\epsilon>0$, there are $\mathbf{x}_{n} \in \mathbb{Z}^{d}$, such that:

(1) $\left\|\mathbf{x}_{n}\right\| \rightarrow \infty$,

(2) $\sup \left|Q\left(\mathbf{x}_{n}\right)\right|<\infty$,

(3) $\operatorname{dist}\left(\mathbf{x}_{n}, \mathbb{R}_{+} \mathbf{v}\right) \leq \epsilon$.

In particular,

$$
\left|L_{i}\left(\mathbf{x}_{n}\right)\right| \leq C \epsilon, \text { for all } 1 \leq i \leq k \text { and all } n
$$

with a constant $C>0$ determined by the linear forms.

There is a class of nilpotent groups, analogous to the Heisenberg groups, but with a compact center, for which we obtain stronger results and for which the proofs are much simpler.

The normal subgroup of $\mathcal{H}_{n}$ generated by $(0,0,1)$ is isomorphic to $\mathbb{Z}$. Denote by $\Gamma_{n}$ the quotient $\mathcal{H}_{n} / \mathbb{Z}$. The center of $\Gamma_{n}$ is $\mathbb{R} / \mathbb{Z} \cong S^{1}$; let $\widehat{\Gamma}_{n}=\Gamma_{n} / S^{1} \cong \mathbb{R}^{2 n}$.

If $\beta: X \rightarrow \Gamma_{n}$ is a cocycle, denote by $\widehat{\beta}: X \rightarrow \mathbb{R}^{2 n}$ the corresponding quotient cocycle. For $r>0$, let $\mathcal{S}^{r}\left(X, \Gamma_{n}\right)$ be the set of $C^{r}$ cocycles $\beta: X \rightarrow \Gamma_{n}$ for which $\widehat{\beta}$ is not cohomologous to a cocycle with values in a half-space.

Theorem 8 ([49, Theorem 1.5]). Assume that $X$ is a basic hyperbolic set for $f: X \rightarrow X$. Let $n \geq 1, r>0$. Then, there is an open and dense set of transitive cocycles in $\mathcal{S}^{r}\left(X, \Gamma_{n}\right)$.

\section{5. $\Gamma$ Is a Compact and Nilpotent Semidirect Product}

In this subsection, we follow [50].

Definition 7. A connected Lie group $\Gamma$ is called a good semidirect product if it is a semidirect product $K \ltimes N$, where $N$ is a nilpotent Lie group, $K$ is a compact Lie group and, in addition, if $T$ is a maximal torus in $K$; the only element of $N$ fixed under conjugation by $T$ is the identity. 
Definition 8. A connected Lie group $\Gamma$ is perfect if its commutator subgroup $[\Gamma, \Gamma]$ coincides with $\Gamma$.

Theorem 9 ([50, Theorem 1.1]). Assume that $X$ is a hyperbolic basic set for $f: X \rightarrow X$. Let $\Gamma=K \ltimes N$ be a good semidirect product that is perfect. Then, in the class of $C^{r}$-cocycles $\beta: X \rightarrow \Gamma, r>0$, the transitive ones contain an open and dense set.

We describe next a large class of Lie groups that satisfy the assumptions in Theorem 9.

Definition 9. Let $\mathbb{K}$ be $\mathbb{R}$ or $\mathbb{C}$. Let $n$ be a positive integer, and let $n=n_{1}+n_{2}+\cdots+n_{\ell}$, where $n_{i}, 1 \leq i \leq \ell$ are also positive integers. Define $G\left(n_{1}, n_{2}, \ldots, n_{\ell}, \mathbb{K}\right)$ to be the subgroup of $G L(n, \mathbb{K})$, which consists of all block matrices:

$$
\left(\begin{array}{ccccc}
A_{1} & B_{1,2} & B_{1,3} & \ldots & B_{1, \ell} \\
0 & A_{2} & B_{2,3} & \ldots & B_{2, \ell} \\
0 & 0 & A_{3} & \ldots & B_{3, \ell} \\
\vdots & \vdots & \vdots & \ddots & \vdots \\
0 & 0 & 0 & \ldots & A_{\ell}
\end{array}\right)
$$

where:

- the block $A_{i}$ is an arbitrary matrix in the orthogonal group $S O\left(n_{i}\right)$ (respectively, the unitary group $\left.U\left(n_{i}\right)\right), 1 \leq i \leq \ell$;

- the block $B_{i, j}$ is an arbitrary matrix in Mat $\left(n_{i}, n_{j}\right)$, for $1 \leq i \leq \ell-1, i+1 \leq j \leq \ell$,

- the blocks below the diagonal are zero.

Remark 5. For $n \geq 3$, the partition $(n-1,1)$ gives the special Euclidean group $S E(n-1)$.

Theorem 10 ([50, Theorem 1.2]). Let $\mathbb{K}$ be $\mathbb{R}$ or $\mathbb{C}$. Let $n \geq 3$ be an integer and $n_{1}+n_{2}+$ $\cdots+n_{\ell}=n$ a partition of $n$, where $\left(n_{1}, n_{2}, \ldots, n_{\ell}\right)$ are even integers, all greater or equal to four, except possibly for a single occurrence of one. Then, $\Gamma=G\left(n_{1}, n_{2}, \ldots, n_{\ell}, \mathbb{K}\right)$ is a good semidirect product, which is perfect.

Problem 3. If $n_{i}=2$, for some $1 \leq i \leq \ell$, and if there is no more than one one among the $n_{i}$ 's, then the group $G\left(n_{1}, n_{2}, \ldots, n_{\ell}, \mathbb{R}\right)$ is a good semidirect product that is not perfect. If there are at least two ones among the $n_{i}$ 's, then the group $G\left(n_{1}, n_{2}, \ldots, n_{\ell}, \mathbb{R}\right)$ is not a good semidirect product. With the exception of the case $G=S E(2)=G(2,1, \mathbb{R})$, solved in [46] using results about extensions with Abelian fiber, Theorem 10 leaves open the transitivity conjecture for these cases. 
Definition 10. If $\Gamma$ is a topological group, we call $g \in \Gamma$ compact if the closure of the subgroup generated by $g$ is compact. We denote by $\mathcal{C}(\Gamma)$ the set of compact elements in $\Gamma$.

Remark 6. The proof of Theorem 9 is based on the existence of an open dense set of compact elements in $\Gamma$. It is shown in [51] that if a connected Lie subgroup $H$ of $G L(n, \mathbb{K})$ contains an open neighborhood of the identity in which compact elements are dense and is maximal with this property, then $H$ is conjugate to a group $G\left(n_{1}, n_{2}, \ldots, n_{\ell}, \mathbb{K}\right)$. This remark shows the optimality of our results and the limitations of the method. In order to solve Problem 3 , one needs to develop new techniques.

\section{6. Г Is a Non-Compact Semisimple Lie Group}

The conjecture is not verified for any non-compact semisimple Lie group. Nevertheless, for $\Gamma=S L(2, \mathbb{R})$ and, more generally, for $\Gamma=S p(n, \mathbb{R})$, open sets of transitive extensions are constructed in [33]. Here is a brief description of the construction. One uses the existence of compact elements in $\Gamma$ that are stably compact under small perturbations. No such elements are known in $S L(3, \mathbb{R})$ or many other non-compact semisimple Lie groups. These compact elements can be chosen arbitrarily close to the identity. We start with the identity cocycle $\beta$. After a small perturbation, we can arrange for the height of $\beta$ over the orbit of a fixed periodic point $x_{0} \in X$ to be a stably compact element. Using the method described in Section 4 , this allows one to construct a set of generators for $\Gamma$ in the range $\mathcal{L}_{\beta}\left(x_{0}\right)$ (defined in Section 4) that are close to the identity. The generating property is stable under small perturbations due to a classical result of Kuranishi [52]. In conjunction with Theorem 11 in Section 4 , this gives a stably transitive extension with fiber $\operatorname{Sp}(n, \mathbb{R})$.

The following problem is left open:

Problem 4. For $\alpha \in(0,1)$, find a $C^{\alpha}$-stable transitive $S L(3, \mathbb{R})$-extension of an Anosov diffeomorphism.

\section{Criterion for Transitivity}

In order to prove the topological transitivity of extensions with non-compact fiber, sometimes we are able to use the methods developed by Brin in the proof of his general result mentioned in the Introduction. This is due to the existence of the pair of stable/unstable foliations for the extension and the fact that generic accessibility for the pair is easy to prove in some cases. The difficult part is to show the density of recurrent points. The compact factors in $\Gamma$ are sometimes easy to accommodate, due to the existence of compact elements. See [46] for the case of $\Gamma=S E(2)$ and $\Gamma=K \times \mathbb{R}^{n}$ with $K$ compact. 
We denote by $W^{s}(x)$ and $W^{u}(x)$ the stable and unstable leaves of the hyperbolic dynamical system $f$ through the point $x \in X$. The next lemma is a consequence of [20, Appendix A]. Detailed proofs can be found in [53].

Lemma 1. Assume that $X$ is a hyperbolic basic set for $f: X \rightarrow X$, that $\Gamma$ is a connected Lie group and $\beta: X \rightarrow \Gamma$ an $\alpha$-Hölder cocycle that has subexponential growth. Then, the $\Gamma$-extension $f_{\beta}: X \times \Gamma \rightarrow X \times \Gamma$ admits stable and unstable foliations, which are $\alpha$-Hölder and invariant under right multiplication by elements of $\Gamma$. The stable and unstable leaves of $f_{\beta}$ through $\left(x, e_{\Gamma}\right) \in X \times \Gamma$ are the graphs of the functions:

$$
\begin{aligned}
& \gamma_{x}^{s}: W^{s}(x) \rightarrow \Gamma, \quad \gamma_{x}^{s}(y)=\lim _{n \rightarrow \infty} \beta(n, x) \beta(n, y)^{-1} \\
& \gamma_{x}^{u}: W^{u}(x) \rightarrow \Gamma, \quad \gamma_{x}^{u}(y)=\lim _{n \rightarrow \infty} \beta(-n, x) \beta(-n, y)^{-1}
\end{aligned}
$$

These functions are $\alpha$-Hölder and vary continuously with the cocycle $\beta$ in the following sense: if $\beta_{k} \rightarrow \beta$ in the $C^{0}$-topology and $\beta_{k}$ remains $C^{\alpha}$-bounded, then $\gamma_{k, x}^{s} \rightarrow \gamma_{x}^{s}$ and $\gamma_{k, x}^{u} \rightarrow \gamma_{x}^{u}$ on $W_{l o c}^{s}(x)$ in the $C^{0}$-topology.

We call the values of the functions $\gamma_{x}^{s}, \gamma_{x}^{u}$ holonomies along stable/ unstable leaves.

A new criterion for topological transitivity applicable to extensions was developed in [33]. One of the key notions introduced in [33] is:

Definition 11. Let $\Gamma$ be a connected Lie group, $X$ a basic hyperbolic set for $f: X \rightarrow X$, $\beta: X \rightarrow \Gamma$ a cocycle and $f_{\beta}: X \times \Gamma \rightarrow X \times \Gamma$ the skew-extension. Given $x \in X$, let:

$$
\mathcal{L}_{\beta}(x)=\left\{\gamma \in \Gamma: \text { there exist } x_{k} \in X \text { and } n_{k}>0 \text { such that } x_{k} \rightarrow x \text { and } f_{\beta}^{n_{k}}\left(x_{k}, e_{\Gamma}\right) \rightarrow(x, \gamma)\right\}
$$

We will refer to $\mathcal{L}_{\beta}(x)$ as the range of $\beta$ over $x$.

That is, the set $\mathcal{L}_{\beta}(x)$ consists of the possible limits $\lim _{k \rightarrow \infty} \beta\left(n_{k}, x_{k}\right)$, subject to $x_{k} \rightarrow x$ and $f^{n_{k}}\left(x_{k}\right) \rightarrow x$. Note that we do not require that $n_{k} \rightarrow \infty$ or that $x_{k} \neq x$. Clearly, $\mathcal{L}_{\beta}(x)$ is a closed subset of $\Gamma$.

The following theorem is [33, Lemma 3.1, Theorem 3.3].

Theorem 11. Assume that $X$ is a hyperbolic basic set for $f: X \rightarrow X$, that $\Gamma$ is a connected Lie group and $\beta: X \rightarrow \Gamma$ a Hölder cocycle that has subexponential growth. Then:

(1) $\mathcal{L}_{\beta}(x)$ is a closed semigroup of $\Gamma$ for each $x \in X$.

(2) If there exists a point $x_{0} \in X$, such that $\mathcal{L}_{\beta}\left(x_{0}\right)=\Gamma$, then $\beta$ is a transitive cocycle. 
The following lemma is [33, Lemma 2.2]. Briefly, it says that the heights of $f_{\beta}$ over two nearby trajectories in the base are close, independent of the lengths of the trajectories. This result is used in the constructions of elements in $\mathcal{L}_{\beta}$.

Lemma 2. Assume that $X$ is a hyperbolic basic set for $f: X \rightarrow X$, that $\Gamma$ is a connected Lie group and $\beta: X \rightarrow \Gamma$ an $\alpha$-Hölder cocycle that has subexponential growth. Then, there is a constant $C_{5}>0$ with the following property.

For any $\epsilon>0$ sufficiently small, any $n \geq 1$ and any two trajectories $x_{k}=f^{k}\left(x_{0}\right)$, $y_{k}=f^{k}\left(y_{0}\right)$, such that $d_{M}\left(x_{k}, y_{k}\right)<\epsilon$ for $0 \leq k \leq n-1$,

$$
d\left(\beta\left(n, x_{0}\right), \beta\left(n, y_{0}\right)\right) \leq C_{5}\left(\left\|A d\left(\beta\left(n, x_{0}\right)\right)\right\|+1\right) \epsilon^{\alpha}
$$

\section{Admissible Sequences of Products of Holonomies}

We observe that a priori, the set $\mathcal{L}_{\beta}$ introduced in the previous section may be empty. In this section, we describe a method for obtaining elements in $\mathcal{L}_{\beta}$. We follow closely [47].

Throughout this section, $\left(M, d_{M}\right)$ is a Riemannian manifold, $X \subset M$ is a basic hyperbolic set for $f: X \rightarrow X$ with contraction constant $\lambda \in(0,1)$ satisfying (1), $\Gamma$ a connected Lie group and $\beta: X \rightarrow \Gamma$ an $\alpha$-Hölder cocycle that has subexponential growth.

Definition 12. By a periodic heteroclinic cycle, we mean a cycle consisting of points $p_{1}, \ldots, p_{k}$ that are periodic for the map $f$, have disjoint trajectories, such that $p_{j}$ is transverse heteroclinic to $p_{j+1}$ through a point $\zeta_{j} \in W^{u}\left(p_{j}\right) \cap W^{s}\left(p_{j+1}\right)$, for $j=1, \ldots, k$ (where $\left.p_{k+1}=p_{1}\right)$.

Let $P_{1}, \ldots, P_{k}$ be the corresponding periodic orbits and denote the periods by $\ell_{1}, \ldots, \ell_{k}$. Denote by $O_{j}$ the heteroclinic trajectory from $p_{j}$ to $p_{j+1}$ (of the point $\zeta_{j}$ chosen above), and by $H_{j}$ the holonomy along this heteroclinic connection (that is, along $W^{u}\left(p_{j}\right)$ from $p_{j}$ to $\zeta_{j}$ and then along $W^{s}\left(p_{j+1}\right)$ from $\zeta_{j}$ to $\left.p_{j+1}\right)$.

Replace the heteroclinic orbit $O_{j}$ from $p_{j}$ to $p_{j+1}$ by the trajectory $Q_{j}$ of length $\ell_{j} M_{j}+\ell_{j+1} M_{j+1}$ that spends time $\ell_{j} M_{j}$ in the first half of $O_{j}$ and time $\ell_{j+1} M_{j+1}$ in the second half of $O_{j}$; that is, $Q_{j}=\left\{f^{n}\left(\zeta_{j}\right) \mid-\ell_{j} M_{j} \leq n<0\right\} \cup\left\{f^{n}\left(\zeta_{j}\right) \mid 0 \leq n<\right.$ $\left.\ell_{j+1} M_{j+1}\right\}$ ). For the trajectory connecting $p_{k}$ to $p_{k+1}$, we allow $M_{1}$ and $M_{k+1}$ to be distinct. The positive integers $M_{j}$ will be chosen later.

Consider the heights $\beta\left(P_{j}\right)$ and $\beta\left(Q_{j}\right)$ over the periodic orbits $P_{j}$ and trajectories $Q_{j}$. 
Lemma 3. For $j=1, \ldots, k$, the limit:

$$
\lim _{M_{j}, M_{j+1} \rightarrow \infty} \beta\left(P_{j}\right)^{-M_{j}} \beta\left(Q_{j}\right) \beta\left(P_{j+1}\right)^{-M_{j+1}}=H_{j}
$$

exists and is the product of the holonomies along the unstable and stable leaves of $\mathrm{O}_{j}$, from $p_{j}$ to $p_{j+1}$.

Proof. This follows from Lemma 1.

Definition 13. Consider a sequence of vectors $N(1), N(2) \ldots \in \mathbb{N}^{k+1}$ whose entries are positive integers. Write $N(i)=\left(M_{1}(i), \ldots, M_{k+1}(i)\right)$. The sequence is admissible if there is a constant $C_{2} \geq 1$, such that $M_{p}(i) / M_{q}(i) \leq C_{2}$, for all $p, q=1 \ldots, k+1$ and all $i \geq 1$.

If $N=\left(M_{1}, \ldots, M_{k+1}\right)$ is a sequence, we write $N \rightarrow \infty$ if $M_{p} \rightarrow \infty$ for $p=1, \ldots, k+1$.

Theorem 12. Let $N=\left(M_{1}, \ldots, M_{k+1}\right) \in \mathbb{N}^{k+1}$. Define:

$$
A(N)=\beta\left(P_{1}\right)^{M_{1}} H_{1} \beta\left(P_{2}\right)^{2 M_{2}} H_{2} \cdots \beta\left(P_{k}\right)^{2 M_{k}} H_{k} \beta\left(P_{1}\right)^{M_{k+1}}
$$

If the limit $A=\lim _{N \rightarrow \infty} A(N)$ exists along an admissible sequence $N(1), N(2), \ldots$, then $A \in \mathcal{L}_{\beta}\left(p_{1}\right)$.

In the remainder of this section, we prove Theorem 12. From now on, we assume for notational simplicity that $P_{j}=p_{j}$ are fixed points (so, $\ell_{j}=1$ ).

Given $N=\left(M_{1}, \ldots, M_{k+1}\right) \in \mathbb{N}^{k+1}$, define:

$|N|=\left(M_{1}+M_{k+1}\right) / 2+\sum_{j=2}^{k} M_{j}, \min N=\min \left\{M_{1}, \ldots, M_{k+1}\right\}, \max N=\max \left\{M_{1}, \ldots, M_{k+1}\right\}$

Note that for an admissible sequence $N$, we have $\max N \leq C_{2} \min N$. Define:

$$
H_{j}(N)=\beta\left(P_{j}\right)^{-M_{j}} \beta\left(Q_{j}\right) \beta\left(P_{j+1}\right)^{-M_{j+1}} .
$$

By Lemma $3, \lim _{N \rightarrow \infty} H_{j}(N)=H_{j}$ (independent of the sequence $N$ ). Moreover, by [20, proof of Theorem $4.3(\mathrm{~g})]$, there is $\delta_{0} \in(0,1)$, such that:

$$
d\left(H_{j}(N), H_{j}\right)=O\left(\delta_{0}^{\min N}\right)
$$

Recall that $Q_{j}$ is a trajectory of length $M_{j}+M_{j+1}$ that shadows the heteroclinic connection from $p_{j}$ to $p_{j+1}$. Concatenate these trajectories to form a periodic pseudo-orbit $Q=Q_{1} \ldots Q_{k}$ of length $2|N|$. Then, $Q$ is a $\delta$-pseudo-orbit with $\delta \leq C_{3} \lambda^{\min N}$, where $C_{3}>0$ is a constant (depending on $f: X \rightarrow X$ ) and $\lambda$ is 
the contraction constant. By the hyperbolicity of $X$, there is a periodic orbit $\tilde{Q}$ of length $2|N|$ that $\epsilon$-shadows $Q$ with $\epsilon \leq C_{4} \lambda^{\min N}$, where $C_{4}>0$ is a constant. See [54, page 74] for standard shadowing techniques.

\section{Proposition 1.}

(1) $\beta(Q)=\beta\left(P_{1}\right)^{M_{1}} H_{1}(N) \beta\left(P_{2}\right)^{2 M_{2}} H_{2}(N) \cdots \beta\left(P_{k}\right)^{2 M_{k}} H_{k}(N) \beta\left(P_{1}\right)^{M_{k+1}}$.

(2) $\lim _{N \rightarrow \infty} d(\beta(Q), \beta(\tilde{Q}))=0$ along admissible sequences $N$.

(3) $\lim _{N \rightarrow \infty} d(\beta(Q), A(N))=0$ along admissible sequences $N$.

Proof. Part (1) is a direct calculation, namely:

$$
\beta(Q)=\prod_{j=1}^{k} \beta\left(Q_{j}\right)=\prod_{j=1}^{k} \beta\left(P_{j}\right)^{M_{j}} H_{j}(N) \beta\left(P_{j+1}\right)^{M_{j+1}}
$$

Next, write $\tilde{Q}=\tilde{Q}_{1} \ldots \tilde{Q}_{k}$ where $\tilde{Q}_{j}$ has length $M_{j}+M_{j+1}$. Define $\gamma_{j}=\beta\left(Q_{j}\right)$, $\tilde{\gamma}_{j}=\beta\left(\tilde{Q}_{j}\right)$. Note that $Q_{j}$ and $\tilde{Q}_{j}$ have a length of at most $2 \max N$ and that $\tilde{Q}_{j} \epsilon$-shadows $Q_{j}$ with $\epsilon \leq C_{4} \lambda^{\min N}$. It follows from Lemma 2 that $d\left(\gamma_{i}, \tilde{\gamma}_{i}\right) \leq$ $C \lambda^{\alpha \min N}\left(\left\|\operatorname{Ad}\left(\gamma_{i}\right)\right\|+1\right)$ where $C=C_{4}^{\alpha} C_{5}$. Hence, using the properties of the metric on $\Gamma$ and the fact that $\beta$ has subexponential growth, we have:

$$
\begin{aligned}
d(\beta(Q), \beta(\tilde{Q}))= & d\left(\gamma_{1} \gamma_{2} \cdots \gamma_{k}, \tilde{\gamma}_{1} \tilde{\gamma}_{2} \cdots \tilde{\gamma}_{k}\right) \\
\leq & d\left(\gamma_{1} \gamma_{2} \cdots \gamma_{k}, \tilde{\gamma}_{1} \gamma_{2} \cdots \gamma_{k}\right)+d\left(\tilde{\gamma}_{1} \gamma_{2} \gamma_{3} \cdots \gamma_{k}, \tilde{\gamma}_{1} \tilde{\gamma}_{2} \gamma_{3} \cdots \gamma_{k}\right)+ \\
& \quad \cdots+d\left(\tilde{\gamma}_{1} \tilde{\gamma}_{2} \cdots \tilde{\gamma}_{k-1} \gamma_{k}, \tilde{\gamma}_{1} \tilde{\gamma}_{2} \cdots \tilde{\gamma}_{k-1} \tilde{\gamma}_{k}\right) \\
\leq & d\left(\gamma_{1}, \tilde{\gamma}_{1}\right)\left\|\operatorname{Ad}\left(\gamma_{2} \cdots \gamma_{k}\right)\right\|+d\left(\gamma_{2}, \tilde{\gamma}_{2}\right)\left\|\operatorname{Ad}\left(\gamma_{3} \cdots \gamma_{k}\right)\right\|+\cdots+d\left(\gamma_{k}, \tilde{\gamma}_{k}\right) \\
\leq & C \lambda^{\alpha \min N}\left[(1+\eta)^{2 \max N}+1\right]\left[(1+\eta)^{2 \max N}+\cdots+(1+\eta)^{2(k-1) \max N}\right]
\end{aligned}
$$

where $\eta>0$ can be chosen arbitrarily small and $\|\operatorname{Ad}(\beta(n, x))\| \leq(1+\eta)^{n}$ for $n$ large enough. Restricting to admissible sequences, $\min N$ and $\max N$ are comparable, and Part (2) follows. The proof of Part (3) is similar using (5).

Proof of Theorem 12. By assumption, $A(N) \rightarrow A$. Hence, by Proposition 1, Part $(2,3), \beta(\tilde{Q}) \rightarrow A$. We conclude that $A \in \mathcal{L}_{\beta}\left(p_{1}\right)$ by definition of $\mathcal{L}_{\beta}\left(p_{1}\right)$.

Remark 7. Proving that the limit $A=\lim _{N \rightarrow \infty} A(N)$ exists is challenging. The proof depends on the group that appears in the fiber. If $\Gamma$ is the Heisenberg group, the components of $A(N)$ are a quadratic and several linear polynomials. In order to extract a convergent subsequence, we need to show that a diophantine system of a quadratic and several linear equations has approximate solutions. A difficulty is that, even though we can prescribe the leading coefficients in these equations, we cannot control all of the coefficients. Lower 
order coefficients depend on the holonomy factors in $A(N)$, which are difficult to control generically. This is the place where the diophantine approximation results, such as Theorem 7 , come into play.

\section{Semigroup Problem}

For many Lie groups $\Gamma$, it is not hard to show that, for an integer $p>0$ big enough, there is a large open set $U \subset \Gamma^{p}$, such that if $F \in U$, then the family $F$ generates $\Gamma$, that is the group generated by $F$ is dense in $\Gamma$. The proof of transitivity of an extension $f_{\beta}$ is based on showing that the set $\mathcal{L}_{\beta}(x)$ of "heights" of $\beta$ over a (periodic) point $x$ is the whole fiber $\Gamma$. See Theorem 11. To obtain the condition $\mathcal{L}_{\beta}(x)=\Gamma$, we have to prove that for a typical family $F \in \Gamma^{p}$ that generates $\Gamma$ as a group, if $F$ is not contained in a maximal sub-semigroup with a non-empty interior, then $F$ generates $\Gamma$ as a semigroup, as well. We refer to this question as the semigroup problem. For example, if $\Gamma=\mathbb{R}$, the problem states that if a set $S$ contains both positive and negative numbers, then the closure of the semigroup generated by $S$ is a group.

The semigroup problem was solved for $\Gamma=\mathbb{R}^{n}[41]$ and more generally for groups of the form $\Gamma=K \times \mathbb{R}^{n}$, where $K$ is a compact Lie group [33, Theorem 5.10]. It is also solved for $\Gamma=S E(n)$ [33, Theorem 6.8], for certain solvable groups that are semidirect products of $\mathbb{R}^{n}$ with $\mathbb{R}^{m}$, such as $A f f{ }^{+}$in [55], and for the Heisenberg group in [48].

Acknowledgments: Viorel Nitica was partially supported by the Simons Foundation Grant 208729. Andrew Török was partially supported by the Simons Foundation Grant 239583.

Conflicts of Interest: The authors declare no conflict of interest.

\section{References}

1. Blanchard, F. Topological chaos: What may this mean? J. Differ. Equ. Appl. 2009, 15, 23-46.

2. Kolyada, S.; Snoha, L. Some aspects of topological transitivity-A survey. Grazer Math. Ber. 1997, 334, 3-35.

3. Devaney, R.L. An Introduction to Chaotic Dynamical Systems, 2nd ed.; Addison-Wesley: Redwood City, CA, USA, 1989.

4. Banks, J.; Brooks, J.; Cairns, J.; Davis, G.; Stacey, P. On Devaney's definition of chaos. Am. Math. Monthly 1992, 99, 332-334.

5. Oxtoby, J.; Ulam, S. Measure-preserving homeomorphisms and metrical transitivity. Ann. Math. 1941, 42, 874-920.

6. Besicovitch, A.S. A problem on topological transformation of the plane. Fund. Math. 1937, 28, 61-65.

7. Besicovitch, A.S. A problem on topological transformation of the plane II. Proc. Cambr. Phil. Soc. 1951, 47, 38-45. 
8. Shnirelman, L.G. An example of a transformation of the plane. Izv. Donsk. Polytech. Inst. 1930, 14, 64-74. (In Russian)

9. Sidorov, Ye. A. Topologically transitive cylindrical cascades. Mat. Zametki 1973, 14, 441-452. (In Russian)

10. Anosov, D. V.; Sinai, Ja.G. Certain smooth ergodic systems. Uspehi Mat. Nauk 1967, 22, 107-172. (In Russian)

11. Bowen, R. Equilibrium States and the Ergodic Theory of Anosov Diffeomorphisms; Lecture Notes in Mathematics; Springer-Verlag: Berlin, Germany, 1975; Volume 470.

12. Smale, S. Differentiable dynamical systems. Bull. Amer. Math. Soc. 1967, 73, 747-817.

13. Katok, A.; Hasselblatt, B. Introduction to the Modern Theory of Dynamical Systems; Cambridge University Press: Cambridge , UK, 1995.

14. Hopf, E. Statistik der geodätischen Linien in Mannigfaltigkeiten negativer Krümmung. Ber. Verh. Sachs. Akad. Wiss. Leipzig 1939, 91, 261-304. (In German)

15. Anosov, D.V. Geodesic Flows on Closed Riemannian Manifolds with Negative Curvature. Trudy Mat. Inst. Steklov 1967, 90, 209. (In Russian)

16. Brin, M.; Pesin, Y. Partially hyperbolic dynamical systems. Izvestia Akad. Nauk SSSR Ser. Mat. 1974, 38, 170-212. (In Russian)

17. Grayson, M.; Pugh, C.; Shub, M. Stably ergodic diffeomorphisms. Ann. Math. 1994, 140, 295-329.

18. Pugh, C.; Shub, M. Stable ergodicity and julienne quasi-conformality. J. Eur. Math. Soc. 2000, 2, 1-52.

19. Burns, K.; Wilkinson, A. On the ergodicity of partially hyperbolic systems. Ann. Math. 2010, 171, 451-489.

20. Nitica, V.; Török, A. An open and dense set of stably ergodic diffeomorphisms in a neighborhood of certain nonergodic ones. Topology 2001, 40, 259-278.

21. Hertz, F. R. Stable ergodicity of certain linear automorphisms of the torus. Ann. Math. 2005, 162, 65-107.

22. Dolgopyat, D; Wilkinson, A. Stable accessibility is $C^{1}$ dense. In Geometric Methods in Dynamics II, 2003; Volume 287, pp. 33-60.

23. Brin, I.M. Topological transitivity of a class of dynamical systems and frame flow on manifolds of negative curvature. Funct. Anal. Appl. 1975, 9, 9-19.

24. Bonatti, C.; Díaz, L.J. Persistent nonhyperbolic transitive diffeomorphisms. Ann. Math. 1996, 143, 357-396.

25. Shub, M. Topological transitive diffeomorphism on $T^{4}$. Lect. Notes in Math. 1971, 206, 39-40.

26. Bonatti, C.; Crovisier, S. Récurrence et généricité. Invent. Math. 2004, 158, 33-104. (In French)

27. Pugh, C. The closing lemma. Amer. J. Math. 1967, 89, 956-1009.

28. Hayashi, S. Connecting invariant manifolds and the solution of the $C^{1}$-stability and Q-stability conjectures for flows. Ann. Math. 1997, 145, 81-137.

29. Hayashi, S. Corrections to: "Connecting invariant manifolds and the solution of the $C^{1}$-stability and $Q$-stability conjectures for flows". Ann. Math. 1999, 150, 353-356. 
30. Grosse-Erdmann, K.-G.; Peris, A. Linear Chaos; Springer: Berlin, Germany, 2011.

31. Pollicott, M.; Walkden, C.P. Livšic theorems for connected Lie groups. Trans. Amer. Math. Soc. 2001, 353, 2879-2895.

32. Dungey, N.; ter Elst, A.F.M.; Robinson, D.W. Analysis on Lie Groups with Polynomial Growth; Progress in Mathematics; Birkhauser Boston Inc.: Boston, MA, USA, 2003.

33. Melbourne, I.; Nitica, V.; Török, A. Stable transitivity of certain noncompact extensions of hyperbolic systems. Ann. Henri Poincare 2005, 6, 725-746.

34. Nitica, V. A note about topologically transitive cylindrical cascades. Isr. J. Math. 2001, 126, 141-156.

35. Nitica, V. Examples of topologically transitive skew-products. Discret. Contin. Dyn. Syst. 2000, 6, 351-360.

36. Field, M.; Melbourne, I.; Török, A. Stable ergodicity for smooth compact Lie group extensions of hyperbolic basic sets. Ergodic Theory Dyn. Syst. 2005, 25, 517-551.

37. Field, M.; Nitica, V. Stable topological transitivity of skew and principal extensions. Nonlinearity 2001, 14, 1055-1069.

38. Field, M.; Parry, W. Stable ergodicity of skew extensions by compact Lie groups. Topology 1999, 167-187.

39. Parry, W.; Pollicott, M. Stability of mixing for toral extensions of hyperbolic systems. Proc. Steklov Inst. Math. 1990, 216, 350-359.

40. Burns, K.; Wilkinson, A. Stable ergodicity of skew products. Ann. Sci. Ecole Norm. Sup. 1999, 32, 859-889.

41. Nitica, V.; Pollicott, M. Transitivity of Euclidean extensions of Anosov diffeomorphisms. Ergod. Theory Dyn. Syst. 2005, 25, 257-269.

42. Bousch, T. La condition de Walter. Ann. Sci. Ecole Norm. Sup. 2001, 34, 287-311. (In French)

43. Bowen, R. On Axiom A Diffeomorphisms; American Mathematical Society: Providence, RI, USA, 1977; Volume 35.

44. Moss, A.; Walkden, C.P. Stable topological transitivity properties of $\mathbb{R}^{n}$-extensions of hyperbolic transformations. Ergod. Theory Dyn. Syst. 2012, 32, 1435-1443.

45. Melbourne, I.; Nicol, M. Stable transitivity of Euclidean group extensions. Ergod. Theory Dyn. Syst. 2003, 23, 611-619.

46. Melbourne, I.; Nitica, V.; Török, A. A note about stable transitivity of noncompact extensions of hyperbolic systems. Discret. Contin. Dyn. Syst. 2006, 14, 355-363.

47. Melbourne, I.; Nitica, V.; Török, A. Transitivity of Euclidean-type extensions of hyperbolic systems. Ergod. Theory Dyn. Syst. 2009, 29, 1585-1602.

48. Nitica, V.; Török, A. Stable transitivity of Heisenberg group extensions of hyperbolic systems. Nonlinearity 2014, 27, 661-683.

49. Melbourne, I.; Nitica, V.; Török, A. Transitivity of Heisenberg group extensions of hyperbolic systems. Ergod. Theory Dyn. Syst. 2012, 32, 223-235.

50. Nitica, V. Stably transitivity for extensions of hyperbolic systems by semidirect products of compact and nilpotent Lie groups. Discret. Contin. Dyn. Syst. 2011, 29, 1197-1203.

51. Djoković, D.Z. The union of compact subgroups of a connected locally compact group. Math. Z. 1978, 158, 99-105. 
52. Kuranishi, M. On everywhere dense imbeddings of free groups in Lie groups. Nagoya Math. J. 1951, 2, 63-71.

53. Katok, A.; Nitica, V. Rigidity of Abelian Group Actions. I, Introduction to Cocycle Problem; Cambridge University Press: Cambridge , UK, 2011.

54. Newhouse, S. Lectures on Dynamical Systems. In Dynamical Systems; Progress in Mathematics; Springer: Berlin, Germany, 1980; pp. 1-114.

55. Lui, K.; Nitica, V.; Venkatesh, S. The semigroup problem for central semidirect product of $\mathbb{R}^{n}$ with $\mathbb{R}^{m}$. Topol. Proc. 2015, 45, 9-29. 

MDPI AG

St. Alban-Anlage 66

4052 Basel, Switzerland

Tel. +41616837734

Fax +41 613028918

http://www.mdpi.com

Axioms Editorial Office

E-mail: axioms@mdpi.com

http://www.mdpi.com/journal/axioms

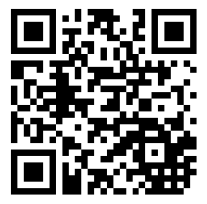



MDPI AG

St. Alban-Anlage 66

4052 Basel

Switzerland

Tel: +41 616837734

Fax: +41 613028918 